\title{
Essays on Information, \\ Coordination, and Monetary Policy
}

\author{
Anne Paess, MSc
}

Submitted to Swansea University in fulfilment of the requirements for the Degree of

Doctor of Philosophy

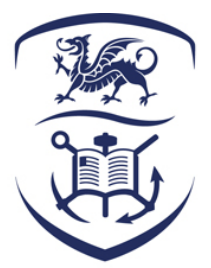

Swansea University

Prifysgol Abertawe

Swansea University

2019 


\section{Abstract}

Greater transparency in policymaking has been a distinct trend among the world's major central banks in the past 30 years. This trend reflects an established view in the central-banking community that managing expectations in the economy through communication is an important element of monetary policy. This view implies that there is an inherent connection between central bank transparency, private sector knowledge, and effectiveness of policy.

The aim of this thesis is to add to the theoretical body of work by considering optimal policy design in the context of a narrow set of game-theoretic models with strategic complementarities and heterogeneity in private sector information. Each model variation is defined in terms of assumptions about information quality, its acquisition and dissemination. We evaluate policy performance in terms of social welfare outcomes as well as its ability to eliminate informational inefficiencies.

In Chapter 2 we extend the seminal Morris and Shin (2002) model to include information stickiness in the private sector, central bank transparency, and direct policy intervention. We find that in our model (i) commitment performs at least as well as discretionary policy, and (ii) optimally designed policy fails to achieve an efficient outcome.

In Chapter 3 we extend the Morris and Shin (2002) model to include costly private information acquisition, central bank transparency, and direct policy intervention. In an economy with a fixed information cost, the combination of full opacity and an optimally designed policy rule achieves efficiency; whereas, in an economy with a linear-cost structure, optimal policy either eliminates or at least mitigates informational inefficiencies.

In Chapter 4 we extend the Morris and Shin (2018) market signal model to include costly information acquisition. We consider private and public information acquisition. We look at how information value of private sector actions enters the central banks optimal commitment strategies. 


\title{
Declaration and Statements
}

\author{
DECLARATION
}

This work has not previously been accepted in substance for any degree and is not being concurrently submitted in candidature for any degree.

Signed ............................................................ (candidate)

Date

\section{STATEMENT 1}

This thesis is the result of my own investigations, except where otherwise stated. Other sources are acknowledged by footnotes giving explicit references. A bibliography is appended.

Signed (candidate)

Date

\section{STATEMENT 2}

I hereby give consent for my thesis, if accepted, to be available for photocopying and for inter-library loan, and for the title and summary to be made available to outside organisations.

Signed (candidate)

Date 


\section{Contents}

Acknowledgements $\quad$ x

List of Tables $\quad$ xi

List of Figures xiii

List of Abbreviations $\quad$ Xv

$\begin{array}{ll}\text { Introduction } & 1\end{array}$

1 Literature Review 3

1.1 Introduction . . . . . . . . . . . . . . . . . 3

1.2 Central bank transparency: practical considerations and their implications for theory . . . . . . . . . . . . . . . 4

1.2.1 Desirability and feasibility of transparency . . . . . . . . 5

1.2.2 Implementing various degrees of transparency . . . . . . . . 7

1.2.3 Transparency as a policy tool . . . . . . . . . . . . . . 9

1.3 Monetary policy regimes: commitment versus discretion . . . . . . . 13

1.4 Social value of information and coordination . . . . . . . . . . . 16

1.4.1 Coordination and the Morris and Shin framework . . . . . . . 18

1.4.2 Extensions of the Morris and Shin framework . . . . . . . . . 21

1.5 Selected topics in information . . . . . . . . . . . . 26

1.5.1 Sticky information . . . . . . . . . . . . . 26

1.5.2 Costly information acquisition . . . . . . . . . . . . 28

1.5.3 Signal value of market aggregates . . . . . . . . . . . . . 30

1.6 Concluding remarks . . . . . . . . . . . . . . . . . . . . . 34

2 Sticky Information $\quad 35$

2.1 Introduction . . . . . . . . . . . . . . . . . . . . . 35

2.2 Model . . . . . . . . . . . . . . . . . . . . . . . 37

2.2 .1 Private Sector . . . . . . . . . . . . . . . 37

2.2.1.1 Coordination in equilibrium, and efficiency . . . . . 38 
2.2.1.2 Hybrid payoff function . . . . . . . . . . . . . 42

2.2.1.3 Passive agents . . . . . . . . . . . . . . . . . 48

2.2.1.4 Active agents . . . . . . . . . . . . . 49

2.2.1.5 Population action profile . . . . . . . . . 50

2.2.1.6 Social welfare . . . . . . . . . . . . . . . 51

2.2.2 Central Bank . . . . . . . . . . . . . . . . . . . 51

2.2.2.1 Central bank communication . . . . . . . . 51

2.2.2.2 Direct policy intervention . . . . . . . . . . 52

2.2.3 Private sector expectations . . . . . . . . . . . . . 53

2.3 Commitment to a Rule . . . . . . . . . . . . . . . . . . . . . 55

2.3.1 Equilibrium without passive agents $(Q=0) \quad \ldots \ldots 55$

2.3.2 Equilibrium with passive agents $(0<Q<1)$. . . . . . . 57

2.3.2.1 Hybrid payoff function $(0<\lambda<1)$. . . . . . . . 59

2.3.2.2 M-S payoff function $(\lambda=0) \ldots \ldots$. . . . . 60

2.3.2.3 J-L payoff function $(\lambda=1) \ldots \ldots$. . . . . . 62

2.4 Discretion . . . . . . . . . . . . . . . . . . . . 63

2.4.1 Equilibrium without passive agents $(Q=0) \ldots \ldots 4$

2.4.2 Equilibrium with passive agents $(0<Q<1) \ldots . . . . .66$

2.4.2.1 Hybrid payoff function $(0<\lambda<1) \ldots \ldots 8$

2.4.2.2 M-S payoff function $(\lambda=0) \ldots \ldots . \ldots 70$

2.4.2.3 J-L payoff function $(\lambda=1) \ldots \ldots . . . \ldots 71$

2.4.3 Equilibrium without passive agents re-attempted $(Q \rightarrow 0) \quad$. 72

2.4.4 Equilibrium without active agents $(Q=1) \ldots 73$

2.5 Optimal Monetary Policy Regime . . . . . . . . . . . . . . . . . . 77

2.5.1 M-S payoff function $(\lambda=0) \ldots \ldots$. . . . . . 80

2.5.2 J-L payoff function $(\lambda=1) \ldots \ldots . \ldots . \ldots . . \ldots 80$

2.6 Efficient Benchmark . . . . . . . . . . . . . . . . . 81

2.6.1 Efficient case without passive agents $(Q=0) \ldots . . . .82$

2.6.2 Efficient case with passive agents $(0<Q<1) \ldots . . . . .84$

2.6.3 Efficiency of equilibria with optimal policy . . . . . . . . 86

2.7 Summary table of Chapter 2 results . . . . . . . . . . . . . . . . . 90

2.8 Concluding remarks . . . . . . . . . . . . . . . . . . . . . . . 93

3 Costly Information $\quad 96$

3.1 Introduction . . . . . . . . . . . . . . . . 96

3.2 Model . . . . . . . . . . . . . . . . . . . . . . . . . 98

3.2 .1 Private Sector . . . . . . . . . . . . . . . . . . . . 98

3.2.1.1 Payoff functions and coordination . . . . . . . . . 98

3.2.1.2 Information acquisition . . . . . . . . . . 100 
3.2 .2 Central Bank . . . . . . . . . . . . . . . . . . . . . . 101

3.2.2.1 Central bank transparency (CBT) . . . . . . . 101

3.2.2.2 Direct policy intervention . . . . . . . . . . . . 102

3.2 .3 Game form . . . . . . . . . . . . . . . . . . . . . . 103

3.2 .4 Information use . . . . . . . . . . . . . . . . . . . 104

3.2.4.1 Private sector expectations . . . . . . . . . . 105

3.2.4.2 Purchasing agents . . . . . . . . . . . . 105

3.2.4.3 Non-purchasing agents . . . . . . . . . . . . 107

3.2 .5 Social welfare . . . . . . . . . . . . . . . . . 108

3.2.5.1 Expected social welfare . . . . . . . . . . . . 108

3.2.5.2 Policy and CBT in N-NE . . . . . . . . . . 110

3.3 Fixed Cost: Morris-Shin Payoff Function . . . . . . . . . . . . . 112

3.3 .1 In the absence of policy . . . . . . . . . . . . . . . 112

3.3.1.1 Purchasing equilibria . . . . . . . . . . . . . . . 112

3.3.1.2 Welfare and CBT in P-NE . . . . . . . . . . . 115

3.3.1.3 Non-purchasing equilibria . . . . . . . . . . . . 118

3.3.1.4 Equilibrium conditions for P-NE and N-NE . . . . 120

3.3.1.5 Efficient benchmark . . . . . . . . . . . . . . 124

3.3.1.6 Efficiency of equilibria . . . . . . . . . . . 126

3.3.2 With direct policy intervention . . . . . . . . . . . 128

3.3.2.1 Purchasing equilibria . . . . . . . . . . . . . . . . 128

3.3.2.2 Welfare, instrument adjustment, and CBT in P-NE . 129

3.3.2.3 Non-purchasing equilibria . . . . . . . . . . . . . 131

3.3.2.4 Equilibrium conditions for P-NE and N-NE . . . . 134

3.3.2.5 Efficiency of equilibria . . . . . . . . . . . 136

3.3.3 Effect of policy on information acquisition . . . . . . . . 137

3.4 Fixed Cost: James-Lawler Payoff Function . . . . . . . . . . . 138

3.4 .1 In the absence of policy $\ldots \ldots \ldots \ldots$

3.4.1.1 Purchasing equilibria . . . . . . . . . . . . . . . 138

3.4.1.2 Non-purchasing equilibria . . . . . . . . . . . . 139

3.4.1.3 Equilibrium conditions for P-NE and N-NE . . . . 140

3.4.1.4 Efficient benchmark . . . . . . . . . . . . . . . 141

3.4.1.5 Efficiency of equilibria . . . . . . . . . . . . . 142

3.4.2 With direct policy intervention . . . . . . . . . . . . . 144

3.4.2.1 Purchasing equilibria . . . . . . . . . . . . . . . 144

3.4.2.2 Welfare, instrument adjustment, and CBT in P-NE . 145

3.4.2.3 Non-purchasing equilibria . . . . . . . . . . . . 146

3.4.2.4 Equilibrium conditions for P-NE and N-NE . . . . 147

3.4.2.5 Efficiency of equilibria . . . . . . . . . . . . 149 
3.4.3 Effect of policy on information acquisition . . . . . . . . 150

3.5 Linear Cost: Morris-Shin Payoff Function . . . . . . . . . . . . . . . . 151

3.5.1 In the absence of policy . . . . . . . . . . . . . 151

3.5.1.1 Private information acquisition . . . . . . . . 151

3.5.1.2 Central bank transparency . . . . . . . . . . . . . 154

3.5.1.3 Efficient benchmark . . . . . . . . . . . . 155

3.5.1.4 Efficiency of equilibria . . . . . . . . . 157

3.5.2 With direct policy intervention . . . . . . . . . . . 158

3.5.2.1 Purchasing equilibria: private information acquisition 158

3.5.2.2 Purchasing equilibria: instrument adjustment and CBT . . . . . . . . . . . . . . 160

3.5.2.3 Non-purchasing equilibria . . . . . . . . . . . . 162

3.5.2.4 Inducing a purchasing equilibrium . . . . . . . . 163

3.5.2.5 Efficiency of equilibria . . . . . . . . . . . 165

3.5.3 Effect of policy on private information acquisition . . . . . . 165

3.6 Linear Cost: James-Lawler Payoff Function . . . . . . . . . . . . . . 166

3.6.1 In the absence of policy . . . . . . . . . . . 166

3.6.1.1 Private information acquisition . . . . . . . 166

3.6.1.2 Central bank transparency . . . . . . . . . . . . 168

3.6.1.3 Efficient benchmark . . . . . . . . . . . . . . 169

3.6.1.4 Efficiency of equilibria . . . . . . . . . . 170

3.6.2 With direct policy intervention . . . . . . . . . . . 171

3.6.2.1 Purchasing equilibria: private information acquisition 171

3.6.2.2 Purchasing equilibria: instrument adjustment and CBT . . . . . . . . . . . . . . . 172

3.6.2.3 Non-purchasing equilibria . . . . . . . . . . . . 174

3.6.2.4 Inducing a purchasing equilibrium . . . . . . . . 175

3.6.2.5 Efficiency of equilibria . . . . . . . . . . 176

3.6.3 Effect of policy on private information acquisition . . . . . . 177

3.7 Concluding remarks . . . . . . . . . . . . . . . . . . . . . . 179

4 Market Signal Value and Costly Information 182

4.1 Introduction . . . . . . . . . . . . . . . . . . . . . . . . . 182

4.2 Private information acquisition . . . . . . . . . . . . . 184

4.2 .1 Model . . . . . . . . . . . . . . . . . . . . . 184

4.2.2 Benchmark for private information acquisition $(\tau=0) \quad \ldots 187$

4.2.3 Solving for purchasing equilibria (P-NE) . . . . . . . . . . . 189

4.2.3.1 Agent $i$ 's best response and CB's information quality 190

4.2.3.2 Information acquisition . . . . . . . . . . 192 
4.2.3.3 Agent $i$ 's expected equilibrium payoff in P-NE . . . . 195

4.2.3.4 Implications for policy design . . . . . . . . . . 198

4.2.3.5 CB's minimisation problem and numerical examples 201

4.2.4 Solving for non-purchasing equilibria (N-NE) . . . . . . . . 205

4.2.4.1 Agent $i$ 's expected equilibrium payoff in N-NE . . . . 207

4.2.4.2 Numerical examples of N-NE . . . . . . . . . . 209

4.3 Public information acquisition . . . . . . . . . . . . . . . 212

4.3 .1 Model variation . . . . . . . . . . . . . . . . . . . . 212

4.3.2 Solving for purchasing equilibria (P-NE) . . . . . . . . . . 214

4.3.3 Solving for non-purchasing equilibria $(\mathrm{N}-\mathrm{NE})$. . . . . . . . 218

4.3.4 Implications for policy design . . . . . . . . . . . . . 221

4.3.4.1 Cases in which only one type of NE exists . . . . . . 222

4.3.4.2 Inducing the N-NE when both NE types exist . . . . 224

4.3.4.3 Global optimality when both NE types exist . . . . . 226

4.4 Concluding remarks . . . . . . . . . . . . . . . . . . . . 232

$\begin{array}{ll}\text { Conclusion } & 235\end{array}$

$\begin{array}{lr}\text { Appendices } & 238\end{array}$

A $\quad 238$

A.1 Summary table of extensions and variations of the Morris and Shin

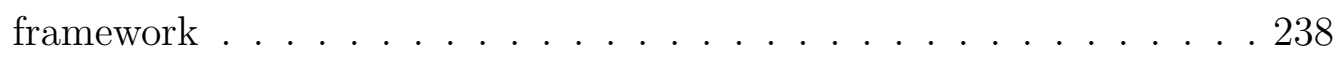

B

B.1 Hybrid payoff function: derivation of degrees of coordination . . . . . 242

B.2 Derivation of the ex ante expected equilibrium welfare . . . . . . . 245

B.3 Derivation of the expected values of $\theta$ and $z \ldots . . . . .247$

B.4 Commitment to a rule: optimal transparency for $0<\lambda<1 \ldots 249$

B.5 Commitment to a rule: examples of optimal transparency (J-L case) . 251

B.6 Discretion: shape of the expected equilibrium welfare function (hybrid case) . . . . . . . . . . . . . . . . . . . . 253

B.7 Discretion: examples of optimal transparency (J-L case) . . . . . . . 256

B.8 Example of policy performance under commitment and discretion (MS case ) . . . . . . . . . . . . . . . . . . . . . 258

B.9 Example of policy performance under commitment and discretion ( $\mathrm{J}$ -

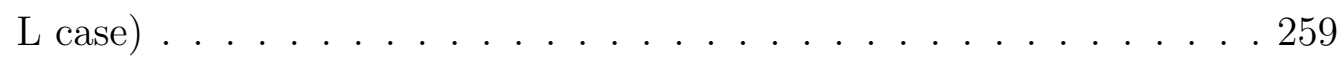

C

C.1 Model: re-formulated payoff functions . . . . . . . . . . . . . . . . . . 260

C.2 Model: deriving the degrees of coordination . . . . . . . . . . . 262 
C.3 Model: volatility and dispersion of actions . . . . . . . . . . . . 266

C.4 Fixed cost: M-S payoff function, no policy - efficiency of equilibria . . 269

C.5 Linear cost: information accuracy and commonality . . . . . . . . . 273

D 276

D.1 Agent $i$ 's forecast of instrument adjustment $g$ and its accuracy . . . . 276

D.2 Agent $i$ 's expected payoff as dispersion and volatility . . . . . . . . . 280

D.3 Characterising the solution to the CB's minimisation problem . . . . 283 


\section{Acknowledgements}

I am especially indebted to my supervisors Prof. Phillip Lawler and Dr Jonathan James for their invaluable research support and academic advice. I would like to give credit to them for formulating the research project the product of which is this thesis. I specifically appreciated their readiness to provide extensive feedback on my work, which included both the big-picture comments as well as more detailed technical assistance. They both provided an excellent researcher example for me to follow. I am grateful for their patience and encouragement during periods when my progress was less than consistent, for without that crucial element of supervision this dissertation might have probably never seen light.

I would like thank to the Economic and Social Research Council and the School of Management for financial support and assistance in the first three years of my candidature. Financial security was a key factor in allowing me to embark on this career path.

I wish to give thanks to Dr Shaun Harris and Dr Thomas Winfield for welcoming me to the Department and including me in the lunch-time crossword-solving sessions. I would also like to thank Dr Lauren Hayhurst, Dr Samantha Walker, and Dr David Shaw for graduating before me and leading by example. I have been incredibly fortunate to have had so many kindred spirits accompany me on this journey. Their understanding gave me strength and their successes were and still are a prime source of inspiration.

I owe a huge debt of gratitude to my family and loved ones for standing by me no matter what. Their unconditional love and support kept me on the right track all these years. Last but not least, I would like to thank my dog Sox for helping me stay fit both mentally and physically throughout my candidature. 


\section{List of Tables}

1.1 Types of transparency . . . . . . . . . . . . . . 5

2.1 Conditions for optimal disclosure policies under commitment in the J-L case . . . . . . . . . . . . . . . . . . . . . . . . . . 62

2.2 Conditions for optimal disclosure policies under discretion in the J-L

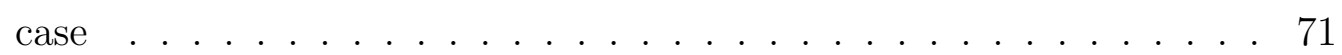

2.3 Optimal disclosure policies under commitment and discretion . . . . . 79

2.4 Conditions for optimal choice of policy regime . . . . . . . . . . . 81

2.5 Welfare outcomes and optimality conditions (hybrid case) . . . . . . 88

2.6 Welfare outcomes and optimality conditions (M-S case) . . . . . . . 88

2.7 Welfare outcomes and optimality conditions (J-L case) . . . . . . . 88

2.8 Summary of Chapter 2 results . . . . . . . . . . . . . . . . . . . . 92

3.1 Fixed cost. Equilibrium characterisation versus efficient benchmark (M-S with policy) . . . . . . . . . . . . . 136

3.2 Fixed cost. Equilibrium characterisation versus efficient benchmark (J-L) . . . . . . . . . . . . . . . . . . . . 143

3.3 Fixed cost. Equilibrium characterisation versus efficient benchmark (J-L with policy) . . . . . . . . . . . . . . . . . . . . 149

3.4 Linear cost. Conditions for a positive $\beta$ (M-S with policy) . . . . . 159

3.5 Linear cost. Conditions for a non-positive $\beta$ (M-S with policy) . . . . 162

3.6 Linear cost. Effect of policy on private information acquisition (M-S) 166

3.7 Linear cost. Conditions for a positive $\beta$ (J-L with policy) . . . . . . . 172

3.8 Linear cost. Conditions for a positive $\beta$, given $\rho=\rho^{*}$ (J-L with policy) 173

3.9 Linear cost. Conditions for a non-positive $\beta$ (J-L with policy) . . . . 174

3.10 Linear cost. Conditions for a non-positive $\beta$, given $\rho=-1$ (J-L with policy) . . . . . . . . . . . . . . . . . . 175

3.11 Linear cost. Effect of policy on private information acquisition (J-L) . 178

4.1 Public information acquisition. Expected loss outcomes for $\tau=\tau_{y}^{*}$

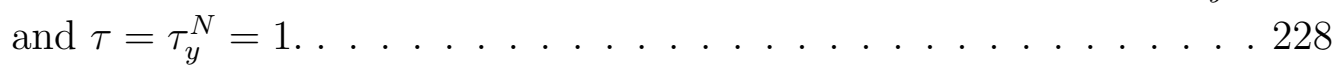

A.1 Summary of extensions and variations of the Morris and Shin framework241 
B.1 Conditions for optimal disclosure policies under commitment in the J-L case. Numerical example: $\sigma_{\varepsilon}^{2}=4, \sigma_{\phi}^{2}=1, Q=\frac{1}{4} \ldots 251$

B.2 Conditions for optimal disclosure policies under discretion in the J-L case. Numerical example: $\sigma_{\varepsilon}^{2}=4, \sigma_{\phi}^{2}=1, Q=\frac{1}{20} \ldots 256$ 


\section{List of Figures}

2.1 Hybrid payoff function. Degrees of coordination as functions of $\lambda$ for $r=0.85 \ldots \ldots \ldots \ldots \ldots \ldots \ldots$

3.1 Fixed cost. Shapes of expected equilibrium welfare in P-NE (M-S) . . 116

3.2 Fixed cost. Descriptions of equilibrium cases and CBT decisions (M-S) 121

3.3 Fixed cost. Descriptions of equilibria (M-S with policy) . . . . . . . 135

3.4 Fixed cost. Description of equilibria $(\mathrm{J}-\mathrm{L})$. . . . . . . . . . . . 140

3.5 Linear cost. Equilibrium and efficient information acquisition (M-S) . 157

3.6 Linear cost. Effect of changes in CBT on the existence of P-NE (M-S

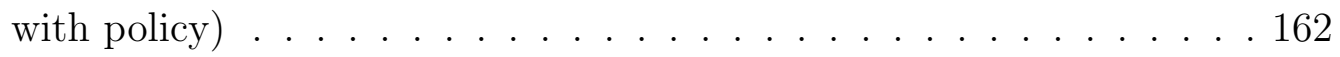

3.7 Linear cost. Optimal strategy for policy and CBT (M-S with policy) . 164

3.8 Linear cost. Disruption of a N-NE by CBT adjustment (M-S with policy) . . . . . . . . . . . . . . . . . . . . 164

3.9 Linear cost. Equilibrium and efficient information acquisition (J-L) . 170

3.10 Linear cost. Optimal strategy for policy and CBT (J-L with policy) . 176

4.1 M-S versus private information acquisition. The $\kappa$-function and the CB's isoloss for $\omega=\alpha=\gamma=1 \ldots 202$

4.2 Private information acquisition. The $\kappa$-function and the CB's isoloss for $\alpha=\gamma=1, \omega=0.8$ and $p=0.02$. . . . . . . . . . . 204

4.3 Private information acquisition at $\tau=0.58$ and $\tau=0.9$. The $\kappa$ function and the CB's isoloss for $\omega=\alpha=\gamma=1 \ldots 210$

4.4 Private information acquisition. The $\kappa$-function and the CB's isoloss for $\omega=\alpha=\gamma=1$ and $p=0.5 \ldots \ldots$. . . . . . . . . . . 210

4.5 Private information acquisition. The $\kappa$-function and the CB's isoloss for $\omega=\alpha=\gamma=1$ and $p=0.315 \ldots 21$. . . . . . . . . 211

4.6 Private information acquisition. The $\kappa$-function and the CB's isoloss for $\omega=\alpha=\gamma=1$ and $p \in\{0.3,0.315\} \ldots 21 \ldots \ldots . \ldots 21$

4.7 Private information acquisition. The $\kappa$-function and the CB's isoloss for $\omega=\alpha=\gamma=1$ and $p \in\{0.315,0.35\} \ldots 212 \ldots \ldots 2$

4.8 Public information acquisition. Existence of P-NE and N-NE . . . . . 221 
4.9 Public information acquisition. Optimal policy design for cases in which only the P-NE or the N-NE exists . . . . . . . . . . . . . 223

4.10 Public information acquisition. Inducing N-NE at given $p_{y}$ by setting

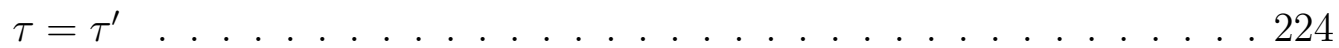

4.11 Public information acquisition. CB's expected loss in the P-NE and the N-NE as a function of $\tau \ldots \ldots$. . . . . . . . . . . . . 227

4.12 Public information acquisition. CB's expected loss in the P-NE and the N-NE and optimality of commitment to $\tau=1 \ldots 229$

4.13 Public information acquisition. CB's expected loss in the P-NE and the N-NE and commitment strategies . . . . . . . . . . . . 230

B.1 J-L case and commitment. Expected welfare under transparency regimes: $\sigma_{\varepsilon}^{2}=1, \sigma_{\phi}^{2}=4, Q=\frac{1}{16} \ldots \ldots . \ldots . \ldots 252$

B.2 Hybrid case and discretion. Shapes of the expected welfare function . 255

B.3 J-L case and discretion. Expected welfare under transparency regimes:

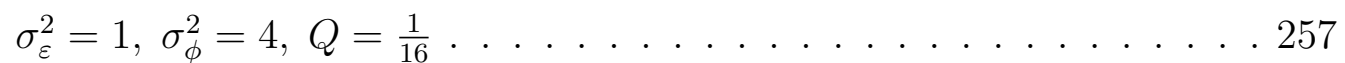

B.4 Policy performance in the M-S case: $\sigma_{\varepsilon}^{2}=4, \sigma_{\phi}^{2}=1, r=\frac{7}{10} \quad \ldots .258$

B.5 Policy performance in the J-L case: $\sigma_{\varepsilon}^{2}=1, \sigma_{\phi}^{2}=4, Q=\frac{1}{50} \ldots 259$

C.1 Model - Degrees of Coordination (M-S and J-L) . . . . . . . . . . 265

D.1 Private information acquisition. The CB's expected loss in the vicin-

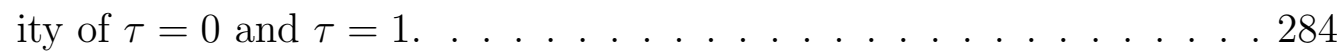

D.2 Private information acquisition. Critical and actual values of $\gamma$ as a function of $\tau$. . . . . . . . . . . . . . . . . . . . . . . 285 


\title{
List of Abbreviations
}

\author{
CB central bank \\ CBT central bank transparency \\ EDoC equilibrium degree of coordination \\ $\mathrm{FE}$ forecast error \\ FOC first-order condition \\ FOMC U.S. Federal Reserve's Federal Open Market Committee \\ GDP gross domestic product \\ J-L James-Lawler \\ M-S Morris-Shin \\ MSE mean squared error \\ N-NE non-purchasing Nash equilibrium \\ NE Nash equilibrium \\ P-NE purchasing Nash equilibrium \\ CEDoC collectively efficient degree of coordination \\ UK United Kingdom \\ US United States
}


To Eve 


\section{Introduction}

The past three decades have seen a trend toward greater transparency among the world's major central banks. Greater openness about the conduct of monetary policy reflects an established view in the central-banking community, which is shared by practitioners and theoreticians alike. Namely, there is consensus that managing expectations in the economy through central bank communication is in itself an important element of monetary policy. This view relies on the understanding that there is an inherent connection between central bank transparency, private sector knowledge, and effectiveness of policy.

Although there exists undeniable agreement on the importance of central bank communication, the question of how much transparency is the right amount of transparency remains open. In a similar vein, there is a variety of views on the precise ways in which openness is to be achieved as well as their relative effectiveness. Theoretical body of work on the subject provides less than unequivocal support for the idea that increased transparency is universally beneficial. The overarching aim of this thesis is to contribute to the ongoing conversation about the interaction of central bank communication and monetary policy effectiveness by providing a theoretical insight.

The principal aim of this study is to consider optimal policy design in the context of a narrow set of game-theoretic models. We define policy design as central bank's strategies for communication, instrument adjustment, and regime choice. The models we construct are based on seminal work by Morris and Shin $(2002,2018)$, and their key features include strategic complementarities and heterogeneity in private sector information. Each model variation that we consider is defined in terms of our assumptions about the information structure of the economy. We evaluate policy performance in terms of social welfare outcomes as well as its ability to eliminate informational inefficiencies.

Chapter 1 helps motivate the modelling approach we employ in the remainder of the thesis. In it, we provide real-world context for our theoretical study and give 
an overview of relevant theoretical literature. In Chapter 2, we extend the Morris and Shin (2002) model to include information stickiness in the private sector. In Chapter 3 we replace the sticky information assumption with considerations of costly private information acquisition. Finally, in Chapter 4 we construct a variation of the Morris and Shin (2018) market signal model that includes costly private and public information acquisition. 


\section{Chapter 1}

\section{Literature Review}

\subsection{Introduction}

The aim of the present Chapter is to provide context for the analysis undertaken in the remaining Chapters. The topics covered in this review of literature are organised around the key terms in the title of this thesis. We begin with more general considerations in central banking and then move on to more specific approaches to modelling policy regimes, coordination, and information.

Throughout this Chapter, we use the term monetary policy as an all-encompassing concept that includes a variety of central banking activities. The two primary aspects of monetary policy in our discussion are central bank transparency and instrument adjustment. First, we present a broad overview of practical considerations related to central bank communication in Section 1.2. We discuss the role of transparency in monetary policy as well as the ways in which it can be used as a policy tool. We then proceed to address the second aspect of monetary policy - instrument adjustment. In doing that, we adopt a narrower focus and restrict our discussion to theoretical literature that is concerned with the optimal choice of a policy regime.

In Section 1.4, we further narrow our focus and review a strand of literature that examines the social value of information. The discussion in this Section centres around a game-theoretic approach developed by Morris and Shin (2002). The Morris and Shin model conveniently isolates the role of public announcements in determining economic behaviour, thereby, providing a useful framework for analysing central bank transparency. We touch upon a number of key extensions to the Morris and Shin model that are of the highest relevance to the material presented in later Chapters. 
Finally, in Section 1.5, we cover three selected topics in information theory. This part of the review provides background to the specific modelling assumptions that set Chapters 2, 3, and 4 apart. Namely, we discuss the concepts of (i) sticky information, (ii) costly information acquisition, and (iii) signal value of market aggregates. Where possible, we refer to models that combine these information assumptions with the Morris and Shin framework.

\subsection{Central bank transparency: practical consid- erations and their implications for theory}

The phenomenon of central bank transparency emerged alongside increased independence and accountability of monetary authorities towards the end of the XX century. Since then the concept of greater openness has been increasingly embraced by central banks around the world ${ }^{1}$. Although central banks largely agree on the importance of transparency ${ }^{2}$, the actual means for achieving openness vary from bank to bank.

Taking into account the fact that the conduct of monetary policy is a multistage process, there is scope for transparency at every stage of policy-making. This argument has been developed by Geraats (2002), who suggests that the decision about increased openness may involve different motives depending on the aspect of monetary policy it relates to. With this in mind, Geraats distinguishes between five types of transparency: (i) political, (ii) economic, (iii) procedural, (iv) policy, and (v) operational. Every type refers to a different set of policy elements, thus a given central bank can be politically open whilst being opaque about its decision-making procedure. Table 1.1 lists examples of monetary policy elements relevant to each type of central bank transparency identified by Geraats coupled with selected evidence from a Bank of England questionnaire reported by Fry et al. (2000). Table 1.1 demonstrates that in practice transparency is achieved through a variety of channels, including explicit policy targets and objectives, publication of forecasts and their retrospective analysis, explanations of policy decisions, publication of voting records and minutes from committee meetings. Some of these channels, such as explicit targets and policy explanations, are widespread, whereas others, such as publication of voting patterns, are rarely used.

\footnotetext{
${ }^{1}$ The proportion of banks who publish explicit targets increased from $58 \%$ (of 84 banks) in 1990 to $95 \%$ (of 93 banks) in 1998 (Fry et al., 2000)

${ }^{2}$ Fry et al. (2000) report that $74 \%$ of 94 surveyed banks regard transparency as a "vital" or "very important" component of their monetary framework.
} 


\begin{tabular}{|c|c|c|}
\hline Type & $\begin{array}{l}\text { Elements of Monetary } \\
\text { Policy }^{1}\end{array}$ & $\begin{array}{l}\text { Evidence from a } 1998 \\
\text { Survey }^{2}\end{array}$ \\
\hline Political & $\begin{array}{l}\text { Policy objectives, prefer- } \\
\text { ences, explicit targets, po- } \\
\text { litical and instrument in- } \\
\text { dependence, accountability } \\
\text { contracts. }\end{array}$ & $\begin{array}{l}95 \% \text { of } 93 \text { central banks } \\
\text { publish explicit numeric } \\
\text { targets or monitoring } \\
\text { ranges. } 20 \text { out of } 94 \text { banks } \\
\text { have formal procedures for } \\
\text { government to overrule a } \\
\text { bank's decision. }\end{array}$ \\
\hline Economic & $\begin{array}{l}\text { Statistical and survey data, } \\
\text { models, structure of the } \\
\text { economy, forecasts. }\end{array}$ & $\begin{array}{l}39 \text { out of } 94 \text { banks pub- } \\
\text { lish forward-looking analy- } \\
\text { sis more than annually. } 32 \\
\text { out of } 94 \text { banks explain risks } \\
\text { to their forecasts. }\end{array}$ \\
\hline Procedural & $\begin{array}{l}\text { Policy rules, strategic con- } \\
\text { siderations, minutes, voting } \\
\text { records. }\end{array}$ & $\begin{array}{l}17 \text { out of } 94 \text { banks publish } \\
\text { minutes of policy meetings, } \\
6 \text { out of } 94 \text { banks publish } \\
\text { voting patterns. }\end{array}$ \\
\hline Policy & $\begin{array}{l}\text { Policy decisions, short-term } \\
\text { targets, explanation of deci- } \\
\text { sions, likely future actions. }\end{array}$ & $\begin{array}{l}76 \text { out of } 94 \text { banks provide } \\
\text { explanations of changes in } \\
\text { policy and } 15 \text { out of } 94 \\
\text { banks provide explanations } \\
\text { when policy remains un- } \\
\text { changed. }\end{array}$ \\
\hline Operational & $\begin{array}{l}\text { Implementation of de- } \\
\text { cisions, control errors, } \\
\text { unanticipated disturbances, } \\
\text { past forecast errors. }\end{array}$ & $\begin{array}{l}21 \text { out } 94 \text { banks always pro- } \\
\text { vide discussion of past fore- } \\
\text { cast errors. }\end{array}$ \\
\hline
\end{tabular}

${ }^{1}$ Using the conceptual framework of Geraats (2002);

${ }^{2}$ Evidence taken from Fry et al. (2000)

Table 1.1: Types of transparency

In what follows, we first discuss whether or not central bank transparency is unambiguously desirable as well as potential obstacles to achieving transparency. We then move one to explore the concept of intermediate degrees of transparency. Finally, we look at the use of transparency as a monetary policy tool, including forward guidance, as a more specific example.

\subsubsection{Desirability and feasibility of transparency}

Theoretical discussions of transparency in the literature are often accompanied by practical considerations of the various complications and trade-offs faced by the central bank when designing its communication policy. Disclosure of information can be conveniently analysed along two dimensions (Cukierman, 2009): (i) desirability 
and (ii) feasibility.

In a democratic setting transparency is essential to eliminate corruption and ensure accountability. In this broad sense central bank transparency is unambiguously desirable. Using Geraats' classification (Geraats, 2002), one can specify that this unambiguity applies to certain elements of political, procedural and policy transparency, such as clearly stated policy objectives and legal documents outlining the relationship between the central bank and the government. In other words, transparency about how the central bank operates and its role is seen as being healthy. Desirability of transparency about how the central bank makes its policy decisions, on the other hand, is subject to debate. Transparency in this narrower sense includes announcement of numerical targets for inflation, publication of forecasts, release of econometric models and policy rules as well as other information that is relevant to decision-making. The debate revolves around the effect that disclosure of such information may have on the effectiveness of monetary policy. An important contribution to this debate, that is also a central theme in this thesis, is presented in Section 1.4 below.

Mishkin (2004) proposes a simple test for evaluating desirability of transparency by stressing that transparency should be seen as a tool for achieving policymaker's goals, rather than an aim in itself ${ }^{3}$. In particular, the role of central bank communication is to help the central bank "to do its job" as well as strengthen public support for monetary authorities. The author claims that central bankers can identify the situations in which transparency is desirable by assessing, whether increased communication would serve the above mentioned purposes or whether it would just complicate the process of policy-making. In the latter case increased transparency can become excessive and undesirable. As a result, in certain instances, partial transparency can be the optimal approach.

In some cases, even if increased transparency is desirable, it is just not feasible. An argument made by Cukierman (2009) about measures of the output gap ${ }^{4}$ can be extended to other information involved in the monetary policy process; namely, explicit internal agreement is a precondition for external transparency. In other words, it is not feasible to release any data, until unique definitions and methods of measurement have been agreed upon within the institution. It should be noted, that publishing inconsistent data or conflicting estimates can be just as undesirable as

\footnotetext{
${ }^{3}$ Also in (Issing, 2005, pp. 68-69).

${ }^{4}$ Output gap is the difference between actual and potential output, the latter being a theoretical notion the measurement of which is subject to considerable disagreement within the economics profession.
} 
forcing internal agreement. The former can undermine the authority of the central bank and confuse the public, whereas the latter can lead to excessive simplification and exclusion of important information from consideration. This limitation applies to information that is subject to genuine uncertainty ${ }^{5}$ or debate, such as long-term forecasts, estimates with limited accuracy, variables that are subject to major revisions, intangible and directly unobservable measures, vague definitions and methods involving judgement. Use of such information in the central bank's decision-making process is often reflected in theoretical models. For instance, the presence of stochastic variables in the policymaker's information set is intended to represent the limited accuracy of internally used information.

Statements about the setting of policy instruments are also subject to the limitation described above. For instance, a central bank that places significant value on price stability adjusts its policy rate in order to prevent inflation from reaching an undesirable level or to return inflation to a desirable level. Decisions about instrument adjustment are based on a multitude of assumptions about the economy and the indirect relationship between the policy rate and the rate of inflation. These assumptions serve as a basis for structural models and Phillips curves, which reflect a certain level of understanding of relationships between economic variables. Although it is reasonable to assume that this level of understanding has been gradually rising over the past century, one can be certain that no central bank possesses full knowledge of the effect of adjustments in the policy rate on inflation. Thus, limited understanding of how the policy rate influences price stability restricts the scope for explanatory narrative in statements that accompany interest rate adjustments. In theoretical modelling limited knowledge about the economy and the effects of the policy rate adjustments are reflected in partial exclusion of the information about the policy transmission mechanism from the central banks information set. Similarly, the inability of the central bank to communicate the intricacies of the uncertainty surrounding the transmission mechanism can be introduced through restricting the accuracy of the private sector's knowledge of the transmission mechanism and imposing informational asymmetry between the central bank and the private sector on this subject.

\subsubsection{Implementing various degrees of transparency}

If it is possible to identify the optimal degree of transparency, it then becomes important to consider the limits of implementation, in particular, whether a specific degree of transparency - be it zero, full or partial - can be achieved in practice. It

\footnotetext{
${ }^{5}$ Here "genuine" uncertainty refers to Knightian uncertainty (Knight, 1921), which is unmeasurable, as opposed to quantifiable risk.
} 
is generally believed that the answer to this question is a negative one, since a wide range of limitations prevent the central bank from exerting perfect control over the degree transparency.

Firstly, the assumption of achievable zero transparency, or full opacity is hypothetical in nature. As pointed out by Svensson (2006), in any realistic scenario there is always at least some public information available. This view has a stronger grounding in the context of modern democratic countries, where transparency is an integral part of the democratic process. Issing (2005) illustrates the existence of public pressure on central banks by referring to a famous court case initiated by a US citizen against the Federal Reserve's Federal Open Market Committee (FOMC) ${ }^{6}$. The claimant expressed a need for greater transparency by demanding for the policy directive and minutes to be released immediately after every meeting of the FOMC. Thus, in a democratic country "monetary mystique" (Goodfriend, 1986) would always be criticised by the public. As far as theoretical models are concerned, this implies imposing an assumption of some non-zero minimum level of transparency.

Secondly, the concept of full transparency should be treated with certain caution. From a theoretical perspective central bank transparency can be defined as:

"the absence of asymmetric information between monetary policy makers and other economic agents" as well as "common knowledge about the information symmetry" (Geraats, 2002, pp. 533-534)

In practice full transparency can be defined as:

"a state in which the [central] bank fully and immediately transmits to the public all its private information about the economy, about its objectives and about the internal procedures that underlie the bank's policy decisions" (Cukierman, 2009, p. 3)

Hence, there can be a discrepancy between the central bank's effort to achieve transparency and actual achieved transparency. Although the central bank has a multitude of communication channels at its disposal, none of these means of transparency guarantee common knowledge amongst market participants (Cornand and Heinemann, 2008). Even if the central bank communicates all of its information, the latter might not reach all the market participants due to inefficiencies in information transmission. Furthermore, even if the revealed information is observed by all end-users in the market, it will still be subject to differences in interpretation,

\footnotetext{
${ }^{6}$ The court case and its implications for central bank transparency are discussed in more detail in Goodfriend (1986).
} 
judgement and competence of individual market participants. In reality these limitations are insurmountable (Issing, 2005), thus, practising full transparency might not necessarily lead to informational symmetry. Generally, in theoretical discussions transparency is only modelled in the context of a communication policy decision made by the central bank (latter of the two definitions above); however, a growing number of contributions also address the difficulty of achieving common knowledge. For instance, in Cornand and Heinemann (2008), market participants are assumed to interpret public information differently ${ }^{7}$, which eventually affects the central bank's decision about the communication policy.

Finally, achieving partial transparency of a specific level is difficult. On one hand, the resulting degree of transparency would tend to be below the desired level for the same reasons, as the ones that prevent the central bank from achieving full transparency. On the other hand, if partial transparency is interpreted as releasing the information to a specified fraction or subgroup of market participants, the resulting degree of transparency would tend to be greater than desired, primarily due to secondary dissemination of information (Cornand and Heinemann, 2008). This multiplier effect may arise for a number of reasons, including the following: (i) informed agents might have an incentive to spread the information revealed to them; (ii) the uninformed agents might have an incentive to actively seek out the information; (iii) the information might be revealed through the actions of informed agents; and (iv) the incentive for the media to disseminate information is not related to the market outcomes, but rather to the spreading of information itself. In the context of the modern world, transmission of information is facilitated by the multitude of media channels as well as the wide-spread use of the Internet, which makes it increasingly difficult for officials to withhold information or limit its audience. Thus, theoretical models that operate with intermediate degrees of transparency benefit from accounting for the multiplier effect and other imperfections of the communication mechanism.

\subsubsection{Transparency as a policy tool}

According to the expectationalist ${ }^{8}$ school of thought, managing expectations in the economy is an important element of monetary policy. This view is supported by two arguments: the self-fulfilling nature of market expectations as well as the limited ability of monetary authorities to achieve goals through adjustment of policy

\footnotetext{
${ }^{7}$ The authors extend their main model by assuming that an idiosyncratic random disturbance is added to the signal released to the fraction of agents who observe said signal.

${ }^{8}$ In Morris and Shin (2008) the term expectationalist is attributed to Charles Goodhart, who used it in personal communication.
} 
instruments.

The first argument relates to the term self-fulfilling prophecy coined by (Merton, 1948, p. 154) and defined as "a false definition of the situation evoking a new behaviour which makes the originally false conception come true" (italics in the original). In the financial markets the role of a "false definition" can be played by a forecast or a rumour that does not reflect the actual state of fundamentals, i.e. actual qualitative and quantitative data about the state of the economy and the markets. The idea of a self-fulfilling prophecy then implies that, if market participants believe the false forecast to be true, their actions may lead to the realisation of that false forecast. Merton describes a situation where a false rumour about insolvency results in a run on a hypothetical bank. Recent real-life examples include the collapse of Lehman Brothers in 2008 and the ongoing (as of the time of writing) European sovereign debt crisis. Lehman Brothers failed due to the escalating fall in counterparty confidence (Valukas, 2010), in other words, the default was caused by the fear of default. As far as the Eurozone government debt crisis is concerned, credit rating downgrades of peripheral European countries, which were "unfair" compared to the rating downgrades implied by the fundamentals, generated conditions under which the unreasonably low ratings were justified (Gärtner and Griesbach, 2012). The magnitude of market distortions that the mechanism of self-fulfilling prophecies can potentially create warrants the need for monetary authorities to monitor and manage expectations of market participants.

The second argument revolves around the limitations of conventional monetary policy instruments (e.g. short-term interest rate) in achieving targets and maintaining a stable financial environment. One of the sources of limited effectiveness of policy instruments is the policy lags, which can be broadly categorised depending on the stage at which they occur: (i) recognition, (ii) implementation, and (iii) effects. The first two types are sometimes referred to as inside lags, whereas the third type is referred to as the outside lag. The recognition lag, as the name suggests, is the time required to recognise the need for policy action and largely depends on the delays in production of relevant statistical data ${ }^{9}$. The implementation lag is the time required to make a policy decision and adjust the policy instrument, while the effects lag is the time required for the instrument adjustment to have an effect on variables of interest. Empirical evidence suggests that it takes over a year for in-

\footnotetext{
${ }^{9}$ As of the time of writing, Eurostat issues GDP figures 45 days (flash estimate) and 65 days (second estimate) after the end of the respective quarter. Inflation figures harmonised across European countries are released 15 days after the end of respective month. Release calendars for non-harmonised inflation figures vary between member states with delays starting at 5 days after the end of the respective month.
} 
strument adjustment to have its full effect on the rate of inflation ${ }^{10}$. The presence of lags exacerbates the uncertainty arising at every stage of the policy process, which diminishes the effectiveness of conventional policy instruments.

If credible, public announcements made by policy-making institutions are known to affect private sector's forecasts, which makes transparency a useful tool for coordinating expectations of economic agents. The ability of the central bank to align expectations of market participants is related to the phenomenon of the so-called sunspots.

Following Cass and Shell (1983), the term sunspots ${ }^{11}$ has been used in economics to denote a random factor that is not connected to economic activity directly, but nonetheless affects the economic outcome. Discussing self-fulfilling prophecies Azariadis (1981) concludes that, if enough agents believe that sunspots are good predictors of fundamental economic variables (such as prices and interest rates), they may choose to act in a way that would make their expectations bear out. Thus, if market participants are monitoring sunspot activity for forecasting purposes, it is possible to use sunspots as a policy instrument to adjust the paths of economic variables through self-fulfilling expectations. Morris and Shin (2008) identify a number of features possessed by sunspots which enable coordination of forecasts:

1. they are observed by all agents;

2. feature 1 is common knowledge among agents; and

3. having observed the sunspots, agents interpret them and act on them in the same way.

Based on these characteristics, Morris and Shin (2008) claim that transparent central banks have successfully adopted the role of sunspots. In particular, central bank's public announcements possess the features necessary for coordinating expectations and, although they may not necessarily be intrinsically related to determination of interest rates and prices, they have an extrinsic influence thereon.

In other words, public announcements made by the central bank can serve as a "focal point for beliefs" (Morris and Shin, 2002, p. 1522) regardless of whether

\footnotetext{
${ }^{10}$ Batini and Nelson (2001) confirm this observation using UK and US data for the period 19532001. (Fry et al., 2000, p. 50) report that perceived average length of time required for a change in instruments to fully affect inflation varies between 1 and 50 months in different economies.

${ }^{11}$ Cass and Shell define sunspots as "random phenomena that do not affect tastes, endowments, or production possibilities" (Cass and Shell, 1983, p. 94) and use the term to represent extrinsic uncertainty.
} 
these announcements include information about economic or financial variables. In practice, announcements made by monetary authorities tend to convey information about fundamentals. This information is monitored by market participants for decision-making purposes and has a direct (or intrinsic) effect on price-setting and interest-setting. However, the fact that every market participant knows that the announcement was observed by every other market participant offers additional indirect (or extrinsic) input into the decision-making process. Morris and Shin (2002) refer to this phenomenon as the dual role of public information and construct a model for assessing the social value of public information that allows for this dual role. This model will be discussed in more detail in Section 1.4.1 below.

Central bank's strategies for using communication as a monetary policy tool are often referred to as forward guidance. Campbell et al. (2012) make a useful distinction between Delphic and Odyssean forward guidance. The former refers to when a central bank publishes a forecast of macroeconomic performance as well as announces "likely or intended monetary policy actions based on the policymaker's potentially superior information about future macroeconomic fundamentals and its own policy goals" (Campbell et al., 2012, p. 2). Such forward guidance focuses on reducing the uncertainty experienced by the private sector, and does so by supplying private agents with forecasts from a reputable source, on the one hand, and a guide to the central bank's monetary policy stance, on the other. Odyssean forward guidance sees the central bank openly commit to a specific course of action. Provided that the central bank's reputation as a policymaker allows it to commit credibly, such forward guidance essentially eliminates any uncertainty private agents might have about monetary policy action within the horizon of guidance. In this case the uncertainty about the economic fundamentals remains, however, it can also be mitigated by publication of forecasts, as in the case of Delphic forward guidance. The obvious drawbacks of the arguably more rigid Odyssean approach are loss of monetary policy flexibility as well as vulnerability to time inconsistency, which echoes Cukierman's desirability and feasibility arguments (Cukierman, 2009).

Having discussed a variety of considerations surrounding central bank transparency, we briefly turn to a different aspect of monetary policy in the following Section. Namely, we look at monetary policy regimes. 


\subsection{Monetary policy regimes: commitment ver- sus discretion}

There exists a vast theoretical literature that is concerned with the optimal choice of a monetary policy regime. The two main regimes that are typically considered are commitment and discretion. Broadly speaking, commitment is a transparent regime whereby the public is made aware of how monetary policy decisions are made, and once the policy framework is revealed it is adhered to indefinitely. Meanwhile, the regime of discretion implies that in every period monetary authorities adjust the policy instrument using all information available at that time so as to maximise an objective function relevant at that time. Discretion implies that the private sector may not be able to predict the actions of the policymaker with certainty, that the latter has considerable freedom of action and that the information set and objectives of monetary authorities can change over time. The discussions of relative benefits and disadvantages of the two regimes in the literature vary in terms of contexts, methods, assumptions as well as the definitions of commitment and discretion.

In the literature on central banking monetary policy regimes are typically evaluated in terms of inflation and real economic performance, as well as the effect monetary policy conduct has on private sector expectations. A particular strand of literature presents an argument against discretion that centres around a somewhat counter-intuitive result: although under discretion the policymaker chooses the best action given the current situation, the social welfare outcome is likely to be suboptimal due to higher levels of average inflation ${ }^{12}$. Moreover, ceteris paribus commitment to a rule would typically yield a superior outcome. In the terminology of Kydland and Prescott (1977), this result is due to the misalignment between optimal and time-consistent policy. For policy to be time-consistent, future policy plans formed in the current period, have to remain optimal when future periods come; meanwhile optimal policy is simply the best response, given the objective function. According to Kydland and Prescott, if there is no mechanism - institutional or legal, for instance - that could ensure that the policymaker makes socially optimal policy choices in future periods, optimal policy becomes time-inconsistent. In this context commitment to a rule is seen as a mechanism for preventing timeinconsistency and the difference in the social cost of inflation under the two regimes reflects the value of being able to credibly commit (Barro and Gordon, 1983). The main implications of time-inconsistency for monetary policy design are of limited

\footnotetext{
${ }^{12}$ Here high inflation in terms of levels is assumed to be detrimental to public welfare, however, it should be noted that undesirability of high yet stable levels of inflation and the definition of high inflation are both subject to debate in economic literature.
} 
relevance to the model presented in the following Chapters; however, this strand of literature provides an array of useful approaches to the formal treatment of monetary policy regimes in strategic games.

As far as desirability of commitment is concerned, a pre-specified policy rule implies a loss in flexibility, which can manifest itself in several ways. Firstly, a rule implies a fixed structure of the information set used for adjusting the policy instrument. Be it a structural change in the economy or a temporary shock, if the variables concerned are outside the pre-determined set of variables of the information set, they cannot be taken into account without abandoning the rule. Thus, even if new information is identified and its importance for the policy decision recognised, it cannot be incorporated into the decision-making process. Secondly, any unanticipated shock cannot be adequately addressed, especially, if reacting to it requires an unconventional approach. For instance, in the context of inflation and output, these constraints may potentially create a trade-off between the ability to achieve lower inflation and the ability to stabilise the economy in the event of a shock. In this case, the optimal balance depends on the importance of economic stability relative to price stability and the variance of shocks that hit the economy. In practice commitment to the alternative objective of stable inflation can put output performance at risk if inflationary pressures arise during a period of exceptionally weak economic growth, in which case monetary authorities may temporarily favour the objective of stabilising the economy. It should be noted that the relevance of the "limited flexibility" argument largely depends on the breadth of the adopted definition of commitment.

A rather narrow definition of commitment assumes the announcement of a policy rule. The announced rule can take the form of an ad hoc or an optimally-derived expression. An ad hoc rule is fixed and exogenous, which makes it straightforward and easily understandable by the public. However, a fixed rule leaves no scope for reacting to extraordinary circumstances and will typically not be optimal in a stochastic environment, given the objective function. Optimal rules can be derived for every future period in advance (optimal commitment) or there can be a single optimally-derived time-invariant rule followed in every period (timeless perspective $)^{13}$. Generally, these rules are state-contingent, in that the instrument-setting depends on the realisation of some variable with the form of the rule remaining the same. Alternatively, rules can be specified in terms of target values or condi-

\footnotetext{
${ }^{13}$ See Woodford (1999) and McCallum (2005) for brief discussions on the distinction between optimal commitment and a timeless perspective commitment.
} 
tions for some macroeconomic variables ${ }^{14}$. The variety of possible rule specifications is well-captured by the broad definition of monetary policy rules as "a prescribed guide for monetary policy conduct" given by Svensson (1999). In practice, however, central banks do not announce policy rules, the latter remaining the subject of discussion in academic literature. Arguably, the main reason for policy rules not being implemented in practice is the difficulty of deriving such rules in the absence of professional agreement about the appropriate specifications of macroeconomic models (McCallum, 1997). Thus, drawing the distinction between commitment and discretion in the behaviour of actual central banks requires a broader definition of commitment that is not restricted to policy rules. McCallum (1993) argues that in practice commitment can be identified by the following two features of a central bank's response to economic developments - the response should be systematic ${ }^{15}$ and it should take account of how private-sector expectations are formed.

At this point it is useful to consider the difference in modelling discretion and commitment. One way in which the distinction can be drawn is the order of events in a given time period, for instance, as it appears in the time-inconsistency literature. Under discretion, the policymaker aims to maximise the objective function for the current period, given all available information, including the expectations and actions of the private sector, as perceived by the policymaker. Thus, instrument adjustment as a function can take any form, depending on the objective function and the variables known to the policymaker at the time when the decision is made. The private sector, in turn, attempts to predict the instrument adjustment based on the information it has about the state of economy as well as its understanding of the policymaker's objective function, the monetary policy decision-making process and the structure of the economy. The sequence of events is as follows:

1. the policymaker reveals its preferences;

2. the private sector forms expectations of fundamentals and the instrument adjustment, given its information set;

3. the policymaker adjusts the instrument, given its information set, which includes expectations of private sector actions;

4. any unanticipated shocks are realised;

5. outcomes are determined, given monetary policy and private sector actions.

\footnotetext{
${ }^{14}$ See, for instance, Svensson (1997) and Section 7.3.5 in Walsh (2010).

${ }^{15} \mathrm{McCallum}$ expands the definition of commitment proposed by Taylor (1993) who suggests that rule-like behaviour is systematic, meaning "methodical" and "according to a plan".
} 
As far as commitment is concerned, the policymaker announces the rule it intends to follow from the offset, eliminating the need for the private sector to derive the expression for optimal instrument-setting from the objective function. Thus, step 1 in the above sequence is replaced by the policymaker revealing the policy rule and step 2 involves the private sector forming expectations of instrument adjustment based on the announced rule. Although the time-inconsistency literature evaluates policy regimes in the context of multi-period models, the above sequence of events equally applies to a single-period setup such as the one introduced in the next Chapter.

Above, the difference between commitment and discretion is discussed in relation to monetary policy, however, this distinction can also be applied to the choice of a disclosure regime. Faust and Svensson (2001) discuss the optimal degree of central bank transparency in a multi-period model under two alternative assumptions about the way disclosure policy can be implemented. The authors assume that the policymaker can either commit to an endogenously chosen quality of its announcements "once-and-for-all" or choose the optimal degree of transparency in every period. Under both regimes, the announcement reveals to the public how closely monetary policy actions are linked to monetary outcomes and the informational value of the announcement depends on the chosen degree of transparency. Optimal setting of disclosure policy is a common theme in the next two Chapters, and the central bank is assumed to always commit to the chosen transparency regime. The assumption of commitment is arguably more realistic, since, as Faust and Svensson (2001) point out, the mechanisms that facilitate commitment to high or low levels of transparency are well-established in practice.

Monetary policy in the form of instrument adjustment is a key element in all models presented in the following Chapters of this thesis. The distinction between commitment and discretion is particularly relevant to the discussion in Chapter 2, whereas Chapters 3 and 4 are limited to the analysis of commitment only. Having covered the subject of central bank transparency and monetary policy, we now turn to coordination games. The latter provide a useful framework for exploring how social value of information interacts with policy design.

\subsection{Social value of information and coordination}

Morris and Shin (2002) introduced an approach for analysing central bank transparency that isolated the value of public announcements for the market (or the economy) as a whole. The model proved to be tractable and readily extendible, 
which resulted in a strand of literature that explored the use of public information and its value in a variation of economic and financial contexts. Being game-theoretic in nature, the Morris and Shin approach is essentially an application of a class of games referred to as 'global games'. Thus, before discussing the Morris and Shin model, we first briefly discuss the notion of global games.

The concept of global games - as well as the term itself - was introduced by Carlsson and van Damme (1993) as a way of removing or reducing the multiplicity of Nash equilibria that often arise in non-cooperative complete information games ${ }^{16}$. To clarify the terminology:

- in a complete information game, all payoffs are known by all players with certainty and are, in fact, common knowledge, i.e. all players know the payoffs, they also know that they all know the payoffs, that they all know that they all know the payoffs, and so on ad infinitum.;

- in a non-cooperative game, there exists no mechanism for players to cooperate even if agreeing on a strategy would yield a higher payoff for everyone;

- a Nash equilibrium is a solution to the game such that no player has the incentive to change their action, given that other players' actions remain unchanged.

It is not uncommon that no single solution can be identified in a non-cooperative complete information game unless additional assumptions are imposed. If such a game is used to model economic decision-making, multiplicity of equilibria is an inconvenient result for policy analysis. Carlsson and van Damme demonstrate that by relaxing the assumption of complete information it is possible to single out a Nash equilibrium ${ }^{17}$. The authors argue that, in addition to providing a mechanism for equilibrium selection, their approach also enhances the degree of realism in relation to informational assumptions. Namely, they suggest that accounting for possible uncertainty about payoffs is a more realistic approach.

More specifically, Carlsson and van Damme (1993) relax the common knowledge assumption by assuming that players observe the payoffs with a noise and are aware of the fact that their observations are noisy. Until actual payoffs are revealed, a player does not know the precise game being played and, thus, is choosing a strategy for a whole class of games, which is the reason for the use of the term global. Morris

\footnotetext{
${ }^{16} \mathrm{~A}$ concise yet comprehensive overview of the global games approach and its economic applications can be found in Morris (2008).

${ }^{17}$ Carlsson and van Damme analyse a two-player two-action $(2 \times 2)$ game, and the identified Nash equilibrium is, in fact, what Harsanyi and Selten (1988) refer to as the risk-dominant solution.
} 
and Shin (1998) apply the noisy-observation approach to a financial model of currency attacks, which, as conventional wisdom suggests, are self-fulfilling in nature. The authors note that, under the common information assumption, a formal model of currency attacks produces multiple equilibria, which impedes effective analysis. In this case, every market participant observes the fundamentals with zero noise and knows that every other market participant observes the fundamentals without any noise. Once the assumptions are changed, every market participant observes the fundamentals with an individual noise and can no longer be certain that others observe the same information. The authors stress that it is this uncertainty about other participants' beliefs that is central to speculative attacks and demonstrate that the noisy-signal modelling assumption is sufficient to yield a unique equilibrium. Morris and Shin (2000) generalise the methodology of the global games approach, expand its application to a wider range of economic scenarios, and advocate for its use in macroeconomics.

Equilibrium uniqueness in the Morris and Shin (1998) model crucially allows for policy analysis. In the model, the central bank ${ }^{18}$ is one of the players in the game and decides whether or not to defend a fixed exchange rate. Relaxing the complete information assumption, leads to the determination of a uniquely optimal strategy for the bank. A more general implication for monetary policy is that, in order to stabilise a market that is on the edge of a currency crisis, it is optimal for the central bank to make 'a prominent public announcement' (Morris and Shin, 1998, p. 595). Authors argue that a clear announcement about market fundamentals, in that case, restores the transparency about the situation, thereby, bringing the market as close to the complete-information scenario as possible. Their conclusion draws attention to the importance of public announcements for strategic behaviour.

\subsubsection{Coordination and the Morris and Shin framework}

As already mentioned above, Morris and Shin (2002) discuss the value of public announcements in the context of a strategic game played by a large population of agents and a social planner. The authors show that under certain circumstances better public information is, in fact, detrimental to social welfare, which implies that transparency is not universally beneficial. Although the result of Morris and Shin's analysis is of primary importance, the model itself deserves a closer look.

Morris and Shin begin by distinguishing between public and private information. Public information is available to all agents, whereas private information is

\footnotetext{
${ }^{18}$ Referred to as the government by Morris and Shin.
} 
possessed by one agent only. As already mentioned above, there is a social planner (the central bank) and an infinitely large population of individual agents (the private sector). Individual agents engage in a simultaneous move game with the aim of minimising their loss. The shape of the loss function is such that there are two sources of loss and, hence, agents are governed by two motives when making their move. Firstly, agents aim to align their actions with the fundamentals (represented by an underlying state variable). Secondly, agents aim to align their actions with those of other agents. The latter motive is referred to as the coordination motive.

Coordination of actions in the market is central to the Morris and Shin result. The precise nature of coordination in their model stems from the actions of individual market participants being strategic complements ${ }^{19}$. They are complements, because a change in action by one agent creates an incentive for other agents to change their actions in the same direction. They are strategic - in the sense first used by Bulow et al. (1985) and Cooper and John (1988) - in that the marginal payoff of a given agent is affected by changes in action strategies of other agents, which in turn impacts the strategy of the given agent. Strategic complementarity in the payoff structure of the model creates an incentive for market participants to coordinate their actions. However, Morris and Shin assume that individual agents play a simultaneous-move non-cooperative game, which means that agents cannot collaborate with each other to achieve a more beneficial outcome and have to decide on their actions simultaneously. Thus, individual agents are forced to achieve coordination by using the information available to them and forming expectations about the action profile of the market.

Morris and Shin point out that this setup is reminiscent of the beauty-contest scenario described by Keynes (1936), in which newspaper readers are asked to choose the six prettiest faces from a hundred photographs with prizes awarded for choosing photographs with the most votes. As Keynes explains, the competitors face the incentive to coordinate their opinions in order to win the prize, thus they are not choosing the photographs that they like the most, but rather the ones they think others will like. Since all competitors are following the same strategy, in effect, they are trying to predict "what average opinion expects the average opinion to be" (Keynes, 1936, p. 156). Keynes used this anecdotal example to illustrate investor behaviour in the financial markets, where the importance of accurately predicting market valuations often takes precedence over predicting the actual yields. Follow-

\footnotetext{
${ }^{19}$ Morris and Shin (2002) briefly look at a setup where the actions of individual agents are strategic substitutes and find that this assumption inverts the equilibrium behaviour of agents, as compared to the case with the original complementarity assumption.
} 
ing this analogy, Morris and Shin refer to the incentive to coordinate in their model as the beauty contest motive. Morris and Shin show that a strong beauty contest motive diminishes the incentive for individual agents to act upon their predictions of market fundamentals, which parallels Keynes's original argument.

The nature of coordination in the Morris and Shin model is such that it is only beneficial at the level of individual agents and yields no benefit to the society as a whole. Coordination is achieved through observing the public announcement, which acts as a sunspot. Hence, public information serves two purposes: (i) enhancing the information set of individual agents, and (ii) providing means for coordination. The authors find that, due to the coordination motive individual agents attach excess importance to public announcements whilst underestimating the value of their private information. If a forecast published by the central bank is of relatively poor quality, overreaction to it can be harmful. Thus, social value of public information depends on quality of information available to the authorities relative to the quality of private information possessed by individual agents. If the private sector has access to high quality information, the central bank should refrain from making announcements unless sufficiently high quality of disclosed information can be guaranteed.

The above result is scrutinised in a comment by Svensson (2006), who suggests that Morris and Shin's analysis supports the argument in favour of transparency. Svensson points out that the detrimental effect of increased transparency in the Morris and Shin model kicks in when the quality of public information is significantly ${ }^{20}$ lower compared to private information. Moreover, individual agents need to value coordination more than successfully aligning their actions with the fundamentals. Although the author refrains from discussing the relevance of a strong coordination motive, considerable attention is given to the plausibility of quality parameters that lead to harmful transparency. Svennson claims that public information is unlikely to be less accurate than private information and refers to empirical evidence from Romer and Romer (2000), who compare Federal Reserve and private sector forecasts and find that Federal Reserve has a considerable informational advantage over commercial forecasters. However, Morris and Shin (2005) point out that commercial forecasters are not representative of "typical economic agents", whose information is gathered through direct observation of the economic environment and, thus, may be more accurate than central bank's information that is based on aggregated statistics. Svensson (2006) proceeds by defining a conservative benchmark case as the situation where the quality of public announcements is equal to the quality of agents' private information; however, even the benchmark case violates the conditions derived by

\footnotetext{
${ }^{20}$ At least 8 times lower.
} 
Morris and Shin. Furthermore, the author argues that, since in realistic scenarios there is always at least some public information of positive value available (i.e. full opacity is unlikely to be practiced), it may be that the Morris and Shin requirement for detrimental transparency cannot be met in practice.

Morris et al. (2006) accept Svensson's observations and demonstrate that the original Morris and Shin result holds in a variation of the original model. Morris et al. present a two-player game and introduce correlation into information sets of agents as well as the central bank. The authors address Svensson's critique of the condition for harmful transparency by showing that in the modified setting better public information can be detrimental even if the quality of public information is higher that the quality of the private information of individual agents. Similarly, Morris and Shin (2005) demonstrate that, if the original model is adjusted to allow for the endogenous nature of central bank's private information, precision of public information can fall below the precision of private information available to the private sector. The latter conclusion casts doubt on the validity of the conservative benchmark notion discussed by Svensson. In the light of Svensson's critique as well as the insights obtained by extending the original framework, Morris et al. (2006) call for further scrutiny and systematic investigation of the Morris and Shin model as well as the quantitative significance of its results.

\subsubsection{Extensions of the Morris and Shin framework}

The model introduced by Morris and Shin (2002) is attractive due to its simplicity and tractability. The authors identified a particular advantage of the model in its ability to produce a unique equilibrium regardless of the parameter values. As a testament to its virtues, the Morris and Shin framework has been repeatedly extended and modified by the original authors (Morris and Shin, 2002, 2005) as well as other scholars (Angeletos and Pavan, 2007b; Cornand and Heinemann, 2008; James and Lawler, 2011, 2012a,b) to further investigate the social value of public information. A summary of extensions and variations of the Morris and Shin model are presented in Appedix A.1. In what follows some of these extensions are briefly discussed.

Angeletos and Pavan (2007b) discuss the coordination motive in more detail in the context of a more general model with an extended payoff function. Agents' final payoffs are now additionally affected by the average action in a way that does not alter the original Morris and Shin game at an individual level. At the level of the society, however, coordination becomes desirable. In the Morris and Shin framework the coordination motive disappears at the aggregate level, meaning that alignment 
of actions itself yields no social benefit. Angeletos and Pavan argue that in order to discuss informational inefficiencies, one must first derive an efficient benchmark defined as a situation where all available information is used in a welfare-maximising way $^{21}$. The authors proceed by identifying two concepts central to their discussion: (i) equilibrium degree of coordination that reflects the private value of aligning actions, and (ii) socially optimal degree of coordination that reflects the value that society assigns to coordination. Using these terms the Morris and Shin model can be described as a special case characterised by a zero socially efficient degree of coordination and a positive equilibrium degree of coordination. Angeletos and Pavan conclude that when the two degrees of coordination are not equal to each other the use of information within the model is inefficient. If the equilibrium degree of coordination exceeds the socially optimal degree of coordination, the action profile of the population is described by heightened inertia and volatility. The former is caused by a weak reaction to the change in fundamentals, whereas the latter is due to overreaction to the common noise contained in public announcements. Thus, the extended model analysed by Angeletos and Pavan sheds light on the source of informational inefficiencies within the Morris and Shin framework.

In the original paper Morris and Shin (2002) touch upon the possibility of the central bank purposefully varying the quality of public information in attempt to achieve a superior welfare result. The model shows that announcements of very low quality may discourage inefficient coordination, making precision of disclosed information a potential policy tool. Cornand and Heinemann (2008) introduce an additional transparency tool into the Morris and Shin framework. The authors argue that the central bank can prevent agents from overreacting to public information by removing the feature of public announcements that allows these announcements to act as sunspots. As mentioned above, the main characteristic of sunspots is that they are observed by all agents; thus, once public information is only made available to a fraction of agents, its usefulness for coordination is greatly reduced. Cornand and Heinemann refer to this tool as the degree of publicity, which is defined as the proportion of agents who observe the announcement. The Morris and Shin result becomes a special case of the extended model with the degree of publicity equal to unity. In addition to affecting the alignment of actions across agents, the degree of publicity also affects the relative distribution of their payoffs by impacting "differentially on those who observe the signal and those who do not" (James and Lawler, 2012b, p. 555).

\footnotetext{
${ }^{21}$ Hellwig (2005) introduces the notion of an efficient benchmark in a discussion of welfare effects of public information within a model of monopolistic competition and refers to it as the "decentralized information optimum".
} 
Cornand and Heinemann observe that it is optimal for the central banker to restrict the number of agents who receive the announcement if the quality of public information is relatively low. The authors proceed by considering the case where the policymaker chooses both the optimal degree of publicity as well as the optimal precision of the announcement. It appears that, if the central bank has the ability to set the degree of publicity optimally, then the disclosed information should be of highest possible quality; if, however, the choice set is limited to full publication (i.e. degree of publicity equal to unity), the Morris and Shin (2002) conclusion applies. Cornand and Heinemann take a stance that as a transparency tool the degree of publicity is more efficient and preferable to varying the precision of public information.

The original Morris and Shin model as well as the extensions discussed above focus on the role of transparency in addressing the issue of inefficient use of information within the private sector. It can be shown, however, that the way in which information is used can be influenced through direct policy intervention. Using a more general model Angeletos and Pavan (2007b) show that informational efficiency can be achieved by an appropriately designed system of Pigouvian taxes. A tax rate that is contingent on the ex post average action can discourage overreaction to public information and yield a welfare result equivalent to that of the efficiency benchmark, as defined above. When such a tax system is applied to the Morris and Shin model increased transparency becomes invariably beneficial. James and Lawler (2011) consider an alternative type of policy intervention and their model yields the opposite implication for disclosure policy.

James and Lawler (2011) modify the original model by allowing the central bank to intervene into the market and directly influence fundamentals. The central bank commits to a rule that is conditional on the central bank's private information. Before adjusting its policy instrument the central bank makes a public announcement based on its private information, however the bank is free to lower the quality of information contained in the announcement ${ }^{22}$. The authors find that, if the policy rule is designed optimally, given the quality of information available to both the private sector as well as the central bank itself, better public information is detrimental for welfare. This result supports the anti-transparency argument and strengthens the original findings made by Morris and Shin. Furthermore, James and Lawler show

\footnotetext{
${ }^{22}$ The concept of noisy announcements in the context of central bank transparency was formally introduced by Cukierman and Meltzer (1986) and later extensively used in the transparency literature.
} 
that in the presence of optimal policy complete opacity eliminates the inefficiency that arises from the beauty-contest motive. Hence, optimal policy intervention combined with zero disclosure enables the achievement of an efficient welfare outcome.

James and Lawler (2012b) show that the above result remains valid in the absence of the beauty contest motive. The authors use an alternative formulation of the payoff function that is, in the terminology of Angeletos and Pavan (2007b), characterised by an equilibrium degree of coordination that is lower than socially optimal. Their 'alternative' payoff function displays strategic complementarity in agents' actions making it directly comparable with the Morris-Shin payoff structure. The authors show that, in the absence of policy intervention by a market regulator, individual agents place excess importance on their private information, as the coordination motive is not strong enough to induce the socially optimal level of attention to public announcements. James and Lawler note that the inverse behaviour of market participants in equilibrium (relative to the original Morris and Shin result) demonstrates, that it is not the strategic complementarity per se that causes a non-optimal degree of attention to public information, but rather the misalignment of the two degrees of coordination, as suggested by Angeletos and Pavan (2007a).

The formulation of the policy rule in James and Lawler (2012b) is also different and includes a transmission mechanism shock known to the policymaker, but not the private sector. In contrast to James and Lawler (2011), here the authors follow Cornand and Heinemann (2008) and use the degree of publicity as a transparency tool. They find that in presence of optimally designed policy complete opacity yields an efficient outcome. Using the same setting James and Lawler investigate how the implications for transparency are affected if the signalling role of policy is incorporated into the model. For this purpose the central bank is assumed to reveal its instrument-setting before individual agents make their decisions. However, the private sector can infer central bank's private information from the observed adjustment of the instrument only to a certain extent, since the policy transmission shock remains unobserveble. In this modified game zero or partial disclosure is superior to full transparency, which strengthens the conclusions obtained in James and Lawler (2011)

James and Lawler (2012a) expand the model even further by considering the impact of heterogeneity in the quality of private information. The authors allow for two levels of quality of private information and, thus, distinguish between betterinformed and less well-informed agents. On one hand, the existence of two types of agents strengthens the coordination motive, on the other hand, it also strengthens 
the beneficial effect that better public information has on the information set of individual agents. As a result, the anti-transparency result of James and Lawler (2011) does not fully apply to this model. Given that the central bank adheres to an optimal policy rule, zero, partial or full disclosure can be optimal depending on the specific combination of the parameter values. However it can be shown that, the greater the difference in the information quality within the private sector the more likely it is that full opacity is optimal. The authors show that optimally designed policy rule combined with an optimally chosen degree of publicity no longer yield the efficient outcome. An optimally designed Pigouvian tax scheme, however, enables efficient use of information, meaning that findings of Angeletos and Pavan (2007b) are unaffected by the existence of two agent types.

Morris and Shin (2005) discuss the potential conflict between the two roles of the central bank: (i) an active shaper of market expectations, and (ii) and vigilant observer of market expectations. The former refers to the need for the central bank to manage expectations, since expectations provide an important link between monetary policy and the economy. The latter role entails gathering economic and financial data and using it for deciding on an appropriate policy course. The authors argue that the more the central bank influences market expectations the less informative market indicators get, since they are no longer representative of fundamentals and instead reflect central bank's own actions. Thus, the central bank faces a trade-off between the benefit of aligned expectations and monetary policy decisions that are adequate and timely.

To investigate the welfare effect of shaping market expectations through public announcements Morris and Shin (2005) modify their original framework by endogenising public information. The authors introduce dynamics into the model in a way that does not alter the strategic game for individual agents. The central bank's information set includes noisy information about the average action chosen by all agents in previous periods. If the central bank chooses to disclose its private information, individual agents take it into account, which has an effect on the average action. In the next period the information that the central bank gathers partially reflects the public announcement made by the central bank in the previous period. Morris and Shin find that under full transparency the quality of central banks information is lower than under full opacity. The result obtained by the authors implies that policy decisions made under full transparency address changes in fundamentals to a lesser extent than could be possible should central bank's information be withheld from the public. Thus, Morris and Shin (2005), highlight the importance of taking the dynamics of information revelation into account when considering the 
welfare effects of greater transparency and caution against overreliance on a static game such as their original model.

The Morris and Shin framework serves as the unifying basis for the scenarios analysed in the following Chapters of this thesis. The scenarios differ in the precise ways in which the original framework is extended. Key distinguishing features relate to the assumptions made about information quality, acquisition, and dissemination. The remainder of this literature review will narrowly focus on selected informationrelated themes and how some of them relate to the Morris-Shin framework.

\subsection{Selected topics in information}

\subsubsection{Sticky information}

The assumption of asymmetric information among economic agents is widely used in theoretical models due to its intuitiveness and realism. The nature of heterogeneity in information sets can be given exogenously or determined endogenously. In the both cases, one of the mechanisms that can produce asymmetric information is the reluctance or inability of a subset of agents to incorporate new information in the decision-making process, or information stickiness.

In the context of economic models, the term sticky information was introduced ${ }^{23}$ by Mankiw and Reis (2002) to describe slow diffusion of information about macroeconomic conditions through the population. Although, in their paper, Mankiw and Reis abstract away from the task of providing microfoundations for their approach ${ }^{24}$, the authors identify several possible interpretations of underlying causes. In brief, rigidities in the process of updating information sets can arise for a number of reasons, which include:

- the cost of acquiring new information,

- the cost of processing new information, and

- the cost of acting on new information.

Information is a commodity and it is costly, if not in monetary terms, then in terms of opportunity cost and leisure foregone. Information that is particularly valuable

\footnotetext{
${ }^{23}$ The term sticky information was originally coined by Von Hippel (1994) in the context of innovation and management; and the need for macroeconomic models to incorporate informationprocessing rigidities was recognised by Sims (1998).

${ }^{24}$ Microfoundations for information stickiness produced by inattentiveness of consumers and producers can be found in Reis (2006a) and Reis (2006b), respectively.
} 
for making economic decisions is likely to be produced by institutions who charge fees for subscriptions or one-off usage. However, even publicly available information that does not require payment of explicit fees is inaccessible without incurment of implicit costs, such as on transport or internet access.

Once accessed, new information needs to be processed. This may mean organising and elaborating new information - or thinking (Begg and Imperato, 2001) which merely requires time and effort. Alternatively, information processing may require specialised skills and expertise, which implies payment of fees or hiring personnel. For instance, although public sector institutions freely disclose the information about the state of the economy, understanding this information and its implications for economic decisions requires at least a basic understanding of economic principles.

Having acquired and processed new information, economic agents do not necessarily act on it. Re-optimising and implementation of decisions may involve incurring expenses, such as the so-called menu costs (Mankiw, 1985) as well as other managerial and customer costs (Zbaracki et al., 2000). In case of high-profile institutions that are scrutinised by the media, implementing changes can also have an adverse effect on reputation or credibility. Individual economic agents may be reluctant, for instance, to switch service providers due to having to pay a fee for a new contract, even if new information indicates that staying with the old provider is suboptimal. All of the above rationales can lead to inactivity or slow adjustment on part of agents. Endogenisation of information stickiness would require a formal treatment of one these rationales.

Formally the concept of 'stickiness' translates into the assumption that a fraction of the population acts on outdated information, whereas the rest of the economic agents update their information sets and use new information for making decisions. Although Mankiw and Reis originally used the sticky information assumption in the context of a multi-period model of price-adjustment, the concept is readily applicable to a wider range of models, including one-period frameworks. For instance, Hahn (2012) introduces the stickiness assumption into a one-shot price-adjustment game. However, the focus of Hahn's analysis is assessing the desirability of central bank transparency, rather than identifying the effects of sticky information per se.

Although the assumption of sticky information can render equilibrium derivation and other workings complicated, it appears that dynamic sticky-information models perform well when it comes to explaining the paths of macroeconomic variables. Mankiw and Reis (2002) demonstrate that a sticky-price model can explain certain 
stylised facts about the interaction of monetary policy, inflation and output, that other commonly used models fail to account for. Information stickiness has also been shown to help explain the dynamics present in labour markets (Mankiw and Reis, 2003), consumption (Reis, 2004) and business cycles (Mankiw and Reis, 2006). The sticky-information model also performed well with respect to microeconomic data on consumption behaviour of households (Gabaix and Laibson, 2002; Reis, 2006a) and price-setting behaviour of firms (Reis, 2006b).

\subsubsection{Costly information acquisition}

As mentioned in the previous Section, information that is particularly valuable for making economic decisions is likely to be costly. In their seminal paper Morris and Shin (2002) solve their model under the assumption of exogenous information quality and free accessibility; and the authors find that in equilibrium individual agents use their information inefficiently. Among other concluding remarks, the authors briefly predict that their main result would still hold and the inefficiency would, in fact, be exacerbated if agents were assumed to engage in costly information acquisition. A similarly brief comment is made by Angeletos and Pavan (2007a) who suggest that "inefficiencies in the use of information are likely to interact with inefficiencies in the collection or aggregation of information" (Angeletos and Pavan, 2007a, p. 1130, italics in original). Hellwig and Veldkamp (2009) provide a formal confirmation of the above predictions in a model that incorporates the Morris-Shin model as a special case, as outlined below.

The model analysed by Hellwig and Veldkamp (2009) is a general one and is formulated so as to impose little restriction on the potential information structure. Economic agents have an option of acquiring a subset of noisy signals about the fundamentals, where the full set consists of signals that can be purely private, purely public or neither ${ }^{25}$. The observed signals are used to inform agents' decisions about actions which are either strategic complements or strategic substitutes, depending on parameter values of the model. The authors find that strategic incentives in information acquisition mirror the strategic motives in action choice (Hellwig and Veldkamp, 2009, p. 229). Thus, an expanded Morris-Shin-type model with endogenous information should exhibit complementarity in both individual actions and individual information acquisition. In other words, from the point of view of an economic agent, additional information becomes more valuable as the amount of aggregate information acquired by other agents increases.

\footnotetext{
${ }^{25}$ Each signal includes an idiosyncratic noise and a common noise; and the coefficients on these noises determine where each signal is located on the public-private spectrum.
} 
In Hellwig and Veldkamp (2009), individual agents decide whether or not to acquire each signal from a given set of signals, that is common across agents. Colombo and Femminis (2008) use a different approach to modelling information endogeneity within the Morris-Shin framework. Maintaining the original assumption that each agent can observe a private and a public signal, the authors allow agents to choose the accuracy of their private information through purchasing units of precision ${ }^{26}$. Public information is also assumed to be endogenous, as the public authorities choose and announce the accuracy of the public signal prior to any decisions being made in the private sector. Colombo and Femminis find that more accurate public information is welfare-enhancing when the marginal cost of acquiring it is lower than that of private information. In fact, the precisions of private and public information are shown to be strategic substitutes. Thus, in equilibrium, positive ${ }^{27}$ precision of public information corresponds to completely uninformative private information, and vice versa. In a way analogous to Angeletos and Pavan (2007a), Colombo et al. (2014) discuss the link between inefficiency in private information acquisition and degrees of coordination in a broad class of models with strategic complementarity or substitutability. For the subclass of models that includes the one used by Colombo and Femminis (2008), the authors find that economic agents underinvest in private information when the equilibrium degree of coordination exceeds the socially optimal one and overinvest when the opposite is true.

Myatt and Wallace (2012) use yet another approach to modelling information acquisition by allowing individual agents to decide how much costly attention to pay to each information source from a given set of information sources. An individual agent observes a private signal from each information source and the overall precision of every observed signal depends on three characteristics: (i) accuracy, (ii) clarity and (iii) attention. Accuracy refers to the quality of the information originating from a given source, clarity characterises an individual agent's imperfect ability to interpret the information from a given source, and attention enhances an agent's ability to interpret each signal due to the effort put into its interpretation. The model does not include exogenously given public signals and instead, in a manner similar to that in Hellwig and Veldkamp (2009), allows private signals to become endogenously public as the amount of aggregate attention paid to them by the private sector increases. The authors demonstrate that economic agents favour the information that is the easiest to interpret, rather than basing their decision on the underlying accuracy of information. Myatt and Wallace also point out that strategic complementarity in actions creates an incentive for the agents to focus on a narrow set of information

\footnotetext{
${ }^{26}$ The term precision refers to the inverse of noise variance.

${ }^{27}$ Here positive refers to an optimally derived finite value defined by model parameters.
} 
sources thereby increasing the degree of publicity of corresponding signals - a result that agrees with the findings by Hellwig and Veldkamp (2009).

Along with costly private information, the above-mentioned literature includes treatment of endogenous acquisition of public information either by the central bank or by the private sector in the form of partially public signals. Arato et al. (2014) set aside considerations of endogeneity of private information and focus on the case where individual agents are able to choose whether or not to purchase a single public signal from the public authorities. The authors discuss the attainability of partial degrees of publicity ${ }^{28}$ and find that, while a fixed price regime yields multiple equilibria, an increasing price rule allows the policymaker to achieve any level of publicity that it deems optimal. The key feature of the suggested pricing rule is that it introduces a substitution effect to offset the complementarity in information acquisition identified in earlier literature.

\subsubsection{Signal value of market aggregates}

In a setting with asymmetric and heterogeneous information, individual agents and policymakers posses valuable private information about the state of economic fundamentals. Such information can be revealed through direct communication or inferred from actions indirectly. The former is within the purview of a central authority that can effectively communicate its private information to the public. In the latter case, information is revealed ex post once an action or actions are observed. A policymaker's private information can be inferred from a policy action taken based on such information. Diverse information possessed by individual agents is reflected in the multitude of strategic market interactions, which, when aggregated "reveal the collective wisdom of all agents in the economy" (Morris and Shin, 2005, p. 15). In Hayek's terms, in an economy with "dispersed bits of incomplete and frequently contradictory knowledge which all the separate individuals possess" (Hayek, 1945, p. 519) market prices become a mechanism for communicating information. In other words, aggregates of market actions (such as prices) possess a signal value for any agent observing said market. One such agent that is interested in observing the market is the central bank.

In discussing the dual role of a central bank in the conduct of monetary policy, Morris and Shin point out that the central bank is both "an active shaper of events"

\footnotetext{
${ }^{28}$ Cornand and Heinemann (2008) analyse a model with an exogenously given fraction of public information users and point out that, should partial announcement policy be optimal, it might be difficult to achieve in practice. Arato et al. (2014) attempt to address this issue through endogeneity.
} 
as well as "a vigilant observer of events" in the economy (Morris and Shin, 2005, p. 3). The two capacities are complementary and contradictory at the same time. On the one hand, closely following the economy allows the central bank to make wellinformed decisions about how to steer it. On the other hand, arguably, the more the central bank shapes the market events, the more such events reflect monetary policy and not underlying fundamentals. The "central bank cannot manipulate prices, and, at the same time, hope the prices yield informative signals" (Morris and Shin, 2005 , p. 3). Informative in this case refers to the informational value in terms of how much of the heterogeneous private information is revealed to the policymaker through the market aggregate. Morris and Shin (2018) label this interaction of the dual role of central banking and market prices as the reflection problem ${ }^{29}$.

The reflection problem arises through two distinct channels. Firstly, as mentioned above, economic outcomes are partly the result of direct monetary policy intervention. Thus, market aggregates partly reflect policy instrument adjustments, which are, in turn, based on information about market aggregates. This channel is generated through direct interaction between monetary policy and the market, and the reflection problem arises independently of private-sector expectations. Secondly, the market understands that monetary policy decisions are based on information revealed to the central bank through observing the market. When it is common knowledge that the central bank is watching the market in order to decide upon its policy stance, this brings about strategic interactions among market participants (Morris and Shin, 2005). If monetary policy is informed by market movements, in order to be able to anticipate policy actions, individual agents aim to anticipate the aggregate of their own actions. Prioritising the strategic motive of second-guessing the market movements over responding to changes in fundamentals inevitably affects the informational content of market movements ${ }^{30}$. In other words, market aggregates partly reflect the awareness of market participants that the aggregate of their own actions has signal value. Both reflection mechanisms demonstrate that the quality of central bank's information is, in fact, endogenous to the economic system the bank operates in (Morris and Shin, 2005).

We organise the relevant literature selected for this brief review around two focal topics: (i) monetary policy design, and (ii) central bank transparency. Both are discussed in relation to signal value of market aggregates.

\footnotetext{
${ }^{29}$ The term reflection problem is a reference to Paul Samuelson's comments on the inadvisability of paying excessive attention to the bond markets, the movements in which can be interpreted merely as a reflection of the central bank's own actions (Fuhrer, 1994).

${ }^{30}$ Even a weak incentive to respond to the agents' expectation of the aggregate action rather than its expectation of the fundamentals can lead to an informational inefficiency.
} 
We begin with the subject of monetary policy rules. Bernanke and Woodford (1997) present a dynamic model of the economy in which a central bank is targeting private sector forecasts. The latter, in this case, acts as both a signal and a stabilisation target for the central bank. Using the model, the authors show that the more successful the bank is at stabilising its target, the less informative the private forecasts become ${ }^{31}$. Thus, Bernanke and Woodford advise against tying monetary policy to "any variable that is too sensitive to the expectations of the public" (Bernanke and Woodford, 1997, p. 656) and instead suggest that private-sector forecast be used in conjunction with other information to inform policy decisions.

Bond and Goldstein (2015) analyse how policy rules affect the signal value of the market aggregate in a model ${ }^{32}$ of direct government intervention into a financial market. The authors establish that price informativeness is endogenous and identify one of the channels of endogeneity as the information importance effect. Namely, the more weight the policymaker puts on the market aggregate, the less important agents' private information becomes in predicting policy actions. As agents' weight on their private information falls, so does the signal value of the aggregate price. This effect is the dominant one for some parameter values ${ }^{33}$, in which case it is advisable for the policymaker to limit its reliance on the market prices as a source of information. The information importance effect is central to the discussion in Morris and Shin (2018) as well as in Chapter 4; however, in this thesis we follow Morris and Shin in using the term reflection problem to refer to the same effect.

Morris and Shin (2018) examine the reflection mechanism in the context of an ad hoc model with endogenously arising strategic complementarities. Their analysis centres around optimal policy design that incorporates policy reacting to a market aggregate. Firstly, the authors find that the policymaker has to take the reflection problem into account, as greater perceived reliance of policy on the market aggregate reduces the signal value of the latter. Secondly, Morris and Shin establish that commitment to a rule that incorporates a response to the market aggregate as well as private information about the state variable produces a superior result compared

\footnotetext{
${ }^{31}$ In the special case, presented in Woodford (1994), in which the market reveals perfect information, and it is optimal for the bank to commit to a policy rule that is inconsistent with a fully informative market signal.

${ }^{32}$ The model does not include an explicit or implicit coordination motive, and its workings and predictions depend on financial risk perception instead.

${ }^{33}$ The second channel is the residual risk effect, which is related to perceived financial risk and works in the opposite direction. The sign of the total effect depends on the source of the risk. Bond and Goldstein distinguish between endogenous risk (related to uncertainty about government actions) and exogenous risk (related to uncertainty about the cash flow from the asset). The strength of the residual risk effect depends on how these sources of risk compare.
} 
to discretionary policy-setting. In particular, the central bank is able to commit to relying less on the market aggregate (compared to the discretionary case) thereby boosting its informativeness.

We now turn to the topic of central bank transparency. In a dynamic coordination scenario with strategic complementarities, Morris and Shin (2005) demonstrate that a central bank that observes market aggregates faces a trade-off between its ability to steer economic agents and the quality of information provided by the market. The central bank learns from the market and publishes its forecast of economic fundamentals, which inform private sector decisions. Individual agents that are engaged in a beauty-contest game use publicly announced forecasts as a coordination tool and place less weight on their private information about the fundamentals. As a result, market aggregates becomes less representative of any new information, and, consequently, the signal value of market aggregates is reduced. Morris and Shin show that the choice of a transparency regime through the mechanism of reflection determines the quality of information available to the central bank.

Similarly to Morris and Shin (2005), Amador and Weill (2010) emphasise the importance of strategic complementarities in the mechanism that leads to a fall in the informativeness of economic aggregates in the presence of public information. Amador and Weill analyse a micro-founded model of the economy in which dispersed private information is aggregated through publicly observable prices. Households, in turn, use aggregate prices to extract endogenous private and public signals about fundamentals. The model features both strategic complementarity and substitutability in households' actions. The authors find that, when strategic complementarities dominate, release of public information about fundamentals can lead to a reduction in informational content of aggregate prices, as individual agents place less emphasis on their private information. Less informative economic aggregates translate to less accurate private signals extracted, which leads to a further reduction in weight that agents put on their private information, and so on, creating an amplifying effect.

Morris and Shin (2005) and Amador and Weill (2010) discuss central bank transparency in terms of revealing a signal about the fundamentals to individual agents. In the context of their financial model already referenced above, Bond and Goldstein (2015) discuss transparency in its wider sense. Namely, the authors distinguish between revealing information about a variable about which individual agents "have at least some additional information" versus a variable about which agents "know less than the government" or nothing at all (Bond and Goldstein, 2015, p. 2782). 
They find that disclosure of policymaker's information is desirable if such information is not available to individual agents from their private sources, for instance, announcements about policy objectives. If, however, market participants already have access to signals about a given variable, policymaker's transparency in regard to information about said variable is harmful and leads to a fall in signal value of market aggregates.

\subsection{Concluding remarks}

In the present Chapter we discuss a variety of topics relevant to the analysis performed in the subsequent Chapters of this thesis. We begin by providing real-world context for the role of central bank transparency as an element of monetary policy. We give an overview of practical considerations in relation to central bank communication as well as touch upon the subject of desirability of transparency. We lay the groundwork for establishing the main points of contact with the theoretical literature on transparency and the Morris and Shin framework, in particular. We then provide a brief overview of theoretical literature that compares and contrasts the monetary policy regimes of commitment and discretion.

Considerable attention is given to reviewing the social value of information literature. The Morris and Shin framework as well as a number of its extensions serve as a foundation that we build upon in our own analysis. Discussing this class of models in detail allows us to draw parallels between established results within this strand of literature and the findings presented in this thesis.

The aim of this Chapter is to provide background as well as the individual building blocks for the modelling carried out in Chapters 2, 3, and 4 . Chapter 2 relies on our discussion of central bank transparency, monetary policy regimes, the Morris and Shin framework, and sticky information. Chapter 3 combines elements of central bank transparency, instrument adjustment, the Morris and Shin model, and costly information acquisition. Finally, Chapter 4 is concerned with analysing the effect of monetary policy on signal value of market aggregates in the context of a variation on the original Morris and Shin model. 


\section{Chapter 2}

\section{Sticky Information}

\section{$2.1 \quad$ Introduction}

Social value of information literature provides a useful context for analysing the interaction of central bank communication and monetary policy. As discussed in Section 1.4.1, in a seminal paper, Morris and Shin (2002) introduce a simple gametheoretic framework that models the effect of public announcements on the actions of economic agents in a setting with heterogeneous information and strategic complementarity. They demonstrate that in some cases increasing the quality of publicly available information is, in fact, detrimental to social welfare. The present Chapter is concerned with expanding and modifying the original Morris-Shin model to include direct monetary policy intervention, explicit communication policy, and information stickiness. In the context of this variation of the Morris-Shin model, our analysis focuses on characterising socially optimal policy design.

The setup of the Morris-Shin framework is such that individual agents have the incentive to coordinate their actions with those of others, while coordination is not optimal for the market as whole. This leads to inefficient use of information in that market participants place excess importance on public announcements, which are viewed as a good indication of what the average action in the market is going to be. Angeletos and Pavan (2007a) show that inefficiency in the way information is used by the agents arises in models of the Morris-Shin type whenever the equilibrium degree of coordination differs from the socially optimal degree of coordination. James and Lawler (2011, 2012b) modify the Morris-Shin framework by considering a payoff function for which the equilibrium degree of coordination is smaller than is socially optimal. This produces the inverse of the Morris and Shin result, i.e. individual agents place excess weight on their private information. In order to broaden the scope of our analysis, in this Chapter we construct a hybrid payoff function, 
that includes the original Morris-Shin and James and Lawler's alternative payoff functions as special cases. In the scenario presented here the precise relationship between the equilibrium and the socially optimal degrees of coordination ${ }^{1}-$ and, thus, the nature of informational inefficiency - depends on parameter values.

Policy intervention has been explored in the literature as a way of counteracting the informational inefficiency in Morris-Shin type models. In the present Chapter, we follow James and Lawler $(2011,2012 \mathrm{a})$ and employ the direct policy intervention approach. James and Lawler allow for monetary policy that directly enters agents' payoffs by impacting market fundamentals. Crucially, the authors shows that, if the information contained in public announcements, instead of being made available to the public, is used to guide optimally designed policy, then an efficient use of information is achieved. One of the aims of this Chapter is to ascertain if the James and Lawler result extends to the scenario considered here. We further extend the analysis of direct intervention by considering two policy regimes - commitment and discretion. As outlined in Section 1.3, we distinguish between the two regimes by the sequence of events in the game and the information that the central bank reveals about its instrument adjustment.

Morris and Shin's original finding about the undesirability ${ }^{2}$ of increasing the quality of publicly available information has important implications for central bank transparency. If a central bank has control over the degree of transparency it exhibits, then it can choose a level of openness that is socially optimal. It is, therefore, useful to be able to introduce the degree of transparency into the model as a central bank's choice variable. Morris and Shin briefly consider the scenario where the policymaker can vary the quality of public announcements up to some upper limit. James and Lawler (2011) modify the Morris-Shin model by assuming that the central bank publishes a less accurate version of the information it uses for its own decision-making. The difference in information quality of the central bank's private information and published information takes on the role of a transparency tool. This way of modelling the degree of transparency conveniently allows for intermediate levels of disclosure thereby enhancing the analysis of welfare effects.

The final element of our variation of the Morris-Shin framework is the assumption of information stickiness. In a different setting to that of the Morris-Shin model, Hahn (2012) looks at desirability of transparency in a signalling game with a cen-

\footnotetext{
${ }^{1}$ In our discussion below, we refer to the socially optimal degree of coordination as collectively efficient.

${ }^{2}$ It should be noted that the circumstances under which this undesirability arises are defined in terms of a set of parameter combinations.
} 
tral bank that discloses its information to price-setting firms. The focus of Hahn's discussion is on the signalling role of policy rather than the coordination motive of individual agents; however, the author makes a useful modelling choice in relation to the information structure of the model. Namely, Hahn adopts the notion of sticky information by assuming that a fraction of firms is oblivious to any new information available in the market. This assumption is not central to Hahn's conclusions, but it is an important building block of the model presented in this Chapter.

In what follows, we first introduce our modelling assumptions in more detail in Section 2.2. We then solve the model under the assumptions of commitment to a rule and discretionary instrument adjustment in Section 2.3 and Section 2.4, respectively. We proceed by comparing how the two regimes perform in terms of expected welfare outcomes and characterise optimal policy design in Section 2.5. Finally, in Section 2.6, we construct and solve an efficient benchmark scenario, which allows us to assess the efficiency of optimally designed policy.

\section{$2.2 \quad$ Model}

In what follows we use a modified version of the original Morris-Shin framework that incorporates selected elements from James and Lawler (2011) and Hahn (2010, 2012). It is a one-shot simultaneous-move game with an infinite number of agents, strategic complementarity, heterogeneous and sticky information, a central bank, and monetary policy ${ }^{3}$. The assumptions of the model and its information structure are set out below.

\subsubsection{Private Sector}

The private sector consists of a continuum of agents that are uniformly distributed over a unit interval and indexed $i \in[0,1]$. It should be noted that here the terms private sector, economy, and market are used interchangeably, hence agents are also referred to as market participants. We assume that within the private sector there are two sources of information asymmetry:

1. sticky information, and

2. heterogeneity of private information.

For now we focus on the former. Following Hahn (2010, 2012), we introduce information stickiness in an ad hoc way and assume that a proportion $Q$ of individual

\footnotetext{
${ }^{3}$ As the original Morris-Shin model, the game presented here is an example of a global game in application. For a concise overview of the global games literature, see Morris (2008).
} 
agents do not respond to any new information. Thus, we distinguish between two types of agents - passive and active ${ }^{4}$. Without loss of generality we order agents so that passive agents correspond to $i \in[0, Q]$ and active agents are distributed over $i \in(Q, 1]$. The parameter $Q$ is exogenous and reflects the degree of information stickiness in a given market ${ }^{5}$.

Before we can further characterise the distinction between passive and active agents, we need to discuss the key assumptions that underlie the strategic behaviour in our model. In particular, we focus on the agents' desire to coordinate their actions with one another.

\subsubsection{Coordination in equilibrium, and efficiency}

As already mentioned in Chapter 1, coordination serves as a central feature in this vein of literature. In fact, it is apparent from the analysis throughout this Chapter, that coordination drives the results in our model. The way in which we discuss coordination heavily relies on the approach described in Angeletos and Pavan (2007a). Naturally, agents' desire to coordinate their actions is reflected in the formulation of their payoff functions.

We assume that individual agents aim to maximise their respective payoffs, which depend on agents' own actions, the action profile of the population, policy instrument adjustment, and the realisation of some state variable $\theta$. The latter reflects market fundamentals and is assumed to be drawn from a uniform distribution over the real line. Agent $i$ 's payoff can then be defined as:

$$
u_{i}=U\left(a_{i}, a_{-i}, \theta, g\right),
$$

where $a_{i}$ is agent $i$ 's own action, $a_{-i} \equiv\left(a_{j}\right)_{j \neq i}$ is the vector of other agents' actions, and $g$ is the monetary policy instrument adjustment. For the purpose of tractability, function $U$ is restricted to being quadratic, which ensures linearity of solutions. Notably, agent $i$ 's payoff depends on actions of other agents $a_{-i}$, and the precise nature of this dependence constitutes the coordination motive.

To further characterise function $U$, we note that the discussion of the Morris and Shin model (Morris and Shin, 2002) in Section 1.4.1 applies more widely here. Namely, we assume that coordination in a model that employs (2.1) as the payoff

\footnotetext{
${ }^{4}$ Active and passive agents correspond to attentive and inattentive agents in the original contribution by Mankiw and Reis (2002) and later in Hahn (2010, 2012).

${ }^{5}$ It should be noted that, due to a difference in definitions of information stickiness, $Q$ corresponds to $1-\lambda$ in Mankiw and Reis (2002) and Hahn $(2010,2012)$.
} 
function stems from strategic complementarity of agents' actions, in the sense of Bulow et al. (1985). Thus, in (2.1), we assume that actions $a_{i}$ and $a_{j}$-where $i \neq j$ - are strategic complements, or, in other words, that the marginal payoff of agent $i$ with respect to $i$ 's action is positively affected by changes in agent $j$ 's action:

$$
\frac{\partial u_{i}}{\partial a_{i} \partial a_{j}}>0
$$

Strategic complementarity in a model like the one used here translates into an incentive to align actions. It should be noted, that Angeletos and Pavan (2007a) extend their analysis to also include strategic substitutability in agents' actions; however, we focus solely on complementarity due to the types of payoff functions used in this thesis. The rationale for using payoff functions with strategic complementarity is explored in more detail in the following Section.

Having formally established the presence of coordination in the model, we now turn to outlining ways to: (i) measure the strength of coordination, as well as (ii) qualify its desirability. In order to accomplish the former task, we need to formally define the equilibrium degree of coordination (or EDoC). Angeletos and Pavan define the $\mathrm{EDoC}$ as the 'private value that agents assign to aligning their choices with those of others' in equilibrium, which in formal terms translates to the 'slope of the best response with respect to aggregate activity' (Angeletos and Pavan, 2007a, p. 1105). Suppose $a_{i}^{*}$ is agent $i$ 's best response that maximises agent $i$ 's payoff, thus, solving:

$$
\frac{\partial u_{i}}{\partial a_{i}}=0
$$

then the EDoC is defined as ${ }^{6}$ :

$$
\mathrm{EDoC} \equiv \frac{\partial a_{i}^{*}}{\partial \bar{a}}
$$

where $\bar{a}$ is the average action profile of the population:

$$
\bar{a} \equiv \int_{0}^{1} a_{j} d j .
$$

A higher value of the EDoC, in (2.3), corresponds to a stronger motive to coordinate. In a symmetric equilibrium, the EDoC is the same for all agents ${ }^{7}$.

At this point, it is important to link coordination and private value of informa-

\footnotetext{
${ }^{6}$ Angeletos and Pavan define the EDoC in more general terms as being equal to $-\frac{\partial u_{i}}{\partial a_{i} \partial \bar{a}} \div$ $\frac{\partial u_{i}}{\partial a_{i} \partial a_{i}}$ (Angeletos and Pavan, 2007a, p. 1112).

${ }^{7}$ For now, we set aside the distinction between active and passive agents.
} 
tion in equilibrium. The model being a one-shot simultaneous-move non-cooperative game implies that agents are unable to achieve coordination through communication with each other or through observing each other's actions prior to taking their own actions. Under these assumptions, agents have no choice, but to achieve alignment of actions through forming expectations about the action profile of the population. Expectations are formed using the private and public information available to each agent; thus, agent $i$ 's best response $a_{i}^{*}$ can be interpreted in terms of how much importance agent $i$ gives to different types of information. Henceforth, we refer to this interpretation as the equilibrium use of information. In a symmetric equilibrium, equilibrium use of information is the same for all agents. Angeletos and Pavan point out that the EDoC conveniently summarises how the coordination motive impacts the equilibrium use of information. An increase in the EDoC implies that agents place more importance on public information, as it is common knowledge and a better predictor of other agents' actions. At the same time, an increase in the EDoC implies that less importance is placed on private heterogeneous information, the latter being associated with differentiation or misalignment of actions.

The EDoC on its own merely allows us to quantify coordination in equilibrium. In order to be able to comment on the desirability of coordination from the point of view of the economy as a whole, we need to define additional measures. Angeletos and Pavan approach this task by introducing a hypothetical scenario referred to as the efficient benchmark, in which the amount of coordination is, in fact, welfaremaximising at the level of the economy. They describe this benchmark case as 'the best society can attain maintaining information decentralized' (Angeletos and Pavan, 2007a, p. 1104). There are three assumptions that are essential to constructing this benchmark scenario. Firstly, the above-mentioned assumption of decentralised information means that we are maintaining the information structure of the model unchanged: agents still have access to heterogeneous private information that they are unable to share with one another and public information that is common knowledge. Agents are still playing a simultaneous-move non-cooperative game, which means they cannot communicate with each other. Secondly, in order to characterise welfare-maximising behaviour, we need to define social welfare. In our model, we define social welfare simply as the aggregate of agents' individual payoffs in (2.1):

$$
W \equiv \frac{1}{N} \int_{0}^{1} u_{i} d i
$$

where $N$ is the normalising factor. Thirdly, we assume that agents are instructed or forced to act in a welfare-maximising way, which may not be individually payoff- 
maximising ${ }^{8}$. This assumption ensures that society is able to attain the best outcome without communication across agents. One possible interpretation is that of central planning $^{9}$ : welfare-maximising decisions are made at a central level and then fed back to individual agents in the form of instructions, which agents then adhere to. By solving the model under the above assumptions we are able to characterise the efficient or first-best outcome, which we are then able to compare with the equilibrium outcome. Importantly, such comparison provides insights into whether any intervention by a policymaker can bring the economy closer to efficiency. In this Chapter we derive the efficient benchmark case in Section 2.6.

In the context of the benchmark scenario described above, we can define a formal efficiency measure for coordination. Collectively efficient degree of coordination ${ }^{10}$ (or $C E D_{o} C$ ) reflects how much society - as opposed to an individual agent - values alignment of individual actions, or, in other words, it captures the desirability of coordination from the point of view of the economy as a whole. Formally, the $\mathrm{CEDoC}$ is the slope of individual agent's best response with respect to aggregate activity, where the best response is derived under the assumptions of the efficient benchmark. The best response in this case maximises ex ante social welfare. Thus, suppose agent $i$ 's welfare-maximising or efficient action $\tilde{a}_{i}$ solves:

$$
\frac{\partial E[W]}{\partial a_{i}}=0
$$

where $E[\cdot]$ denotes unconditional expectations and $W$ is given by (2.5), then CEDoC is defined as $^{11}$ :

$$
\mathrm{CEDoC} \equiv \frac{\partial \tilde{a}_{i}}{\partial \bar{a}}
$$

When the CEDoC is positive, higher values of this measure correspond to greater benefit of coordination to the society.

Similarly to the equilibrium setting, there is an important connection between

\footnotetext{
${ }^{8}$ Angeletos and Pavan offer than alternative hypothetical interpretation of this assumption. Instead of essentially instructing agents to ignore their payoffs, the latter are assumed to be successfully manipulated in a way that ensures that agents' payoff-maximising behaviour is also welfare-maximising.

${ }^{9}$ Angeletos and Pavan (2007a) refer to an entity called the planner.

${ }^{10}$ As mentioned in Section 1.4.2, Angeletos and Pavan refer to this measure as socially optimal degree of coordination. We employ a different term in order to be able to clearly distinguish between efficiency expressions derived under the efficient benchmark assumptions, on the one hand, and equilibrium expressions under socially optimal policy regime design, on the other hand. The latter are derived under normal equilibrium assumptions and relate to central bank decision-making described in Section 2.2.2.

${ }^{11}$ Angeletos and Pavan (2007a) provide a more general formal definition and proof thereof in their paper, however, the simplified expression given in (2.7) is sufficient in the context of this thesis.
} 
collectively efficient coordination and social value of information in the efficient benchmark case. Agent $i$ 's efficient action $\tilde{a}_{i}$ can be interpreted as the collectively efficient response to the different types of available information - private and public. A hypothetical central planner instructs individual agents on how much relative importance they should give to public announcements versus their private heterogeneous information. In this thesis, collectively efficient use of information (which for succinctness, we will often elsewhere shorten to simply efficient) describes the most socially desirable way for individual agents to respond to available information. The nature of the link between the CEDoC and the relative social value of private and public information parallels that of the EDoC. Any changes in the CEDoC correspond to changes in the efficient use of information. Namely, when the CEDoC is positive, an increase in the CEDoC implies that agents should place more importance on common public information and less importance on heterogeneous private information, as making such adjustments would facilitate an increase in coordination.

A particularly useful inference can be made from comparing the EDoC and the CEDoC. Angeletos and Pavan suggest that the divergence of the two measures can indicate the presence of potential payoff externalities, which act as a source of inefficiency in the way information is used in the economy. A mismatch between the two measures corresponds to a mismatch between the perceived value of coordination at an individual level and the true social value of coordination. Such a mismatch leads to inefficient behaviour by agents, which ultimately can be detrimental to social welfare. If the EDoC exceeds the $\mathrm{CEDoC}$, an economy is characterised by excess coordination in equilibrium. Inefficiently strong coordination motive translates into excess importance being placed on common public information and insufficient importance given to heterogeneous private information. The opposite is true if the CEDoC exceeds the EDoC. Comparison of the two measures acts as a useful analytical tool for characterising payoff functions as well as evaluating equilibrium outcomes.

Having identified key characteristics of the payoff function in (2.1) and introduced some useful concepts and definitions, we are now in the position to discuss our choice of payoff function for the model in this Chapter.

\subsubsection{Hybrid payoff function}

We assume that agent $i$ 's payoff is described by the following function:

$$
u_{i}=-[1-(1-\lambda) r]\left[a_{i}-(1-\lambda r)(\theta+g)-\lambda r \bar{a}\right]^{2}-(1-\lambda) r\left(L_{i}-\bar{L}\right) .
$$


The expression in (2.8) is a hybrid payoff function that represents a range of payoff functions defined by the parameter $\lambda \in[0,1]$. The two limiting cases with respect to $\lambda$ represent two payoff functions taken from relevant literature. We first discuss the importance of these two special cases, and then turn to describing the characteristics of the hybrid payoff function and the role of parameter $\lambda$, in particular.

With $\lambda=0$, expression (2.8) becomes the modified version of the Morris-Shin payoff function with direct policy intervention (James and Lawler, 2011):

$$
u_{i}=-(1-r)\left(a_{i}-\theta-g\right)^{2}-r\left(L_{i}-\bar{L}\right)
$$

where $a_{i}$ is agent $i$ 's action, $g$ is the monetary policy instrument ${ }^{12}, r$ reflects the strength of the coordination motive and:

$$
\begin{aligned}
L_{i} & =\int_{0}^{1}\left(a_{j}-a_{i}\right)^{2} d j \\
\bar{L} & =\int_{0}^{1} L_{j} d j .
\end{aligned}
$$

The payoff function in (2.9) is a weighted average of two loss components. The first component of the payoff function is the quadratic loss incurred by the agent when the agent's action does not match the realisation of fundamentals, with direct policy intervention taken into account. The second component characterises the coordination motive - also referred to as the beauty-contest motive ${ }^{13}$ or the second-guessing motive - and includes the loss from the average mismatch between agent $i$ 's action and the actions of others $\left(L_{i}\right)$ corrected for the average of average mismatches across the entire population $(\bar{L})$. Henceforth, we will refer to the payoff function in $(2.9)$ as the $M-S$ case.

The original Morris-Shin payoff function (Morris and Shin, 2002) and (2.9), as in James and Lawler (2011), by extension, were constructed with the purpose of illustrating a situation, in which coordination is only perceived to have value at the individual level. Aggregating the beauty-contest component $-r\left(L_{i}-\bar{L}\right)$ across $i \in$ $[0,1]$ yields zero, which implies that coordination has no welfare benefit at the level of the economy as a whole. In fact, social welfare solely depends on the alignment of agents' actions with the fundamentals and policy instrument adjustment:

$$
W_{\lambda=0}=\frac{1}{1-r} \int_{0}^{1} u_{i} d i=-\int_{0}^{1}\left(a_{i}-\theta-g\right)^{2} d i
$$

\footnotetext{
${ }^{12}$ With $g=0$ the expression in (2.9) becomes the payoff function from Morris and Shin (2002).

${ }^{13}$ This is a reference to Keynes's (1936) famous analogy.
} 
where $\frac{1}{1-r}$ is the normalising factor. This neutrality of coordination is achieved through the inclusion of $-\bar{L}$ in individual payoffs, which, from agent $i$ 's point of view, is merely a constant and plays no role in the payoff-maximising decision. The $\bar{L}$-term ensures that the beauty-contest game of second-guessing decisions of others is zero-sum, meaning that the winners gain at the expense of the losers. The zero-sum nature of the coordination externality leads to coordination being socially inefficient (Morris and Shin, 2002).

As discussed in the previous Section, the mismatch between the private and social value of coordination - implied by the payoff function (2.9) and welfare function (2.10), respectively - can be captured through the comparison of equilibrium and collectively efficient degrees of coordination. It can be shown that:

$$
\begin{aligned}
\mathrm{EDoC}_{\lambda=0} & =r, \\
\mathrm{CEDoC}_{\lambda=0} & =0,
\end{aligned}
$$

thus, the EDoC exceeds the CEDoC (James and Lawler, 2011); and individual agents have an incentive to coordinate their actions, while coordination is neutral at the aggregate level.

The M-S payoff function was originally intended to represent the utility of a financial market participant and capture the behaviour that potentially leads to speculative bubbles (Morris and Shin, 2002). By definition, speculative bubbles are characterised by a rise in asset prices that is driven by speculation about the future value of an asset rather than a true change in underlying fundamentals. Thus, the M-S payoff function captures the potential disconnect between a change in fundamentals and the resulting optimal investment action, since such an action also takes into account an estimate of other investors' beliefs. This example provides one possible interpretation of the individual incentive to coordinate actions and, hence, the positive EDoC that characterises the M-S payoff function. By contrast, the presumption that coordination is socially undesirable - captured by the CEDoC being equal to zero - is more $a d$ hoc in nature. In the context of the financial market example, a zero CEDoC implies that speculative behaviour is harmful for the market. This normative view remains an open debate in the relevant literature and is outside of the scope of this thesis. Angeletos and Pavan (2007a) point out that the usefulness of a beauty-contest payoff function lies in it representing a range of economic scenarios in which agents have an individual incentive to over-react to public information. In this sense, the M-S payoff function is a special case of a setting in which market frictions lead to inefficient use of information and corresponding wel- 
fare loss. The added benefit of including the M-S payoff function into our analysis is that it allows us to draw parallels between our results and existing literature.

With $\lambda=1$, expression (2.8) becomes the alternative payoff function introduced in James and Lawler (2011):

$$
u_{i}=-\left[a_{i}-(1-r)(\theta+g)-r \bar{a}\right]^{2} .
$$

The payoff function in (2.13) is a quadratic loss in the weighted average of the distance between agents $i$ 's action and the market fundamentals (after the effect of policy intervention) as well as the distance between agents $i$ 's action and the average action. In this case, the coordination motive is reflected in the presence of the average action $\bar{a}$ in the payoff function. Henceforth, we will refer to the payoff function in (2.13) as the $J$ - $L$ case.

The J-L payoff function (2.13) is formulated to be directly comparable to the M-S payoff (2.9), as explained by James and Lawler (2011). Maximising both payoffs leads to identical expressions for optimal action $a_{i}^{*}$. In other words, agent $i$ 's incentive to align their action with actions of other agents is the same in both cases. While we can draw a parallel between the two payoff functions at the level of individual behaviour, the key difference between the two functions lies at the aggregate level. Aggregating (2.13) across all agents yields the social welfare expression:

$$
W_{\lambda=1}=\frac{1}{1-r} \int_{0}^{1} u_{i} d i=-\frac{1}{1-r} \int_{0}^{1}\left[a_{i}-(1-r)(\theta+g)-r \bar{a}\right]^{2} d i
$$

In contrast to (2.10), the welfare expression that corresponds to the J-L payoff function contains the coordination parameter $r$, which indicates that coordination is no longer neutral at the level of the economy or market as a whole.

The non-neutrality of coordination at the aggregate level in the J-L case has implications for how we characterise the use of information in equilibrium. As in the M-S case, we need to identify the mismatch between the private and social value of coordination implied by the payoff function (2.13) and the social welfare expression (2.10). For this purpose we first derive the corresponding equilibrium and collectively efficient degrees of coordination:

$$
\begin{aligned}
\operatorname{EDoC}_{\lambda=0} & =r, \\
\mathrm{CEDoC}_{\lambda=0} & =(2-r) r,
\end{aligned}
$$


which confirms that the strength of coordination that arises in equilibrium is, in fact, not collectively efficient. Furthermore, given that the coordination parameter $r$ is restricted to being a positive fraction, the EDoC lies strictly below the CEDoC, which indicates that the individual incentive to coordinate is insufficiently strong, from the point of view of social welfare. In terms of information use, this translates to individual agents under-reacting to publicly available information about the fundamentals and over-reacting to their private heterogeneous information, compared to collectively efficient information use.

The purpose of the J-L payoff function is to capture scenarios in which market participants or economic agents have an incentive to try and predict each other's beliefs about the fundamentals and take such forecasts into account when choosing their optimal actions. This is also the case with the M-S payoff function. In the $\mathrm{J}-\mathrm{L}$ case, however, the wedge that second-guessing behaviour drives between actual changes in underlying fundamentals and agents' best responses is only half of the story. In contrast to the M-S case, successful coordination has social value, and one interpretation of the source of this value is the presence of intrinsic structural linkages that characterise the economy or the market (James and Lawler, 2011). In basic terms, coordination of actions across the population is good for the economy as a whole, and there is coordination taking place in equilibrium, but the strength of equilibrium coordination is insufficiently low.

Although, in the present context, the J-L payoff function represents an abstract strategic setting, it can be linked to specific applications in macroeconomic analysis. Hellwig and Veldkamp (2009) use a utility function that is equivalent to the J-L payoff when discussing information choices in a New Keynesian model with monopolistically competitive firms. The firms are price-setters and adjust their prices taking into account their own forecasts of underlying economic fundamentals as well as the aggregate price. Furthermore, the J-L payoff and welfare functions correspond to a particular case in the micro-founded framework presented in Woodford (2002) and Adam (2007). In this framework, the firms are monopolistically competitive pricesetters, and prices they set exhibit strategic complementarity. The state variable represents the realisation of an aggregate demand or a technology shock that hits the economy. Price or inflation stabilisation is assumed to be one of CB's objectives, and optimal monetary policy is shown to depend on the degree of strategic complementarity. It is important to note that the above is only one possible economic interpretation that is relevant to the J-L payoff function, however, this particular example aptly demonstrates the potential usefulness of including the J-L payoff in our analysis. 
Having looked at the two limiting cases with respect to values of parameter $\lambda$, we now turn our attention back to the hybrid payoff function. The payoff function in (2.8) displays strategic complementarities in the actions of individual agents. Actions $a_{i}$ and $a_{j}$ - where $i \neq j$ - are strategic complements, because the marginal payoff of agent $i$ with respect to $i$ 's action is positively affected by changes in agent j's action:

$$
\frac{\partial u_{i}}{\partial a_{i} \partial a_{j}}=2 r[1-(1-\lambda) \lambda r]>0,
$$

for $0<r<1$ and $0 \leq \lambda \leq 1$. Although strategic complementarity is present for all values of $\lambda$, the strength of the coordination motive and the way agents prioritise different sources of information varies with $\lambda$. For $\lambda$ below unity, incentive to coordinate actions is at least partly caused by the beauty-contest term $-(1-\lambda) r\left(L_{i}-\bar{L}\right)$; and, for non-zero lambda, coordination at least party stems from the presence of the average-action term $-\lambda r \bar{a}$ inside the squared component.

The desirability of coordination also varies with $\lambda$. To demonstrate this, we calculate the equilibrium and collectively efficient degrees of coordination for the payoff function in (2.8), which are:

$$
E D o C=[1-(1-\lambda) \lambda r] r
$$

and

$$
C E D o C=\lambda r(2-\lambda r),
$$

respectively ${ }^{14}$. Figure 2.1 shows the general shape of the curves that represent the two degrees of coordination as functions of $r$. The relationship between the two degrees of coordination is ambiguous and depends on the parameter $\lambda$. The equilibrium degree of coordination exceeds the collectively efficient degree of coordination for $\lambda \in\left[0, \frac{1}{2}\right)$. In other words, when $\lambda<\frac{1}{2}$, the payoff function induces excess coordination, which, in turn, leads to inefficient use of information on behalf of the private sector. As will be shown below, in this case the decisions of individual agents are disproportionately influenced by public announcements. The opposite is true for payoff functions defined by $\lambda \in\left(\frac{1}{2}, 1\right]$, in that agents do not coordinate enough and place inefficiently low importance on public information. There are no informational inefficiencies when $\lambda=\frac{1}{2}$, which corresponds to the equality of the two degrees of coordination $^{15}$. In fact, the absence of informational inefficiencies at $\lambda=\frac{1}{2}$ is the

\footnotetext{
${ }^{14}$ See Appendix B.1 for the derivation of equilibrium and collectively efficient degrees of coordination.

${ }^{15} E D o C$ and $C E D o C$ are also equal when $\lambda=\frac{1}{r}$, however, this value of $\lambda$ eliminates the fundamentals-related component from the payoff function and, thus, is irrelevant to our discussion.
} 
guiding principle behind the formulation of our hybrid payoff function in (2.8). The hybrid payoff function offers generality by incorporating a spectrum of informational inefficiencies.

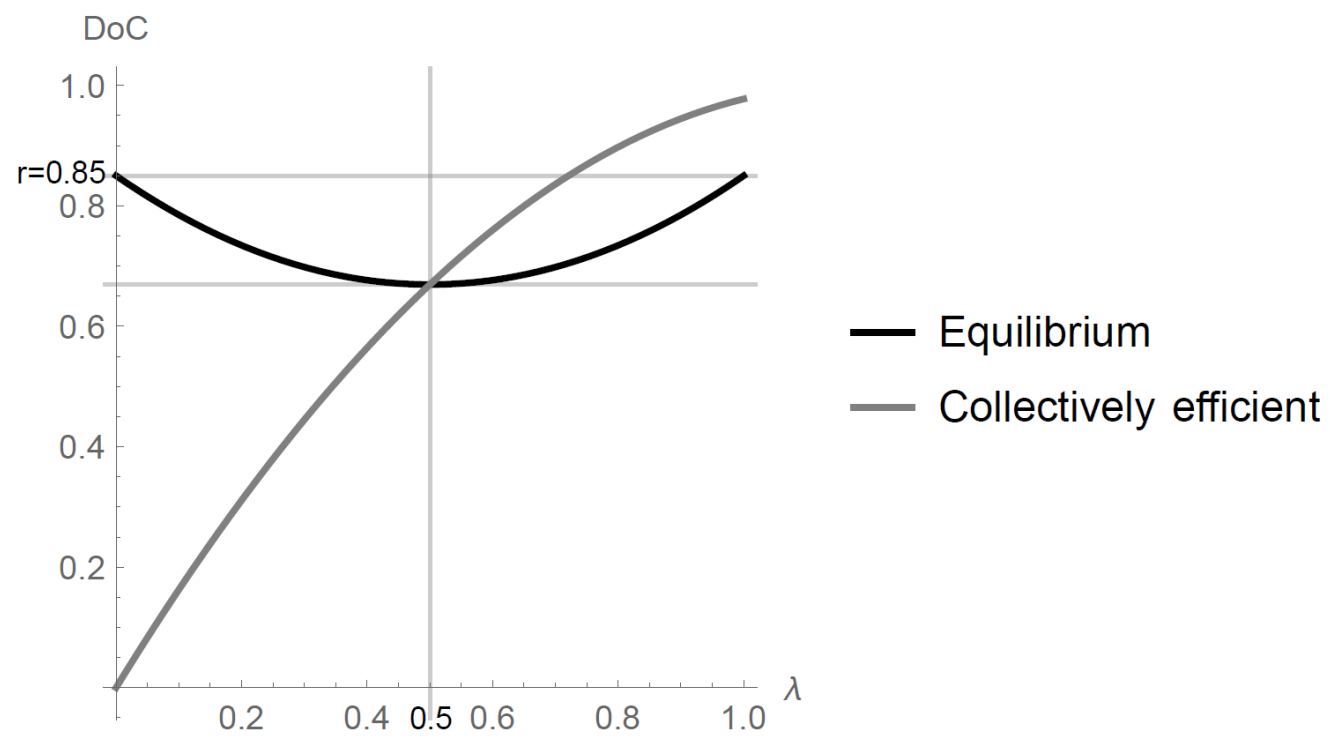

Figure 2.1: Hybrid payoff function. Degrees of coordination as functions of $\lambda$ for $r=0.85$

\subsubsection{Passive agents}

Due to information stickiness, $Q$ of agents are passive in that they ignore all new information information. Passive agents set their action based on the information that is common knowledge before new information becomes available. Thus, agent $i$ 's action is set to be equal to the unconditional expected value of the state variable $\theta$, i.e. zero:

$$
a_{i}^{P}=E[\theta]=0,
$$

where $i \in[0, Q]$ and superscript $P$ stands for passive. Equation (2.20) shows that the assumption we make about the distribution of the state variable $\theta$ leads to these agents not acting at all. Hence, we refer to uninformed agents as passive, rather than inattentive, the latter being the term used in the sticky information literature.

Given that a passive agent's action is zero, agent $i$ 's payoff function defined in (2.8) becomes:

$$
u_{i}^{P}=-[1-(1-\lambda) r][(1-\lambda r)(\theta+g)+\lambda r \bar{a}]^{2}-(1-\lambda) r\left(L_{i}^{P}-\bar{L}\right)
$$

See Appendix B.1. 
where $i \in[0, Q]$ and:

$$
L_{i}^{P}=\int_{0}^{1}\left(a_{j}-a_{i}^{P}\right)^{2} d j .
$$

In principle, subscript $i$ can be dropped in equations (2.20) and (2.21), as there is no heterogeneity in actions or payoffs among the passive agents. However, we retain the subscript for convenience and consistency in notation.

\subsubsection{Active agents}

The remaining $(1-Q)$ of agents have access to two types of information. Firstly, every active agent $i$ observes a noisy private signal $x_{i}$ :

$$
x_{i}=\theta+\varepsilon_{i},
$$

where $i \in(Q, 1], \varepsilon_{i}$ is a normally distributed noise term with a zero mean and some positive variance $\sigma_{\varepsilon}^{2}$. The noise term $\varepsilon_{i}$ is independent of the state variable $\theta$ and the noise terms in other agents' private signals ${ }^{16}$, i.e. $E\left[\varepsilon_{i} \varepsilon_{j}\right]=0$ for $i \neq j$. The private signal $x_{i}$ is the second source of information asymmetry within the private sector. Heterogeneity of private information is due to the noise $\varepsilon_{i}$ being specific to each active agent and agents not being able to observe each others private signals. Secondly, every active agents observes a common public signal $y$, the quality of which is determined by the public authorities ${ }^{17}$. The public signal is the source of commonality of information among active agents and, thus, is relevant to coordination.

The payoff of active agents is given by:

$$
u_{i}^{A}=-[1-(1-\lambda) r]\left[a_{i}^{A}-(1-\lambda r)(\theta+g)-\lambda r \bar{a}\right]^{2}-(1-\lambda) r\left(L_{i}^{A}-\bar{L}\right),
$$

where $i \in(Q, 1]$, superscript $A$ stands for active and:

$$
L_{i}^{A}=\int_{0}^{1}\left(a_{j}-a_{i}^{A}\right)^{2} d j
$$

\footnotetext{
${ }^{16}$ This is a common assumption in this strand of literature - including Morris and Shin (2002) - as it improves the model's tractability and allows the analysis to focus on other variables and assumptions. The Appendix to Morris and Shin (2002) presents a more general case of the original Morris-Shin model that allows for correlated private signals. Morris and Shin show that correlation creates an additional trade-off between the accuracy of a signal and its ability to improve coordination, thereby weakening (yet not fully offsetting) the incentive to underweight the private signal relative to the public signal. By extension, the introduction of correlated noise into our model would have similar implications for information use in equilibrium. The precise effect on collective efficiency of equilibrium outcomes would depend on which payoff is aggregated to produce expected welfare. While assuming $E\left[\varepsilon_{i} \varepsilon_{j}\right] \neq 0$ for $i \neq j$ would complicate the strength of inefficiency, it would not be expected to overturn the general character of the results.

${ }^{17}$ See Section 2.2.2.1 for a definition of $y$.
} 
Given definitions of $L_{i}^{P}$ and $L_{i}^{A}, \bar{L}$ can be re-defined as:

$$
\bar{L}=\int_{0}^{Q} L_{j}^{P} d j+\int_{Q}^{1} L_{j}^{A} d j .
$$

Active agents choose their actions so as to maximise their payoffs. Differentiating (2.23) with respect to $a_{i}^{A}$, solving the resulting first-order condition and taking expectations of the solution yields:

$$
a_{i}^{A}=\left[1-r+(1-\lambda) \lambda r^{2}\right]\left(E_{i}[\theta]+E_{i}[g]\right)+r[1-(1-\lambda) \lambda r] E_{i}[\bar{a}]
$$

where $E_{i}[\cdot]$ denotes expectations conditional on agent $i$ 's information set. The presence of average action $\bar{a}$ in the expression for optimal action reflects the influence of the coordination motive. Following Morris and Shin (2002), we assume that the optimal strategy can be expressed as a linear function of the observed signals:

$$
a_{i}^{A}=\kappa_{0}+\kappa_{1} x_{i}+\kappa_{2} y
$$

where $\kappa_{0}, \kappa_{1}$ and $\kappa_{2}$ are optimal weights. These optimal weights characterise equilibrium use of information.

\subsubsection{Population action profile}

Given information stickiness, the expression for average action in (2.4) becomes:

$$
\bar{a}=\int_{0}^{Q} a_{i}^{P} d i+\int_{Q}^{1} a_{i}^{A} d i .
$$

Substituting optimal actions for active and passive agents (2.20) and (2.25), respectively, as well as the definition of $x_{i}$ into the above expression for average action and integrating yields:

$$
\begin{aligned}
& \bar{a}=\int_{0}^{Q} 0 d i+\int_{Q}^{1}\left(\kappa_{0}+\kappa_{1} x_{i}+\kappa_{2} y\right) d i \\
& \bar{a}=(1-Q)\left[\kappa_{0}+\kappa_{1} \int_{Q}^{1}\left(\theta+\varepsilon_{i}\right) d i+\kappa_{2} y\right] \\
& \bar{a}=(1-Q)\left(\kappa_{0}+\kappa_{1} \theta+\kappa_{2} y\right),
\end{aligned}
$$

where we used the fact that $\int_{Q}^{1} \varepsilon_{i} d i=0$ and that $y$ is common knowledge among active agents. 


\subsubsection{Social welfare}

In this model the expression for social welfare is obtained simply by aggregating individual payoffs across the population. Thus, the normalised average of individual utilities from (2.8) is given by:

$$
\begin{aligned}
& W=\frac{1}{1-(1-\lambda) r}\left\{\int_{0}^{Q} u^{P} d i+\int_{Q}^{1} u_{i}^{A} d i\right\} \\
& W=-Q[(1-\lambda r)(\theta+g)+\lambda r \bar{a}]^{2}-\int_{Q}^{1}\left[a_{i}^{A}-(1-\lambda r)(\theta+g)-\lambda r \bar{a}\right]^{2} d i .
\end{aligned}
$$

The welfare expression of equation (2.28) is the ex post welfare that depends on the realisation of the state variable $\theta$, the policy instrument $g$ and actions of active agents. Taking expectations of (2.28) yields the ex ante welfare (see Appendix B.2) used for assessing the expected welfare outcome.

\subsubsection{Central Bank}

One other agent in the model is a benevolent central bank (CB) that aims to maximise social welfare by making optimal decisions about communication and monetary policy. The term benevolence here refers to the absence of a separate objective function for the CB that could potentially reflect CB's own incentives (Walsh (1995)) or conservatism (Rogoff (1985)). The CB is assumed to disregard any additional consideration and focus on minimising the social loss. It should be noted, that here the terms $C B$, policymaker, monetary and public authorities are used interchangeably. In our assumptions about the CB's information set and decision-making we closely follow James and Lawler (2011).

\subsubsection{Central bank communication}

There is asymmetric information between the $\mathrm{CB}$ and the private sector, as the former cannot observe agents' private signals. There is potential for another source of informational asymmetry, as the CB receives a noisy private signal that is not observed by other market participants in its original form. This private signal is defined as:

$$
z=\theta+\phi
$$

where $\phi$ is a normally distributed noise term with a zero mean and some positive variance $\sigma_{\phi}^{2}$. The noise $\phi$ is independent of the state variable $\theta$ and private sector noise terms $\varepsilon_{i}$, where $i \in(Q, 1]$. We do not make any additional assumptions about the relative informedness of the $\mathrm{CB}$ and the private sector, as measured by the two noise variances $\sigma_{\phi}^{2}$ and $\sigma_{\varepsilon}^{2}$. In his comment on the original Morris-Shin result, 
Svensson (2006) argues that it is reasonable to assume that the policymaker is at least as informed as the market participants, with the conservative benchmark being $\sigma_{\phi}^{2}=\sigma_{\varepsilon}^{2}$. Svensson refers to empirical evidence presented by Romer and Romer (2000), who find that the U.S. Federal Reserve appears to have had an informational advantage over commercial forecasters over the period 1965-1991. D'Agostino and Whelan (2008) repeat the exercise for the period 1974-2001 and find that in the last decade of the sample period the Federal Reserve has, in fact, lost its forecasting advantage $^{18}$. Thus, it would seem to be unjustified to impose any restrictions on the relative values of $\sigma_{\phi}^{2}$ and $\sigma_{\varepsilon}^{2}$.

Having received the private signal $z$, the bank makes a public announcement which is a noisy version of its private signal:

$$
y=z+\eta=\theta+\phi+\eta
$$

where $\eta$ is a normally distributed noise term with a zero mean and some variance $\sigma_{\eta}^{2}$. The noise $\eta$ is independent of $\theta, \phi$ and $\varepsilon_{i}$, where $i \in(Q, 1]$. The variance of the additional noise $\sigma_{\eta}^{2}$ acts as a transparency tool, that can be optimally chosen by the CB to maximise social welfare:

- $\sigma_{\eta}^{2}=0$ corresponds to full transparency, as $y$ collapses to $z$;

- finite positive values of $0<\sigma_{\eta}^{2}$ correspond to intermediate degrees of transparency;

- $\sigma_{\eta}^{2} \rightarrow \infty$ renders the public signal completely uninformative and characterises full opacity.

Thus, any non-zero values of the additional noise $\eta$ create informational asymmetry between the $\mathrm{CB}$ and the private sector. The ability to adjust $\sigma_{\eta}^{2}$ allows the bank to influence the market outcome indirectly, through influencing the behaviour of individual agents. For instance, lowering the precision of the public signal (by increasing the variance of the additional noise) would encourage market participants to rely more heavily on their private information, and vice versa. In other words, adjustments to $\sigma_{\eta}^{2}$ have the potential to change the weights $\kappa_{1}$ and $\kappa_{2}$ in equation $(2.25)$.

\subsubsection{Direct policy intervention}

In addition to choosing the level of transparency, the CB has the ability to influence the market outcome directly by adjusting the policy instrument $g$. As can be seen

\footnotetext{
${ }^{18}$ D'Agostino and Whelan (2008) suggest two possible explanations for the increase in accuracy of commercial forecasts: (i) higher predictability of monetary policy, and (ii) increased transparency.
} 
from the payoff function (2.8) and the social welfare function (2.28), the policy instrument is set in a way that would counteract the impact of the state variable $\theta$. The decision on the value of $g$ is made prior to the CB observing the realisation of $\theta$ or agents' actions based on the bank's current information. We assume that the instrument adjustment is a linear function of the policymaker's private information $z$ and the public signal $y$ :

$$
g=\rho_{0}+\rho_{1} z+\rho_{2} y
$$

where $\rho_{0}, \rho_{1}$ and $\rho_{2}$ are policy parameters chosen optimally by the CB.

In the tradition followed by the literature on monetary policy, we consider two policy regimes:

1. commitment to a rule, and

2. discretion.

The difference between the two regimes lies in what market participants know about the setting of monetary policy. When the $\mathrm{CB}$ is committed to a rule, the policy parameters $\rho_{0}, \rho_{1}$ and $\rho_{2}$ are common knowledge from the outset of the game. On the other hand, when the policy is set in a discretionary fashion, individual agents are aware of the fact that the $\mathrm{CB}$ is aiming to maximise social welfare and they know the expression for social welfare (2.28) as well as the general form of the policy function (2.31). Given this information, agents infer the values of the policy parameters $\rho_{0}, \rho_{1}$ and $\rho_{2}$ and incorporate these predictions into their choice of optimal action. The general form of the policy function in (2.31) remains the same in both cases, however, the choice of the policy regime affects the sequence of steps taken in solving the model.

\subsubsection{Private sector expectations}

As noted above, active agents cannot observe the state variable $\theta$ directly and, thus, form expectations of $\theta$ conditional on the observed signals. Agent $i$ 's expectation of $\theta$ is a linear combination of signals $x_{i}$ and $y^{19}$ :

$$
E_{i}[\theta]=\frac{\left(\sigma_{\phi}^{2}+\sigma_{\eta}^{2}\right) x_{i}+\sigma_{\varepsilon}^{2} y}{\sigma_{\varepsilon}^{2}+\sigma_{\phi}^{2}+\sigma_{\eta}^{2}},
$$

where $i \in(Q ; 1]$. Expression (2.32) is a weighted average of signals $x_{i}$ and $y$, where weights inversely depend on the relative noisiness of the two signals and are expressed in terms of noise variances, which are common knowledge. Alternatively, one can

\footnotetext{
${ }^{19}$ See Appendix B.3 for the derivation of the expected value of $\theta$.
} 
say that the weights depend on the relative precision of signals $x_{i}$ and $y$, with more weight put on the more precise signal. Morris and Shin (2002) define signal precision for each signal as the inverse of the respective noise variance ${ }^{20}$ :

$$
\begin{aligned}
& \alpha \equiv \frac{1}{\sigma_{\phi}^{2}+\sigma_{\eta}^{2}} \text { for } \sigma_{\eta}^{2}=0 \\
& \beta \equiv \frac{1}{\sigma_{\varepsilon}^{2}}
\end{aligned}
$$

and subsequently solve and analyse their model in terms of signal precisions. In the present model we need to be able to distinguish between the effects of the two noises $\phi$ and $\eta$, thus one possibility for defining precisions of $x_{i}, z$ and $y$ is:

$$
\begin{aligned}
\alpha & \equiv \frac{1}{\sigma_{\phi}^{2}} \\
\beta & \equiv \frac{1}{\sigma_{\varepsilon}^{2}} \\
\gamma & \equiv \frac{1}{\sigma_{\phi}^{2}+\sigma_{\eta}^{2}}=\frac{\alpha}{1+\alpha \sigma_{\eta}^{2}},
\end{aligned}
$$

respectively. However, we refrain from introducing precisions into our model, as it does not enhance tractability.

Unless the CB exercises full transparency, active agents cannot directly observe the bank's private signal $z$. Therefore, individual agents use the information they posses to form an estimate of $z$, which is, in turn, used to make predictions about the monetary instrument adjustment $g$. Agent $i$ 's expectation of $z$ is a linear combination of signals $x_{i}$ and $y^{21}$ :

$$
E_{i}[z]=\frac{\sigma_{\eta}^{2} x_{i}+\left(\sigma_{\varepsilon}^{2}+\sigma_{\phi}^{2}\right) y}{\sigma_{\varepsilon}^{2}+\sigma_{\phi}^{2}+\sigma_{\eta}^{2}},
$$

where $i \in(Q, 1]$. By combining the two signals, agents obtain a more accurate estimate of $z$, compared to an estimate formed using just the public signal $y$. Again, expression (2.33) is a weighted average of the two observed signals; and the greater is the variance of the additional noise, the more weight is placed on agent $i$ 's private signal. Under full transparency, the right-hand side of expression (2.33) collapses to $y$, which, in turn, is equal to the actual value of $z$ in the absence of additional noise.

\footnotetext{
${ }^{20}$ Morris and Shin do not distinguish between the policymaker's private signal and the public signal $(z=y)$, thus the definition of $\alpha$ as the precision of the public signal is only consistent with our notation when $\sigma_{\eta}^{2}=0$.

${ }^{21}$ See Appendix B.3 for the derivation of the expected value of $z$.
} 


\subsection{Commitment to a Rule}

In this Section we analyse the model using the assumption that the CB commits to a monetary policy rule. The rule in its general form is described by equation (2.31):

$$
g=\rho_{0}+\rho_{1} z+\rho_{2} y
$$

where policy parameters $\rho_{0}, \rho_{1}$ and $\rho_{2}$ are assumed to be known to the public from the outset. Before we proceed to solving the model in its entirety, we first look at a simplified version of the setup, in which information stickiness is assumed away. The main purpose of this exercise is to establish a firm point of contact with existing literature.

\subsubsection{Equilibrium without passive agents $(Q=0)$}

In the absence of passive agents $(Q=0)$, an active agent $i$ - where $i \in[0,1]-$ observes a private signal $x_{i}$, a public signal $y$ as well as policy parameters $\rho_{0}, \rho_{1}$ and $\rho_{2}$ announced by the CB. We begin by deriving the optimal action of an active agent $i$, taking the policy parameters as given. Agent $i$ 's expectation of the policy instrument adjustment is given by:

$$
E_{i}[g]=\rho_{0}+\rho_{1} E_{i}[z]+\rho_{2} y
$$

where $i \in[0,1]$ and the expectation of $z$, is given by (2.33). Similarly, agent $i$ 's expectation of the average market action is:

$$
E_{i}[\bar{a}]=\kappa_{0}+\kappa_{1} E_{i}[\theta]+\kappa_{2} y
$$

where the expectation of $\theta$ is given by (2.32). From equations (2.24) and (2.25), the two representations of agent $i$ 's optimal action are:

$$
\left\{\begin{array}{l}
a_{i}^{A}=\left[1-r+(1-\lambda) \lambda r^{2}\right]\left(E_{i}[\theta]+E_{i}[g]\right)+r[1-(1-\lambda) \lambda r] E_{i}[\bar{a}] \\
a_{i}^{A}=\kappa_{0}+\kappa_{1} x_{i}+\kappa_{2} y .
\end{array}\right.
$$

We proceed by applying the method of undetermined coefficients to the above expressions. Substituting agent $i$ 's expectation of the state variable $\theta$ from (2.32), the CB's private signal $z$ from (2.33), the average action from (2.35) and the instrument adjustment $g$ from (2.34) into the first of the two expressions for optimal action and comparing the coefficients on $x_{i}$ and $y$ to those in the alternative representation yields a system of equation. Solving the system gives us the following expressions 
for equilibrium weights:

$$
\begin{aligned}
& \kappa_{0}^{*}=\rho_{0} \\
& \kappa_{1}^{*}=\frac{\left[1-r+(1-\lambda) \lambda r^{2}\right]\left[\sigma_{\phi}^{2}+\left(1+\rho_{1}\right) \sigma_{\eta}^{2}\right]}{\sigma_{\varepsilon}^{2}+\left[1-r+(1-\lambda) \lambda r^{2}\right]\left(\sigma_{\phi}^{2}+\sigma_{\eta}^{2}\right)} \\
& \kappa_{2}^{*}=\frac{\left(1+\rho_{1}+\rho_{2}\right) \sigma_{\varepsilon}^{2}+\left[1-r+(1-\lambda) \lambda r^{2}\right]\left[\left(\rho_{1}+\rho_{2}\right) \sigma_{\phi}^{2}+\rho_{2} \sigma_{\eta}^{2}\right]}{\sigma_{\varepsilon}^{2}+\left[1-r+(1-\lambda) \lambda r^{2}\right]\left(\sigma_{\phi}^{2}+\sigma_{\eta}^{2}\right)} .
\end{aligned}
$$

The policymaker knows that the equilibrium actions of market participants are represented by the optimal weights (2.37a)-(2.37c) and aims to maximise expected social welfare, given those weights.

In order to proceed with our analysis we need to derive the expression for $e x$ ante expected social welfare. In the absence of passive agents $(Q=0)$ the welfare expression (2.28) becomes:

$$
\left.W\right|_{Q=0}=\int_{0}^{1}-\left[a_{i}^{A}-(1-\lambda r)(\theta+g)-\lambda r \bar{a}\right]^{2} d i
$$

We then substitute the expressions for optimal action (2.25), average action (2.27), policy function (2.31) as well as the definitions of signals (2.22), (2.29) and (2.30) into the welfare function (2.38) and take expectations of the resulting expression, using the assumptions about the distributions of $\theta, \varepsilon_{i}, \phi$ and $\eta$. The expected welfare function is then given by:

$$
\begin{aligned}
E[W]_{Q=0}= & -\kappa_{1}^{2} \sigma_{\varepsilon}^{2}-(1-\lambda r)^{2}\left[\left(\kappa_{0}-\rho_{0}\right)^{2}+\left(\kappa_{1}+\kappa_{2}-1-\rho_{1}-\rho_{2}\right)^{2} \sigma_{\theta}^{2}+\right. \\
& \left.+\left(\kappa_{2}-\rho_{1}-\rho_{2}\right)^{2} \sigma_{\phi}^{2}+\left(\kappa_{2}-\rho_{2}\right)^{2} \sigma_{\eta}^{2}\right]
\end{aligned}
$$

Having derived expected welfare expression for $Q=0$, we substitute the equilibrium weights (2.37a)-(2.37c) into (2.39) and we obtain:

$$
\begin{aligned}
E[W]_{Q=0}= & -\frac{1}{\Omega_{1}} \cdot\left\{\left[1-r+(1-\lambda) \lambda r^{2}\right]^{2} \sigma_{\varepsilon}^{2}\left[\sigma_{\phi}^{2}+\left(1+\rho_{1}\right) \sigma_{\eta}^{2}\right]^{2}+\right. \\
& +(1-\lambda r)^{2} \sigma_{\phi}^{2}\left[\sigma_{\varepsilon}^{2}+\left(1-r+(1-\lambda) \lambda r^{2}\right) \rho_{1} \sigma_{\eta}^{2}\right]^{2}+ \\
& \left.+(1-\lambda r)^{2} \sigma_{\eta}^{2}\left[\left(1+\rho_{1}\right) \sigma_{\varepsilon}^{2}+\left(1-r+(1-\lambda) \lambda r^{2}\right) \rho_{1} \sigma_{\phi}^{2}\right]^{2}\right\}
\end{aligned}
$$

where $\Omega_{1} \equiv\left\{\sigma_{\varepsilon}^{2}+\left[1-r+(1-\lambda) \lambda r^{2}\right]\left(\sigma_{\phi}^{2}+\sigma_{\eta}^{2}\right)\right\}^{2}$. Expression (2.40) is independent of $\rho_{0}$ and $\rho_{2}$, which implies that the private sector fully offsets the effect of the terms $\rho_{0}$ and $\rho_{2} y$ on welfare. Without loss of generality we assume $\rho_{0}^{*}=\rho_{2}^{*}=\kappa_{0}^{*}=0$. Minimising the expected welfare loss with respect to $\rho_{1}$ yields the following value 
for the optimal policy parameter:

$$
\rho_{1}^{*}=-\frac{\sigma_{\varepsilon}^{2}\left\{\sigma_{\varepsilon}^{2}+[1-(1-\lambda) r]^{2}\left(\sigma_{\phi}^{2}+\sigma_{\eta}^{2}\right)\right\}}{\Omega_{2}},
$$

where:

$$
\begin{aligned}
\Omega_{2} \equiv & \sigma_{\varepsilon}^{4}+[1-(1-\lambda) r] \sigma_{\varepsilon}^{2}\left\{2(1-\lambda r) \sigma_{\phi}^{2}+[1-(1-\lambda) r] \sigma_{\eta}^{2}\right\}+ \\
& +\left[1-r+(1-\lambda) \lambda r^{2}\right]^{2} \sigma_{\phi}^{2}\left(\sigma_{\phi}^{2}+\sigma_{\eta}^{2}\right) .
\end{aligned}
$$

Given that the optimal policy rule is:

$$
g=\rho_{1}^{*} z
$$

the ex ante equilibrium welfare becomes:

$$
E\left[W_{R}\right]_{Q=0}=(1-\lambda r)^{2} \rho_{1}^{*} \sigma_{\phi}^{2}
$$

Expression (2.42) is strictly increasing in $\sigma_{\eta}^{2}$, thus the policy of full opacity is optimal. In the limit, as $\sigma_{\eta}^{2} \rightarrow \infty$, expected welfare approaches the following expression:

$$
E\left[W_{R}^{Z e r o}\right]_{Q=0}=-\frac{(1-\lambda r)^{2} \sigma_{\varepsilon}^{2} \sigma_{\phi}^{2}}{\sigma_{\varepsilon}^{2}+(1-\lambda r)^{2} \sigma_{\phi}^{2}} .
$$

Setting $\lambda$ equal to zero or unity in equation (2.43) yields welfare expressions equivalent to those presented in James and Lawler (2011) and James and Lawler (2012b), respectively. In the following Section we re-introduce the assumption of sticky information and solve the model for $0<Q<1$.

\subsubsection{Equilibrium with passive agents $(0<Q<1)$}

Following the same steps as in the previous Section, we begin by determining the equilibrium actions of active agents, given information stickiness $(0<Q<1)$ and the welfare expression (2.28). In this Section we restrict our analysis to intermediate levels of information stickiness with the proportion of passive agents being above zero and below unity. We discuss the special case where all agents are passive $(Q=1)$ at a later stage.

An active agent $i$ - where $i \in(Q, 1]$ - knows the general form of the policy rule, thus, we substitute:

$$
E_{i}[g]=\rho_{0}+\rho_{1} E_{i}[z]+\rho_{2} y
$$


from (2.34) and agent $i$ 's expectation of the average action from (2.27):

$$
E_{i}[\bar{a}]=(1-Q)\left(\kappa_{0}+\kappa_{1} E_{i}[\theta]+\kappa_{2} y\right),
$$

into the first of the two representations of optimal action:

$$
\left\{\begin{array}{l}
a_{i}^{A}=\left[1-r+(1-\lambda) \lambda r^{2}\right]\left(E_{i}[\theta]+E_{i}[g]\right)+r[1-(1-\lambda) \lambda r] E_{i}[\bar{a}] \\
a_{i}^{A}=\kappa_{0}+\kappa_{1} x_{i}+\kappa_{2} y
\end{array}\right.
$$

from (2.24) and (2.25). The method of undetermined coefficients yields the following expressions for optimal weights:

$$
\begin{aligned}
\kappa_{0}^{*} & =\frac{\left[1-r+(1-\lambda) \lambda r^{2}\right] \rho_{0}}{\Omega_{3}} \\
\kappa_{1}^{*} & =\frac{\left[1-r+(1-\lambda) \lambda r^{2}\right]\left[\sigma_{\phi}^{2}+\left(1+\rho_{1}\right) \sigma_{\eta}^{2}\right]}{\sigma_{\varepsilon}^{2}+\Omega_{3}\left(\sigma_{\phi}^{2}+\sigma_{\eta}^{2}\right)} \\
\kappa_{2}^{*} & =\frac{\left[1-r+(1-\lambda) \lambda r^{2}\right]\left\{\left(1+\rho_{1}+\rho_{2}\right) \sigma_{\varepsilon}^{2}+\Omega_{3}\left[\left(\rho_{1}+\rho_{2}\right) \sigma_{\phi}^{2}+\rho_{2} \sigma_{\eta}^{2}\right]\right\}}{\Omega_{3}\left[\sigma_{\varepsilon}^{2}+\Omega_{3}\left(\sigma_{\phi}^{2}+\sigma_{\eta}^{2}\right)\right]}
\end{aligned}
$$

where $\Omega_{3} \equiv 1-(1-Q) r+(1-\lambda) \lambda(1-Q) r^{2}$.

The policymaker uses the information about the equilibrium behaviour of active agents in conjunction with the ex ante welfare expression to choose the precise shape of the policy rule. Thus, we substitute equilibrium weights $(2.46 \mathrm{a})-(2.46 \mathrm{c})$ into the welfare expression (B.20) in Appendix B.2. To simplify the resulting expression we use the following constraint on the policy parameters:

$$
\rho_{1}+\rho_{2}=-1
$$

from (B.22), which is one of the optimality conditions for welfare-maximisation. As explained in Appendix B.2, this constraint ensures that expected welfare loss takes on a finite value. Having substituted $\rho_{2}=-\left(1+\rho_{1}\right)$ into the expected welfare expression, we minimise it with respect to $\rho_{0}$ and $\rho_{1}$. We find that optimal policy is given by:

$$
g=-z-\left(1+\rho_{1}^{*}\right) \eta
$$

where $\rho_{0}^{*}=0$ and:

$$
\rho_{1}^{*}=-\frac{\sigma_{\varepsilon}^{4}+\sigma_{\varepsilon}^{2}\left(\sigma_{\phi}^{2}+\sigma_{\eta}^{2}\right) \Omega_{4}+Q\left(\sigma_{\phi}^{2}+\sigma_{\eta}^{2}\right)^{2} \Omega_{5}}{\sigma_{\varepsilon}^{4}+\sigma_{\varepsilon}^{2}\left(2 \sigma_{\phi}^{2} \Omega_{3}+\sigma_{\eta}^{2} \Omega_{4}\right)+\left(\sigma_{\phi}^{2}+\sigma_{\eta}^{2}\right)\left(\sigma_{\phi}^{2} \Omega_{3}^{2}+Q \sigma_{\eta}^{2} \Omega_{5}\right)},
$$


where:

$$
\begin{aligned}
& \Omega_{3} \equiv 1-(1-Q) r+(1-\lambda) \lambda(1-Q) r^{2} \\
& \Omega_{4} \equiv[1-(1-\lambda) r]^{2}+Q\left[1+2(1-\lambda) r-(1-\lambda)^{2} r^{2}\right] \\
& \Omega_{5} \equiv 1-2(1-\lambda)(1-Q) r+(1-\lambda)^{2}(1-Q) r^{2} .
\end{aligned}
$$

Given the optimal policy rule (2.47), expected equilibrium welfare can be expressed as:

$$
E\left[W_{R}\right]=(1-\lambda r)^{2} \rho_{1}^{*} \sigma_{\phi}^{2},
$$

which is equivalent to (2.42) if we set $Q=0$.

The CB uses (2.49) to determine the optimal disclosure policy by finding the value of $\sigma_{\eta}^{2}$ that minimises the expected social loss. We are going to split further analysis into three separate cases:

1. the general case of the hybrid payoff function $(0<\lambda<1)$;

2. the M-S payoff function $(\lambda=0)$; and

3. the J-L payoff function $(\lambda=1)$.

We do so to make the results directly comparable with the results in the relevant literature as well as for the convenience of presentation.

\subsubsection{Hybrid payoff function $(0<\lambda<1)$}

The expected equilibrium welfare function in (2.49) is continuous in the variance of the additional noise $\sigma_{\eta}^{2}$, allowing us to use standard optimisation techniques. The first derivative of expected equilibrium welfare with respect to the transparency tool $\sigma_{\eta}^{2}$ has an ambiguous sign. Solving the first order condition yields two stationary points, one of which is always negative and the other is positive for combinations of parameter values described by a union of six sets of conditions. The conditions in question are presented in Appendix B.4 and, when these conditions are met, the stationary point is an optimum.

The expressions for the optimal value of $\sigma_{\eta}^{2}$ under partial transparency and the corresponding level of expected equilibrium welfare are omitted here due to their excessive length. The welfare outcome under full transparency is obtained by setting 
$\sigma_{\eta}^{2}=0$ in $(2.49)$

$E\left[W_{R}^{F u l l}\right]=-\frac{(1-\lambda r)^{2} \sigma_{\phi}^{2}\left(\sigma_{\varepsilon}^{2}+Q \sigma_{\phi}^{2}\right)\left\{\sigma_{\varepsilon}^{2}+[1-(1-\lambda)(1-Q)(2-(1-\lambda) r) r] \sigma_{\phi}^{2}\right\}}{\left\{\sigma_{\varepsilon}^{2}+\left[1-(1-Q) r+(1-\lambda)(1-Q) \lambda r^{2}\right] \sigma_{\phi}^{2}\right\}^{2}}$,

whereas the welfare outcome under zero transparency corresponds to setting $\sigma_{\eta}^{2} \rightarrow$ $\infty$ :

$$
E\left[W_{R}^{Z e r o}\right]=-(1-\lambda r)^{2} \sigma_{\phi}^{2}
$$

Differentiating (2.50) with respect to model parameters reveals that under full transparency expected welfare is always decreasing as the private sector becomes less informed (as $\sigma_{\varepsilon}^{2}$ increases) or as the proportion of passive agents $Q$ increases. The welfare is increasing in the quality of CB's information with the exception of a subset of parameter combinations with low values of $\lambda$, in which case the sign of the derivative is ambiguous ${ }^{22}$. Differentiation of (2.51) shows that under zero disclosure welfare is independent of the degree of information stickiness and the quality of active agents' private signals. In the case of zero transparency, an increase in the quality of CB's information is unambiguously beneficial.

In order to determine, which of the three possible transparency regimes - full, partial or zero - is optimal, we compare the corresponding expected welfare outcomes. The comparison reveals that, when the value of $\sigma_{\eta}^{2}$ corresponding to the stationary point is positive, partial transparency is optimal, otherwise either full or zero transparency is optimal, depending on parameter values ${ }^{23}$. In other words, the precise combination of parameter values dictates whether the CB should adopt the policy of full, partial or zero transparency.

\subsubsection{M-S payoff function $(\lambda=0)$}

Setting $\lambda=0$ in the expected equilibrium welfare expression in (2.49) yields:

$$
E\left[W_{R}\right]_{\lambda=0}=-\frac{\left[\sigma_{\varepsilon}^{2}+Q\left(\sigma_{\phi}^{2}+\sigma_{\eta}^{2}\right)\right]\left[\sigma_{\varepsilon}^{2}+\left(\sigma_{\phi}^{2}+\sigma_{\eta}^{2}\right) \Omega_{6}\right] \sigma_{\phi}^{2}}{\sigma_{\varepsilon}^{4}+\sigma_{\varepsilon}^{2}\left\{2 \sigma_{\phi}^{2} \Omega_{7}+\sigma_{\eta}^{2} \Omega_{8}\right\}+\left(\sigma_{\phi}^{2}+\sigma_{\eta}^{2}\right)\left\{\sigma_{\phi}^{2} \Omega_{7}^{2}+Q \sigma_{\eta}^{2} \Omega_{6}\right\}},
$$

\footnotetext{
${ }^{22}$ The precise conditions under which increases in $\sigma_{\phi}^{2}$ are detrimental to welfare are omitted here due to their complexity and length.

${ }^{23}$ For the complete set of conditions governing each transparency regime see Appendix B.4.
} 
where:

$$
\begin{aligned}
& \Omega_{6} \equiv 1-(1-Q)(2-r) r \\
& \Omega_{7} \equiv 1-(1-Q) r \\
& \Omega_{8} \equiv(1-r)^{2}+Q[1+(2-r) r] .
\end{aligned}
$$

As in the case of intermediate values of $\lambda$, differentiating expected welfare in (2.52) with respect to the transparency tool yields two stationary points, one of which violates the non-negativity requirement for noise variances. The remaining stationary point is non-negative if the following conditions are met:

$$
\left\{\begin{array}{l}
0<Q<Q_{1} \\
0<\sigma_{\phi}^{2}<\sigma_{\phi 1}^{2},
\end{array}\right.
$$

where:

$$
\begin{aligned}
Q_{1} & \equiv \frac{r^{2}-r^{3}}{1+r+r^{2}-r^{3}} \\
\sigma_{\phi 1}^{2} & \equiv \frac{\sigma_{\varepsilon}^{2}}{1-(1-2 Q) r}\left\{\frac{(1-Q)(1-r) r \sqrt{Q[1-(1-Q)(2-r) r]}}{Q[1-(1-Q)(2-r) r]}-(1+r)\right\},
\end{aligned}
$$

where $\sqrt{Q[1-(1-Q)(2-r) r]}$ is defined for admissible values of $r$ and $Q$. For the remaining parameter combinations constrained optimisation yields zero as the optimal value for $\sigma_{\eta}^{2}$.

The welfare outcomes corresponding to the two disclosure regimes are as follows:

$$
\begin{aligned}
& E\left[W_{R}^{\text {Full }}\right]_{\lambda=0}=-\frac{\sigma_{\phi}^{2}\left(\sigma_{\varepsilon}^{2}+Q \sigma_{\phi}^{2}\right)\left\{\sigma_{\varepsilon}^{2}+\left[1-2(1-Q) r+(1-Q) r^{2}\right] \sigma_{\phi}^{2}\right\}}{\left\{\sigma_{\varepsilon}^{2}+[1-(1-Q) r] \sigma_{\phi}^{2}\right\}^{2}} \\
& E\left[W_{R}^{\text {Part }}\right]_{\lambda=0}=-\frac{(1-r)^{2} \sigma_{\varepsilon}^{2} \sigma_{\phi}^{2}}{(1-r)^{2} \sigma_{\varepsilon}^{2}+[1-r(2-2 Q-r+2 \sqrt{Q[1-(1-Q)(2-r) r]})] \sigma_{\phi}^{2}} .
\end{aligned}
$$

James and Lawler (2011) show that in the absence of passive agents $(Q=0)$ the optimal disclosure policy is full opacity $\left(\sigma_{\eta}^{2} \rightarrow \infty\right)$. Thus, cet. par. the introduction of even a small proportion of passive agents requires a reversal of the disclosure policy to either full or intermediate transparency.

Examination of first derivatives of the welfare outcomes in (2.54) and (2.55) 
with respect to $\sigma_{\varepsilon}^{2}$ and $\sigma_{\phi}^{2}$ shows that welfare strictly increases as active agents and the policymaker become better informed. The sign on the derivative with respect to $Q$ indicates that expected welfare is strictly decreasing in the proportion of passive agents. Comparison with the expected welfare outcome in (2.43) evaluated ${ }^{24}$ at $\lambda=0$ reveals that the presence of passive agents has a detrimental effect on welfare, which cannot be fully remedied by choosing the optimal setting of the policy instrument and optimal disclosure policy.

\subsubsection{J-L payoff function $(\lambda=1)$}

Setting $\lambda=1$ in the expected equilibrium welfare expression in (2.49) yields expected welfare expression for the J-L case:

$$
E\left[W_{R}\right]_{\lambda=1}=-\frac{(1-r)^{2}\left(\sigma_{\varepsilon}^{2}+\sigma_{\phi}^{2}+\sigma_{\eta}^{2}\right)\left[\sigma_{\varepsilon}^{2}+Q\left(\sigma_{\phi}^{2}+\sigma_{\eta}^{2}\right)\right] \sigma_{\phi}^{2}}{\sigma_{\varepsilon}^{4}+\sigma_{\varepsilon}^{2}\left[2 \sigma_{\phi}^{2} \Omega_{7}+(1+Q) \sigma_{\eta}^{2}\right]+\left(\sigma_{\phi}^{2}+\sigma_{\eta}^{2}\right)\left[\sigma_{\phi}^{2} \Omega_{7}^{2}+Q \sigma_{\eta}^{2}\right]}
$$

where:

$$
\Omega_{7} \equiv 1-(1-Q) r
$$

Differentiation of (2.56) with respect to $\sigma_{\eta}^{2}$ yields two stationary points, only one of which takes valid (i.e. non-negative) values for some parameter combinations. When the said stationary point is non-positive, either full or zero transparency is optimal. Table 2.1 summarises the conditions for the different optimal disclosure policies. Appendix B.1 presents a numerical example of Table 2.1.

Table 2.1: Conditions for optimal disclosure policies under commitment in the J-L case

\begin{tabular}{|r|c|c|c|}
\hline Optimal Disclosure & Full: $\sigma_{\eta}^{2}=0$ & Partial: $\sigma_{\eta}^{2}=\left(\sigma_{\eta}^{2}\right)^{*}$ & Zero: $\sigma_{\eta}^{2} \rightarrow \infty$ \\
Condition & $0<r \leq r_{1}$ & $r_{1}<r<r_{2}$ & $r_{2} \leq r<1$ \\
\hline
\end{tabular}

The critical value $r_{1}$ is a function of parameters $Q, \sigma_{\varepsilon}^{2}$ and $\sigma_{\phi}^{2}$, whereas critical value $r_{2}$ is a function only of $Q$ :

$$
\begin{aligned}
& r_{1}=\frac{\left(\sigma_{\varepsilon}^{2}+\sigma_{\phi}^{2}\right) \sqrt{Q}}{(1+\sqrt{Q})\left(\sigma_{\varepsilon}^{2}+\sigma_{\phi}^{2} \sqrt{Q}\right)} \\
& r_{2}=\frac{1}{1+\sqrt{Q}} .
\end{aligned}
$$

\footnotetext{
${ }^{24}$ This is equivalent to the welfare outcome in James and Lawler (2011).
} 
The welfare outcomes corresponding to the three disclosure regimes are as follows:

$$
\begin{aligned}
& E\left[W_{R}^{\text {Full }}\right]=-\frac{(1-r)^{2} \sigma_{\phi}^{2}\left(\sigma_{\varepsilon}^{2}+\sigma_{\phi}^{2}\right)\left(\sigma_{\varepsilon}^{2}+Q \sigma_{\phi}^{2}\right)}{\left[\sigma_{\varepsilon}^{2}+(1-r+Q r) \sigma_{\phi}^{2}\right]^{2}} \\
& E\left[W_{R}^{\text {Part }} \mid \theta\right]=-\frac{(1-r)^{2} \sigma_{\varepsilon}^{2} \sigma_{\phi}^{2}}{\sigma_{\varepsilon}^{2}+(1-r-r \sqrt{Q})^{2} \sigma_{\phi}^{2}} \\
& E\left[W_{R}^{\text {Zero }} \mid \theta\right]=-(1-r)^{2} \sigma_{\phi}^{2} .
\end{aligned}
$$

Although, in the absence of passive agents $(Q=0)$, the optimal disclosure policy is full opacity $\left(\sigma_{\eta}^{2} \rightarrow \infty\right)$, the introduction of even a small proportion of passive agents can lead to either full, intermediate, or zero transparency being optimal.

Examination of first derivatives of the welfare outcomes in (2.59)-(2.60) with respect to $\sigma_{\varepsilon}^{2}$ and $\sigma_{\phi}^{2}$ shows that welfare strictly increases as active agents and the policymaker become better informed ${ }^{25}$. The expected welfare under zero disclosure is strictly increasing in the quality of the CB's private information. Expected welfare under full and partial transparency is decreasing in the proportion of passive agents, whereas the welfare outcome under zero transparency is independent of $Q$. Comparison of welfare outcomes with and without passive agents - expression (2.43) evaluated $^{26}$ at $\lambda=1$ and expressions (2.59)-(2.61) - reveals that the presence of passive agents has a detrimental effect on welfare, which cannot be fully remedied by choosing the optimal disclosure policy and instrument adjustment.

In the present section we analysed the interaction of optimal communication policy and the optimal shape of a policy rule. In the following Section we discuss the welfare effects of transparency under discretionary monetary policy.

\subsection{Discretion}

In this Section we solve the model under the assumption that the policymaker conducts monetary policy in discretionary fashion. Following the analytical steps of the previous Section, we begin by looking at a simplified version of the setup without the information stickiness.

\footnotetext{
${ }^{25}$ In the case of expected welfare under full transparency, this is true for parameter values that imply optimality of full transparency, however, outside the relevant set of parameter combinations welfare is increasing as the noisiness of the agents' private signals increases for some parameter values.

${ }^{26}$ This equivalent to the welfare outcome obtained in James and Lawler (2012b).
} 


\subsubsection{Equilibrium without passive agents $(Q=0)$}

In the absence of passive agents $(Q=0)$ the welfare expression is given by (2.38):

$$
\left.W\right|_{Q=0}=\int_{0}^{1}-\left[a_{i}^{A}-(1-\lambda r)(\theta+g)-\lambda r \bar{a}\right]^{2} d i
$$

Knowing that the CB aims to maximise social welfare, market participants use the welfare function to predict what the monetary instrument adjustment is going to be. Differentiating the welfare expression in (2.38) with respect to the policy instrument $g$, solving the first-order condition for $g$ and taking expectations of the resulting expression yields:

$$
g=E_{g}[\bar{a}]-E_{g}[\theta],
$$

where $E_{g}[\cdot]$ is the expectation of a variable conditional on the information set of the CB. Taking expectations of (2.27) and setting $Q=0$ yields:

$$
E_{g}[\bar{a}]=E_{g}\left[\kappa_{0}+\kappa_{1} \theta+\kappa_{2} y\right]=\kappa_{0}+\kappa_{1} E_{g}[\theta]+\kappa_{2} y,
$$

where:

$$
E_{g}[\theta]=z,
$$

since the private signal $z$ is the only information that the CB has about the possible realisation of $\theta$. Thus, optimal policy is given by two expressions

$$
\left\{\begin{array}{l}
g=\kappa_{0}+\left(\kappa_{1}-1\right) z+\kappa_{2} y \\
g=\rho_{0}+\rho_{1} z+\rho_{2} y .
\end{array}\right.
$$

The former is obtained by substituting (2.63) and (2.64) into (2.62) and the latter is taken directly from equation (2.31). Applying the method of undetermined coefficients we obtain the following expressions for optimal policy parameters:

$$
\begin{aligned}
& \rho_{0}^{*}=\kappa_{0} \\
& \rho_{1}^{*}=\kappa_{1}-1 \\
& \rho_{2}^{*}=\kappa_{2} .
\end{aligned}
$$

Substituting the policy parameters (2.66a)-(2.66c) into the policy rule (2.31) and taking expectations of the resulting expression yields agent $i$ 's expectation of the policy adjustment:

$$
E_{i}[g]=\kappa_{0}+\left(\kappa_{1}-1\right) E_{i}[z]+\kappa_{2} y
$$


where $i \in(Q, 1]$ and the expectation of $z$ is given by (2.33). From equations (2.24) and (2.25), the two representations of optimal action:

$$
\left\{\begin{array}{l}
a_{i}^{A}=\left[1-r+(1-\lambda) \lambda r^{2}\right]\left(E_{i}[\theta]+E_{i}[g]\right)+r[1-(1-\lambda) \lambda r] E_{i}[\bar{a}] \\
a_{i}^{A}=\kappa_{0}+\kappa_{1} x_{i}+\kappa_{2} y
\end{array}\right.
$$

Using (2.32), (2.33), (2.67) and (2.35) and applying the method of undetermined coefficients to the two representations of optimal action we get the following equilibrium coefficients:

$$
\begin{aligned}
& \kappa_{0}^{*}=\kappa_{0}^{*} \\
& \kappa_{1}^{*}=\frac{\left[1-r+(1-\lambda) \lambda r^{2}\right] \sigma_{\phi}^{2}}{\sigma_{\varepsilon}^{2}+\left[1-r+(1-\lambda) \lambda r^{2}\right] \sigma_{\phi}^{2}} \\
& \kappa_{2}^{*}=\kappa_{2}^{*} .
\end{aligned}
$$

The equilibrium coefficients (2.69a)-(2.69c) imply that there is a continuum of equilibria, since $\kappa_{0}^{*}$ and $\kappa_{2}^{*}$ have indeterminate values ${ }^{27}$. Given a set of parameter values, the expression in (2.69b) is fixed, which means that the weight individual agents place on their private information is the same across the population and across equilibria. The same is true about the policymaker's response to the private signal $z$, which is given by (2.66b) and is the same for every equilibrium. In contrast to that, each equilibrium is defined by a unique combination of intercepts and responses to the public signal $\left(\kappa_{0}^{*}=\rho_{0}^{*}, \kappa_{2}^{*}=\rho_{2}^{*}\right)$.

In order to find the level of social welfare that corresponds to every equilibrium, we substitute optimal policy parameters (2.66a)-(2.66c) and the equilibrium coefficient (2.69b) into (2.39), which yields the ex ante expected equilibrium welfare:

$$
E\left[W_{D}\right]_{Q=0}=-\frac{(1-\lambda r)^{2} \sigma_{\varepsilon}^{2} \sigma_{\phi}^{2}\left\{\sigma_{\varepsilon}^{2}+[1-(1-\lambda) r]^{2} \sigma_{\phi}^{2}\right\}}{\left\{\sigma_{\varepsilon}^{2}+\left[1-r+(1-\lambda) \lambda r^{2}\right] \sigma_{\phi}^{2}\right\}^{2}}
$$

The outcome in (2.70) is the same for all equilibria, as it does not depend on $\kappa_{0}$ nor $\kappa_{2}$. It should also be noted, that the welfare outcome in (2.70) is independent of $\sigma_{\eta}^{2}$, which implies that the choice of a transparency regime in this case is irrelevant from a social perspective.

In the following Section we re-introduce the assumption of information stickiness and derive the equilibrium for non-zero values of $Q$. Once the solution is obtained,

\footnotetext{
${ }^{27}$ James and Lawler (2011) find that under discretion their model, similarly, produces multiplicity of equilibria.
} 
we perform a number of exercises, including setting $Q=0$ to see if this allows us to overcome the problem of the multiplicity of equilibria under discretion that we observe in the current Section.

\subsubsection{Equilibrium with passive agents $(0<Q<1)$}

Following the same steps as in the previous Section, we begin by determining the optimal policy intervention, given intermediate levels of information stickiness $(0<Q<1)$ and the welfare expression (2.28). Differentiating the welfare function with respect to $g$ and solving the resulting first-order condition yields an expression identical to that in (2.62):

$$
g=E_{g}[\bar{a}]-E_{g}[\theta] .
$$

Taking expectations of the average action in (2.27) and using $E_{g}[\theta]=z$ from (2.64), we obtain the CB's expectation of the average action:

$$
E_{g}[\bar{a}]=E_{g}\left[(1-Q)\left(\kappa_{0}+\kappa_{1} \theta+\kappa_{2} y\right)\right]=(1-Q)\left(\kappa_{0}+\kappa_{1} z+\kappa_{2} y\right) .
$$

Substituting (2.64) and (2.71) into (2.62) yields an expression for optimal policy that can be compared to the optimal policy in general form from (2.31):

$$
\left\{\begin{array}{l}
g=(1-Q) \kappa_{0}+\left[(1-Q) \kappa_{1}-1\right] z+(1-Q) \kappa_{2} y \\
g=\rho_{0}+\rho_{1} z+\rho_{2} y
\end{array}\right.
$$

By comparing the coefficients on the two representations of $g$ we arrive at the following values of optimal policy parameters:

$$
\begin{aligned}
& \rho_{0}^{*}=(1-Q) \kappa_{0} \\
& \rho_{1}^{*}=(1-Q) \kappa_{1}-1 \\
& \rho_{2}^{*}=(1-Q) \kappa_{2} .
\end{aligned}
$$

Given the policy parameters (2.73a)-(2.73c), agent $i$ 's expectation of the policy instrument adjustment is:

$$
E_{i}[g]=(1-Q) \kappa_{0}+\left[(1-Q) \kappa_{1}-1\right] E_{i}[z]+(1-Q) \kappa_{2} y
$$

Under sticky information, agent $i$ 's expectation of average action becomes:

$$
E_{i}[\bar{a}]=(1-Q) \kappa_{0}+(1-Q) \kappa_{1} E_{i}[\theta]+(1-Q) \kappa_{2} y .
$$

Substituting (2.32), (2.33), (2.74) and (2.75) into optimal action (2.24) and comparing the resulting coefficients with those in (2.25) yields the following equilibrium 
coefficients:

$$
\begin{aligned}
\kappa_{0}^{*} & =0 \\
\kappa_{1}^{*} & =\frac{\left[1-r+(1-\lambda) \lambda r^{2}\right] \sigma_{\phi}^{2}}{\sigma_{\varepsilon}^{2}+\{1-(1-Q)[1-r(1-\lambda) \lambda] r\} \sigma_{\phi}^{2}+Q \sigma_{\eta}^{2}} \\
\kappa_{2}^{*} & =-\kappa_{1}
\end{aligned}
$$

where $\kappa_{0}^{*}+\kappa_{1}^{*}+\kappa_{2}^{*}=0$. Thus, active agent's equilibrium action is:

$$
a_{i}^{A}=\kappa_{1}^{*}\left(x_{i}-y\right)
$$

and the average action is:

$$
\bar{a}=(1-Q) \kappa_{1}^{*}(\theta-y) .
$$

Given the optimal parameter values (2.73a)-(2.73c), equilibrium weights (2.76a)$(2.76 \mathrm{c})$ and the definition for the public signal $y$, the optimal policy expression (2.31) can be reformulated in the following way:

$$
g=-z+\rho_{2}^{*} \eta=-z-\frac{(1-Q)\left[1-r+(1-\lambda) \lambda r^{2}\right] \sigma_{\phi}^{2}}{\sigma_{\varepsilon}^{2}+\{1-(1-Q)[1-(1-\lambda) \lambda r] r\} \sigma_{\phi}^{2}+Q \sigma_{\eta}^{2}} \eta
$$

This formulation of the policy rule uses the fact that $\rho_{0}^{*}+\rho_{1}^{*}+\rho_{2}^{*}=-1$. It should be noted that the $\mathrm{CB}$ offsets its estimate of $\theta$ one-to-one, but also takes into account the effect of the additional noise in the public signal on equilibrium actions in the market.

Substituting the optimal policy parameters in (2.73a)-(2.73c) as well as equilibrium weights (2.76a)-(2.76c) into the expression for expected welfare (see Appendix B.2) yields the expected equilibrium welfare outcome under discretionary optimal policy:

$$
\begin{aligned}
E\left[W_{D}\right]= & -(1-\lambda r)^{2} \sigma_{\phi}^{2}\left[\sigma_{\varepsilon}^{2}+Q\left(\sigma_{\phi}^{2}+\sigma_{\eta}^{2}\right)\right] . \\
& \cdot \frac{\left(\sigma_{\varepsilon}^{2}+\{1-(1-\lambda)(1-Q)[2-(1-\lambda) r] r\} \sigma_{\phi}^{2}+Q \sigma_{\eta}^{2}\right)}{\left(\sigma_{\varepsilon}^{2}+\{1-(1-Q)[1-r(1-\lambda) \lambda] r\} \sigma_{\phi}^{2}+Q \sigma_{\eta}^{2}\right)^{2}} .
\end{aligned}
$$

The CB chooses the appropriate level of transparency, given the expected equilibrium welfare expression in (2.80). As before, we proceed by looking at optimal disclosure policy for three separate cases in respect of information stickiness. We begin with the general case of $0<\lambda<1$, which is followed by separate discussions of transparency in the extreme case of $\lambda=0$ and $\lambda=1$. 


\subsubsection{Hybrid payoff function $(0<\lambda<1)$}

The expected equilibrium welfare function in (2.80) is continuous in the variance of the additional noise $\sigma_{\eta}^{2}$, allowing us to use differentiation. The sign of the first derivative of expected equilibrium welfare (2.80) with respect to the transparency tool $\sigma_{\eta}^{2}$ is ambiguous. Setting the first derivative equal to zero and solving the resulting first-order condition yields:

$$
\sigma_{\eta 1}^{2}=\frac{(3 \lambda r-1-r) \sigma_{\varepsilon}^{2}-\Omega_{9} \sigma_{\phi}^{2}}{Q[1-(3 \lambda-1) r]}
$$

where $\Omega_{9} \equiv 1-(2+4 Q \lambda-3 Q-\lambda) r+\left(1-\lambda^{2}\right)(1-Q) r^{2}-(1-\lambda)^{2} \lambda(1-Q) r^{3}$. The solution in (2.81) is non-negative for a subset of parameter values ${ }^{28}$, thus we consider $\sigma_{\eta 1}^{2}$ to be not defined for the remaining combinations of parameter values. Performing the second-derivative test reveals that the expected equilibrium welfare has a local minimum at the stationary point $\sigma_{\eta 1}^{2}$, when $0 \leq \sigma_{\eta 1}^{2}$. When the stationary point $\sigma_{\eta 1}^{2}$ is not defined, the expected welfare outcome is either monotonically increasing or monotonically decreasing in $\sigma_{\eta}^{2}$ over the range $0 \leq \sigma_{\eta}^{2}$, depending on parameter values $^{29}$.

Since the expected welfare function is continuous in $\sigma_{\eta}^{2}$ and the only stationary point with respect to $\sigma_{\eta}^{2}$ minimises welfare, partial transparency is a suboptimal disclosure policy. To assess which of the remaining two transparency regimes - full or zero - is optimal we compare the respective expected equilibrium welfare outcomes. The welfare outcome under full transparency is obtained by setting $\sigma_{\eta}^{2}=0$ in (2.80):

$E\left[W_{D}^{F u l l}\right]=-\frac{(1-\lambda r)^{2} \sigma_{\phi}^{2}\left(\sigma_{\varepsilon}^{2}+Q \sigma_{\phi}^{2}\right)\left(\sigma_{\varepsilon}^{2}+\{1-(1-\lambda)(1-Q)(2-(1-\lambda) r) r\} \sigma_{\phi}^{2}\right)}{\left\{\sigma_{\varepsilon}^{2}+\left[1-(1-Q) r+(1-\lambda)(1-Q) \lambda r^{2}\right] \sigma_{\phi}^{2}\right\}^{2}}$

whereas the welfare outcome under zero transparency corresponds to setting $\sigma_{\eta}^{2} \rightarrow$ $\infty$ :

$$
E\left[W_{D}^{Z e r o}\right]=-(1-\lambda r)^{2} \sigma_{\phi}^{2}
$$

It should be noted that, expected welfare outcomes under full and zero transparency are identical to those under commitment, i.e. expressions (2.50) and (2.51), respectively. We return to this point at a later stage, when we compare the performance of the two monetary policy regimes. Comparison of expressions (2.82) and (2.83) reveals that expected equilibrium welfare under full transparency can be re-expressed

\footnotetext{
${ }^{28}$ See Appendix B.6 for conditions under which $\sigma_{\eta 1}^{2}$ is defined.

${ }^{29}$ See Appendix B.6 for a broad classification of shapes of the welfare function, corresponding conditions on parameter values and graphical representation of numerical examples.
} 
as follows:

$$
E\left[W_{D}^{\text {Full }}\right]=\Omega_{10} E\left[W_{D}^{\text {Zero }}\right],
$$

where:

$$
\Omega_{10} \equiv \frac{\left(\sigma_{\varepsilon}^{2}+Q \sigma_{\phi}^{2}\right)\left\{\sigma_{\varepsilon}^{2}+[1-(1-\lambda)(1-Q)(2-(1-\lambda) r) r] \sigma_{\phi}^{2}\right\}}{\left\{\sigma_{\varepsilon}^{2}+\left[1-(1-Q) r+(1-\lambda)(1-Q) \lambda r^{2}\right] \sigma_{\phi}^{2}\right\}^{2}}>0 .
$$

Thus, whether or not $\Omega_{10}$ is greater than unity determines which disclosure regime is preferable.

The comparison of welfare outcomes under full and zero disclosure reveals that zero disclosure is preferable if one of the following two sets of conditions is satisfied:

$$
\left\{\begin{array}{l}
\frac{2}{3}<\lambda<1 \\
\frac{1}{3 \lambda-1}<r<1 \\
0<Q<Q_{2} \\
0<\sigma_{\phi}^{2} \leq \sigma_{\phi 2}^{2},
\end{array}\right.
$$

or:

$$
\left\{\begin{array}{l}
\frac{2}{3}<\lambda<1 \\
\frac{1}{3 \lambda-1}<r<1 \\
Q_{2} \leq Q<1,
\end{array}\right.
$$

where:

$$
\begin{aligned}
Q_{2} & \equiv \frac{[1-(1-\lambda) r](1-\lambda r)^{2}}{\{2+[2-(1+r(1-\lambda)) \lambda] r\} \lambda r-2 r} \\
\sigma_{\phi 2}^{2} & \equiv \frac{(3 \lambda r-1-r) \sigma_{\varepsilon}^{2}}{1-[1-2 Q(1-\lambda)+\lambda] r+(2-\lambda) \lambda(1-Q) r^{2}-(1-\lambda) \lambda^{2}(1-Q) r^{3}} .
\end{aligned}
$$

The two welfare outcomes are equivalent when:

$$
\left\{\begin{array}{l}
\frac{2}{3}<\lambda<1 \\
\frac{1}{3 \lambda-1}<r<1 \\
0<Q<Q_{2} \\
0<\sigma_{\phi}^{2}=\sigma_{\phi 2}^{2},
\end{array}\right.
$$


and full transparency is superior for the rest of parameter combinations:

$$
0<\lambda \leq \frac{2}{3}
$$

or:

$$
\left\{\begin{array} { l l } 
{ \frac { 2 } { 3 } < \lambda < 1 } & { ( 2 . 8 9 \mathrm { a } ) } \\
{ 0 < r \leq \frac { 1 } { 3 \lambda - 1 } , } & { ( 2 . 8 9 \mathrm { b } ) }
\end{array} \quad \left\{\begin{array}{l}
\frac{2}{3}<\lambda<1 \\
\frac{1}{3 \lambda-1}<r<1 \\
0<Q<Q_{2} \\
0<\sigma_{\phi 2}^{2} \leq \sigma_{\phi}^{2} .
\end{array}\right.\right.
$$

It should be noted, that $\lambda \leq \frac{2}{3}$ is a sufficient condition for full transparency to be the optimal disclosure policy.

\subsubsection{M-S payoff function $(\lambda=0)$}

We begin by setting $\lambda=0$ in the expected equilibrium welfare expression (2.80):

$$
E\left[W_{D}\right]_{\lambda=0}=-\frac{\sigma_{\phi}^{2}\left[\sigma_{\varepsilon}^{2}+Q\left(\sigma_{\phi}^{2}+\sigma_{\eta}^{2}\right)\right]\left\{\sigma_{\varepsilon}^{2}+[1-(1-Q)(2-r) r] \sigma_{\phi}^{2}+Q \sigma_{\eta}^{2}\right\}}{\left\{\sigma_{\varepsilon}^{2}+[1-(1-Q) r] \sigma_{\phi}^{2}+Q \sigma_{\eta}^{2}\right\}^{2}} .
$$

Expected equilibrium welfare (2.91) is decreasing in the variance of the additional noise $\sigma_{\eta}^{2}$ for all admissible parameter values and $0<Q<1$. Thus, full transparency is optimal and the corresponding expected welfare outcome is given by:

$$
E\left[W_{D}^{F u l l}\right]_{\lambda=0}=-\sigma_{\phi}^{2} \frac{\left(\sigma_{\varepsilon}^{2}+Q \sigma_{\phi}^{2}\right)\left\{\sigma_{\varepsilon}^{2}+[1-(1-Q)(2-r) r s] \sigma_{\phi}^{2}\right\}}{\left\{\sigma_{\varepsilon}^{2}+[1-(1-Q) r] \sigma_{\phi}^{2}\right\}^{2}} .
$$

Examination of first derivatives of (2.92) with respect to noise variances shows, that expected welfare decreases as the private sector becomes less informed (as $\sigma_{\varepsilon}^{2}$ increases). The sign of the first derivative with respect to the variance of the noise of the CB's private signal $\sigma_{\phi}^{2}$ is ambiguous and depends on parameter values. Restricting the parameter set to the case where the CB is better informed than the private sector $\left(\sigma_{\phi}^{2}<\sigma_{\varepsilon}^{2}\right)$ is sufficient to ensure that welfare is increasing in the quality of CB's information. Expected welfare is decreasing in the proportion of passive agents $Q$, which is true regardless of how $\sigma_{\phi}^{2}$ compares to $\sigma_{\varepsilon}^{2}$. 


\subsubsection{J-L payoff function $(\lambda=1)$}

Setting $\lambda=1$ in the expected equilibrium welfare equation (2.80) yields:

$$
E\left[W_{D}\right]_{\lambda=1}=-\frac{(1-r)^{2} \sigma_{\phi}^{2}\left(\sigma_{\varepsilon}^{2}+\sigma_{\phi}^{2}+Q \sigma_{\eta}^{2}\right)\left[\sigma_{\varepsilon}^{2}+Q\left(\sigma_{\phi}^{2}+\sigma_{\eta}^{2}\right)\right]}{\left\{\sigma_{\varepsilon}^{2}+[1-(1-Q) r] \sigma_{\phi}^{2}+Q \sigma_{\eta}^{2}\right\}^{2}} .
$$

Differentiating expected equilibrium welfare (2.93) with respect to $\sigma_{\eta}^{2}$ yields one stationary point:

$$
\sigma_{\eta 2}^{2}=\frac{(2 r-1) \sigma_{\varepsilon}^{2}-(1-r-Q r) \sigma_{\phi}^{2}}{Q(1-2 r)} .
$$

Performing the second-derivative test reveals that the expected equilibrium welfare has a local minimum at the stationary point $\sigma_{\eta 2}^{2}$. In line with this result, the welfare outcomes evaluated at full transparency $\left(\sigma_{\eta}^{2}=0\right)$ :

$$
E\left[W_{D}^{\text {Full }}\right]_{\lambda=1}=-\frac{(1-r)^{2} \sigma_{\phi}^{2}\left(\sigma_{\varepsilon}^{2}+\sigma_{\phi}^{2}\right)\left(\sigma_{\varepsilon}^{2}+Q \sigma_{\phi}^{2}\right)}{\left[\sigma_{\varepsilon}^{2}+(1-r+Q r) \sigma_{\phi}^{2}\right]^{2}},
$$

and zero transparency $\left(\sigma_{\eta}^{2} \rightarrow \infty\right)$ :

$$
E\left[W_{D}^{Z e r o}\right]_{\lambda=1}=-(1-r)^{2} \sigma_{\phi}^{2},
$$

strictly dominate the outcome with partial transparency $\left(\sigma_{\eta}^{2}=\sigma_{\eta 2}^{2}\right)$ for all parameter values that result in $\sigma_{\eta 2}^{2}$ being positive. It should be noted, that outcomes (2.59) and (2.61) are identical to those under discretion - (2.95) and (2.96), respectively.

The decision between full and zero transparency depends on parameter values. The conditions for the optimality of the two transparency policies are presented in Table 2.2, where they are expressed in terms of a restriction on $r$.

Table 2.2: Conditions for optimal disclosure policies under discretion in the J-L case

\begin{tabular}{|r|c|c|}
\hline Optimal Disclosure & Full: $\sigma_{\eta}^{2}=0$ & Zero: $\sigma_{\eta}^{2} \rightarrow \infty$ \\
Condition & $0<r \leq r_{3}$ & $r_{3} \leq r<1$ \\
\hline
\end{tabular}

The critical value $r_{3}$ is a function of variances $\sigma_{\varepsilon}^{2}$ and $\sigma_{\phi}^{2}$ and the proportion of passive agents $0<Q<1$ :

$$
r_{3}=\frac{\sigma_{\varepsilon}^{2}+\sigma_{\phi}^{2}-\sqrt{\left(\sigma_{\varepsilon}^{2}+\sigma_{\phi}^{2}\right)\left(\sigma_{\varepsilon}^{2}+Q \sigma_{\phi}^{2}\right)}}{(1-Q) \sigma_{\phi}^{2}}
$$


which implies that the condition for superiority of one transparency regime over the other is defined in terms of combinations of all four parameters, rather than just a restriction on $r$. Here this condition is expressed in terms of $r$ for convenience of presentation. It should be noted, that the two transparency regimes yield the same expected welfare outcome when $r=r_{3}$. A numerical example and a graphical representation of the optimality conditions are presented in Appendix B.7.

\subsubsection{Equilibrium without passive agents re-attempted $(Q \rightarrow$} $0)$

Here we attempt to overcome the indeterminacy issue that arose in the setup without passive agents by using the solution from the previous section, where passive agents are present $(0<Q<1)$, and setting the proportion of passive agents equal to zero. Setting $Q=0$ in (2.76b) and substituting it into (2.77) yields active agent $i$ 's optimal action:

$$
a_{i}^{A}=\frac{\left[1-r+(1-\lambda) \lambda r^{2}\right] \sigma_{\phi}^{2}}{\sigma_{\varepsilon}^{2}+\left[1-r+(1-\lambda) \lambda r^{2}\right] \sigma_{\phi}^{2}}\left(x_{i}-y\right)
$$

In the absence of passive agents, the optimal policy expression (2.79) becomes:

$$
g=-z-\frac{\left[1-r+(1-\lambda) \lambda r^{2}\right] \sigma_{\phi}^{2}}{\sigma_{\varepsilon}^{2}+\left[1-r+(1-\lambda) \lambda r^{2}\right] \sigma_{\phi}^{2}} \eta,
$$

and the expected equilibrium welfare in (2.80) simplifies to:

$$
E\left[W_{D}\right]_{Q=0}=-\frac{(1-\lambda r)^{2} \sigma_{\varepsilon}^{2} \sigma_{\phi}^{2}\left\{\sigma_{\varepsilon}^{2}+[1-(1-\lambda) r]^{2} \sigma_{\phi}^{2}\right\}}{\left\{\sigma_{\varepsilon}^{2}+\left[1-r+(1-\lambda) \lambda r^{2}\right] \sigma_{\phi}^{2}\right\}^{2}}
$$

It is apparent from (2.100) that the decision about the transparency tool $\sigma_{\eta}^{2}$ becomes irrelevant. It should be noted that the welfare outcome in (2.100) is equivalent to that of a setup, where no direct policy intervention is assumed and full transparency is employed. This welfare outcome is also equivalent to that in the first attempt at solving the model without passive agents that lead to multiplicity of equilibria (see equation (2.70)). Thus, this method allows us to isolate a single equilibrium for the case where all agents are active.

Comparison of welfare outcomes with and without passive agents - expressions (2.82)-(2.83) and expression (2.100) - reveals the following. If full transparency is optimal in the presence of passive agents (see conditions (2.88), (2.89a)-(2.89b) and (2.90a)-(2.86c)), then the absence of passive agents guarantees a superior welfare outcome. However, if zero transparency is optimal, expected welfare becomes 
independent of $Q$ and the comparison with the welfare outcome in the absence of information stickiness yields an ambiguous result. To be more precise, the relationship between expression (2.83) and expression (2.100) is as follows:

$$
E\left[W_{D}\right]_{Q=0}<E\left[W_{D}^{Z e r o}\right]
$$

for:

$$
\left\{\begin{array}{l}
\frac{2}{3}<\lambda \leq 1 \\
\frac{1}{3 \lambda-1}<r<1 \\
\frac{[1-(1-\lambda) r](1-\lambda r)^{2}}{(3 \lambda-1) r-1}<\frac{\sigma_{\varepsilon}^{2}}{\sigma_{\phi}^{2}} \\
Q_{2} \leq Q<1
\end{array}\right.
$$

where:

$$
Q_{2} \equiv \frac{[1-(1-\lambda) r](1-\lambda r)^{2}}{\{2+[2-(1+r(1-\lambda)) \lambda] r\} \lambda r-2 r} .
$$

This implies that for some combinations of $\lambda, r, \sigma_{\varepsilon}^{2}$ and $\sigma_{\phi}^{2}$ optimal policy and zero transparency in the presence of passive agents performs better than optimal policy in the absence of passive agents. In other words, given two economies - A and B characterised by the same values of $\sigma_{\varepsilon}^{2}, \sigma_{\phi}^{2}, \lambda$ and $r$, that satisfy conditions (2.102a)(2.102c), if passive agents are absent from economy $\mathrm{A}(Q=0)$ and present ${ }^{30}$ in economy B (subject to $Q_{2} \leq Q<1$ ), optimal policy intervention and any degree of disclosure in economy A perform worse, in terms of social welfare, than zero transparency and optimal policy intervention in economy B. The combination of optimal policy and complete opacity about the policymaker's private information offsets the negative impact of the presence of passive agents in economy B.

\subsubsection{Equilibrium without active agents $(Q=1)$}

In this Section we briefly look at the special case of an economy populated entirely by passive agents who ignore all new information. Since the term new information also includes public announcements about the shock, central bank transparency considerations become irrelevant in the current context. As suggested by expression (2.20), in an economy characterised by $Q=1$ every agent chooses the optimal action based on the unconditional expected value of the state variable $E[\theta]=0$. In other words, in the absence of any additional information all agents choose not to act at

\footnotetext{
${ }^{30} \mathrm{As} \lambda \rightarrow 1$ and $r \rightarrow \frac{1}{2}$, condition (2.102d) restricts $Q$ to values close to unity. Alternatively, in the limit, as $\lambda \rightarrow 1$ and $r \rightarrow 1$, any value of $Q$ between zero and unity is permissible. Thus, the welfare outcome in economy $\mathrm{B}$ is more likely to be superior when the coordination motive is stronger and the payoff function is closer to the J-L case.
} 
all:

$$
a_{i}^{P}=E_{i}\left[a_{j}^{P}\right]=\bar{a}=E_{i}[\bar{a}]=E_{g}[\bar{a}]=0,
$$

where $i \in[0,1], j \in[0,1]$ and $i \neq j$. Equality in (2.103) further shows that there is no action dispersion across the population, hence the average action is also equal to zero. Moreover, every agent expects every other agent not to act and the average action to be zero. The latter expectation is also formed by the policymaker.

Setting $Q=1$ in the expression for social welfare (2.28) and using $\bar{a}=0$ yields:

$$
W=-(1-\lambda r)^{2}(\theta+g)^{2}
$$

Social welfare in (2.104) depends on the policymaker's ability to offset the shock $\theta$ through the appropriate policy instrument adjustment $g$. Differentiating the welfare expression in (2.104) with respect to $g$, solving the first-order condition and taking expectations of the resulting expression gives:

$$
g=-E_{g}[\theta]
$$

where $E_{g}[\theta]=z$, from (2.64). Thus optimal policy is given by:

$$
g=-z
$$

which corresponds to the following optimal policy parameter values:

$$
\begin{aligned}
& \rho_{0}^{*}=0 \\
& \rho_{1}^{*}=-1 .
\end{aligned}
$$

It should be noted, that the assumption about the monetary policy regime plays no role in determining the optimal instrument adjustment. The shape of the policy function given in (2.106) applies to both the case of commitment and the case of discretion. Under the former regime and given that the private sector does not have access to any information about the shock, it is optimal for the policymaker to commit to fully offsetting the shock to the best of its knowledge. Under the latter regime and given private sector's expectations about policy, a benevolent policymaker has no incentive to deviate from those expectations. Thus, the use of subscripts $D$ and $R$ in relation to the $Q=1$ case is redundant.

To obtain the expected equilibrium outcome we substitute the optimal policy adjustment from (2.106) and the definition of the policymaker's private signal $z$ from (2.29) into the welfare equation (2.104) and take expectations of the resulting 
expression, which yields:

$$
E[W]_{Q=1}=-(1-\lambda r)^{2} \sigma_{\phi}^{2}
$$

In the case of the M-S payoff function $(\lambda=0)$, expression (2.108) simplifies to $-\sigma_{\phi}^{2}$. Thus, the expected welfare outcome depends solely on the quality of policymaker's private information, the latter directly affecting the policymaker's ability to offset the shock. In the case of the J-L payoff function $(\lambda=1)$ as well as the hybrid payoff functions $(0<\lambda<1)$, expected welfare also depends on the strength of the coordination motive represented by $r$. The stronger the coordination motive the less dependent private sector agents' payoffs are on the market fundamentals, which is borne out by the sign of the derivative of (2.108) with respect to $r$ being positive for $0<\lambda \leq 1$.

The expected welfare outcome under the assumption of all agents being passive, represented by equation (2.108), is equivalent to equilibrium welfare outcomes in the settings characterised by intermediate levels ${ }^{31}$ of information stickiness $(0<Q<1)$, zero disclosure $\left(\sigma_{\eta}^{2} \rightarrow \infty\right)$ and either commitment or discretion, given by equations (2.51) and (2.83), respectively. Active agents observe a completely uninformative public announcement and ignore it, thus active agent $i$ 's expectations of $\theta$ and $z$ become:

$$
E_{i}[\theta]=E_{i}[z]=x_{i}
$$

where $i \in(Q, 1]$. Under commitment, an active agent $i$ forms the following expectations about policy adjustment:

$$
E_{i}[g]=\rho_{0}+\rho_{1} x_{i}
$$

given expression (2.34) as well as expectations in (2.109). Similarly, given (2.35) and (2.109), an active agent $i$ expects the average action across the population to be:

$$
E_{i}[\bar{a}]=(1-Q)\left(\kappa_{0}+\kappa_{1} x_{i}\right)
$$

When (2.110) and (2.111) are substituted into the first of the two representations of optimal action:

$$
\left\{\begin{array}{l}
a_{i}^{A}=\left[1-r+(1-\lambda) \lambda r^{2}\right]\left(E_{i}[\theta]+E_{i}[g]\right)+r[1-(1-\lambda) \lambda r] E_{i}[\bar{a}] \\
a_{i}^{A}=\kappa_{0}+\kappa_{1} x_{i}
\end{array}\right.
$$

\footnotetext{
${ }^{31}$ In the absence of passive agents $(Q=0)$ welfare outcomes under both policy regimes differ from that in equation (2.108).
} 
from (2.24) and (2.25), the method of undetermined coefficients yields:

$$
\begin{aligned}
& \kappa_{0}^{*}=\rho_{0} \\
& \kappa_{1}^{*}=1+\rho_{1} .
\end{aligned}
$$

We then substitute (2.113a)-(2.113b) along with $\kappa_{2}=0$ and $\rho_{2}=0$ into the expected welfare equation (B.20) and maximise the resulting expression with respect to the policy parameters, arriving $a t^{32}$ :

$$
\begin{aligned}
& \rho_{0}^{*}=0 \\
& \rho_{1}^{*}=-1 .
\end{aligned}
$$

Policy parameters (2.114a)-(2.114b) imply that it is optimal for the policymaker to commit to fully offsetting the shock, which is equivalent to the results obtained in the $Q=1$ case and represented by expressions (2.106) and (2.107a)-(2.107b). The policymaker actively discourages active agents to react to their heterogeneous private signals to reduce the welfare loss associated with dispersion of actions. Combining coefficients in (2.113a)-(2.113b) and (2.114a)-(2.114b) demonstrates that under commitment active agents choose to ignore their private information.

As far as zero transparency under discretion is concerned, private agents expect the policymaker to maximise expected social welfare and adjust the policy instrument as specified in expression (2.62):

$$
g=E_{g}[\bar{a}]-E_{g}[\theta],
$$

where:

$$
E_{g}[\bar{a}]=E_{g}\left[(1-Q)\left(\kappa_{0}+\kappa_{1} \theta\right)\right]=(1-Q)\left(\kappa_{0}+\kappa_{1} z\right)
$$

and $E_{g}[\theta]=z$ from (2.64). Assuming that the general form of the instrument adjustment is given by $g=\rho_{0}+\rho_{1} z$, the method of undetermined coefficients yields:

$$
\begin{aligned}
& \rho_{0}^{*}=(1-Q) \kappa_{0} \\
& \rho_{1}^{*}=(1-Q) \kappa_{1}-1 .
\end{aligned}
$$

Optimal policy parameters in (2.116a)-(2.116b) imply that an active agent $i$ expects the instrument adjustment to be as follows:

$$
E_{i}[g]=(1-Q) \kappa_{0}+(1-Q) \kappa_{1} E_{i}[z]=(1-Q) \kappa_{0}+(1-Q) \kappa_{1} x_{i},
$$

\footnotetext{
${ }^{32}$ We obtain the optimal value of $\rho_{1}$ by imposing a parameter restriction so as to prevent the welfare expression from approaching infinity.
} 
where we used (2.109). Substituting (2.109) along with (2.111) and (2.117) into the first of the two optimal action expressions:

$$
\left\{\begin{array}{l}
a_{i}^{A}=\left[1-r+(1-\lambda) \lambda r^{2}\right]\left(E_{i}[\theta]+E_{i}[g]\right)+r[1-(1-\lambda) \lambda r] E_{i}[\bar{a}] \\
a_{i}^{A}=\kappa_{0}+\kappa_{1} x_{i}
\end{array}\right.
$$

and applying the method of undetermined coefficients gives:

$$
\begin{aligned}
& \kappa_{0}^{*}=0 \\
& \kappa_{1}^{*}=0,
\end{aligned}
$$

which, in turn, implies that it is optimal for the policymaker to fully offset the shock by choosing $\rho_{0}^{*}=0$ and $\rho_{1}^{*}=-1$. Given the policymaker's objective function, active agents form a rational expectation about discretionary policy intervention, which discourages them to act on their private information and adjust their action away from zero. Substituting $\kappa_{0}^{*}=\kappa_{1}^{*}=0$ into (2.111) yields $E_{i}[\bar{a}]=0$, showing that an active agent $i$ expects the average action across the population to be zero. Thus, the desire to coordinate one's actions with the actions of other agents also implies a zero-value optimal action strategy for agent $i$. This leads to active agents following a passive strategy and yields a welfare outcome equivalent to that under commitment or complete information stickiness.

In case of both policy regimes, although a non-zero fraction $1-Q$ of agents are active, a representative active agent still chooses not to act thereby emulating the setting where all agents are passive. As active agents choose to ignore their heterogeneous private signals, action dispersion is eliminated, which leads to an expected welfare outcome that is independent of the quality of the private sector's information. Furthermore, the fact that all active agents follow a passive strategy implies that the welfare outcome is independent of $Q$.

In the following Section we proceed by comparing the expected welfare outcomes under the two monetary policy regimes - commitment and discretion. We also look at the implications that each policy regime has for central bank transparency.

\subsection{Optimal Monetary Policy Regime}

In the above Sections we solved the sticky information model under two monetary policy regimes - commitment and discretion. In the latter case market participants were able to infer the shape of the CB's reaction function based on the bank's objectives, whereas in the former case the precise shape of the policy rule was known 
to the market from the start. Now we are in the position to evaluate the relative performance of the two policy regimes in terms of expected social welfare.

In both Sections we first looked at a simplified version of the model with no information stickiness, which corresponds to $Q=0$. For the special cases of the payoff function, referred to as the M-S type $(\lambda=0)$ and J-L type $(\lambda=1)$, we merely reproduced the result reported by James and Lawler (2011), who point out that commitment performs strictly better than discretion in terms of expected welfare. Our findings reinforce James and Lawler's argument by extending this result to hybrid payoff functions corresponding to $\lambda \in(0,1)$. Before transparency considerations are taken into account, comparison of welfare outcomes under commitment and discretion, from equations (2.42) and (2.70), respectively, shows that commitment performs at least as well as discretion:

$$
E\left[W_{D}\right]_{Q=0} \leq E\left[W_{R}\right]_{Q=0}
$$

where the two expressions are identical for $\lambda=0$ and $\sigma_{\eta}^{2}=0$, which corresponds to the M-S payoff function and full transparency. However, taking optimal choice of the degree of disclosure into account renders the inequality in (2.120) strict:

$$
E\left[W_{D}\right]_{Q=0}<E\left[W_{R}^{Z e r o}\right]_{Q=0}
$$

from (2.43) and (2.70). Thus, in the absence of passive agents commitment to a rule in conjunction with full opacity is expected to yield a superior welfare outcome compared to discretionary monetary policy with an arbitrary degree of transparency. This result constitutes Proposition 2.1.

Proposition 2.1: If all private agents are active and aim to maximise a payoff function characterised by $\lambda \in(0,1)$, it is optimal to commit to a monetary policy rule and exercise zero transparency with respect to the $C B$ 's private signal.

Re-introduction of information stickiness into the model $(0<Q<1)$ has implications for the optimal choice of the policy regime as well as optimal degree of transparency. Table 2.3 presents optimal transparency policies depending on the monetary policy regime and the type of the private agents' payoff function. Where more than one transparency regime is identified, the choice depends on parameter values. One thing that is immediately apparent from Table 2.3 is that commitment creates the possibility for partial disclosure to be optimal, whereas under discretion only the extreme cases of transparency can be optimal. As we demonstrate below, the case of partial transparency plays a crucial role in the decision between the two 
Table 2.3: Optimal disclosure policies under commitment and discretion

\begin{tabular}{|r|c|c|c|}
\hline Payoff Function & M-S type: $\lambda=0$ & Hybrid: $0<\lambda<1$ & J-L type: $\lambda=1$ \\
Commitment & Full, Partial & Full, Partial, Zero & Full, Partial, Zero \\
Discretion & Full & Full, Zero & Full, Zero \\
\hline
\end{tabular}

monetary policy regimes.

As previously pointed out, the comparison of expected welfare equations (2.50)(2.51) and (2.82)-(2.83) reveals that welfare outcomes under full and zero transparency for the two policy regimes are identical:

$$
\begin{aligned}
& E\left[W_{R}^{\text {Full }}\right]=E\left[W_{D}^{\text {Full }}\right]=E\left[W^{\text {Full }}\right] \\
& E\left[W_{R}^{\text {Zero }}\right]=E\left[W_{D}^{\text {Zero }}\right]=E\left[W^{\text {Zero }}\right],
\end{aligned}
$$

thus, we drop the subscripts $R$ and $D$. Given this equivalence of outcomes under full and zero transparency, the following is true:

$$
\begin{array}{ccc}
E\left[W_{R}^{\text {Full }}\right] \leq E\left[W_{R}^{\text {Part }}\right] & \Longrightarrow & E\left[W^{\text {Full }}\right] \leq E\left[W_{R}^{\text {Part }}\right] \\
E\left[W_{R}^{\text {Zero }}\right] \leq E\left[W_{R}^{\text {Part }}\right] & \Longrightarrow & E\left[W^{\text {Zero }}\right] \leq E\left[W_{R}^{\text {Part }}\right],
\end{array}
$$

provided that $E\left[W_{R}^{\text {Part }}\right]$ is defined. Expected equilibrium welfare outcome under partial transparency $E\left[W_{R}^{\text {Part }}\right]$ exists when the optimal value of the variance of the additional noise $\sigma_{\eta}^{2}$ is positive. The latter is true when one of the six sets of conditions in (B.35a)-(B.40d) are satisfied. In other words, if a market is characterised by values of $\lambda, Q, r, \sigma_{\varepsilon}^{2}$ and $\sigma_{\phi}^{2}$ that satisfy any set of conditions from (B.35a)(B.40d), commitment to a rule in conjunction with partial transparency produce the best achievable outcome. For the remaining parameter combinations, the choice between commitment and discretion is arbitrary and the choice of an optimal disclosure regime parallels that under commitment. From Appendix B.4, full opacity is optimal if conditions (B.41a)-(B.41c) are satisfied and full transparency is optimal if one of the twelve sets of conditions in (B.42a)-(B.53c) are met. This brings us to Proposition 2.2.

Proposition 2.2: In the presence of information stickiness and if private agents aim to maximise a payoff function characterised by $\lambda \in[0,1]$, commitment to a policy rule performs at least as well as discretionary monetary policy and either full, 
partial or zero transparency is optimal, depending on parameter values.

From Proposition 2.2 it follows that a set of economies exists in which discretion performs as well as commitment to a rule. In our model the satisfactory performance of discretion under full and zero transparency is partly explained by the ability of market participants to infer the bank's response from the policy objective function with considerable accuracy. We assume that the private sector knows the policymaker's objective function with certainty, which is arguably a crude simplification. The accuracy of private agents' information about the bank's objective function is captured by what Geraats (2002) refers to as political transparency. Geraats gives an overview of the welfare effects of uncertainty about the policymaker's objectives that are explored in the relevant literature as well as the variety of formal approaches to modelling this uncertainty (Geraats, 2002, pp. F541-F547).

\subsubsection{M-S payoff function $(\lambda=0)$}

We now briefly revise Proposition 2.2 for the special case of the M-S payoff function. When $\lambda=0$, full transparency is always optimal under discretion and either full or partial transparency is optimal under commitment, depending on parameter values. In line with Proposition 2.2, comparison of welfare outcomes in (2.54)-(2.55) and (2.92) indicates that commitment performs better than discretion when partial transparency is optimal ${ }^{33}$. Based on the conditions for optimality of partial transparency under commitment in (2.53a)-(2.53b), commitment to a rule is strictly preferable to discretion when:

$$
\left\{\begin{array}{l}
0<Q<Q_{1} \\
0<\sigma_{\phi}^{2}<\sigma_{\phi 1}^{2},
\end{array}\right.
$$

where:

$$
\begin{aligned}
Q_{1} & \equiv \frac{r^{2}-r^{3}}{1+r+r^{2}-r^{3}} \\
\sigma_{\phi 1}^{2} & \equiv \frac{\sigma_{\varepsilon}^{2}}{1-(1-2 Q) r}\left\{\frac{(1-Q)(1-r) r \sqrt{Q[1-(1-Q)(2-r) r]}}{Q[1-(1-Q)(2-r) r]}-(1+r)\right\} .
\end{aligned}
$$

\subsubsection{J-L payoff function $(\lambda=1)$}

In case of the J-L payoff function, when $\lambda=1$, Proposition 2.2 simplifies to a condition on $r$, which can be expressed in terms of $Q, \sigma_{\varepsilon}^{2}$ and $\sigma_{\phi}^{2}$. Depending on parameter values, either full or zero transparency is optimal under discretion, whereas one of the three possible disclosure regimes is optimal under commitment. Comparison of

\footnotetext{
${ }^{33}$ See Appendix B.8 for a graphical representation of a numerical example.
} 
expected welfare expressions in (2.59)-(2.61) and (2.95)-(2.96) confirms that commitment outperforms discretion when partial transparency is optimal ${ }^{34}$. In case of both policy regimes we expressed the optimality conditions of different transparency regimes in terms of $r$. From (2.57)-(2.58) and (2.97), the critical values of $r$ used in our analysis are as follows:

$$
\begin{aligned}
& r_{1}=\frac{\left(\sigma_{\varepsilon}^{2}+\sigma_{\phi}^{2}\right) \sqrt{Q}}{(1+\sqrt{Q})\left(\sigma_{\varepsilon}^{2}+\sigma_{\phi}^{2} \sqrt{Q}\right)} \\
& r_{2}=\frac{1}{1+\sqrt{Q}} \\
& r_{3}=\frac{\sigma_{\varepsilon}^{2}+\sigma_{\phi}^{2}-\sqrt{\left(\sigma_{\varepsilon}^{2}+\sigma_{\phi}^{2}\right)\left(\sigma_{\varepsilon}^{2}+Q \sigma_{\phi}^{2}\right)}}{(1-Q) \sigma_{\phi}^{2}} .
\end{aligned}
$$

These critical values define the ranges for which either both regimes or only commitment to a rule is optimal. Given that the relationship between the critical values is:

$$
0<r_{1}<r_{3}<r_{2}<1
$$

the conditions for optimality of different transparency regimes in Table 2.1 and Table 2.2 can be combined in a manner demonstrated in Table 2.4.

Table 2.4: Conditions for optimal choice of policy regime

\begin{tabular}{|r|c|c|c|}
\hline Condition & $0<r \leq r_{1}$ & $r_{1}<r<r_{2}$ & $r_{2} \leq r<1$ \\
Transparency & Full & Partial & Zero \\
$\begin{array}{r}\text { Optimal Policy } \\
\text { Regime }\end{array}$ & $\begin{array}{c}\text { Either commitment } \\
\text { or discretion }\end{array}$ & Commitment & $\begin{array}{c}\text { Either commitment } \\
\text { or discretion }\end{array}$ \\
\hline
\end{tabular}

\subsection{Efficient Benchmark}

In the previous Section we evaluated the relative performance of commitment and discretion. We continue our analysis by assessing the efficiency of equilibrium outcomes that correspond to the two monetary policy regimes. As in the case of policy performance, we evaluate efficiency in terms of expected social welfare and in order to be able to do that we first derive an efficient benchmark ${ }^{35}$

\footnotetext{
${ }^{34}$ See Appendix B.9 for a numerical example and a graphical illustration of this result.

${ }^{35}$ Underlying assumptions for this scenario have already been discussed in Section 2.2.1.1.
} 
Following Angeletos and Pavan (2007a,b) and James and Lawler (2011, 2012a,b), the efficient case corresponds to the welfare outcome achieved through efficient use of information available to every agent. The information is used efficiently to inform actions of market participants and direct policy intervention. It should be noted that the collectively efficient use of information is still constrained by the way this information is allocated between agents and the CB. The assumptions about the allocation of information remain the same as in equilibrium cases, preserving the heterogeneity and stickiness. In order to derive the efficient outcome we make a sole additional assumption - we allow the policymaker to instruct market participants on how to use the different types of information available to them. The benevolent policymaker forms these instructions with social welfare in mind, which ensures maximum attainable efficiency of the expected welfare outcome, given the structure of the model.

\subsubsection{Efficient case without passive agents $(Q=0)$}

As before, we begin by looking at the simplified model without information stickiness, where $Q=0$. The $\mathrm{CB}$ is facing an optimisation problem of the following form:

$$
\max E[W]_{Q=0} \quad \text { w.r.t. } \kappa_{0}, \kappa_{1}, \kappa_{2}, \rho_{0}, \rho_{1}, \rho_{2}, \sigma_{\eta}^{2} \text {, }
$$

where the expression for expected welfare is take from (2.39):

$$
\begin{aligned}
E[W]_{Q=0}= & -\kappa_{1}^{2} \sigma_{\varepsilon}^{2}-(1-\lambda r)^{2}\left[\left(\kappa_{0}-\rho_{0}\right)^{2}+\left(\kappa_{1}+\kappa_{2}-1-\rho_{1}-\rho_{2}\right)^{2} \sigma_{\theta}^{2}+\right. \\
& \left.+\left(\kappa_{2}-\rho_{1}-\rho_{2}\right)^{2} \sigma_{\phi}^{2}+\left(\kappa_{2}-\rho_{2}\right)^{2} \sigma_{\eta}^{2}\right] .
\end{aligned}
$$

Maximising expected social welfare with respect to the policy parameters was the first step in deriving the equilibrium outcome under discretionary monetary policy in Section 2.4. Thus, we refer to expressions (2.66a)-(2.66c):

$$
\begin{aligned}
& \rho_{0}^{*}=\kappa_{0} \\
& \rho_{1}^{*}=\kappa_{1}-1 \\
& \rho_{2}^{*}=\kappa_{2},
\end{aligned}
$$

and substitute these values into (2.39). The substitution of (2.66a)-(2.66c) eliminates $\kappa_{0}, \kappa_{2}$ and $\sigma_{\eta}^{2}$. Differentiating the resulting expression with respect to $\kappa_{1}$, solving the corresponding first-order condition and using expressions (2.66a) and (2.66c), 
yields the following set of values for efficient weights and policy parameters:

$$
\begin{aligned}
& \tilde{\kappa}_{0}=\tilde{\rho}_{0} \\
& \tilde{\kappa}_{1}=\frac{(1-\lambda r)^{2} \sigma_{\phi}^{2}}{\sigma_{\varepsilon}^{2}+(1-\lambda r)^{2} \sigma_{\phi}^{2}} \\
& \tilde{\kappa}_{2}=\tilde{\rho}_{2},
\end{aligned}
$$

where ${ }^{\sim}$ denotes efficient value. Equations (2.129a) and (2.129c) indicate that there is an infinite number of combinations of efficient values for the action weights and policy parameters. Every combination yields the same level of expected welfare, obtained by substituting (2.129a)-(2.129c) into (2.39):

$$
E[\tilde{W}]_{Q=0}=-\frac{(1-\lambda r)^{2} \sigma_{\varepsilon}^{2} \sigma_{\phi}^{2}}{\sigma_{\varepsilon}^{2}+(1-\lambda r)^{2} \sigma_{\phi}^{2}} .
$$

In the case of the M-S payoff function $(\lambda=0)$, the efficient welfare outcome in (2.130) becomes equivalent to the result obtained by James and Lawler (2011), whereas in the J-L case $(\lambda=1)$ it is identical to the expression derived in James and Lawler (2012b).

The substitution of efficient values for $\kappa_{0}, \kappa_{1}$ and $\kappa_{2}$ into the welfare equation eliminates the variance of the additional noise $\sigma_{\eta}^{2}$ rendering the choice of a transparency regime redundant. Equation $\left(2.129 \mathrm{c}\right.$ ) and the coefficients on $\sigma_{\eta}^{2}$ in the expected welfare expression (2.39):

$$
-(1-\lambda r)^{2}\left(\kappa_{2}-\rho_{2}\right)^{2} \sigma_{\eta}^{2}
$$

show that, for any $0<\tilde{\kappa}_{2}$ and $0<\tilde{\sigma}_{\eta}^{2}$, it is optimal for the policymaker to fully offset any non-zero components of agents' actions that constitute an implicit response to $\eta^{36}$. Thus, the combinations of efficient values of $\kappa_{2}, \rho_{2}$ and $\sigma_{\eta}^{2}$, that can be grouped in the following way:

$$
\begin{aligned}
& \left(\tilde{\kappa}_{2}=\tilde{\rho}_{2} \neq 0, \sigma_{\eta}^{2}=0\right) \\
& \left(\tilde{\kappa}_{2}=\tilde{\rho}_{2} \neq 0,0<\sigma_{\eta}^{2}\right) \\
& \left(\tilde{\kappa}_{2}=\tilde{\rho}_{2}=0, \sigma_{\eta}^{2}=0\right) \\
& \left(\tilde{\kappa}_{2}=\tilde{\rho}_{2}=0,0<\sigma_{\eta}^{2}\right),
\end{aligned}
$$

all turn $-(1-\lambda r)^{2}\left(\kappa_{2}-\rho_{2}\right)^{2} \sigma_{\eta}^{2}$ to zero thereby neutralising the effect of transparency

\footnotetext{
${ }^{36}$ The response is referred to as implicit since active agents explicitly respond to the public signal through $\kappa_{2} y$, which can be decomposed into an $\kappa_{2} \theta$ and $\kappa_{2} \eta$
} 
policy on welfare.

We proceed by deriving the efficient outcome for intermediate degrees of information stickiness $(0<Q<1)$. In parallel to the equilibrium solution under discretion, we then attempt to isolate a single set of efficient parameters for the setting with no passive agents by setting $Q=0$.

\subsubsection{Efficient case with passive agents $(0<Q<1)$}

In the case of intermediate degrees of information stickiness $(0<Q<1)$, the policymaker aims to maximise expected welfare given by (B.20). Thus, the optimisation problem can be formulated as follows:

$$
\max E[W] \quad \text { w.r.t. } \quad \kappa_{0}, \kappa_{1}, \kappa_{2}, \rho_{0}, \rho_{1}, \rho_{2}, \sigma_{\eta}^{2} \text {. }
$$

Similarly to the $Q=0$ case, we refer to Section 2.4 for the optimal setting of policy, given by expressions $(2.73 \mathrm{a})-(2.73 \mathrm{c})$ :

$$
\begin{aligned}
& \rho_{0}^{*}=(1-Q) \kappa_{0} \\
& \rho_{1}^{*}=(1-Q) \kappa_{1}-1 \\
& \rho_{2}^{*}=(1-Q) \kappa_{2} .
\end{aligned}
$$

Having substituted (2.73a)-(2.73c) into (B.20), we differentiate the resulting expression with respect to $\kappa_{0}, \kappa_{1}$ and $\kappa_{2}$. Solving the first-order conditions yields the following values for efficient weights parameters:

$$
\begin{aligned}
& \tilde{\kappa}_{0}=0 \\
& \tilde{\kappa}_{1}=\frac{(1-\lambda r)^{2} \sigma_{\phi}^{2}}{\sigma_{\varepsilon}^{2}+[1-(1-Q)(2-\lambda r) \lambda r] \sigma_{\phi}^{2}+Q \sigma_{\eta}^{2}} \\
& \tilde{\kappa}_{2}=-\tilde{\kappa}_{1}
\end{aligned}
$$

Substituting (2.73a)-(2.73c) and (2.132a)-(2.132c) into (B.20) yields:

$$
E[\tilde{W}]=-\frac{(1-\lambda r)^{2} \sigma_{\phi}^{2}\left[\sigma_{\varepsilon}^{2}+Q\left(\sigma_{\phi}^{2}+\sigma_{\eta}^{2}\right)\right]}{\sigma_{\varepsilon}^{2}+[1-(1-Q)(2-\lambda r) \lambda r] \sigma_{\phi}^{2}+Q \sigma_{\eta}^{2}}
$$

which can be further maximised with respect to the variance of the additional noise $\sigma_{\eta}^{2}$. The first derivative of $(2.133)$ with respect to $\sigma_{\eta}^{2}$ is negative, hence, full trans- 
parency is optimal. With $\sigma_{\eta}^{2}=0$ expression (2.133) becomes:

$$
E\left[\tilde{W}^{\text {Full }}\right]=-\frac{(1-\lambda r)^{2} \sigma_{\phi}^{2}\left(\sigma_{\varepsilon}^{2}+Q \sigma_{\phi}^{2}\right)}{\sigma_{\varepsilon}^{2}+[1-(1-Q)(2-\lambda r) \lambda r] \sigma_{\phi}^{2}},
$$

which is the efficient welfare outcome in an economy with intermediate degrees of information stickiness. As far as the special cases of our model are concerned, if we use the M-S payoff function, (2.134) becomes:

$$
E\left[\tilde{W}^{\text {Full }}\right]_{\lambda=0}=-\frac{\sigma_{\phi}^{2}\left(\sigma_{\varepsilon}^{2}+Q \sigma_{\phi}^{2}\right)}{\sigma_{\varepsilon}^{2}+\sigma_{\phi}^{2}},
$$

and in the case of the J-L payoff function the efficient welfare outcome is:

$$
E\left[\tilde{W}^{F u l l}\right]_{\lambda=1}=-\frac{(1-r)^{2} \sigma_{\phi}^{2}\left(\sigma_{\varepsilon}^{2}+Q \sigma_{\phi}^{2}\right)}{\sigma_{\varepsilon}^{2}+[1-(1-Q)(2-r) r] \sigma_{\phi}^{2}}
$$

Expected welfare in efficient outcomes (2.134), (2.135) and (2.136) is increasing in the quality of information available to the policymaker $\left(\frac{1}{\sigma_{\phi}^{2}}\right)$ and the private sector agents $\left(\frac{1}{\sigma_{\varepsilon}^{2}}\right)$ as well as the proportion of active agents $(1-Q)$.

We now turn to the welfare outcomes for the two extreme cases of information stickiness. We first re-attempt to derive the efficient weights and policy parameters for the case of no information stickiness by setting $Q=0$ in expressions (2.132a)(2.132c), which together with (2.66a)-(2.66c) gives:

$$
\begin{aligned}
& \tilde{\kappa}_{0}=\tilde{\rho}_{0}=0 \\
& \tilde{\kappa}_{1}=\tilde{\rho}_{1}+1=\frac{(1-\lambda r)^{2} \sigma_{\phi}^{2}}{\sigma_{\varepsilon}^{2}+(1-\lambda r)^{2} \sigma_{\phi}^{2}} \\
& \tilde{\kappa}_{2}=\tilde{\rho}_{2}=-\tilde{\kappa}_{1}
\end{aligned}
$$

This method isolates a single set of weights and policy parameters, however, the choice of a transparency policy remains arbitrary. As far as the case of complete information stickiness $(Q=1)$ is concerned, the policymaker cannot influence the way individual agents use their information, since none of the agents in the private sector observe any signals $\left(\kappa_{0}=\kappa_{1}=\kappa_{2}=0\right)$. Thus, the policymaker only has control over the policy parameters $\rho_{0}$ and $\rho_{1}\left(\rho_{2}\right.$ and $\sigma_{\eta}^{2}$ not being choice variable due to $y$ being unobservable by the private sector). The optimisation problem faced by the policymaker is identical to that already discussed in Section 2.4.4, which implies 
that efficient policy parameters are equal to those given by (2.107a)-(2.107b):

$$
\begin{aligned}
& \tilde{\rho}_{0}=0 \\
& \tilde{\rho}_{1}=-1 .
\end{aligned}
$$

and the efficient welfare outcome is equivalent to that in (2.108):

$$
E[\tilde{W}]_{Q=1}=-(1-\lambda r)^{2} \sigma_{\phi}^{2}
$$

Given (2.139), the efficient outcomes for the M-S and J-L payoff functions are:

$$
\begin{aligned}
& E[\tilde{W}]_{\lambda=0, Q=1}=-\sigma_{\phi}^{2} \\
& E[\tilde{W}]_{\lambda=1, Q=1}=-(1-r)^{2} \sigma_{\phi}^{2},
\end{aligned}
$$

respectively.

\subsubsection{Efficiency of equilibria with optimal policy}

Having derived a set of efficient outcomes under various assumptions, we are now in the position to assess the efficiency of equilibria discussed in Section 2.5. To follow the pattern established in previous Sections we will discuss the efficiency of equilibria under three alternative assumptions about the degree of information stickiness:

1. no passive agents $(Q=0)$,

2. intermediate degrees of information stickiness $(0<Q<1)$, and

3. no active agents $(Q=1)$.

In addition to that, we will consider the efficiency of equilibria for the special cases of the individual agents' payoff functions - M-S and J-L type.

James and Lawler (2011, 2012b) find that appropriately designed policy intervention combined with optimal disclosure policy achieve social optimum. The authors evaluate equilibria in two models with no information stickiness and either the M-S payoff function (James and Lawler, 2011), or the J-L payoff function (James and Lawler, 2012b). We extend this result to hybrid payoff functions characterised by $0<\lambda<1$. Comparison of (2.43) and (2.130) reveals that optimal commitment policy in combination with zero transparency yields an efficient welfare outcome:

$$
E\left[W_{R}^{Z \text { ero }}\right]_{Q=0}=E[\tilde{W}]_{Q=0} .
$$


As discussed earlier, in the absence of passive agents discretionary policy yields an inferior welfare outcome compared to commitment to a rule. This implies that the expected welfare outcome under discretion is inefficient, which is confirmed by the comparison of expressions (2.70) and (2.130). The result in (2.142) constitutes Proposition 2.3.

Proposition 2.3: If all private agents are active and aim to maximise a payoff function characterised by $\lambda \in(0,1)$, commitment to a monetary policy rule in combination with zero transparency with respect to the $C B$ 's private signal achieve efficient use of information.

It is straightforward to show that the use of information in equilibrium is efficient. Substituting the optimal rule parameter (2.41) into the equilibrium weights (2.37a)$(2.37 \mathrm{c})$ and evaluating the resulting expressions in the limit as the variance of the additional noise $\sigma_{\eta}^{2}$ approaches infinity yields:

$$
\begin{aligned}
\kappa_{0}^{*} & =\rho_{0} \\
\kappa_{1}^{*} & =\frac{(1-\lambda r)^{2} \sigma_{\phi}^{2}}{\sigma_{\varepsilon}^{2}+(1-\lambda r)^{2} \sigma_{\phi}^{2}} \\
\kappa_{2}^{*} & =\rho_{2} .
\end{aligned}
$$

Equilibrium weights in (2.143a)-(2.143c) are equivalent to the efficient values given by (2.129a)-(2.129c). Thus, by committing to an optimally designed rule and publishing an infinitely uninformative signal the policymaker induces active agents to use the information available to them efficiently. As $\sigma_{\eta}^{2}$ approaches infinity, the optimal policy parameter in (2.41) becomes:

$$
\rho_{1}^{*}=-\frac{\sigma_{\varepsilon}^{2}}{\sigma_{\varepsilon}^{2}+(1-\lambda r)^{2} \sigma_{\phi}^{2}}
$$

which is equal to $\kappa_{1}^{*}-1$, where $\kappa_{1}^{*}$ is given by $(2.143 \mathrm{~b})$. The fact that the optimal policy parameter in (2.144) satisfies the efficiency criterium ${ }^{37}$ implies that the policymaker also utilises its private information efficiently.

In the case of intermediate degrees of information stickiness $(0<Q<1)$, we compare the efficient welfare outcome (2.134) against the three expected equilibrium welfare outcomes identified in Section 2.5. The equilibrium outcomes to be used correspond to optimal combinations of monetary policy regimes and degrees of central bank transparency. We perform the comparison for every set of optimality

\footnotetext{
${ }^{37}$ The criterium is given by the combination of $(2.66 \mathrm{~b})$ and $(2.129 \mathrm{~b})$.
} 
conditions given in Appendix B.4. Table 2.5 outlines the equilibrium outcomes and corresponding sets of conditions for which the efficiency assessment is made. Table 2.6 and Table 2.7 present the equilibrium outcomes and optimality conditions for the special cases of the M-S payoff function and the J-L payoff function, respectively.

Table 2.5: Welfare outcomes and optimality conditions (hybrid case)

\begin{tabular}{|c|c|c|c|}
\hline Policy Regime & Disclosure & Welfare Outcome & Optimality Conditions \\
Commitment/Discretion & Full & See (2.50) or (2.82) & See (B.42a)-(B.53c) \\
Commitment & Partial & Omitted & See (B.35a)-(B.40d) \\
Commitment/Discretion & Zero & See (2.51) or (2.83) & See (B.41a)-(B.41c) \\
\hline
\end{tabular}

Table 2.6: Welfare outcomes and optimality conditions (M-S case)

\begin{tabular}{|c|c|c|c|}
\hline Policy Regime & Disclosure & Welfare Outcome & Optimality Conditions \\
Commitment/Discretion & Full & See (2.54) or (2.92) & $\begin{array}{c}\text { Remaining parameter } \\
\text { value combinations }\end{array}$ \\
Commitment & Partial & See (2.55) & See (2.53a)-(2.53b) \\
\hline
\end{tabular}

Table 2.7: Welfare outcomes and optimality conditions (J-L case)

\begin{tabular}{|c|c|c|c|}
\hline Policy Regime & Disclosure & Welfare Outcome & Optimality Conditions \\
Commitment/Discretion & Full & See (2.59) or $(2.95)$ & See Table 2.4 \\
Commitment & Partial & See $(2.60)$ & See Table 2.4 \\
Commitment/Discretion & Zero & See (2.61) or $(2.96)$ & See Table 2.4 \\
\hline
\end{tabular}

The evaluation of expected equilibrium welfare outcomes reveals that the efficient outcome is strictly greater than any of the equilibrium outcomes in the case of both the M-S and the J-L payoff functions. The same is true about the hybrid payoff function, with the exception of all combinations of parameter values characterised 
by $\lambda=\frac{1}{2}$. This leads us to Proposition 2.4 .

Proposition 2.4: In the presence of information stickiness and if private agents aim to maximise a payoff function characterised by $\lambda \in\left[0, \frac{1}{2}\right) \cup\left(\frac{1}{2}, 1\right]$, optimal monetary policy in combination with optimal disclosure policy fail to achieve efficient use of information.

Hybrid payoff functions characterised by $\lambda=\frac{1}{2}$ being the exception from Proposition 2.4 is not surprising. As mentioned before ${ }^{38}$, when the weight $\lambda$ is equal to a half, the equilibrium degree of coordination inherent in the payoff function coincides with the collectively efficient degree of coordination. In other words, the incentive for individual agents to coordinate their actions with each other in equilibrium is at a collectively efficient level which induces the agents to use their information efficiently. It should be noted that setting $\lambda=\frac{1}{2}$ does not eliminate the need for optimal direct policy intervention nor an optimally chosen disclosure policy ${ }^{39}$, which enable the CB to achieve an efficient welfare outcome:

$$
E\left[W^{\text {Full }}\right]_{\lambda=\frac{1}{2}}=E\left[\tilde{W}^{\text {Full }}\right]_{\lambda=\frac{1}{2}}=-\frac{(2-r)^{2} \sigma_{\phi}^{2}\left(\sigma_{\varepsilon}^{2}+Q \sigma_{\phi}^{2}\right)}{4 \sigma_{\varepsilon}^{2}+\left[4-4(1-Q) r+(1-Q) r^{2}\right] \sigma_{\phi}^{2}},
$$

from either (2.50) or (2.82) and (2.134). Optimal policy is needed to address the information asymmetry that stems from intermediate levels of information stickiness. In the absence of information stickiness $(Q=0)$ as well as in the case of complete information stickiness $(Q=1)$, the expected equilibrium welfare outcome is collectively efficient on its own, without policy intervention.

We conclude this Section by briefly considering the case of complete information stickiness $(Q=1)$. As discussed above, the absence of active agents means that the $\mathrm{CB}$ only has control over the way its own private signal is used to decide on the appropriate instrument adjustment. This means that the derivations required to obtain the expected equilibrium outcome are the same as the ones performed to arrive at the efficient benchmark. Thus, the equilibrium outcome in (2.108) and the efficient outcome in (2.139) are identical:

$$
E[W]_{Q=1}=E[\tilde{W}]_{Q=1}
$$

implying that in the absence of active agents the policymaker is able to achieve the

\footnotetext{
${ }^{38}$ See expressions (2.18) and (2.19) as well as equation (B.17) in Appendix B.1.

${ }^{39}$ Conditions (B.49a)-(B.49b) imply that, when $\lambda=\frac{1}{2}$, the policymaker should exercise full transparency in combination with either commitment to a rule or discretionary monetary policy.
} 
first-best outcome.

\subsection{Summary table of Chapter 2 results}

Before concluding this Chapter with some final remarks, we present a summary of obtained results in Table 2.8. Model variations and special cases are defined in terms of: (i) the value of the degree of information stickiness $Q$, (ii) the value of the payoff parameter $\lambda$, and (iii) the monetary policy regime $(M P)$. Relevant interpretations of parameter values and notation are as follows:

1. information stickiness: no passive agents $(Q=0)$, intermediate degrees of information stickiness $(0<Q<1)$, and no active agents $(Q=1)$;

2. parameter $\lambda$ : M-S case $(\lambda=0)$, equilibrium degree coordination inefficiently high $\left(0 \leq \lambda<\frac{1}{2}\right)$, equilibrium degree of coordination collectively efficient $\left(\lambda=\frac{1}{2}\right)$, equilibrium degree of coordination inefficiently low $\left(\frac{1}{2}<\lambda \leq 1\right)$, and J-L case $(\lambda=1)$;

3. policy regime: commitment to a rule $(R)$, and discretionary instrument adjustment $(D)$;

4. central bank communication: full transparency or Full $\left(\sigma_{\eta}^{2}=0\right)$, partial transparency or Part $\left(0<\sigma_{\eta}^{2} \wedge\left|\sigma_{\eta}^{2}\right|<\infty\right)$; and full opacity/zero transparency or Zero $\left(\sigma_{\eta}^{2} \rightarrow \infty\right)$;

5. efficiency: comparison of the expected equilibrium social welfare outcome to a scenario in which all information in the economy is used in a collectively efficient way. 


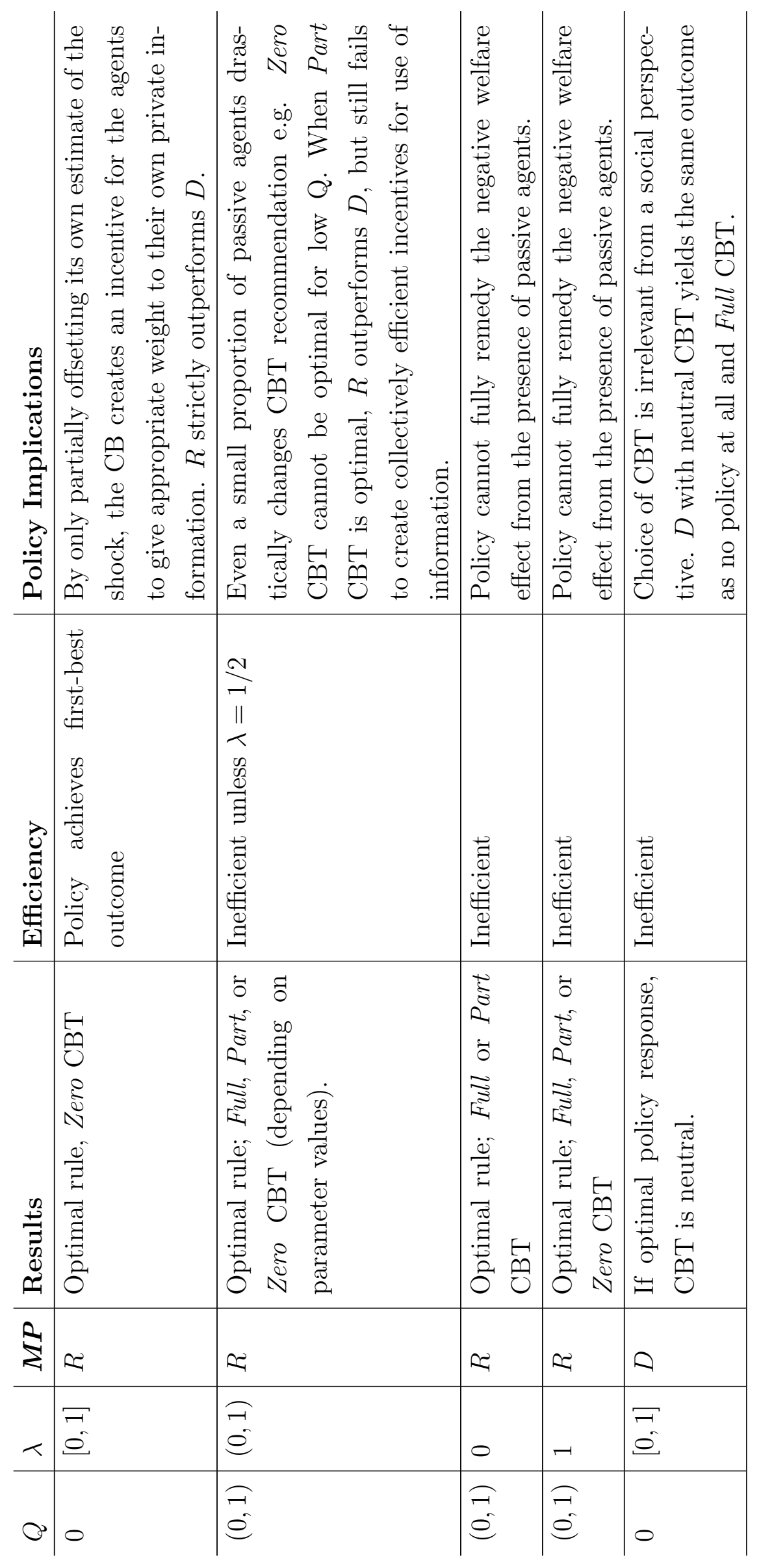




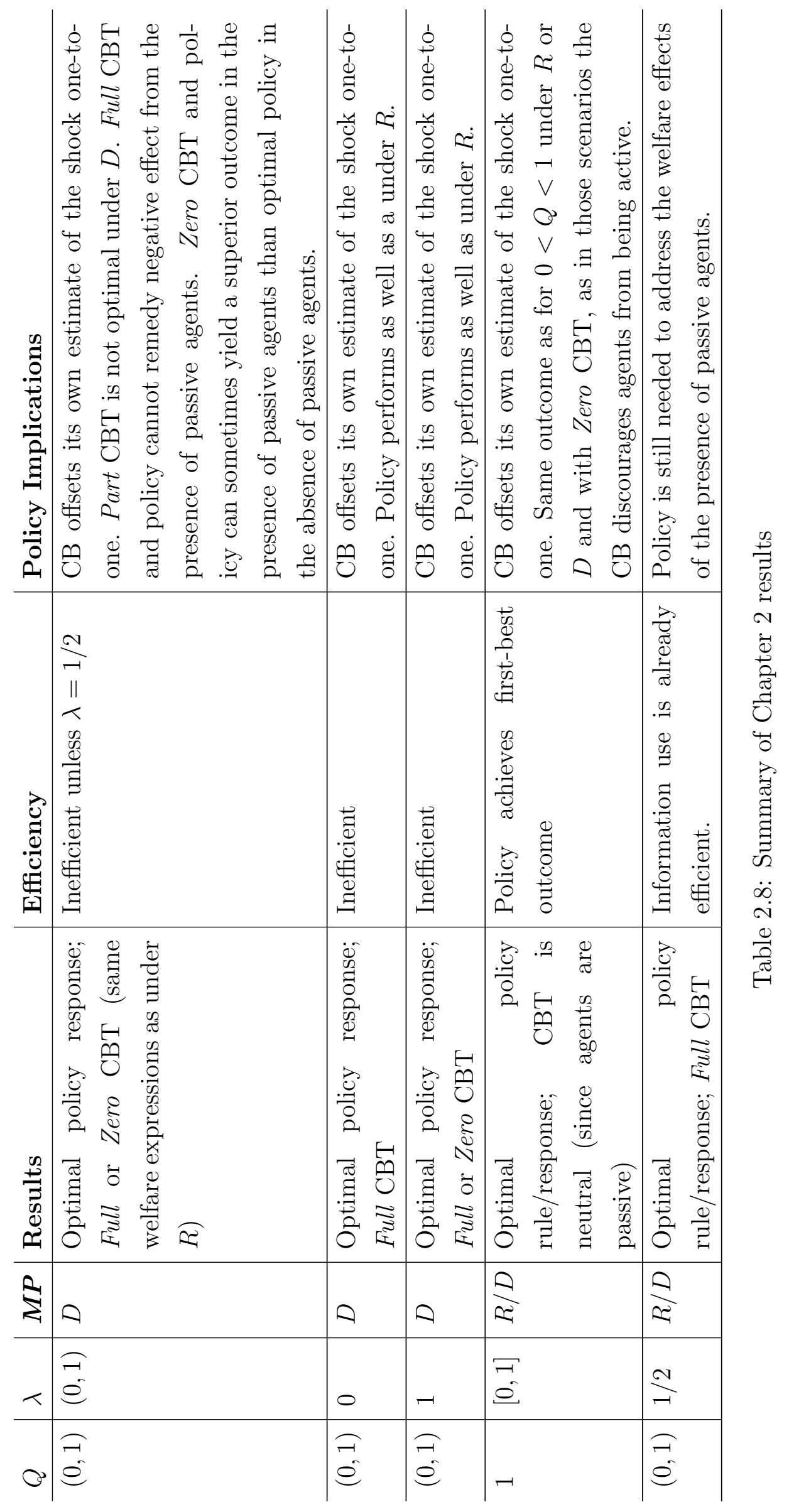




\subsection{Concluding remarks}

In the present Chapter we look at a modified and extended version of the original Morris-Shin framework. We follow James and Lawler (2011) in allowing the central bank to influence private sector behaviour directly through its instrument adjustment as well as indirectly through its communication policy; and we employ a mechanic from Hahn $(2010,2012)$ to incorporate information stickiness in the private sector. More specifically, we assume that a given proportion of population does not act upon new information (passive agents), while the remainder of the population takes all available information into account (active agents). We also construct a hybrid payoff function that encompasses a range of payoff functions with strategic complementarities, including what we refer to as Morris-Shin (M-S) and James-Lawler (J-L) payoffs as special cases. We then solve the model under the assumption of two policy regimes - commitment and discretion - and evaluate policy performance in terms of expected welfare outcomes.

The assumption of commitment in the present model translates into the CB announcing its policy rule prior to the private sector forming expectations. We begin the analysis by considering a special case scenario with no passive agents, i.e. with no information stickiness. From James and Lawler (2011, 2012b), we already know that, in the special cases of M-S and J-L payoff functions, it is optimal for the CB to practice full opacity in relation to its private information and instead use that information to partially offset the shock. We demonstrate that this result extends to our hybrid payoff function. We then re-introduce information stickiness and repeat the exercise. We show that the introduction of even a small proportion of passive agents leads to zero transparency in combination with an optimally designed rule no longer being unambiguously optimal. All three transparency regimes - full, partial, or zero - can be optimal depending on parameter values. Comparison with special cases presented in James and Lawler (2011, 2012b) reveals that the presence of passive agents has a detrimental effect on welfare. This negative effect cannot be fully offset by optimal policy design.

Under the discretionary policy regime, the $\mathrm{CB}$ reveals its objective function and the private sector forms its expectations of instrument adjustment, taking into account that the CB is aiming to maximise social welfare. Drawing a parallel with the commitment case, we begin by considering the scenario in which all agents are active. Similarly to James and Lawler (2011), we encounter multiplicity of equilibria. Each equilibrium corresponds to the same expected welfare outcome, which is independent of the CB's transparency tool. Moreover, the welfare outcome under 
discretion is the same as in a setup without direct policy intervention and with full transparency. We later successfully isolate a single equilibrium by solving the model under the assumption of sticky information and subsequently setting the proportion of passive agents to zero.

We proceed by considering discretionary policy in the presence of information stickiness. We find that, given optimal instrument adjustment, either full or zero transparency is optimal, and we present the sets of conditions that determine which disclosure regime is preferable. In the special case of the M-S payoff, only full transparency is optimal. We obtain two results in relation to the welfare effect of information stickiness that are of interest. Firstly, if full transparency is optimal in the presence of passive agents, then information stickiness has an unambiguously detrimental effect on welfare. Secondly, if zero transparency is optimal in the presence of passive agents and an additional set of conditions is satisfied, the considered economy outperforms a comparable economy with no passive agents.

Prior to characterising optimal policy design in the presence of sticky information, we consider another special case in which all agents are passive. When agents do not react to new information, it is unambiguously optimal for the CB to fully offset the shock, and this is true for both policy regimes. The expected welfare outcome is the same as in the scenarios with intermediate levels of information stickiness, zero transparency, and either commitment or discretion. Under zero transparency and optimal policy, active agents choose to ignore their private information and not to act, thereby, emulating an all-passive population.

We then turn to comparing how the two policy regimes perform in terms of expected social welfare. In the absence of information stickiness, James and Lawler (2011) find that commitment in combination with zero transparency strictly outperforms discretion in the special cases of the M-S and the J-L payoffs, and we extend their result to the hybrid payoff function. In the presence of information stickiness, we demonstrate that commitment performs at least as well as discretionary policy, which is a key result in the present Chapter. More specifically, commitment outperforms discretion when partial transparency is optimal and yields the same outcome when either full or zero transparency is optimal.

As a final step in this Chapter, we follow Angeletos and Pavan (2007a,b) and James and Lawler (2011, 2012a,b) in constructing an efficient benchmark. The benchmark case is characterised by efficient use of information, while assumptions of information asymmetry and stickiness are maintained. We use the derived bench- 
mark case to assess how optimal policy design performs in terms of efficiency. Extending the results in James and Lawler (2011, 2012b) to our hybrid payoff function, we show that, in the absence of information stickiness, the combination of optimal commitment and zero transparency achieves the first-best outcome. In the presence of information stickiness, optimal policy design, which includes optimal choice of a transparency regime, fails to achieve efficient use of information. There is a single exception to this result in our model, that relates to the way the hybrid payoff is constructed. Namely, when the perceived benefit of coordination at the level of an individual is the same as it would be perceived from a collective perspective, optimal monetary policy intervention and optimal disclosure policy produce the first-best welfare outcome.

To conclude, we highlight some of the policy implications of the key results obtained in this Chapter. Firstly, we demonstrated that, when the private sector is universally able to react to new information, the James and Lawler (2011) antitransparency result holds in a more general setting. This is an argument in favour of the CB not revealing its own economic forecasts and instead using that information to guide its instrument adjustments. However, the CB's ability to commit to a policy rule is a necessary condition for this argument to hold. Secondly, even the smallest proportion of economic agents who are either not able or unwilling to react to incoming information can reverse the anti-transparency result. Thirdly, intermediate levels of transparency, whereby the $\mathrm{CB}$ introduces vagueness into its forecast announcements, can potentially outperform full secrecy and complete honesty. This result is also predicated on the CB's ability to commit to an instrument adjustment rule. Finally, in an economy characterised by inefficient levels of coordination as well as information stickiness, the CB's policy response aims to simultaneously mitigate undesirable effects from two sources. We demonstrate that the CB is unable to fully offset both negative influences, but optimally-designed policy can at least partially remedy the situation. 


\section{Chapter 3}

\section{Costly Information}

\subsection{Introduction}

The present Chapter borrows certain elements of its structure, analytical methods as well as some of its core assumptions from Chapter 2. This is the case, primarily, since the general motivation behind the analysis performed here carries over from the previous Chapter. Namely, we continue exploring the way in which assumptions about information affect the interaction of central bank communication and monetary policy in the context of a coordination game. The discussion in the previous Chapter centres around considerations of information stickiness in the private sector and its effect on welfare. Here we explore a different factor that influences the informedness of economic agents and focus our attention on private information acquisition.

The original 'beauty-contest' game introduced by Morris and Shin (2002) as well as its prominent extensions ${ }^{1}$ analyse the social value of public announcements in the setting where individual agents are, on one hand, rewarded for matching their actions with economic fundamentals and, on the other hand, encouraged to align their actions with those of each other. Given these two objectives, agents make strategic choices on how to prioritise and use the information available to them. These choices also depend on the characteristics of available information, including its quality and accessibility. Hence, changing the assumptions about information has a direct effect on individual action strategies and, ultimately, the aggregate equilibrium outcome for the whole economy.

Morris and Shin distinguish between private and public information, where the former is only accessible by the respective individual agent, whereas the latter is

\footnotetext{
${ }^{1}$ Including Angeletos and Pavan (2007a), Cornand and Heinemann (2008), James and Lawler (2011).
} 
accessible by all agents. Although private information is heterogenous, in that it is different across agents, it is of homogeneous quality, as represented by a constant value of precision. Public information is observed by all agents without any difference in interpretation and its quality is also characterised by a constant value of precision. Morris and Shin treat both precisions as exogenous components of their framework ${ }^{2}$, endogenising solely the use of information. Variations of the original framework, such as the models analysed by Cornand and Heinemann (2008), James and Lawler (2011, 2012a,b) and Hahn (2012), include additional informational assumptions and explore the role of an explicit communication policy. Although these models endogenise either the accessibility or the quality of public announcements, the characteristics of private information observed by economic agents remain exogenous.

In this Chapter we introduce an additional strategic dimension into a modified Morris-Shin framework by endogenising private information acquisition. We first explore a scenario where agents have the option of purchasing private information of exogenous quality for a fixed price, we then follow Colombo and Femminis (2008) in assuming that the quality of private information is also a choice variable and information cost is linear in chosen accuracy ${ }^{3}$. Thus, individual agents in our model face two strategic choices - whether or not to acquire private information (and of what quality) and how to use this information in conjunction with the public information that is available. The central bank is also making two strategic decisions - designing an optimal monetary policy rule and making optimally transparent announcements. In modelling central bank choices we incorporate the approach employed in James and Lawler (2011) and allow the bank to conduct active stabilisation policy while adjusting the quality of publicly available information.

Once we introduce our model in Section 3.2, we split the remainder of the present Chapter into four parts. We consider a fixed cost structure in Sections 3.3 and 3.4 , and then we replace the fixed-fee assumption with a linear-cost approach from Colombo and Femminis (2008) in Sections 3.5 and 3.6. In contrast to the previous Chapter, here we abandon our all-encompassing hybrid payoff function and solve the model using re-formulated (to allow for introduction of a cost term) Morris-Shin and James-Lawler payoff functions separately. We construct an efficient benchmark scenario under the fixed and linear cost assumptions for each of the payoffs in Sections 3.3.1.5, 3.4.1.4, 3.5.1.3, and 3.6.1.3. We use the benchmark cases with efficient use

\footnotetext{
${ }^{2}$ The authors briefly consider the case where the quality of public information is optimally chosen by the public authorities, but transparency is not explicitly analysed as a policy tool.

${ }^{3}$ We have already outlined the key findings from Colombo and Femminis (2008) along with other references to costly information acquisition literature in Section 1.5.2.
} 
and acquisition of information to evaluate welfare effects of optimal policy design.

\subsection{Model}

In this Chapter we construct a set of coordination games. We borrow the original framework from Morris and Shin (2002) along with its alternative formulation from James and Lawler (2011). We then modify the games to incorporate two separate information cost structures, one of which is linear cost used by Colombo and Femminis (2008). Finally, we introduce direct monetary policy intervention in the way done by James and Lawler (2011). As a result, we derive equilibrium solutions to eight separate games, each corresponding to a combination of three assumptions from the following pairs:

- (i) fixed or linear cost structure;

- (ii) Morris-Shin or James-Lawler payoff function; and

- (iii) in the absence or in the presence of direct policy intervention.

We are unable to draw comparison between outcomes under different cost structures or payoff functions. Thus, we focus our analysis on the effects of direct policy intervention on welfare outcomes and information acquisition.

\subsubsection{Private Sector}

Private sector consists of an infinite number of individual economic agents, indexed by $i \in[0,1]$. Individual agents are atomistic in that agent $i$ 's strategy in isolation has no effect on the optimal choice of strategy by other agents or the central bank (CB).

\subsubsection{Payoff functions and coordination}

Individual agents aim to maximise their respective payoffs. In this Chapter we look at two payoff functions - Morris-Shin and James-Lawler ${ }^{4}$. In fact, we use re-formulated versions of the original counterparts of these two functions. We reformulate the Morris-Shin function in order to be able to add a cost component into it without affecting its core characteristics. Although the original James-Lawler specification for the payoff function allows for a cost component to be included with no unwanted consequences, we re-formulate both functions for the purpose of notation consistency. The rationale for re-formulation is explained in more detail in

\footnotetext{
${ }^{4}$ As mentioned in the previous Chapter, what we refer to as the 'James-Lawler' payoff function (prior to re-formulation) is an alternative payoff specification that also appears in Hellwig and Veldkamp (2009), and, thus is not exclusive to James and Lawler (2011, 2012b).
} 
Appendix C.1.

Under the assumption of Morris-Shin (M-S) payoff function, agent $i$ aims to maximise:

$$
u_{i}^{\mathrm{MS}}=-\left(a_{i}-\theta\right)^{2}-r\left(L_{i}-\bar{L}\right)-C_{i},
$$

where $a_{i}$ is agent $i$ 's action, $\theta$ is the state variable, $0<r$ is the coordination coefficient, $L_{i}=\int_{0}^{1}\left(a_{j}-a_{i}\right)^{2} d j, \bar{L}=\int_{0}^{1} L_{i} d i$, and $C_{i}$ is the information cost. The state variable $\theta$, also referred to here as the shock, reflects the economic fundamentals and is assumed to be uniformly distributed on the real line ${ }^{5}$. As in the original M-S payoff, $-\left(a_{i}-\theta\right)^{2}$ is the loss from imperfect alignment of agent $i$ 's action with the shock, and $-r\left(L_{i}-\bar{L}\right)$ is the 'beauty-contest' term, which is the loss from imperfect alignment between agent $i$ 's action and the action profile of the population. The coordination parameter $r$ can take on any positive value ${ }^{6}$ and reflects the relative importance of the coordination motive, as perceived by individual agents. Finally, the information cost $C_{i}$ will be discussed in more detail below.

The M-S payoff is characterised by strategic complementarity in agents' actions and, in Angeletos and Pavan's terms (Angeletos and Pavan, 2007a), an equilibrium degree of coordination (EDoC) that exceeds the collectively efficient degree of coordination $(\mathrm{CEDoC})^{7}$. In fact, the former is strictly positive, while the latter is zero, which implies that individual agents perceive there to be a benefit to aligning one's actions with others, whereas, at the level of the economy, coordination is neutral and has no benefit.

Turning now to the James-Lawler (J-L) payoff function, its re-formulated version is given by:

$$
u_{i}^{\mathrm{JL}}=-\left[a_{i}-\theta+r\left(a_{i}-\bar{a}\right)\right]^{2}-C_{i},
$$

where $a_{i}$ is agent $i$ 's action, $\theta$ is the state variable, $0<r$ is the coordination coefficient, $\bar{a}=\int_{0}^{1} a_{i} d i$ is the average action across the population, and $C_{i}$ is the information cost. The J-L payoff function implies that agent's actions are strategic complements, as in the M-S case. However, the two payoffs have different implication for coordination. The EDoC and CEDoC that correspond to the J-L payoff are both strictly positive, with the CEDoC exceeding the EDoC for all values of $0<r$. In other words, coordination has a benefit at both the individual and social levels. However, individual agents perceive the benefit to be smaller than what it

\footnotetext{
${ }^{5}$ The assumption of an improper prior greatly simplifies the analysis and improves the tractability of the model.

${ }^{6}$ As opposed to it being restricted to the $(0,1)$ interval in the original Morris-Shin framework.

${ }^{7}$ For derivation and brief analysis of the degrees of coordination, see Appendix C.2.
} 
is for the economy as whole. Unsurprisingly, this leads to important differences in how information is used in the model variation based on the M-S and J-L payoff functions.

\subsubsection{Information acquisition}

As far as endogenising information acquisition is concerned, we explore two different approaches. We first look at a discrete case with a fixed information cost, followed by model variations with a linear cost component.

Under the assumption of fixed cost, agent $i$ gets the choice of committing to a purchase of a private signal that reveals information about the state variable $\theta$. The private signal is given by:

$$
x_{i}=\theta+\varepsilon_{i}
$$

where $\varepsilon_{i}$ is an idiosyncratic noise that is distributed normally with a zero mean and precision $0<\beta$. The noise $\varepsilon_{i}$ introduces heterogeneity of information into outcomes where private information is acquired. In order to observe the private signal, a purchasing agent incurs an information cost given by:

$$
C_{i}=\nu_{i} p
$$

where $\nu_{i} \in\{0,1\}$ is the purchasing dummy variable, and $0<p$ is the price of information. The purchasing dummy $\nu_{i}$ is agent-specific and equals unity if agent $i$ decides to purchase $x_{i}$, or zero otherwise. The price $p$ is given exogenously, is fixed and the same for every agent. Thus, both the price and the quality of the purchased signals are exogenous and fixed.

Following Colombo and Femminis (2008), under the assumption of linear cost, instead of facing a discrete choice of whether or not to purchase a signal, agent $i$ gets to choose the quality of the purchased signal. Said signal is still private, noisy and given by:

$$
x_{i}=\theta+\varepsilon_{i},
$$

but this time the noise $\varepsilon_{i}$ is normally distributed with a zero mean and precision $\beta_{i}$. Thus agent $i$ gets to pick the individual precision of their private signal and incurs an information cost given by:

$$
C_{i}=\beta_{i} p
$$

where $0<p$ is the exogenously given price per unit of precision of $x_{i}$. Under these assumptions, the cost component is linear in $\beta_{i}$ and the choice of $\beta_{i}$ is endogenous to agent $i$ 's strategy. Any positive value of $\beta_{i}$ corresponds to purchasing a private 
signal, whereas any non-positive value is considered to be equivalent to not purchasing a signal at all.

In both cases we identify conditions for the existence of two types of Nash equilibria: (i) purchasing Nash equilibria or $P-N E$ are characterised by all agents acquiring an informative private signal, whereas (ii) non-purchasing Nash equilibria or $N$ - $N E$ are characterised by a complete absence of private information acquisition. In what follows, the term information acquisition, apart from its direct primary meaning as a process, is used to denote algebraic characteristics of information acquisition critical values of information price $p$ (in both fixed and linear cost cases) as well as the optimally chosen value of private signal precision $\beta_{i}$ (in linear cost case only).

\subsubsection{Central Bank}

Another player in the game is the central bank (CB). In making our assumptions about CB's actions, motives and information set, we closely follow James and Lawler (2011). The CB is a benevolent agent in that its only aim is to maximise social welfare. In its pursuit of that single aim, the CB has at its disposal two policy tools - central bank transparency (CBT) and direct policy intervention.

\subsubsection{Central bank transparency (CBT)}

The CB suffers from information asymmetry in that it cannot observe private sector signals when such signals are indeed purchased. However, the CB has access to its own private information, given in the form of a noisy signal about the state of fundamentals:

$$
z=\theta+\phi
$$

where the noise term $\phi$ is normally distributed with a zero mean and precision $\gamma$. We assume that the procurement or production of the signal $z$ comes at no cost to either the CB or the economy. Colombo and Femminis (2008) introduce a linear social cost of acquiring $z$ and give a social planner the power to choose precision $\gamma$, with $z$ automatically being made public. They find that in equilibrium only one type of information acquisition takes place - private or public - and which one depends on the relative price per unit of the two information sources. Here we focus on just private information acquisition along with its inefficiencies and assess CB's ability to rectify the latter, given a signal it acquires at no cost.

The CB commits to publishing a noisy version of its private signal:

$$
y=z+\eta=\theta+\phi+\eta,
$$


where the additional noise $\eta$ is distributed normally with a zero mean and precision $\frac{\alpha \gamma}{\gamma-\alpha}$. Thus, the precision of $\phi+\eta$ is equal to just $\alpha$. We refer to $\alpha$ as the CBT instrument, in that the $\mathrm{CB}$ chooses the quality of the public signal $y$ subject to the constraint $\alpha \leq \gamma$. The CB commits to its chosen value of $\alpha$ and makes it public knowledge prior to individual agents deciding on their strategies. We define three CBT regimes in terms of the chosen value of $\alpha$ as follows:

- full CBT corresponds to $0<\alpha=\gamma$ or $y=z$,

- intermediate CBT corresponds to $0<\alpha<\gamma$, and

- zero CBT corresponds to $\alpha=0$,

the latter being equivalent to there being no public information about $\theta$ available at all $(y=0)$. As an instrument, CBT has an indirect influence on information use and acquisition in the private sector. By varying $\alpha$, the $\mathrm{CB}$ varies the relative value of private information, as perceived by individual agents. In fact, in the eyes of agent $i$, signals $x_{i}$ and $y$ are strategic substitutes, which is explored in more detail in our equilibrium analysis below.

\subsubsection{Direct policy intervention}

The other tool in CB's policy kit is a monetary policy instrument that can be used to directly offset the shock represented by $\theta$. We assume that the CB is able to credibly commit to an instrument-adjustment rule of the form:

$$
g=\rho z
$$

where $\rho$ is the policy parameter. As with CBT, the optimal value of the policy parameter $\rho$ is chosen so as to maximise social welfare. Once it is chosen, the CB announces it to the private sector before any decision-making about information use and acquisition take place.

Direct policy intervention influences the outcome of the game through two channels. On the one hand, instrument adjustment $g$ enters the payoffs in (3.1) and (3.2) directly, as the term direct policy intervention suggests:

$$
\begin{aligned}
& u_{i}^{\mathrm{MS}}=-\left(a_{i}-\theta-g\right)^{2}-r\left(L_{i}-\bar{L}\right)-C_{i} \\
& u_{i}^{\mathrm{JL}}=-\left[a_{i}-\theta-g+r\left(a_{i}-\bar{a}\right)\right]^{2}-C_{i} .
\end{aligned}
$$


Instrument adjustment $g$ offsets the shock $\theta$ to the extent measured by $\rho$ and subject to a forecast error that arises from the fact that CB's private signal is of limited quality (precision $\gamma$ is finite). On the other hand, instrument adjustment $g$ enters the optimisation problem facing individual agents, which results in it having an indirect impact on information acquisition and use.

In this Chapter we solve the four model variations with and without direct policy intervention. We then focus our attention on the effect that the presence of policy has on private information acquisition, in particular.

\subsubsection{Game form}

The model in this Chapter is a sequential non-cooperative game with the final stage being a simultaneous-move subgame. The sequence of events in the model is as follows:

1. the $\mathrm{CB}$ announces the CBT parameter $\alpha$ and the policy parameter $\rho$ and credibly commits to them;

2. individual agents privately make a decision about information acquisition $\left(\nu_{i}\right.$ or $\beta_{i}$ ) and use;

3. simultaneous events: the $\mathrm{CB}$ observes $z$, reveals $y$, forms a forecast of $\theta$, and adjusts its instrument by amount $g$, while agent $i$ observes $x_{i}$ and $y$, forms a forecast of $\theta$ and $z$, and chooses action $a_{i}$; and,

4. finally, the shock $\theta$ is realised, individual payoffs and welfare are determined.

It should be noted that, because the decisions taken in step 2 are private and do not inform the decisions of others, step 2 can be seen as taking place within the simultaneous-move game in step 3. However, separating this element of decisionmaking into a stand-alone step improves clarity and highlights the fact that the value of $\nu_{i}$ (or $\beta_{i}$ ) has to be chosen prior to observing $x_{i}$, since the realisation of $x_{i}$ crucially depends on $\nu_{i}$ (or $\beta_{i}$ ).

Since this is a sequential game, the solution method for this model will involve backward induction. Due to the fact that the CB announces optimally designed policy which then enters into individual agents' decision-making, the CB can be seen as a Stackelberg leader with agents being the followers ${ }^{8}$. However, this is where the parallel ends, because in the present model agents and the CB are not engaged in competition with each other.

\footnotetext{
${ }^{8}$ These terms refer to the Stackelberg leadership model (Von Stackelberg, 2010).
} 


\subsubsection{Information use}

Here we characterise information use by performing derivation steps that are common for all model variations. We begin with a setup that includes direct policy intervention $(\rho \neq 0)$ and a linear information cost component $\left(C_{i}=\beta_{i} p\right)$, then, through simplification, we arrive at information use expressions that correspond to other model variations. We begin by assuming that agent $i$ 's action can be represented as a linear combination of observed signals. When all agents are purchasing a private signal, agent $i$ 's action is:

$$
a_{i}^{P}=\kappa_{1 i}^{P} x_{i}+\kappa_{2 i}^{P} y
$$

where superscript $P$ stands for purchasing, and $\kappa_{1 i}^{P}$ and $\kappa_{2 i}^{P}$ are best response weights. Whereas, when private information acquisition is not taking place, agent $i$ 's action is:

$$
a_{i}^{N}=\kappa_{2 i}^{N} y
$$

where superscript $N$ stands for non-purchasing, and $\kappa_{2 i}^{N}$ is the best response coefficient. In all further discussions, we assume that the term information use in algebraic terms corresponds to values of best response weights $\kappa_{1 i}^{P}, \kappa_{2 i}^{P}$, and $\kappa_{2 i}^{N}$.

Having identified the expression for agent $i$ 's action in its general form, we can obtain the population action profile by integrating (3.12) and (3.13) over $i \in[0,1]$. Following Colombo and Femminis (2008), we focus on equilibrium solutions that are symmetric in how private sector agents use available information. This implies that agent $i$ knows that all other agents $(j \neq i)$ follow identical strategy in that they choose the same best $\operatorname{responses}^{9}\left(\kappa_{1 j}^{P}=\kappa_{1}^{P}, \kappa_{2 j}^{P}=\kappa_{2}^{P}\right.$, and $\left.\kappa_{2 j}^{N}=\kappa_{2}^{N}\right)$ :

$$
\begin{aligned}
& a_{j}^{P}=\kappa_{1}^{P} x_{j}+\kappa_{2}^{P} y \\
& a_{j}^{N}=\kappa_{2}^{N} y .
\end{aligned}
$$

Integration of $a_{j}^{P}$ over $j \in[0,1]$ yields the average action when all agents are purchasing:

$$
\bar{a}^{P}=\kappa_{1}^{P} \theta+\kappa_{2}^{P} y
$$

where we made use of $\int_{0}^{1} \varepsilon_{j} d j=0$. When there is no private information acquisition, average action is given by:

$$
\bar{a}^{N}=\kappa_{2}^{N} y
$$

\footnotetext{
${ }^{9}$ Identical best responses imply that the equilibrium solutions are also symmetric in information acquisition, thus, agent $i$ knows that all other agents $(j \neq i)$ choose the same signal precision $\left(\beta_{j}=\beta\right)$.
} 


\subsubsection{Private sector expectations}

In order to proceed with the derivation of best-response weights, we first need to discuss agent $i$ 's conditional expectations of the state variable and CB's private signal, taking into account the observed signals. We use $E_{i}[\cdot]$ to denote expectations conditional on agent $i$ 's information set.

The expression for conditional expectation of $\theta$ is constructed in such a way so as to minimise the mean squared error (MSE) of $E_{i}[\theta]$ as an estimator of $\theta^{10}$. Same principle applies to the expectation of CB's signal $z$. When an agent observes two signals, expectations are given by:

$$
\begin{aligned}
& E_{i}\left[\theta \mid x_{i}, y\right]=\frac{\beta_{i} x_{i}+\alpha y}{\alpha+\beta_{i}}, \\
& E_{i}\left[z \mid x_{i}, y\right]=\frac{(\gamma-\alpha) \beta_{i} x_{i}+\alpha\left(\beta_{i}+\gamma\right) y}{\left(\alpha+\beta_{i}\right) \gamma} .
\end{aligned}
$$

When an agent observes only the public signal, expectations become:

$$
E_{i}[\theta \mid y]=E_{i}[z \mid y]=y
$$

Finally, when an agent observes no signals at all (no information acquisition takes place and the $\mathrm{CB}$ commits to a zero CBT regime), the conditional expectations are equivalent to the unconditional expectations and, thus, are equal to zero.

\subsubsection{Purchasing agents}

We only need to perform derivations for one payoff function, so we choose to do so for the M-S payoff. When all agents are purchasing private information, agent $i$ 's payoff in (3.1) becomes:

$$
u_{i}^{P}=-\left(a_{i}^{P}-\theta-g\right)^{2}-r\left(L_{i}^{P}-\bar{L}^{P}\right)-C_{i},
$$

where $L_{i}^{P}=\int_{0}^{1}\left(a_{j}^{P}-a_{i}^{P}\right)^{2} d j$ and $\bar{L}^{P}=\int_{0}^{1} L_{i}^{P} d i$. Using backward induction, we first assume that agent $i$ has already chosen signal precision $\beta_{i}$ and is now maximising conditional expectation of the payoff in (3.21) with respect to $a_{i}^{P}$. Optimisation yields the following best response:

$$
a_{i}^{P}=\frac{1}{1+r}\left(E_{i}\left[\theta \mid x_{i}, y\right]+E_{i}[g]\right)+\frac{r}{1+r} E_{i}\left[\bar{a}^{P}\right]
$$

\footnotetext{
${ }^{10}$ The method was already demonstrated in full in the Appendix of the previous Chapter, thus, here we merely take the already derived expressions and present them in terms of signal precisions, rather than variances.
} 
where $E_{i}[g]=\rho E_{i}\left[z \mid x_{i}, y\right]$ and:

$$
E_{i}\left[\bar{a}^{P}\right]=\kappa_{1}^{P} E_{i}\left[\theta \mid x_{i}, y\right]+\kappa_{2}^{P} y
$$

from (3.16). We, thus, have two representations of optimal action given by (3.12) and (3.22). We substitute $E_{i}[\theta]$ from (3.18), $E_{i}[z]$ from (3.19), and $E_{i}\left[\bar{a}^{P}\right]$ from (3.23) into (3.22) and compare the coefficients on the two expressions for optimal action to find the following optimal weights:

$$
\begin{aligned}
\kappa_{1 i}^{P} & =\frac{\left[\left(1+r \kappa_{1}^{P}+\rho\right) \gamma-\alpha \rho\right] \beta_{i}}{(1+r)\left(\alpha+\beta_{i}\right) \gamma} \\
\kappa_{2 i}^{P} & =1+\rho-\kappa_{1 i}^{P} .
\end{aligned}
$$

Since all agents other than agent $i$ follow an identical strategy, we can work out the values of weights $\kappa_{1}^{P}$ and $\kappa_{2}^{P}$ by substituting $\kappa_{1 i}^{P}=\kappa_{1}^{P}, \kappa_{2 i}^{P}=\kappa_{2}^{P}$ into the left-hand side and $\beta_{j}=\beta$ into the right-hand side of (3.24)-(3.25). Solving for $\kappa_{1}^{P}$ and $\kappa_{2}^{P}$ yields:

$$
\begin{aligned}
& \kappa_{1}^{P}=\frac{[(1+\rho) \gamma-\alpha \rho] \beta}{[(1+r) \alpha+\beta] \gamma} \\
& \kappa_{2}^{P}=1+\rho-\kappa_{1}^{P} .
\end{aligned}
$$

Since agent $i$ expects a common value for $\beta$ across other agents and, hence, will expect $\bar{a}$ to be characterised by the above values of $\kappa_{1}^{P}$ and $\kappa_{2}^{P}$, substituting said values in agent's own individually optimal responses to $x_{i}$ and $y$ yields:

$$
\begin{aligned}
\kappa_{1 i}^{P} & =\frac{[(1+\rho) \gamma-\alpha \rho](\alpha+\beta) \beta_{i}}{[(1+r) \alpha+\beta]\left(\alpha+\beta_{i}\right) \gamma} \\
\kappa_{2 i}^{P} & =1+\rho-\kappa_{1 i}^{P}
\end{aligned}
$$

Expressions (3.26)-(3.27) and (3.28)-(3.29) characterise information use by the population and agent $i$, respectively, in the case with a linear cost component and direct policy intervention.

Without policy, agent $i$ 's optimal weights become:

$$
\begin{aligned}
& \left.\kappa_{1 i}^{P}\right|_{\rho=0}=\frac{(\alpha+\beta) \beta_{i}}{[(1+r) \alpha+\beta]\left(\alpha+\beta_{i}\right)} \\
& \left.\kappa_{2 i}^{P}\right|_{\rho=0}=1-\kappa_{1 i}^{P}
\end{aligned}
$$


and the best-responses of other agents $(j \neq i)$ are characterised by:

$$
\begin{aligned}
& \left.\kappa_{1}^{P}\right|_{\rho=0}=\frac{\beta}{(1+r) \alpha+\beta} \\
& \left.\kappa_{2}^{P}\right|_{\rho=0}=1-\kappa_{1}^{P} .
\end{aligned}
$$

The four expressions above are obtained simply by substituting $\rho=0$ into (3.28)(3.29) and(3.26)-(3.27).

Under the assumption of a fixed information $\operatorname{cost}\left(C_{i}=\nu_{i} p\right.$ and $\left.\nu_{i}=1\right)$, agentspecific weights $\kappa_{1 i}^{P}$ and $\kappa_{2 i}^{P}$ become irrelevant, as $\beta$ is exogenous. Thus, information use is characterised by (3.26)-(3.27) in the presence of direct policy intervention and by (3.32)-(3.33) in absence of it. Finally, the above derivation method and expressions for $\kappa_{1 i}^{P}, \kappa_{2 i}^{P}, \kappa_{1}^{P}$, and $\kappa_{2}^{P}$ equally apply to model variations with the J-L payoff function.

\subsubsection{Non-purchasing agents}

We now repeat the above procedure for identifying best-response weights under the assumption that there is no private information acquisition. Agent $i$ 's payoff in (3.1) becomes:

$$
u_{i}^{N}=-\left(a_{i}^{N}-\theta-g\right)^{2}-r\left(L_{i}^{N}-\bar{L}^{N}\right),
$$

where $L_{i}^{N}=\int_{0}^{1}\left(a_{j}^{N}-a_{i}^{N}\right)^{2} d j, \bar{L}^{N}=\int_{0}^{1} L_{i}^{N} d i$, and information cost is zero. Agent $i$ is maximising conditional expectation of the payoff in (3.34) with respect to $a_{i}^{N}$, and the optimisation yields the following best response:

$$
a_{i}^{N}=\frac{1}{1+r}\left(E_{i}[\theta \mid y]+E_{i}[g]\right)+\frac{r}{1+r} E_{i}\left[\bar{a}^{N}\right],
$$

where $E_{i}[g]=\rho E_{i}[z \mid y]$ and $E_{i}\left[\bar{a}^{N}\right]=\bar{a}^{N}$. We, thus, have two representations of optimal action given by (3.13) and (3.35). We substitute $E_{i}[\theta]$ and $E_{i}[z]$ from (3.20), and $E_{i}\left[\bar{a}^{N}\right]$ from (3.17) into (3.35) and compare the coefficients on the two expressions for optimal action. As a result, we find the following best-response weight:

$$
\kappa_{2 i}^{N}=\kappa_{2}^{N}=1+\rho,
$$

which does not depend on any agent-specific variables and, thus, is the same for all agents. Setting $\rho=0$ yields the optimal weight under the assumption of there being no direct policy intervention:

$$
\left.\kappa_{2}^{N}\right|_{\rho=0}=1
$$


where a $\kappa_{2}^{N}$ equal to unity implies that agent $i$ 's action is simply equal to the public signal $y$. Optimal weight values (3.36)-(3.37) equally apply to model variations with fixed and linear cost components, as well both payoff functions.

\subsubsection{Social welfare}

In this model social welfare is merely the aggregate of individual payoffs across the population. Thus, from (3.1) and (3.2), ex post social welfare for the two payoff functions is given by:

$$
\begin{aligned}
& W^{\mathrm{MS}}=-\int_{0}^{1}\left(a_{i}-\theta-g\right)^{2} d i-\int_{0}^{1} C_{i} d i \\
& W^{\mathrm{JL}}=-\int_{0}^{1}\left[a_{i}-\theta-g+r\left(a_{i}-\bar{a}\right)\right]^{2} d i-\int_{0}^{1} C_{i} d i, .
\end{aligned}
$$

In line with the characteristics of the original Morris-Shin framework, social welfare expression aggregated from the M-S payoff does not contain a 'beauty contest' term, as the latter turns to zero when integrated over $i \in[0,1]$.

\subsubsection{Expected social welfare}

For its strategic policy decisions the CB uses the ex ante social welfare. In order to obtain expressions for expected social welfare, we substitute for $a_{i}, g$, and $\bar{a}$ into (3.38) and (3.39) as well as use the definitions of signals $x_{i}, z$, and $y$ from (3.5), (3.7), and (3.8), respectively. We then take unconditional expectations of the resulting expressions. When all agents are purchasing, we use (3.14) and (3.16) to substitute for $a_{i}^{P}$ and $\bar{a}^{P}$, respectively, which yields:

$$
\begin{aligned}
& E\left[W^{\mathrm{MS}-\mathrm{P}}\right]=-\frac{\left(\kappa_{1}^{P}\right)^{2}}{\beta}-\left[\frac{\left(\kappa_{2}^{P}\right)^{2}}{\alpha}-\frac{\left(2 \kappa_{2}-\rho\right) \rho}{\gamma}\right]-\int_{0}^{1} C_{i} d i \\
& E\left[W^{\mathrm{JL}-\mathrm{P}}\right]=-\frac{(1+r)^{2}\left(\kappa_{1}^{P}\right)^{2}}{\beta}-\left[\frac{\left(\kappa_{2}^{P}\right)^{2}}{\alpha}-\frac{\left(2 \kappa_{2}-\rho\right) \rho}{\gamma}\right]-\int_{0}^{1} C_{i} d i .
\end{aligned}
$$

Above expressions merely ${ }^{11}$ lose the third term that includes $\rho$ when there is no direct policy intervention:

$$
\begin{aligned}
& E\left[W^{\mathrm{MS}-\mathrm{P}}\right]_{\rho=0}=-\frac{\left(\kappa_{1}^{P}\right)^{2}}{\beta}-\frac{\left(\kappa_{2}^{P}\right)^{2}}{\alpha}-\int_{0}^{1} C_{i} d i, \\
& E\left[W^{\mathrm{JL}-\mathrm{P}}\right]_{\rho=0}=-\frac{(1+r)^{2}\left(\kappa_{1}^{P}\right)^{2}}{\beta}-\frac{\left(\kappa_{2}^{P}\right)^{2}}{\alpha}-\int_{0}^{1} C_{i} d i .
\end{aligned}
$$

\footnotetext{
${ }^{11}$ There is also the implied difference in the values of best-response terms $\kappa_{1}^{P}$ and $\kappa_{2}^{P}$, compared to the case that includes policy.
} 
Substituting the N-NE expressions for $a_{i}^{N}=\bar{a}^{N}$ from (3.15) and (3.17) as well as $\kappa_{2}^{N}$ from (3.36)-(3.37) into (3.38)-(3.39) and taking expectations gives us the N-NE expected welfare:

$$
\begin{aligned}
& E\left[W^{N}\right]=-\frac{(1+\rho)^{2}}{\alpha}+\frac{(2+\rho) \rho}{\gamma} \\
& E\left[W^{N}\right]_{\rho=0}=-\frac{1}{\alpha} .
\end{aligned}
$$

The expected welfare outcomes in N-NE are the same for the two payoff functions.

The discussion in Angeletos and Pavan (2007a) offers a useful approach to analysing social welfare effects and considerations. Angeletos and Pavan introduce the terms volatility and dispersion in the context of a wider set of models. Volatility is the common variation in actions and in our model refers to $\operatorname{Var}[\bar{a}-\theta]$, whereas dispersion is the idiosyncratic variation in actions and corresponds to $\operatorname{Var}\left[a_{i}-\bar{a}\right]$. Using these two definitions, expected welfare can be conveniently presented in terms of three components: loss from dispersion, loss from volatility, and information acquisition cost. In Appendix C.3 we demonstrate that P-NE expected welfare in the M-S case corresponds to:

$$
E\left[W^{\mathrm{MS}-\mathrm{P}}\right]=- \text { Dispersion }^{P}-\left[\text { Volatility }^{P}\right]-\text { Information Cost }
$$

where loss from dispersion is the first term in (3.40) and (3.42) and loss from volatility is the second term (term in square brackets). Similarly, the P-NE expected welfare in the J-L case, given by (3.41) and (3.43), can be represented as:

$$
E\left[W^{\mathrm{JL}-\mathrm{P}}\right]=-(1+r)^{2} \cdot \text { Dispersion }^{P}-\left[\text { Volatility }^{P}\right]-\text { Information Cost },
$$

where the dispersion term is amplified by $(1+r)^{2}$, which reflects the non-neutrality of coordination at the aggregate level. Finally, the N-NE expected welfare has neither dispersion nor cost component:

$$
E\left[W^{N}\right]=- \text { Volatility }^{N}
$$

It is worth noting that instrument adjustment $g$ directly enters the expression for volatility $^{12}: \operatorname{Var}[\bar{a}-\theta-g]$. As a result, the policy parameter $\rho$ explicitly enters the volatility term in (3.40), (3.41) and (3.44). However, instrument adjustment also

\footnotetext{
${ }^{12}$ As discussed in Appendix C.3, $\theta$ in $\operatorname{Var}[\bar{a}-\theta]$ stands for what the average action would be under the assumption of perfect information. Thus, the introduction of policy directly into the volatility expression can be interpreted as the CB using its private information to bring the economy closer to a perfect information benchmark.
} 
has an indirect effect on the dispersion and volatility terms through the presence of parameter $\rho$ in the best-response weights $\kappa_{1}^{P}$ and $\kappa_{2}^{P}$.

\subsubsection{Policy and CBT in N-NE}

The N-NE welfare outcomes, given in (3.44) and (3.45), do not depend on the cost structure or the payoff function type. Thus, we can discuss optimal policy and CBT setting in N-NE before going into any more detail with specific model variations.

In the absence of policy, agents' actions are just the public signal $y$ and expected welfare outcome is given by (3.45):

$$
E\left[W^{N}\right]_{\rho=0}=-\frac{1}{\alpha}
$$

Coordination of actions is perfect and there is no idiosyncratic action dispersion. Private information acquisition is not taking place, hence, there is no information cost either. The only loss component is volatility loss, which means that any improvement in the quality of public information is welfare-enhancing, as it allows agents to align their actions more closely with the fundamentals. Expected welfare is strictly increasing in $\alpha$, thus it is optimal for the CB to commit to full CBT and set $\alpha=\gamma$. The N-NE expected welfare outcome is then:

$$
E\left[W^{N}\right]_{\rho=0, \alpha=\gamma}=-\frac{1}{\gamma}
$$

which is equal to negative variance of CB's forecast errors about the shock.

In the presence of direct policy intervention, agents' actions are equal to $(1+\rho) y$ and expected welfare is given by (3.44):

$$
E\left[W^{N}\right]=-\frac{(1+\rho)^{2}}{\alpha}+\frac{(2+\rho) \rho}{\gamma}
$$

We first find the optimal value of the policy parameter $\rho$ that maximises (3.44). The second derivative test (with respect to $\rho$ ) indicates that the only critical point is, in fact, a maximum. Thus, it is optimal for the CB to commit to:

$$
\rho^{N}=-1
$$

which is effectively a one-to-one rule, in that the CB aims to fully offset the shock to the best of its ability. Expressions (3.36) and (3.47) together imply that the the 
population becomes completely passive:

$$
\kappa_{2 i}^{N}=\kappa_{2}^{N}=0
$$

in that agents find it optimal to set their action equal to zero. Substituting $\rho^{N}=-1$ into expected equilibrium welfare (3.44) yields:

$$
E\left[W^{N}\right]_{\rho=\rho^{N}}=-\frac{1}{\gamma}
$$

where the volatility loss is equal to negative variance of CB's forecast errors about the shock.

Expression (3.49) indicates that, if the CB commits to a one-to-one policy rule, CBT becomes neutral, which is apparent due to the absence of the public signal precision $\alpha$ in the expression. The $\mathrm{CB}$ has to commit to fully offsetting the shock for any transparency setting that is not full CBT. Consider the case where the CB commits to partially offsetting the state variable and makes an announcement with limited transparency $(0<\alpha<\gamma)$. In order to minimise expected loss, agents use the readily observable signal $y$ to align own action with the portion of the shock not offset by the CB. Since the quality of $y$ is lower than the quality of CB's private information, agents are worse off reacting to the public signal, compared to the situation where the CB fully offsets the shock using the more accurate $z$. Thus, a benevolent $\mathrm{CB}$ chooses $\rho=-1$ and individual agents ignore the public signal. If the $\mathrm{CB}$ commits to zero CBT, then agents have no choice but to be passive, as they have no access to any information about $\theta$. Volatility then only depends on CB's ability to match the state variable, which, in turn, depends on the quality of CB's private signal.

When determining the optimal policy setting, we excluded the case of full CBT in the first order condition. If we maximise expected equilibrium welfare in (3.44) with respect to the quality of the public signal $\alpha$, we find that substituting $\alpha=\gamma$ into (3.44) gets rid of the policy parameter $\rho$ and yields the same welfare outcome as commitment to $\rho^{N}=-1$. This leaves the $\mathrm{CB}$ with a choice to either commit to an optimal policy rule and then choose an $\alpha$ from $[0, \gamma]$ (crucially, in that order ${ }^{13}$ ), or to commit to full CBT and no instrument adjustment. Both options yield the same welfare outcome, which is also the welfare outcome in the no-policy case. For the purpose of clarity, in this Chapter we reserve commitment to full CBT for our discussions of no-policy model variations and commitment to $\rho^{N}=-1$ for the

\footnotetext{
${ }^{13}$ The order is only important to distinguish between the cases $(\rho=-1, \alpha=\gamma)$ and $(\alpha=\gamma, \rho=$ $0)$.
} 
analysis of with-policy variations.

\subsection{Fixed Cost: Morris-Shin Payoff Function}

\subsubsection{In the absence of policy}

The first scenario we explore is a fixed information cost setting with M-S payoff. From (3.1), agent $i$ 's payoff is given by:

$$
u_{i}=-\left(a_{i}-\theta\right)^{2}-r\left(L_{i}-\bar{L}\right)-\nu_{i} p
$$

where $\nu_{i} \in\{0,1\}$ and $p$ is the fixed information cost. Agent $i$ is facing a discrete choice of either purchasing a private signal $\left(\nu_{i}=1\right)$ or not purchasing a signal $\left(\nu_{i}=0\right)$.

In order to characterise information acquisition, we seek to determine the values for $p$ that are consistent with the existence of all-purchasing Nash equilibria (P-NE) and non-purchasing Nash equilibria (N-NE). More specifically, we compare agent $i$ 's expected payoffs for (i) when agent $i$ deviates from the rest of the population i.e. $\nu_{i} \neq \nu_{j}$ for $i \neq j$, and (ii) when agent $i$ does not deviate from the rest of the population i.e. $\nu_{i}=\nu_{j}$ for $i \neq j$. By comparing the payoffs we work out a critical price value for which agent $i$ is indifferent between deviating and not deviating. We perform the comparison for when agent $i$ is considering deviating from a purchasing population and then a non-purchasing population. In the former case, we obtain a value for $p_{\max }$ such that $0<p<p_{\max }$ is a sufficient condition for the existence of a $\mathrm{P}-\mathrm{NE}$. In the latter case, we obtain a value for $p_{\min }$ such that $p_{\min }<p$ is a sufficient condition for the existence of a N-NE. In the derivation below we make use of the fact that agent $i$ 's actions in isolation have a negligible effect on the strategies of other agents and the population profile (due to there being an infinite number of agents), thus any such effect is ignored.

\subsubsection{Purchasing equilibria}

Given optimal action characterised by (3.32)-(3.33), agent $i$ 's expected payoff is given by:

$$
E_{i}\left[u_{i}^{P}\right]=-\frac{(1+r)^{2} \alpha+\beta}{[(1+r) \alpha+\beta]^{2}}-p,
$$

which is obtained by substituting (3.3), (3.8), (3.12), and (3.14) into (3.1) and taking expectations. Agent $i$ expects to suffer the above loss in the event where all agents decide to purchase a private signal, including agent $i$. In this case agent $i$ does not 
deviate from the population profile $\left(\nu_{i}=\nu_{j}=1\right.$ for $\left.i \neq j\right)$.

When all agents are purchasing and agent $i$ deviates $\left(\nu_{i}=0\right.$ and $\nu_{j}=1$ for $i \neq j$ ), agent $i$ 's payoff is:

$$
u_{i}^{P d}=-\left(a_{i}^{P d}-\theta\right)^{2}-r\left(L_{i}^{P d}-\bar{L}^{P}\right)
$$

where superscript $P d$ stands for deviating from a purchasing population, $a_{i}^{P d}$ is deviating agent $i$ 's action, $L_{i}^{P d}=\int_{0}^{1}\left(a_{j}^{P}-a_{i}^{P d}\right)^{2} d j$ and $\bar{L}^{P}$ is not affected by agent $i$ 's deviation. We repeat the steps performed in Section 3.2.4.3. Agent $i$ 's optimal action maximises $u_{i}^{P d}$ in (3.51):

$$
a_{i}^{P d}=\frac{1}{1+r} E_{i}[\theta \mid y]+\frac{r}{1+r} E_{i}\left[\bar{a}^{P}\right] .
$$

Agent $i$ 's action can also be represented as:

$$
a_{i}^{P d}=\kappa_{2}^{P d} y
$$

Agent $i$ 's expectation of the average action is:

$$
E_{i}\left[\bar{a}^{P}\right]=\kappa_{1}^{P} E_{i}[\theta \mid y]+\kappa_{2}^{P} y=y
$$

where the weights reflect agent $j$ 's $(j \neq i)$ best response to information from agent $i$ 's perspective. Since the effect of agent $i$ 's actions on the population strategies is negligible, it is safe to assume that agent $j$ behaves in exactly the same way, as in the case where all agents are purchasing. Thus, $\kappa_{1}^{P}$ and $\kappa_{2}^{P}$ are taken directly from (3.32)-(3.33). Comparison of the right-hand side in (3.52) and (3.53) yields:

$$
\kappa_{2}^{P d}=1
$$

Given optimal action, agent $i$ 's expected payoff is given by:

$$
E_{i}\left[u_{i}^{P d}\right]=-\frac{(1+r)^{2} \alpha^{2}+(2+r) \alpha \beta+(1+r) \beta^{2}}{\alpha[(1+r) \alpha+\beta]^{2}} .
$$

Agent $i$ expects to suffer the loss in (3.55) in the event where all agents decide to purchase a private signal, except for agent $i$. In this case agent $i$ deviates from the population profile.

Unsurprisingly, agent $i$ 's expected payoffs in the two cases (not deviating and deviating), as given in (3.50) and (3.55), are not the same. To aid the comparison, 
we split the two expressions into separate terms:

$$
\begin{aligned}
E_{i}\left[u_{i}^{P}\right] & =-\frac{\alpha\left(\kappa_{1}^{P}\right)^{2}+\beta\left(\kappa_{2}^{P}\right)^{2}}{\alpha \beta}-\frac{2 r\left(\kappa_{1}^{P}\right)^{2}}{\beta}+\frac{2 r\left(\kappa_{1}^{P}\right)^{2}}{\beta}-p \\
E_{i}\left[u_{i}^{P d}\right] & =-\frac{1}{\alpha} \quad-\frac{r(\alpha+\beta)\left(\kappa_{1}^{P}\right)^{2}}{\alpha \beta}+\frac{2 r\left(\kappa_{1}^{P}\right)^{2}}{\beta} .
\end{aligned}
$$

The first terms in (3.56) and (3.57) represent the loss from the mismatch between agent $i$ 's action and the state variable $\theta$. The informational value of $x_{i}$ is strictly positive by definition. By forgoing the opportunity to purchase a private signal, agent $i$ reduces own ability to estimate $\theta$ and with that the ability to match it. Thus, the loss in the first term in (3.57) is greater than in (3.56). The next two terms in each equation are the two coordination components $-r L_{i}^{P}$ (or $-r L_{i}^{P d}$ in (3.57)) and $+r \bar{L}^{P}$. The latter is assumed to be the same in both cases (deviation and no deviation) and reflects the dispersion of actions across the population due to the heterogeneity of private signals. Respectively, this term depends on the weight put on private signals and the accuracy of said signals. Terms $-r L_{i}^{P}$ and $-r L_{i}^{P d}$, however, are not the same, as they represent the average distance between agent $i$ 's action and the actions of others. When agent $i$ purchases private information and responds to signals the same way as the rest of the population, the term $-r L_{i}^{P}$ only reflects dispersion due to noise $\varepsilon_{i}$. When agent $i$ does not purchase $x_{i}$ and puts a unit weight on the public signal, agent $i$ suffers a loss from both heterogeneity of private signals and the excess weight on $y$ compared to the rest of the population $\left(\kappa_{2}^{P}<1\right)$. The two coordination terms in (3.56) cancel each other out due to heterogeneity being of zero-sum nature. The sum $-r L_{i}^{P d}+r \bar{L}^{P}$ in (3.57), on the other hand, is non-neutral and its sign depends on the ratio of signal precisions $(\alpha / \beta)$. Although the coordination loss turns into a coordination gain for $\beta<\alpha$, the additional fundamental loss always offsets this positive effect from not purchasing $x_{i}$. Hence, information cost $p$ aside, $E_{i}\left[u_{i}^{P}\right]$ is always greater than $E_{i}\left[u_{i}^{P d}\right]$.

Agent $i$ 's optimal strategy with regard to purchasing $x_{i}$ is as follows:

$$
\begin{cases}\text { if } p<p_{\max }, & \nu_{i}=1 \\ \text { if } p=p_{\max }, & \nu_{i} \in\{0,1\} \\ \text { if } p_{\max }<p, & \nu_{i}=0,\end{cases}
$$

where $p_{\text {max }}$ is the critical value for which $E_{i}\left[u_{i}^{P}\right]=E_{i}\left[u_{i}^{P d}\right]$.

In a symmetric Nash equilibrium none of the individual agents have the incentive to deviate from the actions of others. We can now find the values of price $p$ that are 
consistent with the existence of a symmetric Nash equilibrium in which all agents are purchasing a private signal (P-NE). In order for a symmetric NE to exist, agent $i$ should find it not worthwhile to deviate from the population profile. In other words, the expected payoff in (3.50) should be strictly greater than in (3.55). When the price $p$ is such that the two payoff are equal, it is possible for a $\mathrm{P}-\mathrm{NE}$ to materialise, however, it is not guaranteed, as agent $i$ is indifferent between deviating and not deviating (buying and not buying $x_{i}$ ). Solving the inequality:

$$
E_{i}\left[u_{i}^{P}\right]>E_{i}\left[u_{i}^{P d}\right]
$$

for $p$ yields:

$$
0<p<\frac{(1+r) \beta(\alpha+\beta)}{\alpha[(1+r) \alpha+\beta]^{2}}
$$

where $p_{\max } \equiv \frac{(1+r) \beta(\alpha+\beta)}{\alpha[(1+r) \alpha+\beta]^{2}}$. The above critical value is always positive. First derivatives indicate that $p_{\max }$ is strictly increasing in the quality of private signals $\beta$ and strictly decreasing in the quality of the public announcement $\alpha$. This is an intuitive result, as agent $i$ is willing to purchase information at a higher price if said information is of higher quality. Similarly, a more informative public signal (that is free and readily available) makes private information acquisition less worthwhile for agent $i$, who is, thus, more willing to deviate.

\subsubsection{Welfare and CBT in P-NE}

Turning to CB's policy choice, we substitute purchasing best-response weights from (3.32)-(3.33) into (3.42) to obtain P-NE expected equilibrium welfare:

$$
E\left[W^{P}\right]=-\frac{(1+r)^{2} \alpha+\beta}{[(1+r) \alpha+\beta]^{2}}-p
$$

First derivative of (3.59) with respect to the accuracy of the public signal $\alpha$ yields an ambiguous result. The numerical examples in Figure 3.1 demonstrate the two alternative shapes of expected equilibrium welfare as a function of $\alpha^{14}$. We briefly abstract from the matter of information cost by setting $p=0$ and focusing on the relationship between $E\left[W^{P}\right], \alpha, \beta, \gamma$ and $r$. Expected equilibrium welfare in (3.59) either monotonically increases in $\alpha$ or has an interior minimum in $\alpha$. The interior minimum arises due to the effect discussed by Morris and Shin (2002), who point out that an increase in CBT can be detrimental to welfare if informational value is relatively low and agents pay excessive attention to public announcements. In the

\footnotetext{
${ }^{14}$ In the first example the expected equilibrium welfare expression is identical to that obtained by setting $r=1 / 4$ and $\beta=1$ in the original Morris-Shin model $(r=3 / 4$ and $\beta=1$, in the second example).
} 

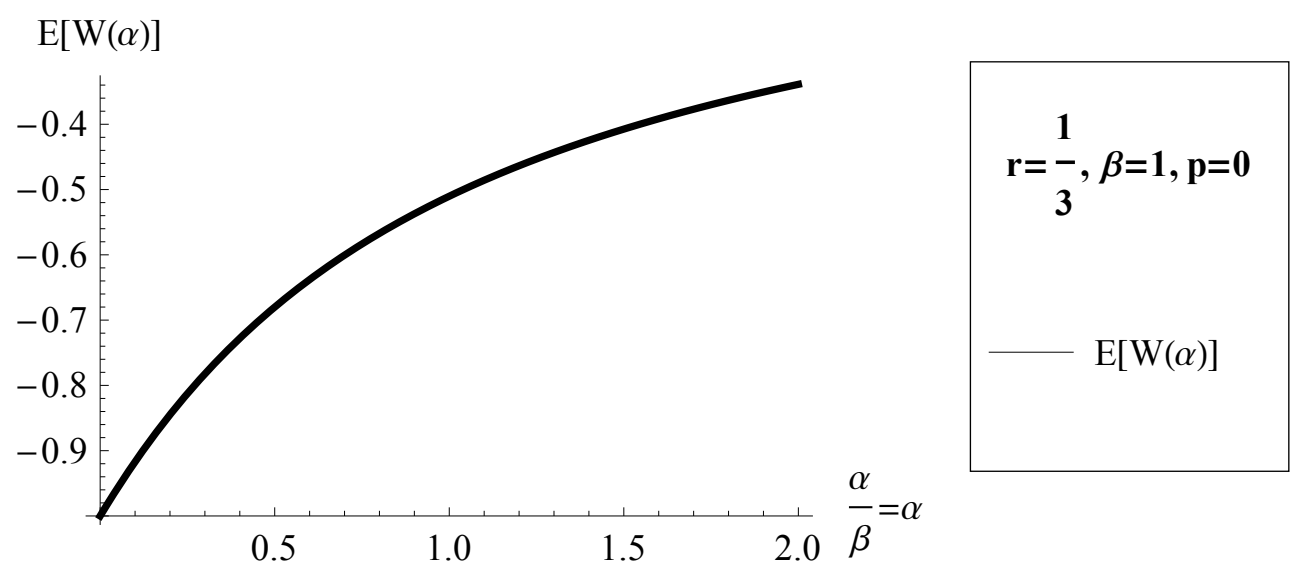

$\mathrm{E}[\mathrm{W}(\alpha)]$
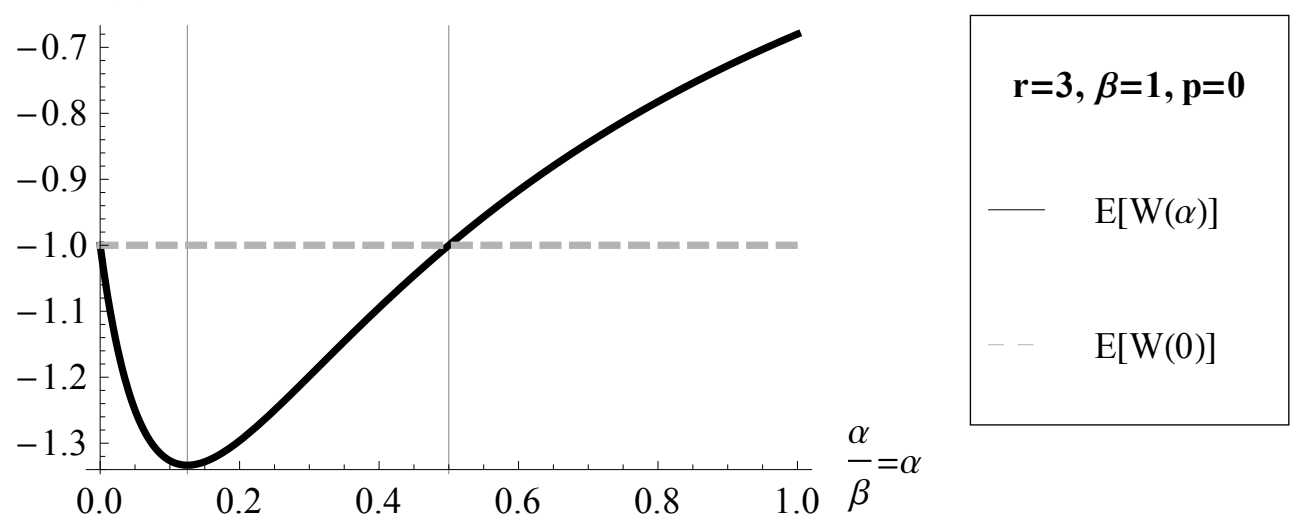

Figure 3.1: Fixed cost. Shapes of expected equilibrium welfare in P-NE (M-S)

former case, the optimal choice for $\alpha$ is its maximum feasible value i.e. the accuracy of the CB's own signal $\gamma$. The latter case involves using $\gamma$ as a limit on $\alpha$ to determine whether zero or full CBT yields the highest expected welfare. Poor quality of CB's private information is associated with $\alpha=0$ being the optimal choice, whereas higher values of $\gamma$ lead to $\alpha=\gamma$ being optimal. Thus, optimal choice of $\alpha$ depends on the values of $\beta, \gamma$ and $r$ :

\begin{tabular}{ll} 
Conditions & CBT \\
\hline $0<r \leq 1$ & Full $(\alpha=\gamma)$ \\
$1<r \wedge 0<\gamma<\gamma_{1}$ & Zero $(\alpha=0)$ \\
$1<r \wedge \gamma=\gamma_{1}$ & Either full or zero $(\alpha \in\{0, \gamma\})$ \\
$1<r \wedge \gamma_{1}<\gamma$ & Full $(\alpha=\gamma)$
\end{tabular}

where $\gamma_{1}=\frac{(r-1) \beta}{1+r}$. For $1<r$, the ratio $\frac{r-1}{1+r}$ is less than unity leading to $\gamma_{1}<\beta$. Thus, the set of conditions $\left(1<r \wedge 0<\gamma<\gamma_{1}\right)$ can be characterised as the case with a high degree of coordination and the quality of CB's private information being 
poor relative to the quality of signals available for purchase to individual agents ${ }^{15}$. The conditions above are directly comparable with those identified in Morris and Shin (2002) and analysed in more detail in Svensson (2006). The conditions are not identical due to the different definitions of $r$ in the original and the re-formulated Morris-Shin payoff functions. However, Svensson's comments and critique remain valid in the current setting.

Agent $i$ 's expected payoff and expected social welfare expressions that correspond to full and zero transparency are as follows:

$$
\begin{aligned}
& E_{i}\left[u_{i}^{P}\right]_{\alpha=\gamma}=E\left[W^{P}\right]_{\alpha=\gamma}=-\frac{(1+r)^{2} \gamma+\beta}{[(1+r) \gamma+\beta]^{2}}-p \\
& E_{i}\left[u_{i}^{P}\right]_{\alpha=0}=E\left[W^{P}\right]_{\alpha=0}=-\frac{1}{\beta}-p .
\end{aligned}
$$

We can now look at how the condition in (3.58) is affected by CBT considerations. Under zero CBT, an agent who deviates and does not purchase $x_{i}$ - when all other agents acquire private information - has no new information to act on and, thus, does not act at all. The expected loss of a deviating agent is not defined, as (in the absence of policy intervention) there is nothing to offset the shock. This can be demonstrated by setting $\alpha=0$ in (3.55). To avoid undefined expected loss, agent $i$ always chooses to purchase $x_{i}$ in the absence of a public signal. In other words, the critical value for $p$ in (3.58) become infinitely large, and a P-NE exists for all values of $p$.

Under full CBT, the existence of a P-NE is consistent with only a subset of values for price $p$. In the absence of the additional noise in the public signal, the condition in (3.58) becomes:

$$
0<p<\frac{(1+r) \beta(\gamma+\beta)}{\gamma[(1+r) \gamma+\beta]^{2}}
$$

Whether or not a P-NE can materialise crucially depends on the relative quality of the private and public information. To facilitate equilibrium analysis at a later stage we distinguish between four cases:

\footnotetext{
${ }^{15}$ The same characterisation can be given to a subset of conditions $\left(1<r \wedge \gamma_{1}<\gamma<\beta\right)$ for which full CBT is optimal. Thus, this characterisation is not unique to the zero CBT case.
} 


\begin{tabular}{llll} 
& Conditions & CBT & P-NE exists \\
\hline Case A & $0<r \leq 1$ & Full & $0<p<p_{\max }^{P}$ \\
Case B & $1<r \wedge 0<\gamma<\gamma_{1}$ & Zero & $0<p$ \\
Case C & $1<r \wedge \gamma=\gamma_{1}$ & Full/zero & (multiplicity) \\
Case D & $1<r \wedge \gamma_{1}<\gamma$ & Full & $0<p<p_{\max }^{P}$
\end{tabular}

where $\gamma_{1}=\frac{(r-1) \beta}{1+r}$, and $p_{\max }^{P} \equiv \frac{(1+r) \beta(\gamma+\beta)}{\gamma[(1+r) \gamma+\beta]^{2}}$ from $(3.62)$.

\subsubsection{Non-purchasing equilibria}

Given that optimal action is equal to $y$, as suggested by (3.37), agent $i$ 's expected payoff is:

$$
E_{i}\left[u_{i}^{N}\right]=-\frac{1}{\alpha}
$$

which is obtained by substituting (3.8), (3.13), and (3.15) into (3.1) and taking expectations. Agent $i$ expects to suffer the loss in (3.63) in the event where no agents purchase private information, including agent $i$. In this case agent $i$ does not deviate from the population profile $\left(\nu_{i}=\nu_{j}=0\right.$ for $\left.i \neq j\right)$.

When no agents are purchasing and agent $i$ deviates $\left(\nu_{i}=1\right.$ and $\nu_{j}=0$ for $i \neq j$ ), agent $i$ 's payoff becomes:

$$
u_{i}^{N d}=-\left(a_{i}^{N d}-\theta\right)^{2}-r\left(L_{i}^{N d}-\bar{L}^{N}\right),
$$

where superscript $N d$ stands for deviating from a non-purchasing population, $a_{i}^{N d}$ is deviating agent $i$ 's action, $L_{i}^{N d}=\int_{0}^{1}\left(a_{j}^{N}-a_{i}^{N d}\right)^{2} d j$ and $\bar{L}^{N}=0$ is unaffected by agent $i$ 's action. Agent $i$ 's optimal action maximises $u_{i}^{N d}$ in (3.64):

$$
a_{i}^{N d}=\frac{1}{1+r} E_{i}\left[\theta \mid x_{i}, y\right]+\frac{r}{1+r} E_{i}\left[\bar{a}^{N}\right]
$$

Agent $i$ 's action can also be represented as:

$$
a_{i}^{N d}=\kappa_{1}^{N d} x_{i}+\kappa_{2}^{N d} y .
$$

Agent $i$ 's expectation of the average action is:

$$
E_{i}\left[\bar{a}^{N}\right]=y .
$$


Comparing (3.65) and (3.66) yields:

$$
\begin{aligned}
\kappa_{1}^{N d} & =\frac{\beta}{(1+r)(\alpha+\beta)} \\
\kappa_{2}^{N d} & =1-\kappa_{1}^{N d} .
\end{aligned}
$$

Given optimal action, agent $i$ 's expected payoff is given by:

$$
E_{i}\left[u_{i}^{N d}\right]=-\frac{\alpha+r(\alpha+\beta)}{(1+r) \alpha(\alpha+\beta)}-p
$$

Agent $i$ expects to suffer the above loss in the event where all agents decide to not purchase a private signal, whereas agent $i$ chooses to do so. In this case agent $i$ deviates from the population profile.

Agent $i$ 's optimal strategy for not acquiring a private signal is:

$$
\begin{cases}\text { if } p<p_{\text {min }}, & \nu_{i}=1 \\ \text { if } p=p_{\text {min }}, & \nu_{i} \in\{0,1\} \\ \text { if } p_{\text {min }}<p, & \nu_{i}=0,\end{cases}
$$

where $p_{\min }$ is the critical value for which $E_{i}\left[u_{i}^{N}\right]=E_{i}\left[u_{i}^{N d}\right]$.

Extrapolating from agent $i$ to the entire population, we can now find the values of price $p$ that are consistent with the existence of a symmetric Nash equilibrium in which information acquisition does not occur (N-NE). The expected payoff in (3.63) should be strictly greater than in (3.68). When the price $p$ is such that the two payoffs are equal, it is possible for a N-NE to materialise, however, it is not guaranteed, as agent $i$ is indifferent between deviating and not deviating (buying and not buying $x_{i}$ ). Solving the inequality:

$$
E_{i}\left[u_{i}^{N}\right]>E_{i}\left[u_{i}^{N d}\right]
$$

for $p$ yields:

$$
\frac{\beta}{(1+r) \alpha(\alpha+\beta)}<p
$$

where $p_{\min } \equiv \frac{\beta}{(1+r) \alpha(\alpha+\beta)}$. The above critical value is always positive, strictly decreasing in $\alpha$, strictly increasing in $\beta$ and strictly decreasing in $r$. Consider a case where the acquisition cost is equal to $p_{\min }$ and agent $i$ is indifferent between purchasing and not purchasing $x_{i}$. A marginal increase in the quality of public information $\alpha$ pushes $p_{\text {min }}$ just below the actual value of price, as a more precise public signal 
makes private information relatively less appealing. An agent that was previously indifferent between the two acquisition choices now strictly prefers to deviate. The exact opposite is true for marginal changes in the quality of private information $\beta$. As far as the coordination parameter is concerned, the more beneficial coordination becomes, as perceived by agent $i$, the more agent $i$ is willing to align own action with the population profile. Thus, a marginal increase in $r$ makes a formerly indifferent agent forego information acquisition with certainty.

In the absence of policy and information acquisition, the $\mathrm{CB}$ commits to full CBT and the expected welfare outcome is given by (3.46), where $E_{i}\left[u_{i}^{N}\right]_{\alpha=\gamma}=E\left[W^{N}\right]_{\alpha=\gamma}$. The critical value of $p$, above which a N-NE exists, under full transparency becomes:

$$
p_{\min }^{N} \equiv \frac{\beta}{(1+r) \gamma(\gamma+\beta)}
$$

\subsubsection{Equilibrium conditions for P-NE and N-NE}

We can now bring together the conditions for existence of purchasing and nonpurchasing equilibria and discuss how acquisition cost determines the equilibrium outcome for different combinations of parameter values. We separately look at the cases identified for the P-NE, which differ in optimal CBT:

\begin{tabular}{llll} 
& Conditions & CBT & P-NE exists \\
\hline Cases A/D & $(0<r \leq 1) \vee\left(1<r \wedge \gamma_{1}<\gamma\right)$ & Full & $0<p<p_{\text {max }}^{P}$ \\
Case B & $1<r \wedge 0<\gamma<\gamma_{1}$ & Zero & $0<p$ \\
Case C & $1<r \wedge \gamma=\gamma_{1}$ & Full/zero & (multiplicity)
\end{tabular}

Although initially we identified four cases, for the purpose of this analysis we look at Cases A and D as a single case, since both cases imply full CBT as the optimal communication policy for P-NE.

A brief visual representation of the equilibrium conditions and optimal CBT is given in Figure 3.2. We start with Cases $\mathbf{A}$ and $\mathbf{D}$ which are characterised by full CBT being optimal for both P-NE and N-NE. The corresponding parameter value combinations are either $0<r \leq 1$ or $1<r \wedge 0<\gamma_{1}<\gamma$, where $\gamma_{1}=\frac{(r-1) \beta}{1+r}$. This implies either a weak coordination motive (Case A) or a strong coordination motive and a lower boundary on the quality of the public information (Case D). The lower boundary $\gamma_{1}$ has the form of a fraction of the quality of purchased private information. The coefficient on $\beta$ lies in the interval $(0,1)$, which means that in Case D the quality of public information can be lower or higher than the quality 
of private signals. In Cases $\mathrm{A}$ and $\mathrm{D}$, the critical price $p_{\max }^{P}$ is strictly greater than $p_{\text {min }}^{N}$ (both critical prices are evaluated at full CBT). The two price intervals that correspond to the existence of either P-NE or N-NE overlap. For $0<p<p_{m i n}^{N}$, only P-NE exist; for $p=p_{\text {min }}^{N}$, a P-NE exists, but a N-NE may exist as well; for $p_{\min }^{N}<p<p_{\max }^{P}$, both P-NE and N-NE exist (multiplicity of equilibria); for $p=p_{\max }^{P}$, a N-NE exists, but a P-NE may exist; and for $p_{\max }^{P}<p$, only N-NE exist. Full CBT is optimal for all values of $p$. From (3.60) and (3.46), the relevant expected equilibrium welfare outcomes are:

$$
\begin{aligned}
& E\left[W^{P}\right]_{\alpha=\gamma}=-\frac{(1+r)^{2} \gamma+\beta}{[(1+r) \gamma+\beta]^{2}}-p \\
& E\left[W^{N}\right]_{\alpha=\gamma}=-\frac{1}{\gamma}
\end{aligned}
$$

the realisation of which depends on the value of $p$.

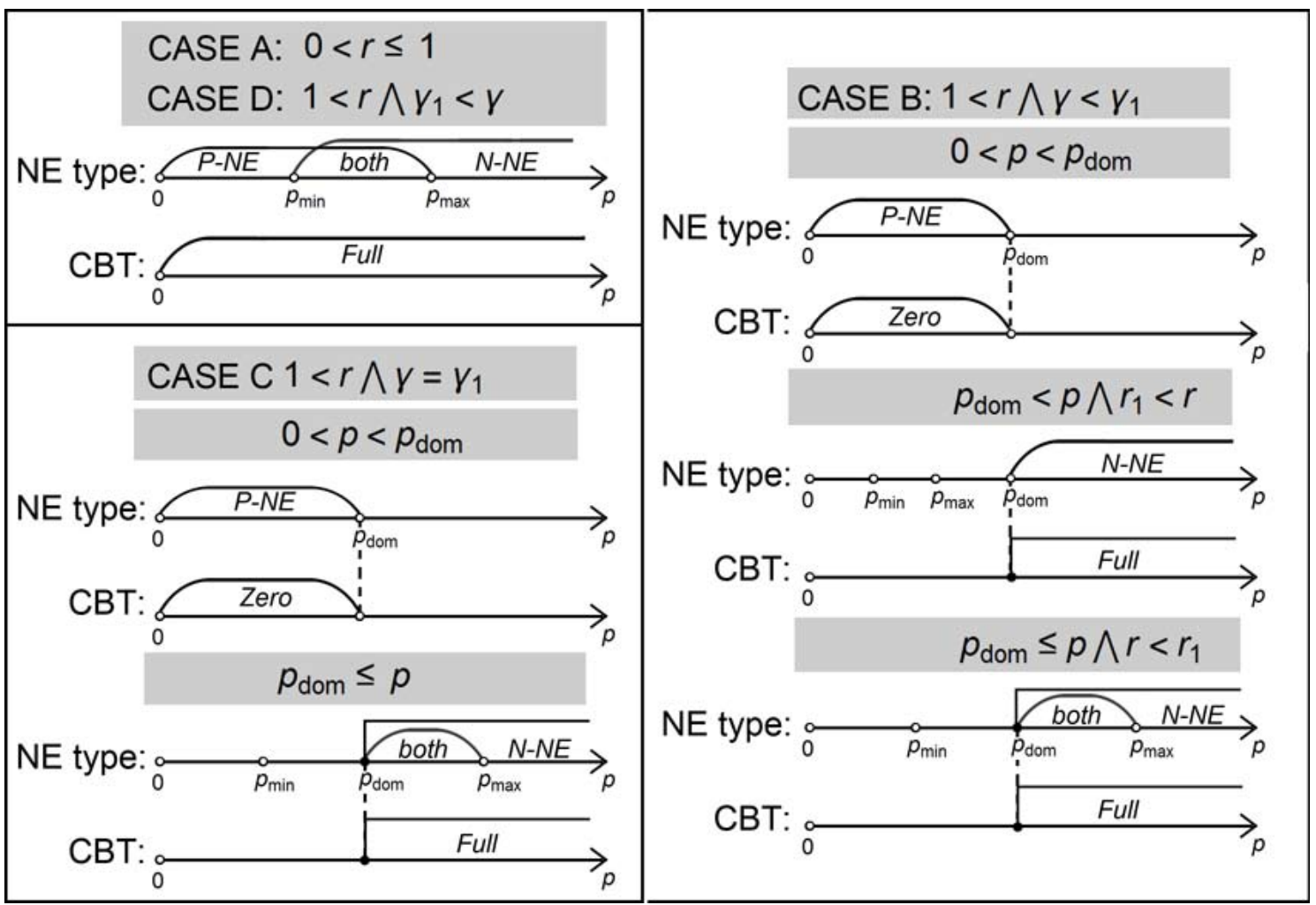

Figure 3.2: Fixed cost. Descriptions of equilibrium cases and CBT decisions (M-S)

We now move on to Case B which corresponds to the combination of a strong coordination motive and poor quality of public information, i.e. $1<r \wedge 0<\gamma<\gamma_{1}$. The critical price $p_{\max }^{P}$ (evaluated for zero CBT) approaches infinity, meaning that P-NE exist for all values of $p$. N-NE exist for $p_{\min }^{N}<p$ (where $p_{\min }^{N}$ is evaluated for full CBT). Thus, there is a multiplicity of equilibria over the interval $p_{\min }^{N}<p$. At $p=p_{m i n}^{N}$, a P-NE exists, but a N-NE can exist as well. Over the price range, 
where the existence of the two NE types overlaps, the CB faces a conflict of optimal setting of CBT, as zero CBT is optimal for P-NE and full CBT is optimal for N-NE. In order to address this conflict the equilibrium solution can be further analysed by asking if the CB views one NE type to be more preferable than the other.

In order to determine if one NE type is socially preferable to the other, we compare the two expressions for expected social welfare, from (3.61) and (3.46):

$$
\begin{aligned}
& E\left[W^{P}\right]_{\alpha=0}=-\frac{1}{\beta}-p \\
& E\left[W^{N}\right]_{\alpha=\gamma}=-\frac{1}{\gamma} .
\end{aligned}
$$

We find that the two are equal for $p=\frac{1}{\gamma}-\frac{1}{\beta}$, where $p_{\text {dom }} \equiv \frac{1}{\gamma}-\frac{1}{\beta}$. Thus, for $0<p<$ $p_{\text {dom }}$, the P-NE welfare-dominate the N-NE, and the opposite is true for $p_{\text {dom }}<p$. Not surprisingly, due to the presence of informational inefficiencies in equilibria neither of the previously derived critical prices that define the existence of P-NE and N-NE $\left(p_{\max }^{P}, p_{\min }^{N}\right)$ coincide with the critical price characterising preference at the aggregate level. The question is whether the CB can use the only instrument at its disposal - CBT - to induce a socially preferable NE. First, we assume that the $\mathrm{CB}$ commits to zero CBT for all prices below the critical value $p_{\text {dom }}$. Both critical values, from (3.58) and (3.69):

$$
\begin{aligned}
& p_{\text {max }}^{P}=\frac{(1+r) \beta(\alpha+\beta)}{\alpha[(1+r) \alpha+\beta]^{2}} \\
& p_{\text {min }}^{N}=\frac{\beta}{(1+r) \alpha(\alpha+\beta)},
\end{aligned}
$$

become infinitely large in the limit as the quality of the public signal approaches zero. In this case a P-NE exists for all prices and no N-NE exist. Thus, the CB is successfully able to induce a socially preferable P-NE by setting $\alpha=0$ for $0<p<p_{\text {dom }}$, in which case the expected welfare outcome is $E\left[W^{P}\right]_{\alpha=0}$.

Secondly, we assume that the CB commits to full CBT over the information price range $p_{\text {dom }}<p$, for which N-NE are socially preferable. We evaluate the critical prices $p_{\max }^{P}$ and $p_{\min }^{N}$ at $\alpha=\gamma$ and compare the results with the value of $p_{\text {dom }}<p$. We find that $\left.p_{\min }\right|_{\alpha=\gamma}$ is strictly smaller than $p_{\text {dom }}<p$, which implies that a N-NE exists over the target price range under full CBT. However, the relationship between $\left.p_{\max }^{P}\right|_{\alpha=\gamma}$ and $p_{\text {dom }}<p$ is ambiguous, and in order to determine the optimal CBT 
setting further conditions on parameter value combinations need to be imposed. If:

$$
\frac{1}{2(\beta-\gamma) \gamma^{2}}\left[\beta^{3}-\beta^{2} \gamma+2 \gamma^{3}+\beta \sqrt{\beta(\beta+\gamma)\left(\beta^{2}-3 \beta \gamma+4 \gamma^{2}\right)}\right]<r
$$

$\left.p_{\max }^{P}\right|_{\alpha=\gamma}$ is strictly smaller than $p_{\text {dom }}<p$, and only N-NE exist over the range $p_{\text {dom }}<p$. In this case full CBT is optimal, only the socially preferable NE type exists, and the only possible expected social welfare outcome is:

$$
E\left[W^{N}\right]_{\alpha=\gamma}=-\frac{1}{\gamma}
$$

If the above condition on $r$ is not met, there is a subset of prices ( $p_{\text {dom }}<p \leq$ $\left.p_{\max }^{P}\right|_{\alpha=\gamma}$ ) for which both NE types exist (or may exist). Since an N-NE is socially preferable for this price range, it is optimal for the $\mathrm{CB}$ to commit to full CBT, otherwise an N-NE would not exist. If, however, a P-NE occurs under full CBT, agent $i$ is expected to suffer a loss that is greater than it would be under zero CBT. Hence, the CB chooses to commit to full CBT when the private information cost is above $p_{\text {dom. }}$. For $p_{\text {dom }}<p \leq\left. p_{\text {max }}^{P}\right|_{\alpha=\gamma}$, the expected social welfare outcome is one of the two:

$$
\begin{aligned}
& E\left[W^{P}\right]_{\alpha=\gamma}=-\frac{(1+r)^{2} \gamma+\beta}{[(1+r) \gamma+\beta]^{2}}-p \\
& E\left[W^{N}\right]_{\alpha=\gamma}=-\frac{1}{\gamma}
\end{aligned}
$$

whereas, for $\left.p_{\max }^{P}\right|_{\alpha=\gamma}<p$, the only possible outcome is:

$$
E\left[W^{N}\right]_{\alpha=\gamma}=-\frac{1}{\gamma}
$$

Finally, if the information cost is exactly equal to $p_{\text {dom }}$, the CB can commit to either full or zero CBT with equal expected social welfare outcome.

In Case C, the expected social welfare in a P-NE is identical under zero and full CBT, thus the CB is indifferent between the two communication policies. The associated critical values of price that binds the interval for which P-NE exist are different under the two CBT regimes. Under full CBT, a P-NE exists with certainty for:

$$
0<p<\frac{2(1+r)}{(r-1) r \beta}
$$

where $\left.p_{\max }^{P}\right|_{\alpha=\gamma=\gamma_{1}}=\frac{2(1+r)}{(r-1) r \beta}$, whereas, under zero CBT, a P-NE exists for all $p$. 
Under full CBT, a N-NE exists with certainty for:

$$
\frac{1+r}{2(r-1) r \beta}<p
$$

where $\left.p_{m i n}^{N}\right|_{\alpha=\gamma=\gamma_{1}}=\frac{1+r}{2(r-1) r \beta}$, and a N-NE does not exist at all under zero CBT. Since $p_{\min }^{N}$ is finite and $p_{\min }^{N}<p_{\max }^{P}$ (when $\alpha=\gamma=\gamma_{1}$ ), there is always a price range over which both types of NE exist.

When the information cost is below the critical value $\left.p_{\max }^{P}\right|_{\alpha=\gamma=\gamma_{1}}$, the $\mathrm{CB}$ can commit to either zero or full CBT with no consequence for social welfare. For the remaining prices, the $\mathrm{CB}$ makes the choice of communication policy the same way as in Case B - based on what NE type is socially preferable for any given value of information cost. As before, comparing the expressions for expected welfare corresponding to the two NE types, from (3.61) and (3.46):

$$
\begin{aligned}
& E\left[W^{P}\right]_{\alpha=0, \gamma=\gamma_{1}}=E\left[W^{P}\right]_{\alpha=\gamma=\gamma_{1}}=-\frac{1}{\beta}-p \\
& E\left[W^{N}\right]_{\alpha=\gamma=\gamma_{1}}=-\frac{1+r}{(r-1) \beta},
\end{aligned}
$$

we find that a P-NE is preferred for $0<p<p_{d o m}$, where $\left.p_{d o m}\right|_{\gamma=\gamma_{1}}=\frac{2}{(r-1) \beta}$. For prices above $p_{d o m}$, a N-NE is socially preferable. Thus, it is optimal for the CB to choose zero CBT for $0<p<p_{\text {dom }}$ to induce P-NE and full CBT for $p_{d o m}<p$ to allow the existence of N-NE. Over the range $p_{\text {dom }}<p<p_{\text {max }}^{P}$ both NE types exist, the existence of P-NE is socially suboptimal, yields a lower expected welfare outcome than a corresponding N-NE, yet cannot be avoided. As in Case B, when $p$ is exactly equal to $p_{\text {dom }}$, the choice of CBT is arbitrary.

\subsubsection{Efficient benchmark}

We now turn to analysing the efficiency of the NE identified and analysed above. In order to do that, we first establish and derive an efficient benchmark case, which is an economy where collectively efficient use and acquisition of information is imposed on private agents by the CB. Since in equilibrium, the CB uses CBT adjustments to influence agents' actions and decisions with the aim of bringing them closer to collectively efficient behaviour, in the efficient case, there is no need for CBT considerations, as all actions are collectively efficient by definition. Thus, the public signal is assumed to have maximum quality $(\alpha=\gamma, y=z)$ from the offset.

Firstly, we establish what is the efficient use of information when all agents are 
purchasing, as characterised by $\tilde{\kappa}_{1}^{P}$ and $\tilde{\kappa}_{2}^{P}$. From (3.38), the welfare is given by:

$$
\tilde{W}^{P}=-\int_{0}^{1}\left(\tilde{a}_{i}^{P}-\theta\right)^{2} d i-p=-\int_{0}^{1}\left(\tilde{\kappa}_{1}^{P} x_{i}+\tilde{\kappa}_{2}^{P} z-\theta\right)^{2} d i-p
$$

We then take expectations of the above expression and maximise it with respect to weights $\tilde{\kappa}_{1}^{P}$ and $\tilde{\kappa}_{2}^{P}$ to find:

$$
\begin{aligned}
& \tilde{\kappa}_{1}^{P}=\frac{\beta}{\beta+\gamma} \\
& \tilde{\kappa}_{2}^{P}=1-\tilde{\kappa}_{1}^{P} .
\end{aligned}
$$

Efficient use of information for a purchasing agent implies a best response that is a weighted average of two signals, where each weight is proportionate to a given signal's quality. Given that agents act efficiently, the expected efficient welfare outcome for a purchasing population is:

$$
E\left[\tilde{W}^{P}\right]=-\frac{1}{\beta+\gamma}-p
$$

Secondly, we repeat the same steps to find the efficient welfare outcome for a non-purchasing economy. From (3.38), the welfare is given by:

$$
\tilde{W}^{N}=-\int_{0}^{1}\left(\tilde{a}_{i}^{N}-\theta\right)^{2} d i=-\int_{0}^{1}\left(\tilde{\kappa}_{2}^{N} z-\theta\right)^{2} d i
$$

the expectation of which is maximised for $\tilde{\kappa}_{2}^{N}=1$. The expected efficient welfare outcome for a non-purchasing population is then:

$$
E\left[\tilde{W}^{N}\right]=-\frac{1}{\gamma}
$$

Thirdly, we derive the condition for efficient information acquisition, which is represented in terms of a critical information cost. The critical price $\tilde{p}$ is defined as the price $p$ that equates the two efficient welfare outcomes $E\left[\tilde{W}^{P}\right]$ and $E\left[\tilde{W}^{N}\right]$. It is collectively efficient for agents to purchase a private signal when:

$$
0<p<\frac{\beta}{\gamma(\beta+\gamma)}
$$

where $\tilde{p} \equiv \frac{\beta}{\gamma(\beta+\gamma)}$. Conversely, it is socially efficient for agents to forgo private information acquisition when $\tilde{p}<p$. Lastly, if actual information cost is equal to the critical value, whether or not the population is a purchasing one has no effect 
on the expected level of social welfare.

\subsubsection{Efficiency of equilibria}

We are now in the position to assess the efficiency of the NE derived for this model variation. The comparison of the efficient benchmark and the different equilibrium cases is presented in full in Appendix C.4. Here we re-iterate some key results from the analysis.

We discuss efficiency with respect to three aspects: (i) information acquisition, (ii) information use, and (iii) expected welfare outcome. As far as information acquisition is concerned, since the efficient critical price $\tilde{p}$ is strictly greater than both $p_{\min }^{N}$ and $p_{\text {dom }}$, there is always a set of prices for which acquisition behaviour is inefficient. By inefficient acquisition we mean that in equilibrium agents find it worthwhile to purchase $x_{i}$, when it is not efficient to do so, and vice versa. Since $\tilde{p}$ and $p_{\text {max }}^{P}$ are positive, there is always a range of $l o w^{16}$ prices, for which purchasing of private information happens in equilibrium and it is, in fact, efficient. Similarly, since $\tilde{p}$ is finite and the price range for which N-NE exist has no upper limit, there is always a range of $h i g h^{17}$ prices, for which no acquisition is happening in equilibrium and such behaviour is efficient.

Information use in $\mathrm{P}-\mathrm{NE}$ is inefficient. Coordination motive distorts the perceived value of private and public information, thereby moving the equilibrium best response to observed information away from the efficient benchmark. The additional loss from this inefficiency means that no P-NE is associated with the first-best welfare outcome. This loss also applies to the expected payoff of an agent who deviates from a non-purchasing population. Conversely, the use of information in N-NE is efficient. The existence of inefficiency in the use of information by a deviating agent versus the efficient use of information by non-deviating agents leads to the following result. N-NE exist for a wider range of $p$ than is efficient. At the same time, $\mathrm{P}-\mathrm{NE}$ exist for a narrower range of $p$ than is efficient, if the quality of CB's private information is sufficiently high ${ }^{18}$. This has to do with the fact that an agent that is deviating from a purchasing information observes a signal of high quality (with no additional noise due to commitment to full CBT) and responds to that signal efficiently, whereas non-deviating agents suffer the aforementioned loss due to inef-

\footnotetext{
${ }^{16}$ Here low stands for a range $\left(0, p^{*}\right)$, where $p^{*}$ is $p_{\min }^{N}$ or $p_{d o m}$, depending on the equilibrium Case.

${ }^{17}$ Here high stands for a range $\left(p^{*},+\infty\right)$, where $p^{*}$ is $\tilde{p}$ or $p_{\max }^{P}$, depending on the equilibrium Case.

${ }^{18}$ Here high refers to precision $\gamma$ being greater than $\frac{\beta \sqrt{1+r}}{1+r}$.
} 
ficient use of information.

In Cases B and C, we speak of the way the CB uses commitment to CBT regimes in order to induce a specific type of NE for a given value of price. More specifically, the CB works out the critical value $p_{\text {dom }}$, below which it is collectively efficient to induce a P-NE. Althought in Cases B and C, as well as the efficient benchmark, the CB is benevolent and aims to maximise expected social welfare, the value for $p_{d o m}$ is strictly lower than $\tilde{p}$. The discrepancy between the two price values is, of course, due to the inefficient use of information in P-NE, which creates an additional social loss and makes N-NE collectively efficient for a wider range of prices.

It should be noted that inefficient use of information is not the only cause of inefficient information acquisition. To demonstrate that, we briefly construct an intermediate setup between the original setup and the efficient benchmark. More specifically, the CB is able to enforce efficient use of information by agents that choose to purchase private signals, but cannot affect the critical prices that define the existence of purchasing and non-purchasing equilibria. Thus, the critical prices $p_{\max }^{P}$ and $p_{\min }^{N}$ are endogenous and characterise the point at which an individual agent is indifferent between deviating or not deviating from a population type (purchasing or non-purchasing). In practical terms, we replace $\kappa_{1}^{P}, \kappa_{2}^{P}, \kappa_{1}^{N d}$, and $\kappa_{2}^{N d}$ in (3.32) and (3.67) with the efficient weights $\tilde{\kappa}_{1}^{P}$ and $\tilde{\kappa}_{2}^{P}$ from (3.75). We then proceed to solve the model and compare the resulting critical prices with $\tilde{p}$. The relationship between $p_{\text {max }}^{P}$ and $\tilde{p}$ is ambiguous ${ }^{19}$, but $p_{\min }^{N}$ is strictly lower than the efficient critical price. This is sufficient to conclude that efficient use of information on its own does not ensure efficient acquisition of information for all values of price. There always remains a range of prices for which information acquisition is inconsistent with the efficient benchmark. This result stems from the presence of a coordination motive at the level of individual agents, which is neutral at the aggregate level. When information acquisition is endogenous, agents take into account the loss from imperfect coordination in determining their strategy for each value of $p$. Whereas, the efficient benchmark for information acquisition is worked out at the level of the economy as a whole, at which the degree of coordination is zero ${ }^{20}$.

Finally, we highlight the fact that first-best expected welfare outcomes are achieved in certain equilibria. This is the case when the existence of N-NE is in line with efficient acquisition of information, i.e. when information cost is sufficiently high ${ }^{21}$.

\footnotetext{
${ }^{19}$ More specifically, $p_{\max }^{P}<\tilde{p}$ for $\beta<\gamma$ and the reverse is true for $\gamma<\beta$.

${ }^{20}$ Coordination neutrality at the aggregate level and not at the level of an individual agent is a key characteristic of the M-S payoff function.

${ }^{21}$ See footnote 17
} 
Efficient use of information by a non-purchasing population ensures the attainment of the first-best welfare outcome.

\subsubsection{With direct policy intervention}

We now introduce direct policy intervention into agents' payoffs, from (3.10):

$$
u_{i}=-\left(a_{i}-\theta-g\right)^{2}-r\left(L_{i}-\bar{L}\right)-\nu_{i} p
$$

where $\nu_{i} \in\{0,1\}$ and $g=\rho z$.

\subsubsection{Purchasing equilibria}

Given optimal action in (3.26)-(3.27), we can find agent $i$ 's expected payoff, the expression for which is omitted here due to its length. In this case agent $i$ does not deviate from the population profile $\left(\nu_{i}=\nu_{j}=1\right.$ for $\left.i \neq j\right)$.

When all agents are purchasing and agent $i$ deviates $\left(\nu_{i}=0\right.$ and $\nu_{j}=1$ for $i \neq j$ ), agent $i$ 's payoff is:

$$
u_{i}^{P d}=-\left(a_{i}^{P d}-\theta-g\right)^{2}-r\left(L_{i}^{P d}-\bar{L}^{P}\right) .
$$

Agent $i$ 's optimal action maximises $u_{i}^{P d}$ in (3.79):

$$
a_{i}^{P d}=\frac{1}{1+r}\left(E_{i}[\theta \mid y]+\rho E_{i}[\theta \mid z]\right)+\frac{r}{1+r} E_{i}\left[\bar{a}^{P}\right],
$$

and can also be represented as:

$$
a_{i}^{P d}=\kappa_{2}^{P d} y
$$

Agent $i$ 's expectation of the average action is:

$$
E_{i}\left[\bar{a}^{P}\right]=\kappa_{1}^{P} E_{i}[\theta \mid y]+\kappa_{2}^{P} y=(1+\rho) y
$$

where the weights reflect agent $j$ 's $(j \neq i)$ best response to information from agent $i$ 's perspective. Comparison of the right-hand side in (3.80) and (3.81) yields:

$$
\kappa_{2}^{P d}=1+\rho
$$

Given optimal action, we find agent $i$ 's expected payoff, which is omitted here due to its length. At this stage it should be noted that agent $i$ 's expected payoffs in the two cases - deviating and not deviating - are not the same. 
Agent $i$ 's optimal strategy for purchasing private information is:

$$
\begin{cases}\text { if } p<p_{\max }, & \nu_{i}=1 \\ \text { if } p=p_{\max }, & \nu_{i} \in\{0,1\} \\ \text { if } p_{\max }<p, & \nu_{i}=0,\end{cases}
$$

where $p_{\max }$ is the critical value for which $E_{i}\left[u_{i}^{P}\right]=E_{i}\left[u_{i}^{P d}\right]$.

We can now find the values of price $p$ that are consistent with the existence of a symmetric Nash equilibrium in which all agents are purchasing a private signal $(\mathrm{P}-\mathrm{NE})$ :

$$
0<p<\frac{(1+r) \beta(\alpha+\beta)[(1+\rho) \gamma-\alpha \rho]^{2}}{\alpha[(1+r) \alpha+\beta]^{2} \gamma^{2}}
$$

where $p_{\max } \equiv \frac{(1+r) \beta(\alpha+\beta)[(1+\rho) \gamma-\alpha \rho]^{2}}{\alpha[(1+r) \alpha+\beta]^{2} \gamma^{2}}$. The critical value $p_{\max }$ is always positive.

\subsubsection{Welfare, instrument adjustment, and CBT in P-NE}

Substituting P-NE best-response weights from (3.26)-(3.27) into (3.40) yield an expression for P-NE expected equilibrium welfare, which is omitted here due to its length. We first maximise expected equilibrium welfare with respect to the policy parameter and then determine the optimal CBT regime.

The sign of $\partial E\left[W^{P}\right] / \partial \rho$ is ambiguous. Having found the critical point, we perform the second derivative test that shows that the identified critical point is a maximum. Thus, the optimal setting for the policy rule $\rho z$ involves:

$$
\rho^{P}=-\frac{\left[(1+r)^{2} \alpha+\beta\right] \gamma}{\beta(\beta+\gamma)+\alpha\left[(1+2 r) \beta+(1+r)^{2} \gamma\right]} .
$$

Given optimal policy, expected equilibrium welfare becomes:

$$
E\left[W^{P}\right]_{\rho=\rho^{P}}=\frac{\rho^{P}}{\gamma}-p
$$

Now we can maximise (3.86) with respect to $\alpha$ to find optimal communication policy. The sign of the first derivative indicates that $E_{i}\left[W^{P}\right]_{\rho=\rho^{P}}$ is monotonically decreasing in $\alpha$. This means that minimum level of CBT is optimal:

$$
E\left[W^{P}\right]_{\rho=\rho^{P}, \alpha=0}=-\frac{1}{\beta+\gamma}-p,
$$


where $\left.\rho^{P}\right|_{\alpha=0}=-\frac{\gamma}{\beta+\gamma}$.

With optimal direct policy intervention and zero CBT, the shock $\theta$ is offset by both agents' actions and instrument adjustment. From (3.38):

$$
W^{P}=-\int_{0}^{1}\left(\kappa_{1}^{P} x_{i}+\kappa_{2}^{P} y-\theta-\rho^{P} z\right)^{2} d i-p,
$$

where the equilibrium weights $\kappa_{1}^{P}$ and $\kappa_{2}^{P}$, when evaluated for $\rho=\rho^{P}$ and zero CBT, become $\frac{\beta}{\beta+\gamma}$ and zero ${ }^{22}$, respectively. As a result, the equilibrium welfare before expectations are taken is equal to:

$$
W^{P}=-\int_{0}^{1}\left(\frac{\beta}{\beta+\gamma} x_{i}-\theta+\frac{\gamma}{\beta+\gamma} z\right)^{2} d i-p .
$$

Agent $i$ knows that the CB is committed to offset a fraction of the shock to the best of its ability, given CB's private signal. Hence, agent $i$ finds it optimal to only match the remainder of the shock, subject to the quality of private information acquired. Thus, the weights on the agents' and CB's actions are proportionate to the quality of information available to the agents and $\mathrm{CB}$, respectively. This makes $\kappa_{1}^{P}$ and $\rho$ the Bayesian weights that would be placed on signals $x_{i}$ and $z$ by a forecaster who could observe both. This result is consistent with that explored by James and Lawler (2011). Under zero transparency, agent $i$ has no incentive to deviate from a purchasing population. This is ensured through optimal setting of policy by the $\mathrm{CB}$, which in this case involves the policy parameter $\rho^{P}$ being strictly below minus unity. A one-to-one rule $(\rho=-1)$ amounts to setting the instrument $g$ equal to minus one times the policymaker's expectation of the shock, which dissuades agents from acting on their private signals. By committing to a value of $\rho$ that lies in the interval $(-1,0)$ the policymaker is not fully offsetting the shock, maintaining the incentive for private information acquisition. In the absence of private and public information, agent $i$ 's expected loss becomes undefined ${ }^{23}$. In order to avoid such an outcome, agents require access to private signals to be able to offset the remainder of the shock. The critical price $p_{\max }$ evaluated at $\rho=\rho^{P}$, in the limit as $\alpha$ approaches zero, is infinitely large. Thus, the condition in (3.84) extends to all positive values, and a P-NE exists for all $0<p$.

\footnotetext{
${ }^{22}$ Agent $i$ ignores the uninformative signal $y$.

${ }^{23}$ This is due to $\theta$ being drawn from an improper uniform prior on the real line, which, in turn, implies that the variance of $\theta$ is not defined.
} 


\subsubsection{Non-purchasing equilibria}

Given that optimal action is $a_{i}^{N}=(1+\rho) y$, as identified in (3.36), agent $i$ 's expected payoff is:

$$
E_{i}\left[u_{i}^{N}\right]=-\frac{\gamma(1+\rho)^{2}-\alpha \rho(2+\rho)}{\alpha \gamma} .
$$

Agent $i$ expects to suffer the loss in (3.88) in the event where no agents purchase private information, including agent $i\left(\nu_{i}=\nu_{j}=0\right.$ for $\left.i \neq j\right)$.

When no agents are purchasing and agent $i$ deviates $\left(\nu_{i}=1\right.$ and $\nu_{j}=0$ for $i \neq j$ ), agent $i$ 's payoff becomes:

$$
u_{i}^{N d}=-\left(a_{i}^{N d}-\theta-g\right)^{2}-r\left(L_{i}^{N d}-\bar{L}^{N}\right)-p .
$$

Agent $i$ 's optimal action maximises $u_{i}^{N}$ in (3.89):

$$
a_{i}^{N d}=\frac{1}{1+r}\left(E_{i}\left[\theta \mid x_{i}, y\right]+\rho E_{i}\left[z \mid x_{i}, y\right]\right)+\frac{r}{1+r} E_{i}\left[\bar{a}^{N}\right]
$$

and can also be represented as:

$$
a_{i}^{N d}=\kappa_{1}^{N d} x_{i}+\kappa_{2}^{N d} y
$$

Agent $i$ 's expectation of the average action is:

$$
E_{i}\left[\bar{a}^{N}\right]=(1+\rho) y
$$

Comparing (3.90) and (3.91) yields:

$$
\begin{aligned}
\kappa_{1}^{N d} & =\frac{\beta[(1+\rho) \gamma-\alpha \rho]}{(1+r)(\alpha+\beta) \gamma} \\
\kappa_{2}^{N d} & =1+\rho-\kappa_{1}^{N d} .
\end{aligned}
$$

Given optimal action, we can find agent $i$ 's expected payoff $E_{i}\left[u_{i}^{N d}\right]$, the expression for which is too long to include here in its entirety, thus, we limit its length by not substituting $\kappa_{1}^{N d}$ :

$$
E_{i}\left[u_{i}^{N d}\right]=-\frac{r\left(\kappa_{1}^{N d}\right)^{2}+\left(\kappa_{1}^{N d}-1-\rho\right)^{2}}{\alpha}-\frac{(1+r)\left(\kappa_{1}^{N d}\right)^{2}}{\beta}-\frac{\left(2 \kappa_{1}^{N d}-2-\rho\right) \rho}{\gamma}-p .
$$


Agent $i$ 's optimal strategy in regard to not purchasing a private signal is:

$$
\begin{cases}\text { if } p<p_{\text {min }}, & \nu_{i}=1 \\ \text { if } p=p_{\text {min }}, & \nu_{i} \in\{0,1\} \\ \text { if } p_{\text {min }}<p, & \nu_{i}=0,\end{cases}
$$

where $p_{\min }$ is the critical value for which $E_{i}\left[u_{i}^{N}\right]=E_{i}\left[u_{i}^{N d}\right]$.

Extrapolating from agent $i$ to the entire population, we can now find the values of price $p$ that are consistent with the existence of a symmetric Nash equilibrium in which information acquisition does not occur (N-NE):

$$
\frac{\beta[(1+\rho) \gamma-\alpha \rho]^{2}}{(1+r) \alpha(\alpha+\beta) \gamma^{2}}<p
$$

where $p_{\min } \equiv \frac{\beta[(1+\rho) \gamma-\alpha \rho]^{2}}{(1+r) \alpha(\alpha+\beta) \gamma^{2}}$. The above critical value is always positive.

Next, we look at the effect optimal choice of policy has on the condition that is necessary for the existence of an N-NE. In a N-NE with policy intervention, the $\mathrm{CB}$ commits to a one-to-one rule and non-deviating agents are passive. Agent $i$ 's expected payoff and expected equilibrium welfare are given by (3.49). If the CB chooses to commit to $\rho^{N}=-1$, then $p_{\min }$ becomes:

$$
\left.p_{\min }\right|_{\rho=\rho^{N}}=\frac{\alpha \beta}{(1+r)(\alpha+\beta) \gamma^{2}},
$$

which is monotonically increasing in $\alpha$ and $\beta$, and monotonically decreasing in $\gamma$ and $r$. Although CBT is neutral at the aggregate level, the quality of public information is still a decision variable at the level of an individual agent that is deciding whether or not to deviate from a non-purchasing population. Thus, the choice of $\alpha$ still affects the value of $p_{\text {min }}$. Given optimal policy, a deviating agent $i$ 's optimal action is characterised by:

$$
\begin{aligned}
\left.\kappa_{1}^{N d}\right|_{\rho=\rho^{N}} & =\frac{\alpha \beta}{(1+r)(\alpha+\beta) \gamma} \\
\left.\kappa_{2}^{N d}\right|_{\rho=\rho^{N}} & =-\kappa_{1}^{N d} .
\end{aligned}
$$

The weight on the public signal is equal to the negative of the weight on the private signal.

We now take a look at what happens to agent $i$ 's payoff in (3.89), once optimal 
policy is taken into account. Firstly, it is worth noting that all private agents that are not deviators (unlike agent $i$ ) are passive (their chosen action is zero), thus, the $-r\left(\bar{L}^{N}\right)$ component of the coordination term is also equal to zero, as there is no variance in actions across the population. The remaining terms in agent $i$ 's payoff are as follows:

$$
\left.u_{i}^{N d}\right|_{\rho=\rho^{N}}=-\left[\kappa x_{i}+\kappa(z-y)+(1-\kappa) z-\theta\right]^{2}-r \kappa^{2}\left(x_{i}-y\right)^{2}-p
$$

where for simplicity of presentation $\left.\kappa \equiv \kappa_{1}^{N d}\right|_{\rho=\rho^{N}}$. Consider, again, that the actual cost of information acquisition $p$ is equal to $\left.p_{\min }\right|_{\rho=\rho^{N}}$ and agent $i$ is indifferent between purchasing and not purchasing $x_{i}$. A marginal increase in the quality of public information $\alpha$ has a positive and a negative effect on a deviating agent's expected payoff:

$$
E_{i}\left[u_{i}^{N d}\right]_{\rho=\rho^{N}}=\left[\frac{(1+2 r) \alpha \beta}{(1+r)^{2}(\alpha+\beta) \gamma^{2}}-\frac{1}{\gamma}\right]-r \cdot \frac{\alpha \beta}{(1+r)^{2}(\alpha+\beta) \gamma^{2}}-p .
$$

On the one hand, a more accurate $y$ means that agent $i$ has a better idea of the true value of the CB's private signal $z$. Agent $i$ is then able to align own action more closely with the CB's forecast error $\left(-\frac{1}{\gamma}\right)$, thereby reducing the combined expected loss from the first two terms in the deviator's expected payoff. On the other hand, an increase in $\alpha$ increases the weight $\kappa$, as the deviator reacts more strongly (more confidently) to available information. The additional confidence stems from the aforementioned increased ability to forecast $z$. This, in turn, forces agent $i$ 's action further away from a passive population profile and increases the coordination loss (third term in the above expression). The positive effect of a marginal increase in $\alpha$ outweighs the additional loss and results in a higher expected payoff overall. A formerly indifferent agent now finds deviation strictly more attractive, thereby raising $p_{\min }$.

Similar reasoning applies to the effect of marginally increasing the quality of private information $\beta$ when agent $i$ is initially indifferent. The positive effect on the expected payoff from being able to match the state variable (net of policy intervention) with more precision outweighs the negative effect from marginally poorer coordination. Thus, agent $i$ strictly prefers deviating from the non-purchasing population and $p_{\min }$ becomes greater. A marginally higher quality of CB's private signal $\gamma$ means that the $\mathrm{CB}$ is better able to offset the shock, increasing the expected payoffs of passive non-deviating agents. This makes agent $i$ less willing to deviate, which expands the set of prices for which a N-NE exists. Lastly, higher $r$ translates into a greater perceived benefit from coordination making non-deviation more 
attractive to agent $i$ and reducing the value of $p_{\min }$.

\subsubsection{Equilibrium conditions for P-NE and N-NE}

A P-NE exists for all values of information cost, whereas a N-NE only exists for $p$ above the critical value $\left.p_{\min }\right|_{\rho=\rho^{N}}$. The latter critical value can take on any value in the range $\left[0, \frac{\beta}{(1+r) \gamma(\beta+\gamma)}\right]$, depending on the choice of the CBT parameter $\alpha$.

\begin{tabular}{llll} 
Type & Condition & Rule & CBT \\
\hline P-NE & $0<p$ & $\left.\rho^{P}\right|_{\alpha=0}=-\frac{\gamma}{\beta+\gamma}$ & $\alpha=0$ (Zero) \\
N-NE & $\left.p_{\min }\right|_{\rho=\rho^{N}}<p$ & $\rho^{N}=-1$ & $0 \leq \alpha \leq \gamma$ (Any)
\end{tabular}

The P-NE and N-NE above are characterised by conflicting optimal setting of the policy parameter $\rho$ and the quality of public information $\alpha$. Should the CB commit to the rule $g=\left.\rho^{P}\right|_{\alpha=0} \cdot z$ for prices above $\left.p_{\min }\right|_{\rho=\rho^{N}}$, the policy setting would be suboptimal for an economy characterised by a non-purchasing NE. Similarly, commitment to $g=-z$ would have a negative effect on the welfare outcome in a P-NE. This creates a problem for the $\mathrm{CB}$ over the range of prices for which both a P-NE and a N-NE exist.

In addressing this conflict of policy, we first ask the question of whether the CB views one type of $\mathrm{NE}$ more preferable than the other over the range of prices for which both NE exist $\left(\left.p_{\min }\right|_{\rho=\rho^{N}}<p\right)$. To do this we investigate whether an economy with a P-NE, zero CBT, and $\rho=\left.\rho^{P}\right|_{\alpha=0}$ outperforms an economy with a N-NE and $\rho=\rho^{N}$ for any values of $p$. We, thus, compare the welfare outcomes from (3.87) and (3.49), and find that $E\left[W^{P}\right]_{\rho=\rho^{P}}$ is greater than $E\left[W^{N}\right]_{\rho=\rho^{N}}$ when:

$$
p<\frac{\beta}{\gamma(\beta+\gamma)}
$$

where $p_{\text {dom }} \equiv \frac{\beta}{\gamma(\beta+\gamma)}$. The above condition represents a price range for which the social loss from the cost of private information acquisition compares favourably to the information loss when the only information about the shock available in the economy is the policymaker's own signal. Over this interval of information cost the P-NE welfare-dominates the N-NE, so it is reasonable to assume that the CB would use its policy instrument and CBT to influence the actions of private agents in such a way so as to induce the socially preferable NE. 


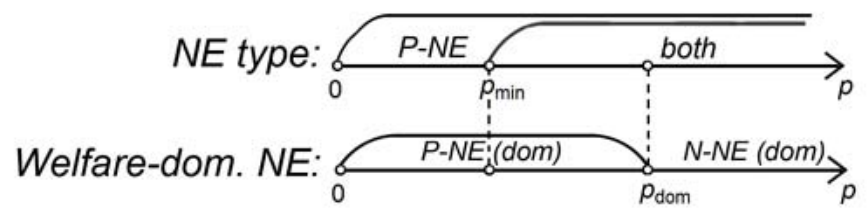

Figure 3.3: Fixed cost. Descriptions of equilibria (M-S with policy)

At this final stage of equilibrium analysis, the $\mathrm{CB}$ aims to use direct policy intervention and CBT to influence the conditions for existence of either NE. The question is whether the $\mathrm{CB}$ is able to encourage or discourage agent $i$ 's decision to deviate from a given type of population. This decision is represented by the expressions for $p_{\max }$ and $p_{\min }$, given by (3.84) and (3.95), respectively:

$$
\begin{aligned}
& 0<p<\frac{(1+r) \beta(\alpha+\beta)[(1+\rho) \gamma-\alpha \rho]^{2}}{\alpha[(1+r) \alpha+\beta]^{2} \gamma^{2}} \\
& \frac{\beta[(1+\rho) \gamma-\alpha \rho]^{2}}{(1+r) \alpha(\alpha+\beta) \gamma^{2}}<p .
\end{aligned}
$$

First, we assume that, when the CB is making its policy decision, it observes the private information cost $p$ to be below the $p_{\text {dom }}$ value. This implies that a P-NE is socially preferable. In this case the $\mathrm{CB}$ wants to drive the value of $p_{\min }$ above the actual value of the price $p$ to ensure that an N-NE no longer exists at $p$, yet a P-NE still exists. We check to see if commitment to a policy rule and CBT that are optimal for a P-NE would be sufficient to achieve this goal. We already know that under commitment to $\rho=\left.\rho^{P}\right|_{\alpha=0}$ in conjunction with zero CBT a P-NE exists for all values of $p$ (as $p_{\max }$ approaches infinity). We now evaluate $p_{\min }$ at $\rho=\left.\rho^{P}\right|_{\alpha=0}$ and $\alpha=0$ and find that $p_{\text {min }}$ also approaches infinity, which means that an NNE does not exist at all for $0<p$. Thus, the CB is successfully able to induce a P-NE for $0<p<p_{\text {dom }}$ while maintaining the optimality of the policy rule and CBT.

Secondly, we assume that the CB observes the actual $p$ to be above the critical value $p_{\text {dom. }}$. Now the reverse is true - a N-NE is socially preferable, hence, the CB aims to decrease the values of $p_{\max }$ and $p_{\min }$ below the actual $p$ so that a P-NE no longer exists for $p$, yet a N-NE exists with certainty. If no agents are acquiring private information, it is optimal for the $\mathrm{CB}$ to offset the shock fully and the choice of CBT has no impact on the level of expected social welfare. We evaluate the 
critical values of $p$ for the two types of equilibrium at $\rho=\rho_{N}$ :

$$
\begin{aligned}
& \left.p_{\text {max }}\right|_{\rho=\rho^{N}}=\frac{(1+r) \alpha \beta(\alpha+\beta)}{[(1+r) \alpha+\beta]^{2} \gamma^{2}} \\
& \left.p_{\text {min }}\right|_{\rho=\rho^{N}}=\frac{\alpha \beta}{(1+r)(\alpha+\beta) \gamma^{2}},
\end{aligned}
$$

and find that both critical values turn to zero if $\alpha$ is set to zero. With $\left.p_{\max }\right|_{\rho=\rho^{N}, \alpha=0}=$ $\left.p_{\min }\right|_{\rho=\rho^{N}, \alpha=0}=0$, a P-NE does not exist and a N-NE exists for all $p$. Thus, by committing to a one-to-one policy rule and zero CBT the CB can successfully induce a N-NE when it is socially preferable to do so.

\begin{tabular}{llll} 
Type & Condition & Rule & CBT \\
\hline P-NE & $0<p \leq p_{\text {dom }}$ & $\left.\rho^{P}\right|_{\alpha=0}=-\frac{\gamma}{\beta+\gamma}$ & $\alpha=0$ (Zero) \\
N-NE & $p_{\text {dom }} \leq p$ & $\rho^{N}=-1$ & $\alpha=0$ (Zero)
\end{tabular}

Finally, it should be noted, that, when $p=p_{\text {dom, }}$, the CB can choose either of the two options: combine zero $\mathrm{CBT}$ with either $\rho=\rho^{P}$ or $\rho=\rho^{N}$. In both cases the expected equilibrium social welfare is the same, as the benefit of private information acquisition is exactly offset by its cost.

\subsubsection{Efficiency of equilibria}

In order to assess the efficiency of equilibria, we use the efficient benchmark previously derived for the setting without policy. The relevant expressions are presented in Table 3.1.

\begin{tabular}{ll|l} 
Equilibrium & Efficient Benchmark \\
\hline$E\left[W^{P}\right]_{\rho=\rho^{P}, \alpha=0}=-\frac{1}{\beta+\gamma}-p$ & $(3.87)$ & $E\left[\tilde{W}^{P}\right]=-\frac{1}{\beta+\gamma}-p$ \\
$E\left[W^{N}\right]_{\rho=\rho^{N}}=-\frac{1}{\gamma}$ & $(3.49)$ & $E\left[\tilde{W}^{N}\right]=-\frac{1}{\gamma}$ \\
$p_{\text {dom }}=\frac{\beta}{\gamma(\beta+\gamma)}$ & $(3.99)$ & $\tilde{p}=\frac{\beta}{\gamma(\beta+\gamma)}$ \\
$\kappa_{1}^{P}=\frac{\beta}{\beta+\gamma}$ & & $\tilde{\kappa}_{1}^{P}=\frac{\beta}{\beta+\gamma}$ \\
$\kappa_{2}^{P}=0,\left.\rho^{P}\right|_{\alpha=0}=-\frac{\gamma}{\beta+\gamma}$ & & $\tilde{\kappa}_{2}^{P}=\frac{\gamma}{\beta+\gamma}$ \\
$\kappa_{2}^{N}=0, \rho^{N}=-1$ & $\tilde{\kappa}_{2}^{N}=1$
\end{tabular}

Table 3.1: Fixed cost. Equilibrium characterisation versus efficient benchmark (M-S with policy) 
Expected equilibrium welfare outcomes in both P-NE and N-NE are, in fact, firstbest. The combination of optimal direct policy intervention and CBT enables the CB to achieve efficient use of information in the economy as well as efficient acquisition of private information by individual agents. The $\mathrm{CB}$ commits to zero CBT for all values of $p$, thereby completely discouraging agents from acting upon public information. To compensate, however, the CB uses its own private information to offset the shock to the best of its ability, ensuring that no informational value is lost. This result is in line with that described in James and Lawler (2011).

\subsubsection{Effect of policy on information acquisition}

As already explained above, the formulation of the M-S payoff function includes a coordination motive that disappears at the aggregate level. As a result, agents perceive coordination as beneficial and, in the absence of direct policy intervention, place excessive weight on public information in P-NE. The combination of inefficient use of information (excess weight on the public signal and underweight on private signals) as well as the presence of a coordination motive only at the individual level leads to the existence of a price range for which private information acquisition is inefficient ${ }^{24}$. Outside of this range, information acquisition is strictly in line with the benchmark case, be it P-NE or N-NE. However, only N-NE correspond to the attainment of the first-best welfare outcome and efficient information use.

On its own optimal setting of CBT is not enough to eliminate equilibrium inefficiencies. However, when optimal CBT is combined with optimally derived instrument adjustment, the $\mathrm{CB}$ is able to induce efficient equilibrium behaviour for all values of $p$. By committing to zero CBT and only partially offsetting the shock over the price range $0<p \leq \tilde{p}$, the $\mathrm{CB}$ induces a $\mathrm{P}-\mathrm{NE}$ when purchasing private signals is, in fact, collectively efficient. Conversely, by committing to a one-to-one policy rule for any price equal to or above $\tilde{p}$, the CB induces a N-NE thereby aligning agents' purchasing behaviour with the efficient benchmark. It should also be noted that direct policy intervention facilitates efficient use of information as well as the attainment of first-best welfare outcomes in both P-NE and N-NE. This result constitutes Proposition 3.1.

Proposition 3.1: If agents aim to maximise a $M$-S payoff function and information

\footnotetext{
${ }^{24}$ For some combinations of values of $\beta, \gamma$ and $r$, a price range exists for which information acquisition is inefficient with certainty. By that we mean that, for instance, only a P-NE exists when no acquisition is collectively efficient, or vice versa. For some combinations of values of $\beta, \gamma$ and $r$, a price range exists for which both types of NE exist, yet only one corresponds to collectively efficient information acquisition. At least one of the two types of price ranges exists for all combination of values of $\beta, \gamma$ and $r$. For specific conditions, see Appendix C.4.
} 
cost $p$ is fixed, commitment to an optimally designed monetary policy rule in combination with optimal level of transparency with respect to the central bank's private signal achieve efficient use of information, efficient information acquisition, as well as the first-best welfare outcome for all values of $p$.

\subsection{Fixed Cost: James-Lawler Payoff Function}

\subsubsection{In the absence of policy}

We repeat the procedure from Section 3.3 with fixed information cost and J-L payoff. From (3.2), agent $i$ 's payoff is given by:

$$
u_{i}=-\left[a_{i}-\theta+r\left(a_{i}-\bar{a}\right)\right]^{2}-\nu_{i} p,
$$

where $\nu_{i} \in\{0,1\}$.

\subsubsection{Purchasing equilibria}

When all agents are purchasing private information $\left(\nu_{i}=\nu_{j}=1\right.$ for $\left.i \neq j\right)$, agent $i$ 's optimal action is characterised by (3.32)-(3.33) and agent $i$ 's expected payoff is:

$$
E_{i}\left[u_{i}^{P}\right]=-\frac{(1+r)^{2}(\alpha+\beta)}{[(1+r) \alpha+\beta]^{2}}-p
$$

In this case agent $i$ does not deviate from the population profile.

When all agents are purchasing and agent $i$ deviates $\left(\nu_{i}=0\right.$ and $\nu_{j}=1$ for $i \neq j$ ), agent $i$ 's optimal action is identical to that in the M-S case and is equal to $y$, as implied by (3.54). Substituting the unit weight on $y$ as optimal action and the average action of a purchasing population into the payoff and taking expectations of the resulting expression yields:

$$
E_{i}\left[u_{i}^{P d}\right]=-\frac{(1+r)^{2}(\alpha+\beta)^{2}}{\alpha[(1+r) \alpha+\beta]^{2}}
$$

Agent $i$ expects to suffer the loss in (3.101) in the event where all agents decide to purchase a private signal, except for agent $i$. In this case agent $i$ deviates from the population profile.

By comparing expressions in (3.100) and (3.101), we derive a value for critical price $p_{\max }$ such that $E_{i}\left[u_{i}^{P}\right]=E_{i}\left[u_{i}^{P d}\right]$. Thus, a purchasing symmetric Nash 
equilibrium (P-NE) exists when:

$$
0<p<\frac{(1+r)^{2} \beta(\alpha+\beta)}{\alpha[(1+r) \alpha+\beta]^{2}}
$$

where $p_{\max } \equiv \frac{(1+r)^{2} \beta(\alpha+\beta)}{\alpha[(1+r) \alpha+\beta]^{2}}$. The above critical value is always positive, strictly decreasing in $\alpha$, strictly increasing in $\beta$ and strictly increasing in $r$. As in the M-S case, higher quality of public information makes agent $i$ more willing to deviate from a purchasing population, and a more precise private signal encourages information acquisition. Higher $r$ means more importance is given to the coordination motive, which, in turn, makes deviating a less attractive strategy.

In order to determine optimal CBT setting in P-NE, we substitute best-response weights from (3.32)-(3.33) into expected welfare in (3.43) to obtain expected equilibrium welfare:

$$
E\left[W^{P}\right]=-\frac{(1+r)^{2}(\alpha+\beta)}{[(1+r) \alpha+\beta]^{2}}-p .
$$

First derivative of (3.103) with respect to the accuracy of the public signal $\alpha$ is positive, thus, maximum CBT is optimal $(\alpha=\gamma)$, and:

$$
E\left[W^{P}\right]_{\alpha=\gamma}=-\frac{(1+r)^{2}(\gamma+\beta)}{[(1+r) \gamma+\beta]^{2}}-p,
$$

is the expected equilibrium outcome under full CBT.

\subsubsection{Non-purchasing equilibria}

As in the M-S case, when no agents are purchasing private information $\left(\nu_{i}=\nu_{j}=0\right.$ for $i \neq j$ ), agent $i$ 's expected payoff is equal to (3.63):

$$
E_{i}\left[u_{i}^{N}\right]=-\frac{1}{\alpha}
$$

where agent $i$ is not deviating from the population profile.

When no agents are purchasing and agent $i$ deviates $\left(\nu_{i}=1\right.$ and $\nu_{j}=0$ for $i \neq j$ ), the optimisation procedure yields an optimal action identical to that in the M-S case and given by (3.67). With this optimal response, agent $i$ 's expected payoff becomes:

$$
E_{i}\left[u_{i}^{N d}\right]=-\frac{1}{\alpha+\beta}-p .
$$

In this case agent $i$ deviates from the non-purchasing population by choosing to purchase $x_{i}$. It should be noted that (3.105) is independent of the coordination 
parameter. This is due to the way the payoff function is formulated, that allows the agent to completely offset the average action term $-r \bar{a}^{N}$ and focus on matching the state variable $\theta$ to best of agent $i$ 's ability. Thus, agent $i$ 's expected loss only depends on the quality of available information and the information cost.

Comparing the expected payoffs in (3.63) and (3.105), we find the condition on information cost that, if met, is consistent with the existence of a non-purchasing symmetric Nash equilibrium (N-NE):

$$
\frac{\beta}{\alpha(\alpha+\beta)}<p
$$

where $p_{\min } \equiv \frac{\beta}{\alpha(\alpha+\beta)}$. The above critical value is always positive, strictly decreasing in $\alpha$, strictly increasing in $\beta$ and independent of the coordination parameter $r$.

Given that, in the absence of policy and information acquisition, the CB commits to full CBT, the expected welfare outcome (and the expected individual payoff) is given by (3.46). Under full CBT, the critical price value that characterises the existence of N-NE becomes:

$$
p_{\min }=\frac{\beta}{\gamma(\gamma+\beta)}
$$

\subsubsection{Equilibrium conditions for P-NE and N-NE}

We can now combine the necessary conditions for existence of the two types of NE. Figure 3.4 demonstrates equilibrium strategies and optimal CBT, where:

$$
\begin{aligned}
p_{\text {min }} & =\frac{\beta}{\gamma(\gamma+\beta)} \\
p_{\text {max }} & =\frac{(1+r)^{2} \beta(\gamma+\beta)}{\gamma[(1+r) \gamma+\beta]^{2}},
\end{aligned}
$$

from (3.102) and (3.107).

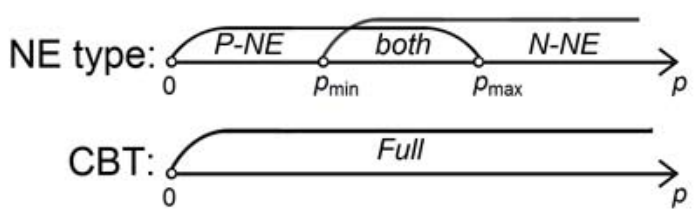

Figure 3.4: Fixed cost. Description of equilibria (J-L)

At lower levels of private information cost $\left(0<p<p_{\min }\right)$, agents find it worthwhile to purchase private signals. Whereas, when information cost is high 
$\left(p_{\max }<p\right)$, there is no private information acquisition in equilibrium. There exists a set of intermediate prices $\left(p_{\min }<p<p_{\max }\right)$, for which both purchasing and nonpurchasing NE exist. Optimal communication policy is independent of information costs, thus, in absence of direct policy intervention, the central bank commits to full transparency invariably.

\subsubsection{Efficient benchmark}

In order to be able to assess the efficiency of equilibria identified above, we now derive an efficient benchmark case for the J-L payoff function. As in the M-S case, we first characterise efficient use of information for a purchasing and a non-purchasing populations, and then derive the critical value of $p$ that defines efficient information acquisition.

We begin with the efficient benchmark for an economy where agents purchase private information. From (3.2), social welfare is given by:

$$
\tilde{W}^{P}=-\int_{0}^{1}\left[\tilde{a}_{i}^{P}-\theta+r\left(\tilde{a}_{i}^{P}-\tilde{\bar{a}}^{P}\right)\right]^{2} d i-p,
$$

where agent $i$ 's efficient response is $\tilde{a}_{i}^{P}=\tilde{\kappa}_{1}^{P} x_{i}+\tilde{\kappa}_{2}^{P} z$ and the average action is $\widetilde{\bar{a}}^{P}=\tilde{\kappa}_{1}^{P} \theta+\tilde{\kappa}_{2}^{P} z$. Taking expectations and maximising the resulting expression with respect to efficient weights yields:

$$
\begin{aligned}
\tilde{\kappa}_{1}^{P} & =\frac{\beta}{\beta+(1+r)^{2} \gamma} \\
\tilde{\kappa}_{2}^{P} & =1-\tilde{\kappa}_{1}^{P} .
\end{aligned}
$$

As opposed to the M-S case, efficient weights in the J-L case depend on the value of the coordination parameter $r$. This is due to the collectively efficient degree being positive, which implies non-neutrality of coordination and, more specifically, a benefit from coordination at the aggregate level. We then substitute the efficient weights back into (3.108) to get the expected efficient welfare outcome for a purchasing economy:

$$
E\left[\tilde{W}^{P}\right]-\frac{(1+r)^{2}}{\beta+(1+r)^{2} \gamma}-p
$$

We now turn to the case of a non-purchasing economy. From (3.2), the social welfare is:

$$
\tilde{W}^{N}=-\int_{0}^{1}\left[\tilde{a}_{i}^{N}-\theta+r\left(\tilde{a}_{i}^{N}-\tilde{\bar{a}}^{N}\right)\right]^{2} d i
$$


where agent $i$ 's efficient response and average action are $\tilde{a}_{i}^{N}=\tilde{\bar{a}}^{N}=\tilde{\kappa}_{2}^{N} z$. Taking unconditional expectations of (3.111) and maximising the resulting expression with respect to $\tilde{\kappa}_{2}^{N}$ yields:

$$
\tilde{\kappa}_{2}^{N}=1
$$

Taking into account that it is efficient for non-purchasing agents to react to the public signal one-to-one, the expected efficient welfare outcome is:

$$
E\left[\tilde{W}^{N}\right]=-\frac{1}{\gamma}
$$

Finally, we derive the critical price value, for which it does not matter whether all agents purchase a private signal or refrain from information acquisition (in both cases the expected welfare outcome is the same). For this purpose we solve $E\left[\tilde{W}^{P}\right]=$ $E\left[\tilde{W}^{N}\right]$ for $p$ and find:

$$
\tilde{p}=\frac{\beta}{\gamma\left[\beta+(1+r)^{2} \gamma\right]}
$$

It should be noted, that under the assumptions of the efficient benchmark, the CB has to impose $p=\tilde{p}$ as the critical price beyond which agents are not to purchase private information. This is due to the fact that imposing efficient use of information does not guarantee efficient information acquisition. This point will be further discussed in the following section.

\subsubsection{Efficiency of equilibria}

We are now in the position to assess the efficiency of equilibria identified for the J-L payoff function in an economy with fixed information costs. As before, we discuss efficiency with reference to three aspects: (i) information use, (ii) information acquisition, and (iii) social welfare outcome. We are comparing two sets of expressions given in Table 3.2 .

When agents acquire private information in equilibrium (P-NE), inefficient use of that information leads to a suboptimal welfare outcome. Inefficient use is characterised by excess weight placed on private information and insufficient weight put on the common signal. This behaviour is consistent with the equilibrium degree of coordination being below the collectively efficient degree of coordination, which is characteristic of the J-L payoff function. On the other hand, when agents do not find it worthwhile to purchase $x_{i}$ (N-NE), information use is efficient, which, in turn, leads to the first-best expected welfare outcome.

As in the M-S case, in equilibrium there exists a price range for which information acquisition is inefficient. The three critical price values relate to each other in the 


\begin{tabular}{|c|c|c|c|c|}
\hline & Equilibrium & & Efficient Benchmark & \\
\hline $\begin{array}{l}E\left[W^{P}\right] \\
\kappa_{1}^{P} \\
\kappa_{2}^{P} \\
\text { Critical } p\end{array}$ & $\begin{array}{l}-\frac{(1+r)^{2}(\gamma+\beta)}{[(1+r) \gamma+\beta]^{2}}-p \\
\frac{\beta}{(1+r) \gamma+\beta} \\
1-\kappa_{1}^{P} \\
p_{\max }=\frac{(1+r)^{2} \beta(\gamma+\beta)}{\gamma[(1+r) \gamma+\beta]^{2}}\end{array}$ & $\begin{array}{r}(3.104) \\
(3.32)\end{array}$ & $\begin{array}{l}-\frac{(1+r)^{2}}{\beta+(1+r)^{2} \gamma}-p \\
\frac{\beta}{\beta+(1+r)^{2} \gamma} \\
1-\tilde{\kappa}_{1}^{P} \\
\tilde{p}=\frac{\beta}{\gamma\left[\beta+(1+r)^{2} \gamma\right]}\end{array}$ & $\begin{array}{l}(3.110) \\
(3.109)\end{array}$ \\
\hline $\begin{array}{l}E\left[W^{N}\right] \\
\kappa_{2}^{N} \\
\text { Critical } p\end{array}$ & $\begin{array}{l}-\frac{1}{\gamma} \\
1 \\
p_{\text {min }}=\frac{\beta}{\gamma(\gamma+\beta)}\end{array}$ & $\begin{array}{r}(3.46) \\
(3.37) \\
(3.106)\end{array}$ & $\begin{array}{l}-\frac{1}{\gamma} \\
1 \\
\tilde{p}=\frac{\beta}{\gamma\left[\beta+(1+r)^{2} \gamma\right]}\end{array}$ & $\begin{array}{l}(3.113) \\
(3.112) \\
(3.114)\end{array}$ \\
\hline
\end{tabular}

Table 3.2: Fixed cost. Equilibrium characterisation versus efficient benchmark (J-L)

following way:

$$
0<\tilde{p}<p_{\min }^{N}<p_{\max }^{P}
$$

This means that information acquisition is in line with the efficient benchmark for very low $(0<p \leq \tilde{p})$ and very high $\left(p_{\max }^{P} \leq p\right)$, but not intermediate prices:

\section{Condition Benchmark Is information acquisition efficient?}

$\begin{array}{cll}0<p \leq \tilde{p} & \mathrm{P} & \text { Yes }(\mathrm{P}-\mathrm{NE}) \\ \tilde{p}<p \leq p_{\min }^{N} & \mathrm{~N} & \text { No }(\mathrm{P}-\mathrm{NE}) \\ p_{\min }^{N}<p<p_{\max }^{P} & \mathrm{~N} & \text { No }(\text { if } \mathrm{P}-\mathrm{NE}), \text { Yes }(\text { if } \mathrm{N}-\mathrm{NE}) \\ p_{\max }^{P} \leq p & \mathrm{~N} & \text { Yes }(\mathrm{N}-\mathrm{NE})\end{array}$

Inefficient information acquisition is caused by the difference between the socially optimal and the equilibrium degrees of coordination. The social value of coordination at the aggregate level exceeds the benefit from coordination as perceived by individual agents. This leads to inefficient use of information, which, in turn, affects agents' strategies about whether or not to purchase a private signal at a given price.

If inefficient use of information drives the values $p_{\max }^{P}$ and $p_{\min }^{N}$ away from the efficient critical price, the question arises whether imposing efficient use ensures efficient information acquisition. To be able to answer this question, we re-derive 
$p_{\max }^{P}$ and $p_{\min }^{N}$ under the assumption that:

$$
\begin{gathered}
\kappa_{1}^{P}=\kappa_{1}^{N d}=\tilde{\kappa}_{1}^{P} \\
\kappa_{2}^{P}=\kappa_{2}^{N d}=\tilde{\kappa}_{2}^{P} \\
\kappa_{2}^{N}=\kappa_{2}^{P d}=\tilde{\kappa}_{2}^{N} .
\end{gathered}
$$

We find that efficient use of information is insufficient to ensure that information acquisition occurs in line with the efficient benchmark. The reason for this is the difference in the way that endogeneity of information acquisition manifests at the individual and aggregate levels. An individual agent is deciding whether or not to deviate from a population of agents that are all either purchasing or not purchasing a private signal. Deviating from the population profile has implications for the coordination term of the expected payoff. Whereas at the level of the economy, the choice is between two types of symmetric equilibria, in which all agents follow the same strategy. There are no additional effects on the coordination term, the latter being non-zero only in P-NE and reflecting the dispersion caused by individual errors in privately acquired information.

\subsubsection{With direct policy intervention}

As in the M-S case, we now introduce direct policy intervention directly into the J-L payoff function. From (3.11), agent $i$ 's payoff is given by:

$$
u_{i}=-\left[a_{i}-\theta-g+r\left(a_{i}-\bar{a}\right)\right]^{2}-\nu_{i} p
$$

where $\nu_{i} \in\{0,1\}$ and $g=\rho z$.

\subsubsection{Purchasing equilibria}

When all agents are acquiring private information $\left(\nu_{i}=\nu_{j}=1\right.$ for $\left.i \neq j\right)$, optimal action is characterised by (3.26)-(3.27). Using that, we can find agent $i$ 's expected payoff, the expression for which is omitted here due to its length.

When all agents are purchasing and agent $i$ deviates $\left(\nu_{i}=0\right.$ and $\nu_{j}=1$ for $\left.i \neq j\right)$, agent $i$ 's optimal action is the same as in the the M-S case and given by (3.82). The expression for agent $i$ 's expected payoff is omitted here, however, it should be noted that the expected payoffs in the two cases - deviating and not deviating - are not the same.

We can now find the values of price $p$ that are consistent with the existence of 
a symmetric Nash equilibrium in which all agents are purchasing a private signal (P-NE):

$$
0<p<\frac{(1+r)^{2} \beta(\alpha+\beta)[(1+\rho) \gamma-\alpha \rho]^{2}}{\alpha[(1+r) \alpha+\beta]^{2} \gamma^{2}},
$$

where $p_{\max } \equiv \frac{(1+r)^{2} \beta(\alpha+\beta)[(1+\rho) \gamma-\alpha \rho]^{2}}{\alpha[(1+r) \alpha+\beta]^{2} \gamma^{2}}$ and is derived by solving $E_{i}\left[u_{i}^{P}\right]=$ $E_{i}\left[u_{i}^{P d}\right]$. The critical value $p_{\max }$ is always positive.

\subsubsection{Welfare, instrument adjustment, and CBT in P-NE}

In order to arrive at the expression for equilibrium expected welfare, we substitute best response weights from (3.26)-(3.27) into (3.41). The obtained expression is omitted here due to its length. We first maximise expected equilibrium welfare with respect to the policy parameter and then determine the optimal CBT regime.

The sign of $\partial E\left[W^{P}\right] / \partial \rho$ is ambiguous. Having found the critical point, we perform the second derivative test that shows that the identified critical point is a maximum. Thus the optimal setting for the policy rule $\rho z$ involves:

$$
\rho^{P}=-\frac{(1+r)^{2}(\alpha+\beta) \gamma}{(1+r) \alpha[(1-r+\beta) \beta+(1+r)(1+\beta+r \beta) \gamma]}
$$

Given optimal policy, expected equilibrium welfare becomes:

$$
E_{i}\left[W^{P}\right]_{\rho=\rho^{P}}=\frac{\rho^{P}}{\gamma}-p
$$

Now we can maximise (3.118) with respect to $\alpha$ to find optimal level of CBT. The sign of the first derivative indicates that $E_{i}\left[W^{P}\right]_{\rho=\rho^{P}}$ is monotonically decreasing in $\alpha$. This means that minimum level of CBT is optimal:

$$
E_{i}\left[W^{P}\right]_{\rho=\rho^{P}, \alpha=0}=-\frac{(1+r)^{2}}{\beta+(1+r)^{2} \gamma}-p
$$

where $\left.\rho^{P}\right|_{\alpha=0}=-\frac{(1+r)^{2} \gamma}{\beta+(1+r)^{2} \gamma}$.

Under zero transparency, agent $i$ has no incentive to deviate from a purchasing population. The CB commits to only partially offsetting the shock $\left(-1<\rho^{P}<0\right)$. A deviator that has access to no signals can only act passively $\left(a_{i}^{P d}=0\right)$ thereby exposing oneself to an undefined expected loss. Agent $i$ is left with no choice but to purchase a private signal for all values of $p$. 
Given optimal policy and zero transparency, a purchasing agent's best response is characterised by:

$$
\begin{aligned}
& \left.\kappa_{1}^{P}\right|_{\rho=\rho^{P}, \alpha=0}=\frac{\beta}{\beta+(1+r)^{2} \gamma} \\
& \left.\kappa_{2}^{P}\right|_{\rho=\rho^{P}, \alpha=0}=0 .
\end{aligned}
$$

Agent $i$ ignores the uninformative public signal and only reacts to the privately acquired $x_{i}$. The optimal weight $\kappa_{1}^{P}$ and the policy parameter $\rho^{P}$ add up to unity, which implies that agent $i$ uses the private signal to offset the remainder of the shock not already offset by direct policy intervention ${ }^{25}$.

\subsubsection{Non-purchasing equilibria}

When no agents are acquiring private information $\left(\nu_{i}=\nu_{j}=0\right.$ for $\left.i \neq j\right)$, agent $i$ 's optimal action is characterised by the best-response weight in (3.36). Given optimal action, agent $i$ 's expected payoff is identical to (3.88) in the M-S case.

When no agents are purchasing and agent $i$ deviates $\left(\nu_{i}=1\right.$ and $\nu_{j}=0$ for $i \neq j$ ), agent $i$ 's optimal action is the same as in the M-S case and given by (3.92). Given optimal action, we can find agent $i$ 's expected payoff:

$$
E_{i}\left[u_{i}^{N d}\right]=-\frac{\gamma^{2}+2(\gamma-\alpha) \gamma \rho+(\gamma-\alpha)(\beta+\gamma) \rho^{2}}{(\alpha+\beta) \gamma^{2}}-p
$$

By comparing (3.88) and (3.121), we can find the values of price $p$ that are consistent with the existence of a symmetric Nash equilibrium in which information acquisition does not occur (N-NE):

$$
\frac{\beta[(1+\rho) \gamma-\alpha \rho]^{2}}{\alpha(\alpha+\beta) \gamma^{2}}<p
$$

where $p_{\min } \equiv \frac{\beta[(1+\rho) \gamma-\alpha \rho]^{2}}{\alpha(\alpha+\beta) \gamma^{2}}$ and $p_{\min }$ solves $E_{i}\left[u_{i}^{N}\right]=E_{i}\left[u_{i}^{N d}\right]$. The above critical value is always positive.

We can now look at the effect optimal choice of policy and CBT have on $p_{\text {min }}$. In a N-NE the $\mathrm{CB}$ commits to $\rho^{N}=-1$, individual agents are passive, and expected equilibrium welfare as well as expected payoffs are given by (3.49). When the CB

\footnotetext{
${ }^{25} \mathrm{As}$ in the M-S case with policy, $\kappa_{1}^{P}$ and $\rho^{P}$ are Bayesian weights.
} 
commits to $\rho=\rho^{N}, p_{\min }$ becomes:

$$
\left.p_{\min }\right|_{\rho=\rho^{N}}=\frac{\alpha \beta}{(\alpha+\beta) \gamma^{2}}
$$

which is monotonically increasing in $\alpha$ and $\beta$, monotonically decreasing in $\gamma$, and independent of $r$. It should be noted that, as in the M-S case, the choice of $\alpha$ is neutral at the aggregate level, yet affects the expected payoff of an agent that considers deviating from a non-purchasing population (and, in turn, affects the value of $\left.p_{\min }\right)$.

\subsubsection{Equilibrium conditions for P-NE and N-NE}

Bringing together the results from the above analysis of P-NE and N-NE, we arrive at the following:

\begin{tabular}{llll} 
Type & Condition & Rule & CBT \\
\hline P-NE & $0<p$ & $\left.\rho^{P}\right|_{\alpha=0}=-\frac{(1+r)^{2} \gamma}{\beta+(1+r)^{2} \gamma}$ & $\alpha=0$ (Zero) \\
N-NE & $\left.p_{\text {min }}\right|_{\rho=\rho^{N}}<p$ & $\rho^{N}=-1$ & $0 \leq \alpha \leq \gamma$ (Any)
\end{tabular}

A P-NE exists for all values of price $p$ and is characterised by the $\mathrm{CB}$ committing to zero CBT and a rule that partially offsets the shock $\left(-1<\rho^{P}<0\right)$. In case of N-NE, the CB commits to a one-to-one instrument adjustment $\left(\rho^{N}=-1\right)$ and can influence the lower boundary of the range, for which N-NE exists, through the setting of $\mathrm{CBT}^{26}$. When P-NE and N-NE are considered separately, the setting of policy for the two types of NE is in conflict. When the possibility of existence of both NE types is assumed in a single economy, further analysis is required to identify optimal setting of $\rho$ and $\alpha$ for every given value of $p$.

The analysis below is similar to that previously carried out for the M-S case, thus, here it is discussed in less detail. Firstly, we work out when a P-NE is socially preferable to a N-NE. For this purpose, we compare the expected welfare outcomes in the two NE types, given optimal policy and CBT that correspond to each type. We, thus, compare the welfare outcomes from (3.49) and (3.119), and find that $E\left[W^{P}\right]_{\rho=\rho^{P}}$ is greater than $E\left[W^{N}\right]_{\rho=\rho^{N}}$ when:

$$
p<\frac{\beta}{\gamma\left[\beta+(1+r)^{2} \gamma\right]}
$$

\footnotetext{
${ }^{26}$ The critical value $p_{\min }$ is equal to zero under zero CBT and $\frac{\beta}{\gamma(\beta+\gamma)}$ under full CBT.
} 
where $p_{\text {dom }} \equiv \frac{\beta}{\gamma\left[\beta+(1+r)^{2} \gamma\right]}$. CB's aim is then to use direct policy intervention and CBT to induce a P-NE for $0<p<p_{\text {dom }}$, and an N-NE otherwise.

Secondly, we evaluate the critical values of $p$ that define the existence of either type of NE under: (i) $\rho=\rho^{P}$ and zero CBT, and (ii) $\rho=\rho^{N}$. The critical values in question are:

$$
\begin{aligned}
& p_{\text {max }}=\frac{(1+r)^{2} \beta(\alpha+\beta)[(1+\rho) \gamma-\alpha \rho]^{2}}{\alpha[(1+r) \alpha+\beta]^{2} \gamma^{2}} \\
& p_{\text {min }}=\frac{\beta[(1+\rho) \gamma-\alpha \rho]^{2}}{\alpha(\alpha+\beta) \gamma^{2}},
\end{aligned}
$$

from (3.116) and (3.122). If the CB commits to $\rho=\rho^{P}$ and zero CBT, the values of both $p_{\max }$ and $p_{\min }$ become infinitely large, meaning that a P-NE exists for all $p$ and a N-NE does not exist at all. Thus, it is sufficient for the CB to choose $\rho^{P}$ and $\alpha=0$ for $0<p<p_{\text {dom }}$ in order to induce a P-NE and prevent a N-NE from existing.

If the $\mathrm{CB}$ commits to $\rho=\rho^{N}$, the critical price values become ${ }^{27}$ :

$$
\begin{aligned}
\left.p_{\text {max }}\right|_{\rho=\rho^{N}} & =\frac{(1+r)^{2} \alpha \beta(\alpha+\beta)}{[(1+r) \alpha+\beta]^{2} \gamma^{2}} \\
\left.p_{\text {min }}\right|_{\rho=\rho^{N}} & =\frac{\alpha \beta}{(\alpha+\beta) \gamma^{2}} .
\end{aligned}
$$

Once $\mathrm{CB}$ commits to a one-to-one policy rule, the exact value of $\alpha$ does not have an effect on the expected welfare outcome in a N-NE. The next step is to choose a value of $\alpha$ such that $\left.p_{\max }\right|_{\rho=\rho^{N}}$ is below $p_{\text {dom. }}$. This ensures that a P-NE does not exist for $p_{\text {dom }}<p$. Zero CBT accomplishes exactly that. Under zero CBT, both critical values $\left.p_{\max }\right|_{\rho=\rho^{N}}$ and $\left.p_{\min }\right|_{\rho=\rho^{N}}$ are equal to zero, which places them below $p_{\text {dom }}$. In other words, if a CB commits to $\rho=\rho^{N}$ and zero CBT, N-NE exist for all values of $p$ and P-NE do not exist at all.

We can now conclude that the CB finds it optimal to set policy in the following way:

\footnotetext{
${ }^{27}$ The value for $\left.p_{\min }\right|_{\rho=\rho^{N}}$ is taken directly from (3.123).
} 


\begin{tabular}{llll} 
Type & Condition & Rule & CBT \\
\hline & & & \\
P-NE & $0<p \leq p_{\text {dom }}$ & $\left.\rho^{P}\right|_{\alpha=0}=-\frac{(1+r)^{2} \gamma}{\beta+(1+r)^{2} \gamma}$ & $\alpha=0$ (Zero) \\
N-NE & $p_{\text {dom }} \leq p$ & $\rho^{N}=-1$ & $\alpha=0$ (Zero)
\end{tabular}

Committing to $\rho=\rho^{P}$ for $0<p<p_{\text {dom }}, \rho=\rho^{N}$ for $p_{\text {dom }}<p$, and zero CBT for all values of $p$ is sufficient to ensure that only the socially preferable type of NE exists for each value of $p$. When $p=p_{\text {dom }}$ it does not matter which of the two values of $\rho$ the $\mathrm{CB}$ commits to, as the expected welfare outcome is the same in P-NE and $\mathrm{N}-\mathrm{NE}^{28}$.

\subsubsection{Efficiency of equilibria}

In order to assess the efficiency of equilibria, we need not derive an efficient benchmark, since the benchmark outcome previously derived for the setting without policy applies to the policy case as well. The relevant expressions are given in Table 3.3.

\begin{tabular}{ll|l} 
Equilibrium & & Efficient Benchmark \\
\hline$E\left[W^{P}\right]_{\rho=\rho^{P}}^{\alpha=0}=-\frac{(1+r)^{2}}{\beta+(1+r)^{2} \gamma}-p$ & $(3.119)$ & $E\left[\tilde{W}^{P}\right]=-\frac{(1+r)^{2}}{\beta+(1+r)^{2} \gamma}-p$ \\
$E\left[W^{N}\right]_{\rho=\rho^{N}}=-\frac{1}{\gamma}$ & $(3.49)$ & $E\left[\tilde{W}^{N}\right]=-\frac{1}{\gamma}$ \\
$p_{\text {dom }}=\frac{\beta}{\gamma\left[\beta+(1+r)^{2} \gamma\right]}$ & $(3.124)$ & $\tilde{p}=\frac{\beta}{\gamma\left[\beta+(1+r)^{2} \gamma\right]}$ \\
$\kappa_{1}^{P}=\frac{\beta}{\beta+(1+r)^{2} \gamma}$ & $(3.120)$ & $\tilde{\kappa}_{1}^{P}=\frac{\beta}{\beta+(1+r)^{2} \gamma}$ \\
$\kappa_{2}^{P}=0,\left.\rho^{P}\right|_{\alpha=0}=-\frac{(1+r)^{2} \gamma}{\beta+(1+r)^{2} \gamma}$ & & $\tilde{\kappa}_{2}^{P}=\frac{(1+r)^{2} \gamma}{\beta+(1+r)^{2} \gamma}$ \\
$\kappa_{2}^{N}=0, \rho^{N}=-1$ & $\tilde{\kappa}_{2}^{N}=1$
\end{tabular}

Table 3.3: Fixed cost. Equilibrium characterisation versus efficient benchmark (J-L with policy)

Expected equilibrium welfare outcomes in both P-NE and N-NE are equal to the efficient outcomes in (3.110) and (3.113). The combination of optimal direct policy intervention and CBT enables the CB to achieve efficient use of information in the economy as well as efficient acquisition of private information by individual agents. Individual agents cannot make use of publicly available information, as it is completely uninformative. However, no informational value is lost due to the CB

\footnotetext{
${ }^{28}$ Due to the social value of private information acquisition being exactly equal to its cost.
} 
using its private signal to offset the shock to the best of its ability.

\subsubsection{Effect of policy on information acquisition}

As in the M-S case, optimally determined setting of CBT on its own is unable to correct the inefficiencies in the acquisition and use of information, for all $p$. Committing to publishing the public signal with maximum quality only partially alleviates the excess weight that individual agents place on their private signals in P-NE. Perceived value of private information being higher than collectively efficient, critical prices that characterise individual agent's strategies for purchasing and not purchasing $x_{i}$ deviate from the efficient value $\tilde{p}$. That said, there exist two price ranges for which information acquisition is in line with the efficient benchmark. The lower price range corresponds to P-NE that are characterised by suboptimal welfare outcomes due to inefficient use of information. The higher price range corresponds to N-NE that are characterised by both first-best welfare outcome and efficient use of the public signal.

Introduction of direct policy intervention allows the CB to influence the way information is used as well as its perceived value. Under zero CBT, agent $i$ 's decision of whether or not to purchase private information solely depends on the degree to which the $\mathrm{CB}$ is offsetting the shock. Partial instrument adjustment means that agents have no choice but to acquire $x_{i}$ at any cost in order to match the portion of the shock not offset by the rule ${ }^{29}$. Full instrument adjustment removes the incentive to purchase private information entirely. This power allows the CB to construct a collectively efficient strategy of choosing the value of the policy parameter $\rho$ based on the actual value of $p$ to induce either a P-NE or a N-NE. By committing to a partial rule for $0<p \leq \tilde{p}$ and a one-to-one rule for $\tilde{p} \leq p$, the $\mathrm{CB}$ is able to achieve efficient information acquisition.

By committing to a one-to-one rule when it is optimal to do so, the CB achieves both efficient information acquisition as well as efficient use of its private signal $z$. This results in all N-NE expected welfare outcomes being the best attainable. When committing to partially offsetting the shock and inducing a P-NE, the CB is able to choose any value of $\rho$ that is not equal to minus unity, which creates further scope for influencing the way acquired private information is used. By setting $\rho=\rho^{*}$, the $\mathrm{CB}$ encourages agents to align their best response with the collectively efficient weight response to private information. Since the CB uses its private signal in its instrument adjustment, no informational value is lost in the economy and first-best

\footnotetext{
${ }^{29} \mathrm{As}$ noted before, this result is a direct consequence of our assumptions about the variance of the state variable.
} 
expected welfare outcomes are achieved in all P-NE. This result constitutes Proposition 3.2 .

Proposition 3.2: If agents aim to maximise a J-L payoff function and information cost $p$ is fixed, commitment to an optimally designed monetary policy rule in combination with optimal level of transparency with respect to the central bank's private signal achieve efficient use of information, efficient information acquisition, as well as the first-best welfare outcome for all values of $p$.

\subsection{Linear Cost: Morris-Shin Payoff Function}

\subsubsection{In the absence of policy}

In this Section we look at model variations with a linear information cost component and M-S payoff. From (3.1), agent $i$ 's payoff function is given by:

$$
u_{i}=-\left(a_{i}-\theta\right)^{2}-r\left(L_{i}-\bar{L}\right)-\beta_{i} p
$$

where $\beta_{i}$ is agent $i$ 's individual precision of private signal $x_{i}$, and $p$ the cost of private information per unit of precision $\beta_{i}$.

In order to characterise information acquisition in equilibrium, we use backward induction and assume that agent $i$ chooses private signal precision $\beta_{i}$ while taking precision $\beta$ chosen by other agents as given. We proceed to work out the value of $\beta$ that corresponds to a symmetric equilibrium solution. Having done that, we derive a critical price $p$ value below which individual agents find it optimal to choose a positive value of $\beta$. Said critical value of $p$ defines the information price ranges for which either P-NE or N-NE exist. We then look at what effect CBT has on information acquisition.

\subsubsection{Private information acquisition}

Given agent $i$ 's best response under the assumption that potentially $\beta_{i} \neq \beta$, which is identified in (3.30)-(3.31), as well as the best response weights of other agents in (3.32)-(3.33), we can move backward studying agent $i$ 's choice of $\beta_{i}$. For this purpose we take unconditional expectation of the payoff in (3.21), substitute best responses of agent $i$ and other agents into the resulting expression, and, finally, maximise the expected equilibrium payoff with respect to the quality of information $\beta_{i}$. We obtain two roots - one is negative for all parameter values and the other is positive for some combinations of parameter values. We can ignore the negative 
root as it is inadmissible. The remaining root is given by:

$$
\beta_{i}=\frac{(\alpha+\beta) \sqrt{p(1+r)}}{p[(1+r) \alpha+\beta]}-\alpha .
$$

The above expression is increasing in $\beta$ for $0<\beta$. Agents' decisions about the quality of private information, as represented by $\beta_{i}$ and $\beta$, are, in fact, strategic complements (Colombo and Femminis, 2008; Hellwig and Veldkamp, 2009). If agents, other than agent $i$, choose to purchase private information of higher quality (i.e. choose a higher value of $\beta$ ), agent $i$ suffers a greater coordination loss unless $\beta_{i}$ is increased accordingly. Furthermore, it is optimal for agent $i$ to increase $\beta_{i}$ until it is equal to the currently prevailing $\beta$. From an algebraic point of view, it is apparent that expression (3.126) does not depend on any variables that are specific to agent $i$, which is in line with the equilibrium being symmetric and $\beta_{i}=\beta$. Solving (3.126) with $\beta_{i}=\beta$ yields:

$$
\beta=\sqrt{\frac{1+r}{p}}-(1+r) \alpha .
$$

Unsurprisingly, the quality of purchased information is inversely related to both the marginal cost of acquiring information $p$ as well as the quality of publicly available announcement $\alpha \cdot{ }^{30} \mathrm{~A}$ higher price per unit of precision and higher quality of information from an alternative (and free) source discourage agents from investing in private information acquisition. An increase in price per unit of precision $p$, ceteris paribus, raises the cost of purchasing $x_{i}$ with $\beta$ quality, while the net benefit ${ }^{31}$ of acquiring $x_{i}$ with $\beta$ quality remains unchanged. Agent $i$ finds it optimal to lower the value of $\beta$ until the balance between marginal benefit and marginal cost is restored. As far as the effect of changes in $\alpha$ is concerned, the qualities of signals $x_{i}$ and $y$ are strategic substitutes: an increase in precision $\alpha$ has a crowding-out effect on private information acquisition (Colombo and Femminis, 2008). The direct effect of an increase in $\alpha$ works through the combined accuracy ${ }^{32}$ of available information. When the quality of the free public signal increases, this implies a higher information accuracy. By choosing to decrease investment in private information, agents have access to information of the same combined accuracy as they did prior to the increase in $\alpha$, but now at a lower cost.

\footnotetext{
${ }^{30}$ It should be noted that the coefficient on $\alpha$ in (3.127) is consistent with the general expression for endogenous precision of private information derived in Colombo et al. (2014).

${ }^{31}$ The sum of all effects that choosing $\beta$ has on the different components of agent $i$ 's expected payoff, excluding information cost.

${ }^{32}$ Accuracy of available information is the precision of agents' forecasts about $\theta$ (i.e. the inverse of the variance of forecast errors about $\theta$ ) (Angeletos and Pavan, 2007a). In the current setting accuracy is simply given by $\alpha+\beta$. See Appendix C.5
} 
The indirect crowding-out effect of an increase in $\alpha$ on the optimally chosen values of $\beta_{i}$ and $\beta$ works through its effect on the incentive to coordinate. A higher $\alpha$ implies higher commonality ${ }^{33}$ of available information, which, in turn, means more favourable conditions for coordination. With a higher incentive to coordinate, agents put more weight on $y$ in forecasting other's actions, thereby the perceived value of more precise private information is reduced. This indirect effect grows in strength as the importance of coordination to individual agents (as represented by $r$ ) increases, which explains the presence of $(1+r)$ coefficient in the negative term ${ }^{34}$ on the righthand side of (3.127). The presence of $\sqrt{1+r}$ in the positive term reflects the benefit that a higher $\beta$ has for the alignment of agent $i$ 's own action with the actions of others. Namely, a private signal of a higher quality allows agent $i$ to better forecast actions of other agents. This leads to a reduction in loss from agent's own action's dispersion around the aggregate action. The net effect of a change in $r$ on the optimal value of $\beta$ is ambiguous for all $0<\beta$.

The root identified in (3.127) is positive and a symmetric equilibrium exists for:

$$
0<p<\frac{1}{(1+r) \alpha^{2}}
$$

From (3.128), we define:

$$
p_{1} \equiv \frac{1}{(1+r) \alpha^{2}}
$$

If the condition in (3.128) is not met, agents choose zero quality of private information which is equivalent to no information acquisition. The critical price value $p_{1}$ is monotonically falling in the strength of the coordination motive. This stems from the fact that private information acquisition is perceived by agent $i$ to have less value as coordination of actions grows in importance relative to aligning one's action with the shock. Assume, for the moment, that actual price is equal to the critical value $\left(p=p_{1}\right)$ and, therefore, too high for agents to find private information acquisition worthwhile. If the coordination motive were to get marginally weaker (a marginal reduction in $r$ ), ceteris paribus, the incentive to coordinate would be reduced and the crowing-out effect of public information on private information would weaken. Agents would find it worthwhile to choose a marginally higher quality of $x_{i}$, meaning that at price $p$ (and with a lower $r$ ) the symmetric equilibrium would be characterised by a positive $\beta$. In other words, the critical value of price (the new

\footnotetext{
${ }^{33}$ Commonality is the correlation of forecast errors across agents (Angeletos and Pavan, 2007a). In the current setting, commonality is given by $\frac{\alpha}{\alpha+\beta}$. See Appendix C.5

${ }^{34}$ The coordination parameter is not present in the positive term of the solution for $\beta$ presented in Angeletos and Pavan (2007a). Appearance of $r$ in the positive term in (3.127) is a result of the way coordination enters the re-formulated payoff function.
} 
$p_{1}$ ) at which information acquisition ceases to take place would now be greater than $p$, because quality of private information would have more social value.

\subsubsection{Central bank transparency}

At the aggregate level, we substitute agents $i$ 's best response from (3.32)-(3.33) into expected welfare in (3.42):

$$
E[W]=-\frac{\beta}{[(1+r) \alpha+\beta]^{2}}-\frac{(1+r)^{2} \alpha}{[(1+r) \alpha+\beta]^{2}}-\beta p
$$

where $0<p<p_{1}$. As shown in Appendix C.3, the three right-hand side terms in the expressions for expected welfare correspond to loss from dispersion, loss from volatility, and the cost of private information acquisition. Dispersion is the loss associated with the idiosyncrasy of privately purchased information ${ }^{35}$. The volatility term reflects the misalignment of average action with the state variable, which is primarily $^{36}$ due to the limited quality of publicly available information.

For the case where agents find it worthwhile to acquire private information, substituting the solution for $\beta$ from (3.127) into (3.130), allows to account for the indirect effect of changes in $\alpha$ on expected welfare via the crowding-out:

$$
E[W]=-\left[\sqrt{\frac{p}{1+r}}-\alpha p\right]-(1+r) \alpha p-[\sqrt{(1+r) p}-(1+r) \alpha p]
$$

where the first term in square brackets, the second term, and the third term in square brackets remain to be dispersion, volatility, and information cost, respectively. An increase in $\alpha$ affects dispersion through crowding-out of $\beta$ as well as through $\kappa_{1}$. A lower value of $\beta$ directly ${ }^{37}$ translates into higher variance of agents' actions relative to the average action across the population; however, it also means a decrease in the weight put on $x_{i}$, which, in turn, decreases the dispersion. The net effect on the first term is a reduction in loss from dispersion. Volatility loss is strictly increasing in $\alpha$, whereas both dispersion loss and information cost are strictly decreasing in $\alpha$. An improvement in the quality of public information, on the one hand, reduces volatility directly, and, on the other hand, increases volatility indirectly through a higher reliance on the public signal (a higher $\kappa_{2}$ ). The net effect on the second term is a greater loss from volatility. As far at the third term is concerned, information cost gets smaller as $\alpha$ increases due to agents choosing to purchase $x_{i}$ of a lower quality.

\footnotetext{
${ }^{35}$ Quality of public information affects dispersion indirectly through the response coefficient $\kappa_{1}$. ${ }^{36}$ Quality of private information affects volatility indirectly through the response coefficient $\kappa_{2}$.

${ }^{37}$ Controlling for the indirect effect working through $\kappa_{1}$.
} 
In the case of both dispersion and volatility, the secondary effect of a change in $\alpha$ that works through the change in optimal weights $\kappa_{1}$ and $\kappa_{2}$ fully offsets the direct effect. This is due to the effect on weights being amplified by the crowding-out of $\beta$, a tertiary effect. The net welfare effect is strictly positive, which leads to optimality of full CBT:

$$
E\left[W^{P}\right]_{\alpha=\gamma}=p\left(\gamma-\frac{2+r}{\sqrt{(1+r) p}}\right),
$$

where $P$ stands for purchasing equilibrium.

If the cost of information is too high for private information acquisition to happen, namely, if price $p$ is equal to or exceeds the critical value $p_{1}$ leading to $\beta=0$ being agent $i$ 's optimal choice, expected welfare expression is given by (3.46). In the absence of direct policy intervention the CB commits to full CBT, since an increase in the quality of $y$ has a strictly positive effect on expected welfare ${ }^{38}$.

The above solution and results are equivalent to those in Colombo and Femminis (2008) and are presented in this Section in full due to the difference in notation and the formulation of the payoff function, the latter being superficial and not affecting the results qualitatively ${ }^{39}$. Perhaps, the most notable aspect of this solution is the fact that introducing a linear cost structure prevents the occurrence of the standard Morris-Shin result, i.e. the existence of a set of parameter combinations, for which marginally increasing CBT is detrimental to social welfare. It has to do with the linearity of the cost structure and not the introduction of private information acquisition as such, considering that the standard Morris-Shin result is still present in the fixed cost scenario. Endogeneity of the value of $\beta$, as opposed to the mere choice of whether or not to purchase $x_{i}$ of an exogenously given $\beta$ quality, creates a crowding-out effect of public information on private information, thereby increasing the social value of the former. In order to shed more light on why full CBT remains optimal in $\mathrm{P}-\mathrm{NE}$ for all $0<p<p_{1}$ we need to introduce efficiency considerations.

\subsubsection{Efficient benchmark}

Having arrived at an equilibrium solution, we turn to deriving an efficient benchmark solution for this model setup. We take expectations of the welfare equation in (3.38)

\footnotetext{
${ }^{38}$ Furthermore, given a N-NE that exists for some $\alpha<\gamma$ and $\left.p_{1}\right|_{\alpha<\gamma}<p$, adjustment of $\alpha$ to its maximum value $\gamma$ in line with a full CBT policy does not disrupt the N-NE, as the condition $\left.p_{1}\right|_{\alpha=\gamma}<p$ continues to hold.

${ }^{39}$ Arguably, the main difference between the models discussed here and in Colombo and Femminis (2008) is that we assume zero cost of public information.
} 
and impose full CBT from the outset ${ }^{40}$ :

$$
E[W]=-\frac{\kappa_{1}^{2}}{\beta}-\frac{\kappa_{2}^{2}}{\gamma}-\beta p
$$

In order to characterise efficient use of information by the agents, we maximise (3.133) with respect to response weights $\kappa_{1}$ and $\kappa_{2}$. Given private information acquisition is taking place $(0<\beta)$, it is collectively efficient for agents to prioritise information proportionately to its quality:

$$
\begin{aligned}
\tilde{\kappa}_{1}^{P} & =\frac{\beta}{\beta+\gamma} \\
\tilde{\kappa}_{2}^{P} & =1-\tilde{\kappa}_{1}^{P} .
\end{aligned}
$$

Namely, $\tilde{\kappa}_{1}^{P}$ and $\tilde{\kappa}_{2}^{P}$ are the Bayesian weights applied to signals $x_{i}$ and $z$ to form a forecast of $E_{i}[\theta]$. The coordination parameter $r$ does not appear in the pair of efficient weights due to the fact that the 'beauty-contest' motive represented by $r\left(L_{i}-\bar{L}\right)$ is absent from the welfare expression. With information being used efficiently, expected welfare outcome becomes:

$$
E[W]_{\kappa_{1}=\tilde{\kappa}_{1}^{P}}=-\frac{1}{\beta+\gamma}-\beta p
$$

Expression (3.136) is maximised for ${ }^{41}$ :

$$
\tilde{\beta}=\frac{1}{\sqrt{p}}-\gamma
$$

which is positive for $0<p<\frac{1}{\gamma^{2}} \cdot{ }^{42}$ In order to be able to refer to the condition for positivity of $\beta$ more conveniently in our analysis, we introduce:

$$
\tilde{p} \equiv \frac{1}{\gamma^{2}}
$$

Whenever actual price is equal to or exceeds the critical value $\tilde{p}$, it is socially optimal ${ }^{43}$ for agents to opt out of acquiring private signals. The purchasing and non-

\footnotetext{
${ }^{40}$ Assumed distribution of the state variable $\theta$ implies that $\kappa_{1}=1-\kappa_{2}$ is required for efficiency. This eliminates the term in the variance of $\theta$ from the expression.

${ }^{41}$ Optimisation yields another root that is negative for all values of $p$ and $\gamma$.

${ }^{42}$ It should be noted that the coefficient on $\gamma$ in (3.137) is consistent with the general expression for welfare-maximising precision of private information derived in Colombo et al. (2014).

${ }^{43}$ For $\tilde{p} \leq p$, expected welfare is decreasing in quality of $x_{i}$ over the range $0<\beta$.
} 
purchasing efficient outcomes are:

$$
\begin{aligned}
& E\left[\tilde{W}^{P}\right]=\gamma p-2 \sqrt{p} \\
& E\left[\tilde{W}^{N}\right]=-\frac{1}{\gamma},
\end{aligned}
$$

respectively.

\subsubsection{Efficiency of equilibria}

We are now in the position to evaluate the efficiency of information use and acquisition of the earlier obtained equilibrium solution. Comparison of critical price values $p_{1}$ and $\tilde{p}$ - from (3.129) and (3.137), respectively - reveals that information acquisition ceases to take place at lower values of actual price. As Figure 3.5 shows, when $p_{1} \leq p<\tilde{p}$, in equilibrium agents are discouraged from purchasing a private signal, whereas it is still socially efficient to choose a positive $\beta$ in the benchmark scenario. It is the positive value of coordination from agent $i$ 's perspective (and zero

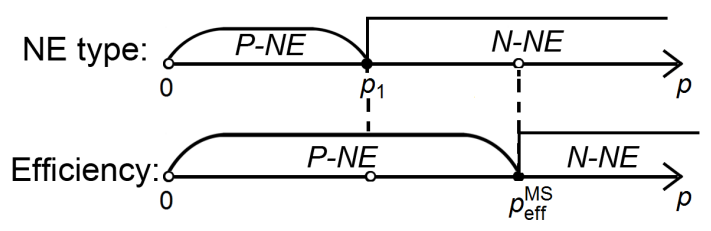

Figure 3.5: Linear cost. Equilibrium and efficient information acquisition (M-S)

value of coordination at the aggregate level) that drives the wedge between the two values of $p$. In line with characteristics of the M-S payoff function, in P-NE individual agents place an inefficiently low weight on privately acquired signals $\left(\kappa_{1}<\tilde{\kappa}_{1}^{P}\right)$. Inefficient use of information factors into agent $i$ 's choice of $\beta$, which, in turn, leads to inefficient signal acquisition.

The way in which the coordination parameter $r$ enters the equilibrium solution for $\beta$ makes said $\beta$-value more responsive to changes in information cost. This is evident from the absolute value of the first derivative of $\beta$ with respect to $p$ in equilibrium being higher than in the benchmark case. In other words, individual agents over-purchase information quality when the cost is low and under-purchase when the cost is high (yet not high enough for acquisition to stop altogether). More specifically, when $0<p<\frac{2+r-2 \sqrt{1+r}}{r^{2} \gamma^{2}}$, agents buy signals of inefficiently high quality. When $\frac{2+r-2 \sqrt{1+r}}{r^{2} \gamma^{2}}<p<p_{1}$, agents buy signals of inefficiently low 
quality. When $p=\frac{2+r-2 \sqrt{1+r}}{r^{2} \gamma^{2}}$, agents buy signals of efficient quality, but these signals are used inefficiently $\left(\kappa_{1}<\tilde{\kappa}_{1}^{P}\right)$. Thus, for all but one value of actual price, the coordination motive prevents agents from making collectively efficient decisions about private information acquisition in $\mathrm{P}-\mathrm{NE}$.

The original Morris-Shin result of negative social value of public information appears in settings with exogenously given quality of private signals. As shown in the fixed cost section above, the result is still present when information acquisition is introduced in the form of a fixed fee for a signal with exogenously given quality. As briefly mentioned above, in the current setting with endogenously chosen $\beta$ and a linear cost structure, full CBT is optimal in all P-NE. Endogeneity of private information in a 'beauty-contest' model, such as the one discussed here, leads to invariably positive social value of public information (Colombo et al., 2014). As Colombo et al. explain, when agents purchase $x_{i}$ with inefficiently high quality, the crowding-out effect of $\alpha$ on $\beta$ increases the social value of public information. By encouraging agents to choose a lower value of $\beta$, an increase in the level of CBT can "boost welfare when the costs saved in the acquisition of private information more than compensate for the increase in non-fundamental volatility relative to dispersion" (Colombo et al., 2014, p. 1455).

To conclude, expected equilibrium outcome is efficient for $\tilde{p} \leq p$ and inefficient otherwise. In other words, equilibrium information acquisition and use are socially optimal when no private information is acquired and the actual price corresponds to a N-NE in the efficient benchmark scenario.

\subsubsection{With direct policy intervention}

We now introduce direct policy intervention into the above model variation and repeat the analysis. With policy present, agent $i$ 's payoff function is given by (3.10):

$$
u_{i}=-\left(a_{i}-\theta-g\right)^{2}-r\left(L_{i}-\bar{L}\right)-\beta_{i} p,
$$

where $g=\rho z$.

\subsubsection{Purchasing equilibria: private information acquisition}

In order to arrive at agent $i$ 's choice of private information quality, we take expectation of the payoff in (3.10), substitute optimal action weights from (3.26)-(3.27) and (3.28)-(3.29), and maximise the resulting expression with respect to $\beta_{i}$. We obtain 
two roots neither of which depend on any variable specific to agent $i$, thus, we set the roots equal to $\beta$ and solve the equations with respect to it. We obtain three critical points, one of which is zero if (and only if) $\alpha=0$ and negative for all other parameter values, one always negative, and one only positive for certain parameter value combinations. The relevant critical point is:

$$
\beta=\frac{\sqrt{p(1+r)(\gamma-\alpha \rho+\gamma \rho)^{2}}}{p \gamma}-(1+r) \alpha
$$

which includes a term $(\gamma-\alpha \rho+\gamma \rho)$ under the square root sign that (if unrestricted) can be positive, zero or negative. First, we restrict $\rho$ to not being equal to $-\frac{\gamma}{\gamma-\alpha}$, which collapses to $\rho$ not being equal to -1 under zero CBT (when $\alpha=0$ ). Otherwise, the term under the square root turns to zero and $\beta$ is strictly negative. An obvious consequence of allowing $\beta$ to be endogenously determined is non-neutrality of policy with respect to the choice of $\beta$. A closer look at expression (3.142) reveals that an incremental increase in $\rho$ results in agents purchasing private signals with lower quality (lower $\beta$ ) if $\rho<-\frac{\gamma}{\gamma-\alpha}$. The opposite is true if $-\frac{\gamma}{\gamma-\alpha}<\rho$. We proceed by looking closer at the parameter value combinations for which the critical point is positive and split them into separate cases based on $\mathrm{CBT}^{44}$ settings, as shown in Table 3.4 .

\begin{tabular}{l|l|l|l|l} 
Case & CBT & Policy & Cost & $x_{i}$ quality \\
\hline 1 & Zero & $\rho \neq-1$ & $0<p$ & $\beta=\frac{\sqrt{p(1+r)(1+\rho)^{2}}}{p}$ \\
2 & Int. & $\rho \neq-\frac{\gamma}{\gamma-\alpha}$ & $p<p_{2}$ & $\beta=\frac{\sqrt{p(1+r)(\gamma-\alpha \rho+\gamma \rho)^{2}}}{p \gamma}-(1+r) \alpha$ \\
3 & Full & - & $p<p_{2}$ & $\beta=\frac{\sqrt{p(1+r)})}{p}-(1+r) \gamma$
\end{tabular}

Table 3.4: Linear cost. Conditions for a positive $\beta$ (M-S with policy)

The critical value of $p$ in Table 3.4 is defined as:

$$
p_{2} \equiv \frac{(\gamma-\alpha \rho+\gamma \rho)^{2}}{(1+r) \alpha^{2} \gamma^{2}}
$$

Whenever parameter value combinations lie outside the above three cases, the critical point is non-positive and the expected payoff is no longer maximised. In that case it is optimal for agents to purchase signal $x_{i}$ with zero quality which is equivalent to not purchasing $x_{i}$ at all.

\footnotetext{
${ }^{44}$ Zero, Int., and Full correspond to $\alpha=0,0<\alpha<\gamma$, and $\alpha=\gamma$, respectively.
} 
The payoff-maximising value of $\beta$ is defined by the assumptions we make about the state variable $\theta$. Specifically, we assume that $\theta$ is drawn from an improper uniform prior on the real line, which, in turn, implies that variance of $\theta$ is not defined. Whenever the shock is not fully offset by policy, individual agents have to match the remainder of the shock to avoid suffering a higher expected loss. In order to do that, agents need access to information about $\theta$ : public, private, or both. In absence of commonly available information, it is worthwhile for the agents to acquire private signals, since the payoff from doing so is always better than the payoff when there is no private information. Thus, under zero CBT, any value of the policy parameter $\rho$, apart from $\rho=-1$, creates an incentive for agents to acquire some private information, no matter the cost. This principle is illustrated by Case 1 above, where zero CBT, $\rho \neq-1$, and all values of $p$ are associated with a positive value of $\beta$. On the other hand, when the $\mathrm{CB}$ commits to offsetting the shock oneto-one $(\rho=-1)$, agents have no incentive to invest in costly information, as there is no need for them to deviate from a passive action strategy.

\subsubsection{Purchasing equilibria: instrument adjustment and CBT}

We will now look at the optimisation problem faced by the $\mathrm{CB}$, starting with the choice of the policy parameter $\rho$ so as to maximise expected equilibrium welfare. For this purpose, we substitute the best response weights from (3.26)-(3.27) into expected welfare in (3.40). We then substitute the value of the positive $\beta$-root and maximise the resulting welfare expression with respect to $\rho$. Given the critical point value of $\beta$ from (3.142), the expected equilibrium welfare is equal to:

$$
E\left[W^{P}\right]_{\beta=\beta^{*}}=\alpha p+\frac{\alpha \rho^{2}}{\gamma^{2}}-\frac{\rho^{2}}{\gamma}-\frac{(2+r) \sqrt{(1+r) p}}{(1+r) \gamma} \cdot|\gamma+(\gamma-\alpha) \rho| .
$$

Expression (3.144) is maximised for ${ }^{45}$ :

$$
\rho^{*}=-\frac{(2+r) \gamma \sqrt{p}}{2 \sqrt{1+r}}
$$

The relationship between $\rho^{*}$ and $\beta$ from (3.142) is such that a weakening in the policy response (an increase in $\rho$ or, equivalently, a decrease in $|\rho|$ ) leads to agents purchasing information of higher quality. It should be noted, that expression (3.145) appears to be independent of the level of CBT chosen by the policymaker. This is due to the fact that (3.145) is only one of the two ways $\rho^{*}$ can be expressed. Rearranging the expression for optimal $\beta$ (3.142) so as to express the price $p$ as a function of the

\footnotetext{
${ }^{45}$ The optimisation procedure yields a positive root as well, however, the positive root corresponds to a lower level of expected welfare and, thus, is dominated by the negative solution.
} 
remaining variables and substituting the resulting expression into (3.145) yields:

$$
\rho^{*}=-\frac{(2+r) \gamma}{(\alpha+\gamma) r+2(\beta+\gamma)}
$$

Once the $\mathrm{CB}$ commits to an instrument adjustment rule defined by $\rho=\rho^{*}$, the conditions for existence of a P-NE become more narrow. In particular, a P-NE exists for:

$$
-\frac{\gamma}{\gamma-\alpha}<\rho<0 \wedge 0<p<p_{3}
$$

where $p_{3} \equiv \frac{4(1+r)}{[2 \gamma+r(\alpha+\gamma)]^{2}}$.

Having established the price range for which a P-NE exist, we turn to determining the effect of changes in CBT on the expected equilibrium outcome. Given $\rho=\rho^{*}$ from (3.145), equilibrium welfare in (3.144) becomes:

$$
E\left[W^{P}\right]_{\beta=\beta^{*}, \rho=\rho^{*}}=p \gamma+\frac{p r^{2}(\gamma-\alpha)}{4(1+r)}-\frac{(2+r) \sqrt{(1+r) p}}{1+r}
$$

Expression (3.148) is monotonically decreasing in $\alpha$, which suggests that it is optimal for the $\mathrm{CB}$ to choose zero CBT. If the $\mathrm{CB}$, accordingly, commits to not releasing a public signal and the per-unit cost of information lies in the range ${ }^{46} 0<p<p_{4}$, where:

$$
p_{4} \equiv \frac{4(1+r)}{(2+r)^{2} \gamma^{2}}
$$

agents find it worthwhile to purchase private information with accuracy equal to:

$$
\beta=\frac{\sqrt{p(1+r)}}{p}-\frac{(2+r) \gamma}{2}
$$

This leads to the following expected welfare outcome:

$$
E\left[W^{P}\right]_{\alpha=0}=-\frac{(2+r) \sqrt{p}[4 \sqrt{1+r}-(2+r) \gamma \sqrt{p}]}{4(1+r)} .
$$

The above expression is monotonically decreasing in the coordination parameter $r$, and monotonically increasing in the quality of CB's private information $\gamma$.

Looking closer at $p_{4}$ from (3.149) and comparing it to $p_{3}$ reveals that $p_{3}$ is strictly lower than $p_{4}$ for any positive quality of the public signal $(0<\alpha)$. Figure 3.6 shows that the price range for which private information acquisition takes place expands as the CB lowers the quality of its public signal from some positive $\alpha$ to zero. This

\footnotetext{
${ }^{46}$ From (3.147), derived by setting $\alpha=0$.
} 
corresponds to the value of $p_{3}$ approaching that of $p_{4}$ and eventually becoming equal to $p_{4}$.

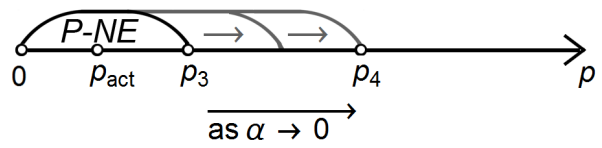

Figure 3.6: Linear cost. Effect of changes in CBT on the existence of P-NE (M-S with policy)

A related question is what the above relationship between the critical price $p_{3}$ and CBT implies for pre-existing equilibria when the $\mathrm{CB}$ decides to adjust some intermediate level of CBT to the optimal zero level. Suppose an equilibrium exists at an actual level of price $p$ that is equal to $p_{3}$ which, in turn, corresponds to some positive $\alpha$. At $p=p_{3}$ individual agents are indifferent between purchasing and not purchasing $x_{i}$. If the $\mathrm{CB}$ marginally lowers the level of CBT, the new value of the critical price $p_{3}$ is now above the actual price $p$. Agents now strictly prefer to purchase private information at price $p$ and the pre-existing equilibrium is maintained. The same applies for any initial $p$ such that $p \leq p_{3}$ and any lowering of $\alpha$ by the CB. Thus, any adjustment of CBT to its optimal level does not disrupt preexisting purchasing equilibria; and, considering that expected equilibrium welfare is decreasing in $\alpha$, it is also preferable for the $\mathrm{CB}$ to do so.

\subsubsection{Non-purchasing equilibria}

Having characterised the P-NE for this model setup, we turn to the conditions for existence of N-NE. A N-NE exists if it is optimal for individual agents to not purchase private information, which in the current setting is equivalent to choosing a non-positive precision $\beta$. From (3.142), the conditions for optimally chosen quality of $x_{i}$ to be non-positive $(\beta \leq 0)$ is given in Table 3.5.

\begin{tabular}{l|l|l|l} 
Case & CBT & Policy & Cost \\
\hline 1 & Zero & $\rho=-1$ & $0<p$ \\
2 & Int. & $\rho=-\frac{\gamma}{\gamma-\alpha}$ & $0<p$ \\
3 & Int. & $\rho \neq-\frac{\gamma}{\gamma-\alpha}$ & $p_{2} \leq p$ \\
4 & Full & - & $p_{2} \leq p$
\end{tabular}

Table 3.5: Linear cost. Conditions for a non-positive $\beta$ (M-S with policy) 
The expression for $p_{2}$ used in Table 3.5 appears in (3.143) and is given by:

$$
p_{2}=\frac{(\gamma-\alpha \rho+\gamma \rho)^{2}}{(1+r) \alpha^{2} \gamma^{2}}
$$

Given the absence of private information acquisition, the CB seeks to commit to a one-to-one policy rule $\left(\rho^{N}=-1\right)$. Expected equilibrium welfare is then given by (3.49), and the conditions for existence of N-NE in Table 3.5 become:

\begin{tabular}{l|l|l} 
Case & CBT & Cost \\
\hline 1 & Zero & $0<p$ \\
2 & Int. & $p_{5} \leq p$
\end{tabular}

where $p_{5} \equiv \frac{1}{(1+r) \gamma^{2}}$.

\subsubsection{Inducing a purchasing equilibrium}

Upon inspection of critical price values $p_{4}$ and $p_{5}$, we can see that there exists a price ranges:

$$
p_{5}<p<p_{4},
$$

for which both types of NE potentially exist. By committing to only partially offsetting the shock and not releasing any public information, the CB is able to induce a P-NE over a price range, for which a one-to-one policy rule ensures the existence of N-NE. The question then arises whether the CB finds it collectively efficient to do so. In order to answer this question, we compare expected equilibrium welfare outcomes corresponding to P-NE and N-NE over the price range in question. The comparison of expressions (3.148) and (3.49) reveals that, indeed, the CB finds it optimal to induce a P-NE whenever the information cost is below $p_{4}$. Thus, the CB's strategy can be summarised in the following way:

$$
\left\{\begin{array}{l}
\text { if } p<p_{4}, \quad \rho=\rho^{*} \wedge \alpha=0(\mathrm{P}-\mathrm{NE}) \\
\text { if } p_{4}<p, \quad \rho=-1(\mathrm{~N}-\mathrm{NE})
\end{array}\right.
$$

where $\rho^{*}$ refers to $(3.145)$. The top panel in Figure 3.7 shows the range of prices for which a P-NE exists, given $\rho=\rho^{*}$ and $\alpha=0$. The middle panel illustrates the existence of N-NE, given $\rho=-1$. Lastly, the bottom panel illustrates the combination strategy that the CB finds optimal and corresponding equilibrium outcomes.

It should be noted that a P-NE cannot be induced for any $p$ that is higher or equal to $p_{4}$. N-NE exist with certainty for $p_{4} \leq p$ and are the only type of outcome that is realised over this price range. The lower bound of the price range in question adjusts downward as the quality of CB's private information improves ( $\gamma$ increases) 


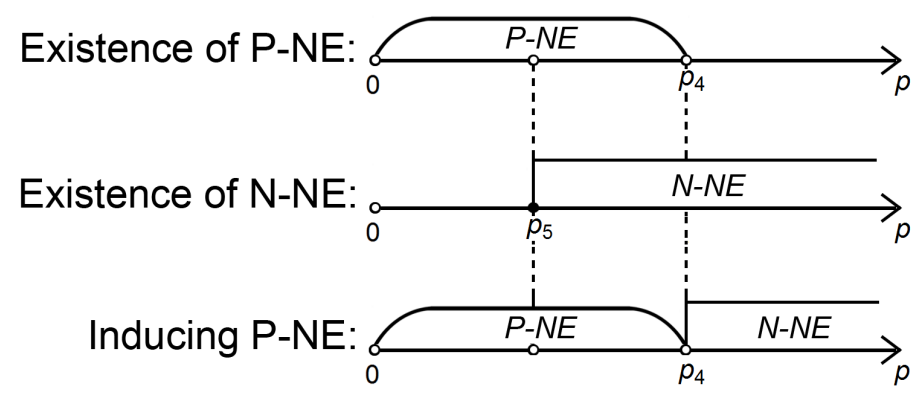

Figure 3.7: Linear cost. Optimal strategy for policy and CBT (M-S with policy)

and as the coordination motive strengthens ( $r$ increases).

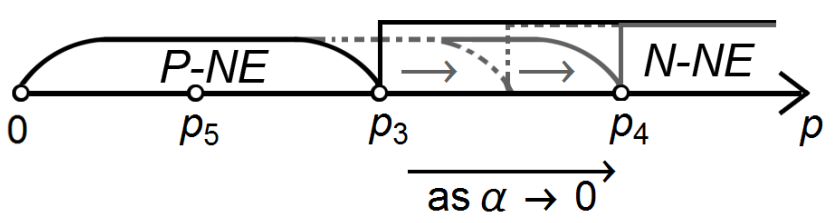

Figure 3.8: Linear cost. Disruption of a N-NE by CBT adjustment (M-S with policy)

As an aside, we consider the option of inducing a P-NE when a N-NE is optimal for some initial positive value of $\alpha$. This is possible for any $p$ such that $\left.p_{3}\right|_{0<\alpha}<$ $p<p_{4}$. Figure 3.8 demonstrates an adjustment in CBT form some positive $\alpha$ to zero. This corresponds to the widening of the price range for which a P-NE exists with the upper critical value changing from some initial $p_{3}$ to $p_{4}$. Suppose that, prior to CBT adjustment, the actual $p$ lies in the range $\left.p_{3}\right|_{0<\alpha}<p<p_{4}$, the CB fully offsets the shock $\theta$, and individual agents find it optimal not to purchase $x_{i}$. By lowering the level of CBT all the way to zero the $\mathrm{CB}$ extends the range for which private information acquisition is optimal, and this range now includes the actual $p$. This leads to disruption of the N-NE and establishment of a P-NE instead (commitment to optimal policy intervention $\rho=\rho^{*}$ ). In fact, the comparison of expected equilibrium welfare outcomes ${ }^{47} E\left[W^{N} \mid 0<\alpha\right]$ and $E\left[W^{P} \mid \alpha=0\right]$ confirms that such CBT adjustment is beneficial from the point of view of aggregate welfare.

\footnotetext{
${ }^{47}$ We compare the outcomes under the assumption of some positive $\alpha$ and $p$ such that $\left.p_{3}\right|_{0<\alpha}<$ $p<p_{4}$.
} 


\subsubsection{Efficiency of equilibria}

We are now in the position to assess the efficiency of the equilibrium outcome in a setup with direct policy intervention. We begin by looking at information acquisition. Comparing the two critical price values $\tilde{p}$ and $p_{4}$ - associated with (3.137) and (3.149) - reveals that $p_{4}<\tilde{p}$. Inefficiencies in acquisition and use of information parallel $^{48}$ those in the no-policy case. In P-NE agents put inefficiently low weight on private information $\left(\kappa_{1}<\tilde{\kappa}_{1}^{P}\right)$, and direct policy intervention is unable to remedy that. In order to compensate, the CB puts excess weight on its private signal compared to the weight put on publicly available information in the efficient benchmark $\left(\tilde{\kappa}_{2}^{P}<\left|\rho^{*}\right|\right)$. Inefficient use of information leads to agents choosing not to purchase private signals when it is collectively efficient to do so $\left(p_{4} \leq p<\tilde{p}\right)$. Agents choose inefficiently high quality of information at lower values of actual price:

$$
0<p<\frac{4(2+r-2 \sqrt{1+r})}{\gamma^{2} r^{2}}
$$

and inefficiently low (and eventually zero) quality of information at higher values of $p$ :

$$
\frac{4(2+r-2 \sqrt{1+r})}{\gamma^{2} r^{2}}<p .
$$

Efficiency outperforms the equilibrium solution for $0<p<\tilde{p}$. The welfare outcome is the same when there is no private information acquisition in either scenario (for $\tilde{p} \leq p$ ). Endogenising private information through a linear cost term prevents direct policy from achieving an efficient welfare outcome, as it does in James and Lawler (2011).

\subsubsection{Effect of policy on private information acquisition}

Finally, we look at the solutions with and without direct policy intervention to assess the effect of policy on private information acquisition. The comparison of critical price values - from (3.129) and (3.149) - creates three price ranges of interest.

From the Table 3.6, it is evident that the presence of policy broadens the set of parameter value combinations for which individual agents choose a positive $\beta$. In other words, information acquisition takes place (P-NE) at higher values of cost, at which there would be no acquisition (N-NE) in the no-policy scenario. When

\footnotetext{
${ }^{48}$ The two values of $\beta$ - in the no-policy and the with-policy cases - are equally responsive to $p$, due to the equilibrium degree of coordination being the same. Introducing policy merely leads to a parallel shift in $\beta$ as a function of $p$.
} 


\begin{tabular}{l|c|c|c} 
& $0<p<p_{1}$ & $p_{1} \leq p<p_{4}$ & $p_{4} \leq p$ \\
\hline Without policy: & $\mathrm{P}-\mathrm{NE}$ & $\mathrm{N}-\mathrm{NE}$ & $\mathrm{N}-\mathrm{NE}$ \\
With policy: & $\mathrm{P}-\mathrm{NE}$ & $\mathrm{P}-\mathrm{NE}$ & $\mathrm{N}-\mathrm{NE}$ \\
\hline$\beta$ value: & higher $\mathrm{w} /$ policy & higher $\mathrm{w} /$ policy & same \\
Welfare outcome: & superior w/policy & superior w/policy & same
\end{tabular}

Table 3.6: Linear cost. Effect of policy on private information acquisition (M-S)

actual information cost is below $p_{1}$, in the presence of policy agents have the incentive to choose a higher value of beta; when, however, price $p$ exceeds $p_{4}$, policy has no effect on information acquisition in that agents do not find it worthwhile to purchase $x_{i}$ in either case. The welfare outcome in the presence of policy (and zero $\mathrm{CBT}$ ) is superior to the no-policy scenario (with full CBT) over the price range for which information acquisition takes place in the policy case. Over the price range for which there is no information acquisition in both scenarios, welfare outcomes are the same. The effect of policy on information acquisition and welfare in P-NE constitutes Proposition 3.3.

Proposition 3.3: If agents aim to maximise a $M-S$ payoff function and information cost is linear, commitment to an optimally designed monetary policy rule in combination with optimal level of transparency with respect to the central bank's private signal achieve higher investment in private information acquisition for a wider range of unit price $p$, as well as superior welfare outcomes when such investment is positive.

\subsection{Linear Cost: James-Lawler Payoff Function}

\subsubsection{In the absence of policy}

In this Section we repeat the analysis carried out in Section 3.5 for model variations with linear information cost and J-L payoff. From (3.2), agent $i$ 's payoff function is given by:

$$
u_{i}=-\left[a_{i}-\theta+r\left(a_{i}-\bar{a}\right)\right]^{2}-\beta_{i} p .
$$

The information cost structure is linear in the quality $\beta_{i}$ of the privately purchased signal $x_{i}$. Per unit cost of information quality $p$ is given exogenously.

\subsubsection{Private information acquisition}

Given agents' optimal response function in (3.30)-(3.31) and (3.32)-(3.33), we can derive the solutions for $\beta_{i}$ and $\beta$. We take unconditional expectations of the payoff function in (3.2), substitute optimal weights into the resulting expression, and max- 
imise it with respect to $\beta_{i}$. The first-order condition is quadratic in $\beta_{i}$, thus, solving it yields two roots. One root is negative for all parameter values and, thereby, is of no interest to us, as we assume that the quality of private information is non-negative. The remaining root is positive for some parameter value combinations:

$$
\beta_{i}=\frac{(1+r)(1-\alpha \sqrt{p}) \alpha+(1+r-\alpha \sqrt{p}) \beta}{[(1+r) \alpha+\beta] \sqrt{p}} .
$$

It is apparent from (3.152) that $\beta_{i}$ is strictly increasing in the $\beta$ chosen by other agents. For a symmetric solution, we set the right-hand side in (3.152) equal to $\beta$ and solve for the latter:

$$
\beta=\frac{1+r}{\sqrt{p}}-(1+r) \alpha
$$

where $\beta$ is strictly decreasing in price $p$ and quality of public signal $\alpha$. The former relationship is due to $p$ being the marginal cost of acquiring $x_{i}$ with $\beta$ quality, and the latter has to do with $\alpha$ and $\beta$ being strategic substitutes ${ }^{49}$.

Although the M-S and J-L payoff functions are characterised by the same equilibrium degree of coordination and agents' best response coefficients (prior to substitution of endogenously determined $\beta$ ), the difference in how coordination enters the two payoff functions leads to a difference in how $r$ enters the expressions for optimal $\beta$ in (3.127) and (3.153). As a result, in the J-L case endogenously determined $\beta$ is strictly increasing $r$. The coordination parameter in the positive term of (3.153) reflects that a more accurate $x_{i}$ reduces the loss from agent $i$ 's own action's dispersion around the aggregate action. While the coordination parameter in the negative term characterises how the perceived benefit of coordination amplifies the crowding-out effect that $\alpha$ has on $\beta$. Here the net effect of an increase in $r$ on the optimal value of $\beta$ is positive.

In order to determine the critical price value below which information acquisition takes place and a symmetric P-NE exists, we identify the parameter combinations for which $\beta$ in (3.153) is strictly positive:

$$
0<p<\frac{1}{\alpha^{2}}
$$

From (3.154), we define:

$$
p_{5} \equiv \frac{1}{\alpha^{2}}
$$

When $0<p<p_{5}$, a P-NE exists and agents choose to acquire private information

\footnotetext{
${ }^{49}$ It should be noted that the coefficient on $\alpha$ in (3.153) is consistent with the general expression for endogenous precision of private information derived in Colombo et al. (2014).
} 
with quality identified in (3.153); whereas, when $p_{5} \leq p$, a N-NE exists and agents choose $\beta=0$, which is equivalent to not acquiring $x_{i}$.

\subsubsection{Central bank transparency}

We now turn to the choice faced by the $\mathrm{CB}$ - socially optimal setting of the the CBT tool $\alpha$. We substitute the best response weights from (3.32)-(3.33) into (3.43):

$$
E[W]=-(1+r)^{2} \cdot \frac{\beta}{[(1+r) \alpha+\beta]^{2}}-\frac{(1+r)^{2} \alpha}{[(1+r) \alpha+\beta]^{2}}-\beta p
$$

and then the equilibrium solution for $\beta$ in (3.153):

$$
E[W]=-\frac{\sqrt{p}-\alpha p}{1+r}-(1+r)^{2} \alpha p-(1+r)\left(\frac{1}{\sqrt{p}}-\alpha\right) p
$$

In (3.157), the three loss terms correspond to dispersion loss, volatility loss, and information cost, respectively. Dispersion loss and information cost are decreasing in $\alpha$, and volatility loss is increasing in $\alpha$. Higher quality of public information crowds out private information acquisition, resulting in a lower $\beta$, which, in turn, reduces agents' ability to forecast each others actions. As a secondary effect, a rise in $\alpha$ translates into an decrease in $\kappa_{1}$, since agents shift the weight in their response towards a now more accurate $y$ signal. As a tertiary effect, the crowding-out of $\beta$ draws agents' attention further away from $x_{i}$. As a result, the combined effect on $\kappa_{1}$ outweighs the reduction in forecasting ability, and the dispersion loss is reduced. The aforementioned effects are amplified in the dispersion loss term (which includes the $(1+r)^{2}$ multiplier) due to dispersion having an additional negative value at

the aggregate level. As far as volatility loss is concerned, an increase in the quality of public information improves the alignment between the average action and the fundamental. However, as a secondary effect, agents shift their focus towards the public signal, thereby amplifying the loss from volatility. Finally, the tertiary effect of crowding-out of $\beta$ that works through $\kappa_{2}$ further amplifies the loss. Secondary and tertiary effects outweigh the effect of improvement in accuracy of $y$ on agents' forecasting ability, thus, volatility loss is increasing in $\alpha$.

It should be noted, that, on the one hand, the social benefit of coordination weakens the tertiary effects operating through $\kappa_{1}$ and $\kappa_{2}$, as compared to the M-S case. This, in turn, dampens the effect of changes in $\alpha$ on dispersion and volatility losses. On the other hand, since dispersion loss is multiplied by $(1+r)^{2}$, the effect of changes in $\alpha$ on the combined dispersion loss term is amplified. Overall, expected equilibrium welfare is $1+2 r$ times more responsive to changes in $\alpha$, compared to 
the M-S case.

The net effect of an increase in the quality of public information on expected social welfare in a P-NE is positive, hence, it is optimal for the CB to choose full CBT $(\alpha=\gamma)$ and publish its signal with no additional noise. For convenience, we introduce:

$$
p_{6} \equiv \frac{1}{\gamma^{2}}
$$

to denote $p_{5}$ from (3.155) under full CBT. Expected equilibrium outcome in a P-NE is then given by:

$$
E\left[W^{P}\right]_{\alpha=\gamma}=p\left[(1+2 r) \gamma-\frac{2(1+r)}{\sqrt{p}}\right]
$$

where $0<p<p_{6}$.

When $p_{6}<p$ and agents do not find it worthwhile to purchase private information, a N-NE exists, the $\mathrm{CB}$ commits to full CBT and expected welfare is given by (3.46). In order to be able to assess the efficiency of equilibrium solutions derived in this Section, we first need to characterise an efficient benchmark case for the J-L payoff function with linear information cost.

\subsubsection{Efficient benchmark}

Assuming that a benevolent CB is able to instruct agents on the collectively efficient acquisition and use of information, we maximise the expected welfare in (3.43) with respect to $\kappa_{1}$ and $\kappa_{2}$ :

$$
\begin{aligned}
& \tilde{\kappa}_{1}^{P}=\frac{\beta}{\beta+(1+r)^{2} \gamma} \\
& \tilde{\kappa}_{2}^{P}=1-\tilde{\kappa}_{1}^{P},
\end{aligned}
$$

where full CBT $(\alpha=\gamma)$ is also one of the efficient benchmark assumptions. It should be noted that the coordination parameter is present in the efficient weights $\tilde{\kappa}_{1}^{P}$ and $\tilde{\kappa}_{2}^{P}$ due to coordination having a positive social value. Substituting said weights into (3.43) yields:

$$
E[W]_{\kappa_{1}=\tilde{\kappa}_{1}^{P}}=-\frac{(1+r)^{2}}{\beta+(1+r)^{2} \gamma}-\beta p .
$$

Maximising (3.162) with respect to $\beta$, we find ${ }^{50}$ :

$$
\tilde{\beta}=\frac{1+r}{\sqrt{p}}-(1+r)^{2} \gamma
$$

\footnotetext{
${ }^{50}$ It should be noted that the coefficient on $\gamma$ in (3.163) is consistent with the general expression for welfare-maximising precision of private information derived in Colombo et al. (2014).
} 
which is positive for:

$$
0<p<\frac{1}{(1+r)^{2} \gamma^{2}}
$$

Thus, we define the efficient critical price as:

$$
\tilde{p} \equiv \frac{1}{(1+r)^{2} \gamma^{2}}
$$

It is collectively efficient for private information acquisition to take place when $0<$ $p<\tilde{p}$ and for agents not to purchase private signals for $\tilde{p}<p$. Substituting the optimal value of $\beta$ from (3.163) and $\beta=0$ into (3.162), we get the purchasing and non-purchasing efficient outcomes:

$$
\begin{aligned}
& E\left[\tilde{W}^{P}\right]=(1+r)^{2} \gamma p-2(1+r) \sqrt{p} \\
& E\left[\tilde{W}^{N}\right]=-\frac{1}{\gamma},
\end{aligned}
$$

respectively.

\subsubsection{Efficiency of equilibria}

Having characterised the efficient benchmark solution, we are in the position to evaluate the equilibrium outcomes in the absence of direct policy intervention. Firstly, the critical price expressions relate to each other in the following way: $\tilde{p}<p_{6}$. This means that there is a price range for which information acquisition takes place in equilibrium, but not the efficient benchmark case, as demonstrated in Figure 3.9. The $\tilde{p}<p<p_{6}$ price range exists because agents perceive coordination to be less

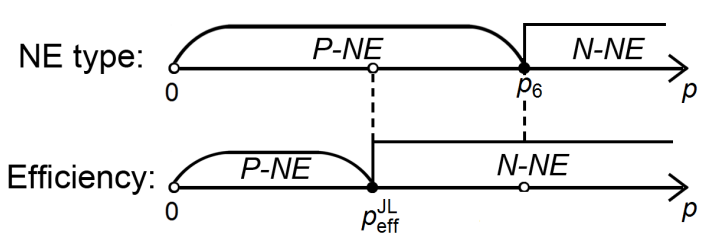

Figure 3.9: Linear cost. Equilibrium and efficient information acquisition (J-L)

beneficial than it actually is at the aggregate level. Given the same parameter values, equilibrium $\beta$ exceeds its collectively efficient counterpart:

$$
\frac{1+r}{\sqrt{p}}-(1+r)^{2} \gamma<\frac{1+r}{\sqrt{p}}-(1+r) \gamma
$$

from (3.153) and (3.163). In the efficient case, the crowding-out effect of public information quality on the collectively efficient value of $\beta$ is stronger than in equilibrium, 
as the coordination multiplier is greater in the former case: $(1+r)^{2}<(1+r)$. Thus, as price $p$ is increasing, the value of collectively efficient $\beta$ is consistently lower than equilibrium $\beta$ up until $p=\tilde{p}$, at which point private information acquisition ceases to be collectively efficient yet still takes places in equilibrium. For $p_{6} \leq p$, agents no longer purchase private signals, which corresponds to efficient information acquisition.

As far as information use is concerned, in P-NE individual agents put excess weight on their private signals. Substituting $\beta$ from (3.153) and (3.163) into (3.32) and (3.160), respectively, yields:

$$
\begin{aligned}
& \kappa_{1}^{P}=1-\gamma \sqrt{p} \\
& \tilde{\kappa}_{1}^{P}=1-(1+r) \gamma \sqrt{p}
\end{aligned}
$$

where very clearly $\tilde{\kappa}_{1}^{P}<\kappa_{1}^{P}$. It is collectively efficient for agents to put more weight on the public signal, as it is a better predictor of the average action, which is, in turn, conducive to coordination. As it is, the use of information in P-NE is not efficient. Whereas in N-NE, agents put full weight on the public signal, which is also the collectively efficient strategy.

Comparing the expected equilibrium welfare outcomes from (3.159) and (3.46) and the efficient benchmark outcomes from (3.166)-(3.167) reveals that equilibrium welfare outcome is only efficient in N-NE. In other words, collectively efficient information acquisition and use lead to the first-best welfare outcome for $p_{6}<p$.

\subsubsection{With direct policy intervention}

We now introduce direct policy intervention into the J-L payoff function and repeat the analysis. From (3.11), agent $i$ 's payoff function is given by:

$$
u_{i}=-\left[a_{i}-\theta-g+r\left(a_{i}-\bar{a}\right)\right]^{2}-\beta_{i} p
$$

where $g=\rho z$.

\subsubsection{Purchasing equilibria: private information acquisition}

Given the optimal best-response weights in (3.26)-(3.27) and (3.28)-(3.29), we maximise the expected payoff with respect to $\beta_{i}$. The relevant critical point is:

$$
\beta_{i}=\frac{(1+r)(\alpha+\beta) \sqrt{p(\gamma-\alpha \rho+\gamma \rho)^{2}}}{[(1+r) \alpha+\beta] \gamma p}-\alpha .
$$


Solving $\beta_{i}=\beta$ yields:

$$
\beta=\frac{(1+r) \sqrt{p(\gamma-\alpha \rho+\gamma \rho)^{2}}}{p \gamma}-(1+r) \alpha
$$

Parameter value combinations for which the critical point is positive are given in Table 3.7, where they are split into separate cases based on $\mathrm{CBT}^{51}$.

\begin{tabular}{l|l|l|l|l} 
Case & CBT & Policy & Cost & $x_{i}$ quality \\
\hline 1 & Zero & $\rho \neq-1$ & $0<p$ & $\beta=\frac{(1+r) \sqrt{(1+\rho)^{2}}}{\sqrt{p}}$ \\
2 & Int. & $\rho \neq-\frac{\gamma}{\gamma-\alpha}$ & $p<p_{7}$ & $\beta=\frac{(1+r) \sqrt{(\gamma-\alpha \rho+\gamma \rho)^{2}}}{\gamma \sqrt{p}}-(1+r) \alpha$ \\
3 & Full & - & $p<p_{6}$ & $\beta=\frac{1+r}{\sqrt{p}}-(1+r) \gamma$
\end{tabular}

Table 3.7: Linear cost. Conditions for a positive $\beta$ (J-L with policy)

The critical value of $p$ in Table 3.7 is defined as:

$$
p_{7} \equiv \frac{(\gamma-\alpha \rho+\gamma \rho)^{2}}{\alpha^{2} \gamma^{2}}
$$

which collapses to $p_{6}$ from (3.158) under full CBT:

$$
p_{6}=\frac{1}{\gamma^{2}}
$$

Whenever parameter value combinations lie outside the above three cases, the critical point is non-positive. It should be noted, that Case 3 in Table 3.7 is of no interest to us in this Section, as policy becomes neutral under full CBT.

\subsubsection{Purchasing equilibria: instrument adjustment and CBT}

Once equilibrium values of $\kappa_{1}, \kappa_{2}$, and $\beta$ from (3.26)-(3.27) and (3.169) are substituted into (3.41), expected equilibrium welfare expression becomes:

$$
E\left[W^{P}\right]_{\beta=\beta^{*}}=2 \cdot \frac{(1+r)\left[\alpha \gamma p-\sqrt{(\gamma-\alpha \rho+\gamma \rho)^{2} p}\right]}{\gamma}-\frac{\alpha \gamma^{2} p+(\gamma-\alpha) \rho^{2}}{\gamma^{2}}
$$

In (3.171), the first term is the sum of dispersion loss and information cost which are equal to each other, meaning that the remaining term is the loss from volatility.

${ }^{51}$ Zero, Int., and Full correspond to $\alpha=0,0<\alpha<\gamma$, and $\alpha=\gamma$, respectively. 
Expression (3.171) is maximised for ${ }^{52}$ :

$$
\rho^{*}=-(1+r) \gamma \sqrt{p}
$$

The optimal value of the policy parameter $\rho$ is strictly increasing in the coordination parameter, quality of CB's private signal, and unit cost of private information quality.

Having derived the optimal policy setting, we can narrow down the parameter value combinations for which a P-NE exists. Given $\rho=\rho^{*}$, optimal $\beta$ is positive for parameter combinations given in Table 3.8.

\begin{tabular}{l|l|l|l|l} 
Case & CBT & Policy & Cost & $x_{i}$ quality \\
\hline 1 & Zero & $\rho=\rho^{*}$ & $0<p<p_{9}$ & $\beta=\frac{1+r}{\sqrt{p}}-(1+r)^{2} \gamma$ \\
2 & Int. & $\rho=\rho^{*}$ & $0<p<p_{8}$ & $\beta=\frac{1+r}{\sqrt{p}}-(1+r)[\gamma+r(\gamma-\alpha)]$
\end{tabular}

Table 3.8: Linear cost. Conditions for a positive $\beta$, given $\rho=\rho^{*}$ (J-L with policy)

The critical value of $p$ in Table 3.8 is defined as:

$$
p_{8} \equiv \frac{1}{[\gamma+r(\gamma-\alpha)]^{2}}
$$

which collapses to:

$$
p_{9} \equiv \frac{1}{(1+r)^{2} \gamma^{2}},
$$

under zero CBT. When $0<\alpha<\gamma$, optimal value of $\beta$ is:

$$
\left.\beta\right|_{\rho=\rho^{*}} ^{\operatorname{Int}}=\frac{1+r}{\sqrt{p}}-(1+r)[\gamma+r(\gamma-\alpha)]
$$

obtained by setting $\rho=\rho^{*}$ in (3.169). Under zero CBT, expression (3.175) becomes:

$$
\left.\beta\right|_{\rho=\rho^{*}} ^{\text {Zero }}=\frac{1+r}{\sqrt{p}}-(1+r)^{2} \gamma
$$

Given updated conditions on $p$ and $\rho=\rho^{*}$, the term under the square root in the expected welfare expression in (3.171) is positive, thus, the expression can be

\footnotetext{
${ }^{52}$ Maximisation yields two roots. The positive root leads to a lower expected welfare outcome, thus, we focus on the negative root.
} 
further simplified:

$$
E\left[W^{P}\right]_{\beta=\beta^{*}, \rho=\rho^{*}}=-2 \cdot(1+r)\{1-[\gamma+r(\gamma-\alpha)]\} \sqrt{p}-\left[\alpha+(1+r)^{2}(\gamma-\alpha)\right] p .
$$

Again, in (3.177), the first term is the sum of dispersion loss and information cost, where the two are equal to each other. Expected welfare in (3.177) is strictly decreasing in $\alpha$, which implies that minimum level of CBT is optimal: $\alpha=0$. Expected equilibrium welfare outcome in $\mathrm{P}-\mathrm{NE}$ is then given by:

$$
E\left[W^{P}\right]_{\alpha=0}=(1+r)^{2} \gamma p-2(1+r) \sqrt{p} .
$$

Commitment to zero CBT narrows down the conditions consistent with the existence of P-NE to Case 1 in Table 3.8.

As far as use of information in P-NE is concerned, agent $i$ 's best response weights are:

$$
\begin{aligned}
& \kappa_{1}^{P}=1+\rho^{*}=1-(1+r) \gamma \sqrt{p} \\
& \kappa_{2}^{P}=0,
\end{aligned}
$$

where $0<\kappa_{1}^{P}<1$ and $-1<\rho^{*}<0$. By committing to zero CBT, the CB effectively chooses to reserve its private information exclusively for instrument adjustment. This implies that a weight equal to $\left|\rho^{*}\right|$ is being put on signal $z$ by the $\mathrm{CB}$ and weight $1-\left|\rho^{*}\right|$ is being put on privately acquired signals.

\subsubsection{Non-purchasing equilibria}

Having characterised the P-NE for the J-L case with direct policy intervention, we look at characterising N-NE. As a first step, we identify the conditions for which optimally chosen $\beta$ in (3.169) is non-positive. Parameter combinations for which $\beta$ is non-positive or, in other words, conditions for N-NE are given in Table 3.9.

\begin{tabular}{l|l|l|l} 
Case & CBT & Policy & Cost \\
\hline 1 & Zero & $\rho=-1$ & $0<p$ \\
$2 \mathrm{a}$ & Int. & $\rho=-\frac{\gamma}{\gamma-\alpha}$ & $0<p$ \\
$2 \mathrm{~b}$ & Int. & $\rho \neq-\frac{\gamma}{\gamma-\alpha}$ & $p_{7} \leq p$ \\
3 & Full & - & $p_{6} \leq p$
\end{tabular}

Table 3.9: Linear cost. Conditions for a non-positive $\beta$ (J-L with policy) 
Table 3.9 refers to:

$$
\begin{aligned}
& p_{6}=\frac{1}{\gamma^{2}} \\
& p_{7}=\frac{(\gamma-\alpha \rho+\gamma \rho)^{2}}{\alpha^{2} \gamma^{2}}
\end{aligned}
$$

from (3.170) and (3.158). As before, Case 3 is of no relevance in this Section due to policy neutrality under full CBT.

In the absence of private information acquisition, the $\mathrm{CB}$ finds it optimal to commit to a one-to-one policy rule and expected welfare outcome is given by (3.49). Thus, the conditions for existence of a N-NE in Table 3.9 are narrowed down to two Cases and presented in Table 3.10.

\begin{tabular}{l|l|l|l} 
Case & CBT & Policy & Cost \\
\hline 1 & Zero & $\rho=-1$ & $0<p$ \\
$2 \mathrm{~b}$ & Int. & $\rho=-1$ & $p_{6} \leq p$
\end{tabular}

Table 3.10: Linear cost. Conditions for a non-positive $\beta$, given $\rho=-1$ (J-L with policy)

From (3.158), the critical price value in Table 3.10 is $p_{6}=\frac{1}{\gamma^{2}}$. In both Cases 1 and $2 \mathrm{~b}$ agents are passive. When $p_{6}<p$, the policymaker can choose any value of $\alpha$ below $\gamma$ without it having any effect on the expected welfare outcome (and without precluding the existence of a N-NE).

\subsubsection{Inducing a purchasing equilibrium}

Comparison of conditions for existence of P-NE and N-NE reveals that there exist parameter value combinations for which both types of NE occur. Namely, when the unit cost of private information quality lies in the range $0<p<p_{9}$, both $\mathrm{P}-\mathrm{NE}$ and N-NE may exist. The former is characterised by $\rho=\rho^{*}$, the latter is characterised by $\rho=-1$, and both are characterised by zero $\mathrm{CBT}^{53}$. This creates the scope for the policymaker to be able to induce a particular type of NE by choosing one policy rule over another.

In a P-NE, the information set of the economy includes CB's private signal $z$ as well as private signals $x_{i}$. Since accuracies $\gamma$ and $\beta$, that are associated with observed signals, are positive, inclusion of $x_{i}$ in the information set increases information accuracy. Higher information accuracy increases agents' ability to forecast the shock,

\footnotetext{
${ }^{53}$ When $p_{6} \leq p$, the N-NE can be characterised by any level of CBT, in which case there is no overlap.
} 
CB's private signal, and other agents' signals. This benefit is counteracted by the cost of purchasing $x_{i}$ as well as a higher dispersion loss due to lower commonality of information. In a N-NE, information accuracy is lower, as economy's information set no longer includes private signals. On the other hand, social welfare is not affected by information cost or action dispersion, since agents follow a passive strategy. The question arises whether the net benefit of information acquisition in P-NE outweighs the benefits of perfect coordination and zero information cost in N-NE.

In order to identify which type of NE is preferable over the price range $0<p<p_{9}$, we compare the expected welfare outcomes from (3.178) and (3.49). Private information acquisition leads to a superior welfare outcome, as $E\left[W^{P}\right]$ strictly exceeds $E\left[W^{N}\right]$ over the price range in question. In other words, the social value of higher information accuracy is enough for a P-NE to be socially preferable. Thus, the optimal strategy for the $\mathrm{CB}$ is to commit to $\rho=\rho^{*}$ thereby inducing a P-NE for $0<p<p_{9}$ and follow a one-to-one policy rule for $p_{9} \leq p$, as demonstrated in Figure 3.10 .

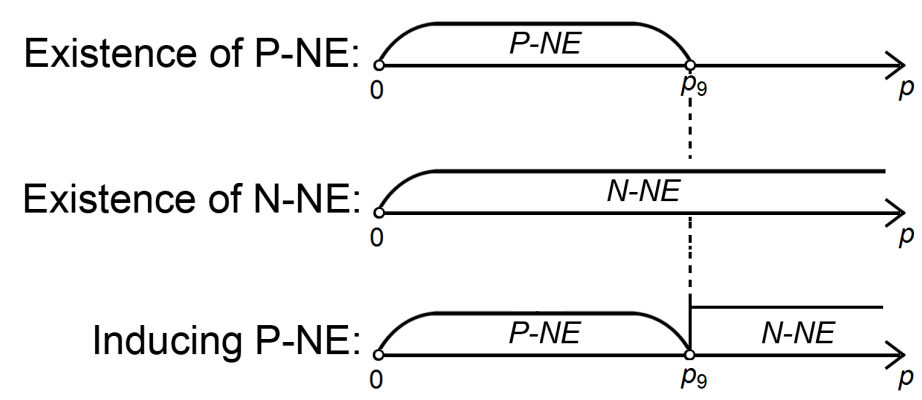

Figure 3.10: Linear cost. Optimal strategy for policy and CBT (J-L with policy)

\subsubsection{Efficiency of equilibria}

Having identified CB's optimal strategy and corresponding equilibrium outcomes, we can assess the efficiency of the latter using the benchmark case derived in the no-policy section. We compare expressions for private information accuracy from (3.163) and (3.176), critical price from (3.165) and (3.174), expected P-NE welfare 
from (3.166) and (3.178), expected N-NE welfare from (3.167) and (3.49):

$$
\begin{aligned}
\tilde{\beta} & =\left.\beta\right|_{\rho=\rho^{*}} ^{\text {Zero }}=\frac{1+r}{\sqrt{p}}-(1+r)^{2} \gamma \\
\tilde{p} & =p_{9}=\frac{1}{(1+r)^{2} \gamma^{2}} \\
E\left[\tilde{W}^{P}\right] & =E\left[W^{P}\right]_{\alpha=0, \rho=\rho^{*}}=(1+r)^{2} \gamma p-2(1+r) \sqrt{p} \\
E\left[\tilde{W}^{N}\right] & =E\left[W^{N}\right]_{\rho=-1}=-\frac{1}{\gamma} .
\end{aligned}
$$

We find that direct policy intervention allows the CB to achieve efficient information acquisition as well as first-best expected welfare outcomes.

Efficiency of the equilibrium solution is in line with the result in James and Lawler (2011). More specifically, the combination of an optimally designed policy rule and zero CBT encourage efficient use of privately acquired information by individual agents, while allowing the $\mathrm{CB}$ to use its own private signal efficiently. Comparing expressions from (3.160)-(3.161) $)^{54},(3.172)$, and (3.179)-(3.180):

$$
\begin{aligned}
& \tilde{\kappa}_{1}^{P}=\kappa_{1}^{P}=1-(1+r) \gamma \sqrt{p} \\
& \tilde{\kappa}_{2}^{P}=\rho^{*}=-(1+r) \gamma \sqrt{p} \\
& \kappa_{2}^{P}=0
\end{aligned}
$$

demonstrates that equilibrium use of information is efficient, yet the agency between the benchmark and the equilibrium cases differs. In the former, individual agents put weight $\tilde{\kappa}_{2}^{P}$ on the public signal when choosing their action, whereas in the latter, the $\mathrm{CB}$ puts a weight $\rho^{*}$ on its private signal when adjusting the policy instrument.

In contrast to the M-S case, introduction of endogenous private information acquisition into the model with a re-formulated J-L payoff function does not preclude the success of policy in obtaining the social optimum.

\subsubsection{Effect of policy on private information acquisition}

The above conclusion about efficiency of equilibria in the presence of policy reveals that an optimally designed rule achieves collectively efficient information acquisition for all parameter values, in both P-NE and N-NE. Meanwhile, equilibrium strategies in the absence of direct policy intervention lead to inefficient acquisition as well as use of information in P-NE, which, in turn, contribute to suboptimal welfare outcomes. Table 3.11 summarises the comparison between the without-policy and

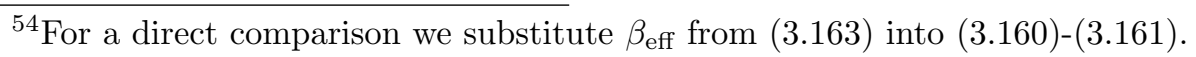


with-policy cases.

\begin{tabular}{l|c|c|c} 
& $0<p<p_{9}$ & $p_{9} \leq p<p_{6}$ & $p_{6} \leq p$ \\
\hline Without policy: & $\mathrm{P}-\mathrm{NE}$ & $\mathrm{P}-\mathrm{NE}$ & $\mathrm{N}-\mathrm{NE}$ \\
With policy: & $\mathrm{P}-\mathrm{NE}$ & $\mathrm{N}-\mathrm{NE}$ & $\mathrm{N}-\mathrm{NE}$ \\
\hline$\beta$ value: & lower $\mathrm{w} /$ policy & lower w/policy & same \\
Welfare outcome: & superior w/policy & superior w/policy & same
\end{tabular}

Table 3.11: Linear cost. Effect of policy on private information acquisition (J-L)

Table 3.11 presents three price ranges that emerge from comparing critical price values from (3.158) and (3.174).

In the absence of policy, individual agents have an inefficiently high incentive to acquire private information. This leads to the existence of a price range $\left(p_{9} \leq p<p_{6}\right)$ for which equilibrium value of $\beta$ is positive (P-NE), yet the collectively efficient $\tilde{\beta}$ is zero (N-NE). This also means that over the price range, for which information acquisition takes place in both equilibrium and efficient benchmark $\left(0<p<p_{9}\right)$, the equilibrium value of $\beta$ is consistently higher in value than its efficient counterpart: $0<\tilde{\beta}<\beta^{*}$.

The presence of policy narrows down the set of parameter value combinations for which individual agents choose a positive $\beta$. By adjusting agents' incentive to acquire private signals, optimally designed policy brings information acquisition in line with the efficient benchmark case. It should be noted that this is only true for $0<p<p_{6}$. When $p_{6} \leq p$, direct policy intervention and CBT on its own achieve the same outcome rendering policy intervention superfluous. This leads us to Proposition 3.4.

Proposition 3.4: If agents aim to maximise a J-L payoff function and information cost is linear, commitment to an optimally designed monetary policy rule in combination with optimal level of transparency with respect to the central bank's private signal achieve efficient use of information, efficient information acquisition, as well as the first-best welfare outcome for all values of $p$.

The effect of direct policy intervention on P-NE is the reverse of that in the M-S case. This is not surprising, given that the J-L payoff function is formulated so as to have the opposite implications for coordination and value of public information. Namely, coordination is perceived to be of inefficiently high value in the M-S case, whereas it is inefficiently undervalued in the J-L case. Through mechanisms discussed in detail above, this translated into an inefficiently low and an inefficiently 
high incentives to purchase $x_{i}$, respectively. Thus, in order to attempt to counteract these inefficiencies, the CB has to implement instrument adjustment that has opposing effects on information acquisition in the M-S and the J-L cases.

\subsection{Concluding remarks}

In the present Chapter we looked at how direct policy intervention and central bank transparency perform in a coordination game with private information acquisition. We solve our model eight times for every combination of the following pairs of alternative assumptions: (i) individual agents are given the opportunity to pay either a fixed fee for a private signal of given quality or a linear cost for a signal of their preferred quality, (ii) individual agents aim to maximise either the re-formulated Morris-Shin or James-Lawler payoff function, and (iii) the central bank is either able to adjust solely the quality of the public signal or also able to impact outcomes directly through instrument adjustment. In each case we evaluate optimal policy design by comparing it to a benchmark case with efficient use and acquisition of information.

In all scenarios we distinguish between purchasing and non-purchasing Nash equilibria, however, the precise definitions of the NE types differ for fixed-cost and linear-cost scenarios. Under the fixed-cost assumption, a P-NE is defined by all agents purchasing a private signal of given quality and incurring a flat fee, whereas a N-NE is defined by all agents opting to not purchase private information. The term information acquisition stands for whether or not an individual agent chooses to purchase a private signal at a given price, and information use stands for the weights attached to each observed signal in individual agent's best response.

In this Chapter, we solve and analyse the fixed-cost scenarios first. In the absence of direct policy intervention, information use is inefficient in the P-NE due to the misalignment between the perceived benefit of coordination at the individual and aggregate levels in equilibrium. This result merely replicates the well-established argument in the literature for the re-formulated payoff functions. Inefficiency in the use of information does not arise in the N-NE. We demonstrate that, in P-NE, inefficient use of information leads to inefficient acquisition of information for some price values. At the same time, we also show that efficient use of information on its own is insufficient to guarantee efficient acquisition. In the M-S case, the CB is able to induce efficient information acquisition for some price values by committing to a particular CBT regime. In the J-L case, the $\mathrm{CB}$ is unable to use CBT to induce a particular type of NE, since full CBT is invariably optimal. 
In the presence of direct policy intervention, the $\mathrm{CB}$ is able to use commitment to an optimally designed rule and appropriately selected CBT regime to induce a socially preferable type of NE. This is the case for both payoff functions. Partially offsetting the shock leads to a P-NE, whereas offsetting the shock fully leads to a N-NE. In line with the result obtained by James and Lawler (2011), commitment to zero CBT allows the CB to induce efficient use of private information by the private sector, while making the most of its own private information to offset the shock. The CB is able to achieve efficient use and acquisition of information, as well as the first-best expected welfare outcome. The $\mathrm{CB}$ is unable to achieve this result when equipped with CBT as its only policy tool.

Under the linear-cost assumption, a P-NE is defined by all agents purchasing a private signal of the same non-zero quality and incurring a linear fee, whereas a $\mathrm{N}-\mathrm{NE}$ is defined by all agents opting to purchase a private signal with zero quality (which is equivalent to not purchasing a private signal at all). In this case, the term information acquisition stands for quality of private information an individual agent chooses to purchase at a given per-unit (of precision) price, and the meaning of information use remains the same as in the fixed-cost case.

In the absence of direct policy intervention, we replicate the results presented in Colombo and Femminis (2008) for the re-formulated M-S payoff. Namely, we show that the introduction of linear cost and endogenous quality of private information prevents the standard Morris-Shin result from arising, i.e. increasing the quality of public information is always beneficial for social welfare under the assumption of private information acquisition. In other words, full CBT is optimal for all parameter values, which is also true for the J-L payoff. Inefficient use of information in $\mathrm{P}-\mathrm{NE}$ leads to inefficient information acquisition for some price values. In the M-S case, agents purchase private signals of quality that is inefficiently high at low prices and inefficiently low at intermediate prices. As a result, N-NE arises when it is not collectively efficient. In the J-L case, agents purchase private signals of inefficiently high quality in P-NE, which leads to P-NE arising when it is not collectively efficient. Expected welfare outcomes are first-best and use as well as acquisition of information are efficient in N-NE for some price values.

In the presence of direct policy intervention, it is optimal for the CB to induce a $\mathrm{P}-\mathrm{NE}$ for some price values, and the $\mathrm{CB}$ is able to do so by committing to an optimally designed rule in combination with zero CBT. In the M-S case, optimal instrument adjustment and zero CBT fails to achieve efficiency and first-best welfare 
outcome for some price values, however, policy intervention mitigates inefficiencies that arise in its absence. In the J-L case, optimal policy design enables the CB to achieve efficiency and first-best outcome for all price values. We fail to pinpoint the reason why the presence of linear cost prevents policy from completely eliminating inefficiency in the M-S case, however, it is likely to do with the re-formulation of the payoff function.

In our linear-cost analysis, an important point of contact with literature is the substitutability between the quality of public and private information, which is implied by the obtained expressions for endogenous precision of private information in equilibrium (without policy) and the efficient benchmark for both payoff functions. The relationships identified in this Chapter are consistent with the general expressions for equilibrium and collectively efficient precisions presented in Colombo et al. (2014). 


\section{Chapter 4}

\section{Market Signal Value and Costly Information}

\subsection{Introduction}

As discussed in Section 1.5.3, market prices can act as a valuable source of information for a monetary authority. By observing aggregate market activity, a policymaker is potentially able to construct a more accurate picture of the current state of the economy and adjust its policy accordingly. In other words, in the eyes of a central bank, market aggregates have signal value. This value, however, is sensitive to the fact that market participants are aware that their actions are being monitored to inform monetary policy. In theory, such awareness has a negative consequence for the signal value of market prices. Namely, the more weight the central bank puts on market prices when determining its instrument adjustment, the less informative those prices become. Following Morris and Shin (2018), we refer to this negative relationship as the reflection problem.

In the present Chapter we look at the signal value of market prices and the reflection problem in the context of a one-shot simultaneous-move game. More specifically, we focus on the three-way interaction between the signal value of market aggregates, monetary policy, and costly information acquisition. For this purpose, we heavily rely on the model of monetary policy and a financial market presented in Morris and Shin (2018) and extend it by using the approach to modelling private information acquisition discussed in Colombo and Femminis (2008). The latter has already been applied in the previous Chapter, whereas the former is introduced in Section 4.2.1 below.

In contrast to scenarios presented in the previous Chapters, we move away from 
considering direct monetary policy intervention that aims to maximise the aggregate of individual payoffs across agents by mitigating an exogenous shock. Instead, we follow Morris and Shin (2018) in assuming that the central bank publicly commits to a rule that reacts to the market aggregate, and the aim of the rule is to neutralise the shock with no direct concern over how policy affects market outcomes. The reflection problem arises because we assume that market participants are interested in aligning their actions with the bank's instrument adjustment, while being aware that the instrument adjustment is based on the aggregate of their actions. Individual agents seeking to align their actions with the policy instrument (and indirectly with the average action) results in the deterioration of the signal value of the aggregate action. The aggregate action conveys less information about the shock than it would do were market participants solely interested in aligning their actions with the shock. This reflection mechanism becomes the key factor in the central bank's decision about the parameter values of its rule.

The signal value of the market aggregate depends on how well it reflects the diverse private information about economic fundamentals available to individual agents. By assuming that private information is itself endogenous, we add yet another dimension to strategic market interactions. Now individual decisions to invest in private information acquisition need to be reconciled with the desire to align actions with monetary policy, which, in turn, depends on the quality of private information conveyed through the market aggregate. As a results, the central bank needs to consider how its decision about rule parameters affects the individual agents' action strategies as well as information acquisition strategies.

The present Chapter is divided into two parts based on the assumptions we make about information acquisition. In the first part, we follow Colombo and Femminis (2008) in assuming that individual agents choose how much to invest in acquiring private information, and the cost is a linear term in quality of the purchased private signals. We also relate our results to those in Colombo et al. (2014), in which the authors analyse private information acquisition in a class of models with strategic complementarity or substitutability, heterogeneous private signals, and a common public signal ${ }^{1}$. Colombo and Femminis and Colombo et al. find that the quality of agents' private information is inversely related to the quality of the public signal. Thus, when private information acquisition is introduced into the Morris and Shin (2018) model to construct the scenario explored here, the relationship between the quality of the public signal and the signal value of the market aggregate becomes

\footnotetext{
${ }^{1}$ The more general framework in Colombo et al. (2014) includes the Colombo and Femminis (2008) model as a special case.
} 
more complex than the result reported by Morris and Shin.

In the second part of this Chapter, we consider costly acquisition of public information. As opposed to the linear-cost modelling approach used by Colombo and Femminis (2008), we use a fixed-cost method already employed in the previous Chapter $^{2}$. Namely, we assume that market participants choose whether of not to acquire a common signal for a flat fee, while the quality of their private information remains exogenous. The signal value of the market aggregate that contains information from both heterogeneous private information and the public information is diluted compared to the market signal that is based on the private information alone. Thus, acquisition of public information by the market participants is undesirable in the eyes of the policymaker, yet the value of public information plays a key role in strategic market interactions. The nature of how public information enters the reflection problem determines the considerations the central bank needs to take into account when designing an optimal policy rule.

In what follows, we first introduce the model in Section 4.2.1 in the context of private information acquisition. We then discuss a benchmark variation of the model without the reflection problem in Section 4.2.2. We present the discussion of purchasing equilibria, including monetary policy considerations, in Section 4.2.3 and the discussion of non-purchasing equilibria in Section 4.2.4. We begin the second part of this Chapter by introducing the scenario with public information acquisition in Section 4.3.1. We proceed by solving for purchasing and non-purchasing equilibria in Sections 4.3.2 and 4.3.3, respectively. Finally, we discuss optimal policy design in relation to the public information acquisition in Section 4.3.4.

\subsection{Private information acquisition}

\subsubsection{Model}

The state variable $\theta$, referred to also as the shock, is drawn from an improper prior and represents economic fundamentals. The market consists of an infinite number of agents arranged over the interval $i \in[0,1]$. Individual agent $i$ observes two signals about $\theta$ : a private signal $x_{i}=\theta+\varepsilon_{i}$ and a public signal $y=\theta+\eta$, where $\varepsilon_{i} \sim N\left(0, \frac{1}{\beta_{i}}\right)$ and $\eta \sim N\left(0, \frac{1}{\alpha}\right)$. Prior to observing $x_{i}$, agent $i$ chooses the precision $\beta_{i}$ which costs price $p$ per unit of precision. Thus, the individual information cost

\footnotetext{
${ }^{2}$ In Chapter 3, the fixed-cost approach was applied in the context of the acquisition of heterogeneous private information, whereas here we look at acquisition of a common signal about the fundamental.
} 
is given by $p \cdot \beta_{i}$. Choosing $\beta_{i}=0$ is equivalent to not acquiring private information. A symmetric equilibrium in which all agents acquire a private signal such that $0<\beta_{i}=\beta$ is referred to as a purchasing Nash equilibrium or $P$-NE, while an equilibrium in which no agents find it optimal to acquire a private signal is a non-purchasing Nash equilibrium or $N$-NE.

The central bank (CB) observes it own private signal about $\theta: z=\theta+\phi$, where $\phi \sim N\left(0, \frac{1}{\gamma}\right)$. Following Morris and Shin (2018), we assume that the CB does not observe the public signal $y^{3}$. Instead, the $\mathrm{CB}$ observes the average action across the market and interprets it as a market-based signal about $\theta$. The market average action is defined as $\bar{a} \equiv \int_{0}^{1} a_{i} d i$, where $a_{i}$ is agent $i$ 's action.

The CB's objective is to minimise its loss given by:

$$
L=(g-\theta)^{2},
$$

where $g$ is CB's monetary policy instrument adjustment. We further assume that the $\mathrm{CB}$ is able to credibly commit to a forward guidance rule ${ }^{4}$ given by:

$$
g=\tau \bar{a}+(1-\tau) z
$$

where $0<\tau<1$ is the weight ${ }^{5} \mathrm{CB}$ places on the market-based signal ${ }^{6}$. It should be noted that we follow Morris and Shin (2018) in referring to the monetary policy rule as the (Odyssean) forward guidance rule in the sense of an announcement that publicly commits a policymaker to a particular course of action (Campbell et al., 2012). On the one hand, the policy rule in (4.2) is formulated contrary to the policy

\footnotetext{
${ }^{3}$ Morris and Shin refer to $y$ as "conventional wisdom or fashionable market narrative that takes hold among market participants...in a fleeting way compared to the time scale of the monetary process" (Morris and Shin, 2018, p. 2). Arguably, it is difficult to imagine information that all agents are able to observe with certainty, yet the CB is either unable to observe or chooses to ignore, which makes this assumption the weakest point of the Morris and Shin model when it comes to interpretation and policy implications. From an algebraic standpoint, the assumption that $y$ is not observable prevents the CB from perfectly inferring $\theta$ from the market-based signal, making it crucial for modelling purposes. As far as the use of similar assumptions in related literature is concerned, Lubik et al. (2020) solve a New Keynesian model in which households have access to information without error, while the $\mathrm{CB}$ only observes noisy measurements of inflation and real GDP.

${ }^{4}$ In the present Chapter we set aside considerations of discretionary policy. The primary reason for not devoting attention to the non-commitment case lies in its intractability once the model is extended to include information acquisition.

${ }^{5}$ Weight $\tau$ in our model is the equivalent of $\lambda$ in Morris and Shin (2018).

${ }^{6}$ In this Chapter we refer to $\tau$ as being chosen by the CB. However, the value for the rule parameter can be selected by a designer of the policy regime that is a separate entity from the $\mathrm{CB}$. The agency for the choice of $\tau$ does not matter as long as the CB and the policymaker are assumed to have the same monetary policy objective.
} 
recommendation given by Bernanke and Woodford (1997) in that it relies on a variable that is, as will be demonstrated, sensitive to the expectations of the public $(\bar{a})$. On the other hand, Bernanke and Woodford propose using supplementary information to inform policy decisions, which is captured by the presence of $z$ in the rule. Individual agent $i$ aims to maximise a payoff function given by:

$$
u_{i}=-\omega\left(a_{i}-g\right)^{2}-(1-\omega)\left(a_{i}-\theta\right)^{2}-p \beta_{i}
$$

where $\omega \in(0,1)$. Thus, agent $i$ incurs a loss from imperfectly matching its action $a_{i}$ to the CB's instrument adjustment and the state variable, as well as an information cost. It should be noted that CB's objective function in (4.1) is chosen not be equal to the aggregate of individual agents' payoffs, which is in contrast to the original Morris and Shin (2002) framework and variants thereof. Here for simplicity we follow Morris and Shin (2018) in assuming that the alignment of agents' actions with the state variable is not intrinsic to CB's objective, and, thus, the CB's sole aim is to neutralise the shock $\theta$.

It should be noted, that agent $i$ 's payoff in (4.3) does not include an explicit coordination term, which sets this model apart from Morris-Shin style coordination games $^{7}$. Instead the coordination motive arises endogenously through agents' desire to match CB's instrument adjustment which, in turn, relies on the average action. Following Angeletos and Pavan (2007b), the equilibrium degree of coordination ${ }^{8}$ (or EDoC) charactering this model is equal to $\omega \tau$. Thus, the strength of the implicit coordination motive depends on how much importance market participants give to matching CB's instrument adjustment as well as how much the CB relies on the average action as a source of information. Angeletos and Pavan characterise models by comparing the EDoC to a collectively efficient degree of coordination (or CEDoC). Deriving the latter demands the construction of an efficient benchmark model which, in turn, requires additional assumptions to be made about social welfare. One option is to define social welfare as the aggregate of individual payoffs: $W=\int_{0}^{1} u_{i} d i$, which implies that there is a social benefit to matching the instrument adjustment. Another option is to follow Morris and Shin (2002) in assuming that there is no social benefit to coordination and only the distance between agents' actions and $\theta$ matters: $W=-\int_{0}^{1}\left(a_{i}-\theta\right)^{2} d i-p \beta$. In both cases, if the social welfare expression differs from CB's objective function, there is a need to provide a rationale for why the CB does not act in a strictly benevolent manner. Here, we set these possibilities

\footnotetext{
${ }^{7}$ Such as, but not limited to Morris and Shin $(2002,2005)$, Angeletos and Pavan (2007a,b), and James and Lawler (2011).

${ }^{8}$ Found by differentiating agent $i$ 's optimal action with respect to the average action, or, formally, $u_{a_{i} \bar{a}}^{i} /\left|u_{a_{i} a_{i}}^{i}\right|$.
} 
aside.

The model in this Chapter is a sequential non-cooperative game with a simultaneousmove subgame. The sequence of events in the model are as follows:

1. the $\mathrm{CB}$ announces parameter $\tau$ and commits to its rule;

2. individual agents simultaneously and privately decide on the quality of their private signals $\left(\beta_{i}\right)$;

3. the shock $\theta$ is realised, agents observe signals $x_{i}$ and $y$, form expectations of $g$ and $\theta$, and take their actions $\left(a_{i}\right)$;

4. the CB observes the average action $\bar{a}$ as well as its private signal $z$, forms its expectation of $\theta$, and adjusts instrument $g$;

5. finally, agents' payoffs and the CB's loss are determined.

By announcing its policy rule in the first step, the CB acts as a Stackleberg leader, and the announced rule parameter enters agents' decision-making in the second step. Due to the game having multiple steps, the solution method will be that of backward induction.

Before we proceed to solve the model, we first look at a special-case scenario in which there is no endogenously arising coordination. We accomplish this by assuming that the CB's rule no longer relies on the market signal. As a result, we are able to characterise private information acquisition in the absence of coordination. The special case serves as a useful benchmark at a later stage of our analysis.

\subsubsection{Benchmark for private information acquisition $(\tau=0)$}

Consider a special-case setup in which the CB's rule is simply $g=z$ and the CB does not observe the market signal. Agent $i$ chooses action $a_{i}$ so as to maximise payoff in (4.3). Thus, agent $i$ 's optimal strategy is given by:

$$
a_{i}=\omega E_{i}[g]+(1-\omega) E_{i}[\theta]
$$

where $E_{i}[\cdot]$ stands for agent $i$ 's expectations conditional on observing $x_{i}$ and $y$. Given that $g=z$, we can substitute $E_{i}[g]=E_{i}[z]$, and $E_{i}[z]=E_{i}[\theta]$ holds because agents have no further information about CB's private signal. Due to $E_{i}[g]=E_{i}[\theta]$, agent $i$ 's two objectives - to align action $a_{i}$ with the CB's instrument adjustment and to align $a_{i}$ with the fundamental - coincide. Since the weights on the two 
objectives add up to unity $(\omega+(1-\omega)=1)$, agent $i$ 's optimal strategy is to set the action equal to conditional expectation of $\theta$ :

$$
a_{i}=E_{i}\left[\theta \mid x_{i}, y\right]=\frac{\alpha y+\beta_{i} x_{i}}{\alpha+\beta_{i}} .
$$

In fact, the first of the two equalities in (4.4) implies an EDoC equal to zero, since the average action is absent from the agent $i$ optimal strategy. For the linear equilibrium solution of the form:

$$
a_{i}=\kappa_{i} x_{i}+\left(1-\kappa_{i}\right) y
$$

the equilibrium weight on the private signal $\kappa_{i}$ is a Bayesian weight:

$$
\hat{\kappa}_{i}=\frac{\beta_{i}}{\alpha+\beta_{i}} .
$$

As seen from (4.6), the optimal strategy for agent $i$ is to weight the two observed signals according to their relative precisions. The weight $\hat{\kappa}_{i}$ does not include any variables that are under the control of the $\mathrm{CB}$, meaning that there is no mechanism of reflection present.

Taking expectations of the payoff in (4.3), while assuming $g=z$, yields:

$$
E\left[u_{i}\right]=-\frac{\kappa_{i}^{2}}{\beta_{i}}-\frac{\left(1-\kappa_{i}\right)^{2}}{\alpha}-\frac{\omega}{\gamma}-p \beta_{i} .
$$

Substituting the Bayesian weight from (4.6) into (4.7) gives:

$$
E\left[u_{i}\right]_{\kappa_{i}=\hat{\kappa}_{i}}=-\frac{1}{\alpha+\beta_{i}}-\frac{\omega}{\gamma}-p \beta_{i}
$$

Although the expected payoff above includes a loss term in $\omega$, this parameter is measuring the relative importance of matching CB's instrument adjustment and does not actually affect private information acquisition or its use. Expression (4.8) is maximised for ${ }^{9}$ :

$$
\beta_{i}=\hat{\beta}=\frac{1}{\sqrt{p}}-\alpha .
$$

In line with the result reported by Colombo and Femminis (2008) and Colombo et al. (2014), demand for private information quality is strictly decreasing in both the cost of information and the quality of publicly available information ${ }^{10}$. The authors refer to the latter relationship as the crowding-out effect of public information. The

\footnotetext{
${ }^{9}$ The other root is negative for all parameter values and, thus, irrelevant.

${ }^{10}$ In fact, the expression for optimal $\beta$ found in Colombo and Femminis (2008) is reduced to (4.9) in the limit, as the strength of coordination motive in their model approaches zero.
} 
strength of the crowding-out effect of public information on private information ${ }^{11}$ is amplified in the presence of a coordination motive and mitigated in the presence of an action-misalignment motive. As noted above, neither motive is present in this benchmark scenario, as evidenced by the EDoC being equal to zero. This results in one-to-one substitutability between quality of public and private information, or, in other words, an increase in $\alpha$ by one unit of precision leads to a decrease in $\hat{\beta}$ by the same amount.

A P-NE exists when equilibrium $\hat{\beta}$ in (4.9) is positive. From this restriction we get:

$$
0<p<\frac{1}{\alpha^{2}}
$$

from which we can define:

$$
\hat{p} \equiv \frac{1}{\alpha^{2}}
$$

The condition in (4.10) is the necessary and sufficient condition for P-NE. Consequently, when the actual information price is equal to or exceeds $\hat{p}$, a N-NE exists, in which no information acquisition takes place and agents set their action equal to the public signal $y$.

Having characterised equilibrium use and acquisition of private information as well as conditions for NE existence in the absence of strategic complementarities, we proceed to solve the model for $0<\tau<1$. In other words, we return to the assumption that the $\mathrm{CB}$ commits to a rule that responds to both its private information and the market signal, which creates indirect strategic complementarity in agents' actions.

\subsubsection{Solving for purchasing equilibria (P-NE)}

We begin by looking at P-NE. We first discuss agents' use of information and the quality of the market signal. The relevant subsection is largely devoted to reproducing results obtained in Morris and Shin (2018) with a change in notation and additional comments. We then turn to private information acquisition and how it affects the market signal. Finally, we set out the CB's minimisation problem as well as present a numerical example.

\footnotetext{
${ }^{11}$ Also referred to as "substitutability between the two sources of information" (Colombo et al., 2014, p.1447)
} 


\subsubsection{Agent $i$ 's best response and CB's information quality}

In general terms, agent $i$ 's payoff-maximising strategy is the same as in the specialcase scenario above:

$$
a_{i}=\omega E_{i}[g]+(1-\omega) E_{i}[\theta]
$$

which, after substituting for $g$, becomes:

$$
a_{i}=\omega \tau E_{i}[\bar{a}]+(1-\omega \tau) E_{i}[\theta]
$$

As before, we assume that agent $i$ 's best response can also be expressed as a linear combination of observed signals, from (4.5):

$$
a_{i}=\kappa_{i} x_{i}+\left(1-\kappa_{i}\right) y
$$

where $0<\kappa_{i}<1$. From agent $i$ 's point of view, all other market participants face a symmetric optimisation problem and, thus, their best response actions are characterised by the same value of the weight $\kappa_{j}=\kappa$, where $i \neq j$. The average action is then given by:

$$
\bar{a}=\kappa \theta+(1-\kappa) y,
$$

which, of course, is also the market signal observed by the CB.

Given the above definition of average action in (4.13), we can further characterise CB's information set. Firstly, we can express CB's forecast of $\theta$ as weighted average of the two signals $\bar{a}$ and $z$ :

$$
E[\theta \mid \bar{a}, z]=\frac{\alpha \bar{a}+\gamma(1-\kappa)^{2} z}{\alpha+\gamma(1-\kappa)^{2}}
$$

Secondly, we can derive the expression for variance of CB's forecast errors:

$$
\operatorname{Var}[\theta-E[\theta \mid \bar{a}, z]]=E\left[(\theta-E[\theta \mid \bar{a}, z])^{2}\right]-(E[\theta-E[\theta \mid \bar{a}, z]])^{2}
$$

where the second term on the right-hand side is equal to zero, and substitute for $E[\theta \mid \bar{a}, z]$ from (4.14). The inverse of the variance of forecast errors is the combined accuracy of CB's signals of $\theta$, which is given by:

$$
\operatorname{Accuracy}^{\mathrm{CB}}=\frac{\alpha}{(1-\kappa)^{2}}+\gamma
$$

From (4.13), unconditional expectation of the average action is a linear combination of $\theta$ and $y$. In the limit, as $\kappa \rightarrow 1$, individual agents completely ignore the public signal, expected average action collapses to just $\theta$ and becomes a perfect signal 
of the state variable and accuracy of CB's information tends to an infinitely large value. If, on the other hand, individuals were to completely ignore their private signals $(\kappa=0)$, expected average action would collapse to just the public signal and accuracy of CB's information would be equal to the sum of accuracies of the two remaining signals: $\alpha+\gamma$. Expression (4.15) reflects that, for any $0<\kappa<1$, accuracy of CB's information lies somewhere between those two extremes, with any increase in $\kappa$ taking us closer to the CB's perfect information scenario. Any increase in the quality of signals $y$ and $z$, naturally, improves the accuracy of CB's information set.

Substituting $\bar{a}$ from (4.13) into best-response in (4.12) along with agent $i$ 's conditional expectation of the state variable $E_{i}\left[\theta \mid x_{i}, y\right]$ yields an expression that is linear in $x_{i}$ and $y$. Comparing coefficients on the resulting expression and the alternative specification of agent $i$ 's best response in (4.5) yields:

$$
\kappa_{i}=\frac{[1-(1-\kappa) \omega \tau] \beta_{i}}{\alpha+\beta_{i}} .
$$

Setting $\beta_{i}=\beta$ and $\kappa_{i}=\kappa$ in the above expression and solving the resulting equation for $\kappa$, we get:

$$
\kappa^{*}=\frac{\beta(1-\omega \tau)}{\alpha+\beta(1-\omega \tau)} .
$$

The presence of the term $\omega \tau$ (which is the EDoC in this model) in (4.16) reflects the endogenously arising strategic complementarity. It is apparent from (4.16), that agent $i$ weights the private signal disproportionately to its relative precision, in contrast to the special-case benchmark, in which $\hat{\kappa}$ in (4.6) is a Bayesian weight. In fact, $\kappa^{*}$ (for $0<\tau<1$ ) is strictly smaller than $\hat{\kappa}$ (for $\tau=0$ ) meaning that agent $i$ places less emphasis on private information compared to the benchmark. This is in line with the results reported by Morris and Shin (2005) and Amador and Weill (2010), who find that agents put less weight on their private signals in the presence of public information ${ }^{12}$. Substituting (4.16) into the above solution for $\kappa_{i}$, yields:

$$
\kappa_{i}=\frac{(\alpha+\beta) \beta_{i}(1-\omega \tau)}{\left(\alpha+\beta_{i}\right)[\alpha+(1-\omega \tau) \beta]}
$$

Both expressions (4.16) and (4.17) are strictly decreasing in the policy weight $\tau$, which constitutes the reflection problem. Namely, the more weight the CB puts on the average action as a signal of $\theta$, the less weight market participants put on their private signals, which in turn leads to a decrease in the accuracy of CB's information about the state variable, as indicated by (4.15).

\footnotetext{
${ }^{12}$ In contrast to Morris and Shin (2005) and Amador and Weill (2010), here the release of the public signal $y$ is outside of CB's control.
} 


\subsubsection{Information acquisition}

Following the method of backward induction, we move on to study agent $i$ 's choice of private signal quality $\beta_{i}$. We do so by maximising agent $i$ 's expected payoff with respect to $\beta_{i}$. First, we take unconditional expectations of (4.3) after substituting for $g$ :

$$
E\left[u_{i}\right]=-\frac{\kappa_{i}^{2}}{\beta_{i}}-\frac{\left(1-\kappa_{i}\right)^{2}-\omega \tau(1-\kappa)\left[2-\tau(1-\kappa)-2 \kappa_{i}\right]}{\alpha}-\frac{\omega(1-\tau)^{2}}{\gamma}-p \beta_{i} .
$$

We substitute for $\kappa_{i}$ and $\kappa$ from (4.17) and (4.16) in the expected payoff and maximise the resulting expression with respect to $\beta_{i}$. We find two roots, one of which is always negative and, thus, not relevant to our discussion of P-NE. The other root is positive for some parameter value combinations:

$$
\beta_{i}=\frac{(\alpha+\beta)(1-\omega \tau)}{[\alpha+\beta(1-\omega \tau)] \sqrt{p}}-\alpha
$$

In a symmetric equilibrium, the solution for $\beta_{i}$ is symmetric across all agents, meaning that $\beta_{i}=\beta$ for all $i$. Setting the right-hand side of (4.19) equal to $\beta$ gives a quadratic equation, solving which for $\beta$ yields:

$$
\beta^{*}=\frac{1}{\sqrt{p}}-\frac{\alpha}{1-\omega \tau}
$$

Expression in (4.20) is one of the two roots with the other root being negative for all parameter values and, thus, inadmissible. From (4.20), it is apparent that $\beta^{*}$ is strictly decreasing in $p, \alpha, \omega$, and $\tau$. The above solution for $\beta$ is analogous to that in Colombo and Femminis (2008) with the only difference being that here the endogenously determined degree of coordination $\omega \tau$ takes on the role played by the exogenously given coordination parameter in the Colombo and Femminis model. The two distinct payoff functions - that assumed by Colombo and Femminis (2008) and that adopted here - imply a difference in the nature of coordination. Namely, the payoff function in Colombo and Femminis (2008) is a variation of the MorrisShin payoff (Morris and Shin, 2002) which features direct strategic complementarity in the form of the beauty-contest term. Here, the individual agent's payoff gives rise to actions which indirectly (through the CB's instrument adjustment) exhibit a strategic complementarity. Despite the difference in the payoff functions and the nature of coordination, the parallels in agents' optimal strategies ${ }^{13}$ appear to be sufficient to produce an analogous result with respect to information acquisition.

\footnotetext{
${ }^{13}$ Expression in (4.12) is directly comparable to expression (2) in Colombo and Femminis (2008). The two are equivalent for $\omega \tau=r$, where $r$ is the EDoC in Colombo and Femminis notation.
} 
The above result is a clear expansion on the discussion in Colombo et al. (2014) who analyse private information acquisition in a class of models with direct strategic complementarity or substitutability. Colombo et al. demonstrate that the precision of public information enters the expression for optimal precision of private signals with a coefficient equal to:

$$
-\frac{1}{1-\mathrm{DoC}}
$$

where DoC refers to equilibrium or collectively efficient degree of coordination, depending on the scenario considered. In the present case, we identified the equilibrium (endogenous) degree of coordination to be equal to $\omega \tau$, and, thus, the absolute value of the coefficient on $\alpha$ in (4.20) is greater than unity. This means that any change in the exogenous $\alpha$ has a more than one-to-one effect on the value of $\beta^{*}$. This is in contrast to our benchmark case, in which equilibrium private information acquisition is characterised by expression (4.9) and changes in $\alpha$ have a one-to-one effect on $\hat{\beta}$. This is, of course, due to the fact that the benchmark scenario assumes $\tau=0$ thereby eliminating strategic complementarity that arises endogenously for $0<\tau<1$ and reducing the EDoC to zero. In other words, the presence of indirect strategic complementarities amplifies the effect that $\alpha$ has on $\beta^{*}$, compared to the benchmark case.

Colombo et al. (2014) explain that the precisions of public and private signals $-\alpha$ and $\beta$, respectively - act as strategic substitutes, thus, free public information has a crowding out effect on agents' investment into acquiring private information. In our model, when $0<\tau<1$, closer alignment with the CB's instrument and the average actions (through the CB's rule) has a positive effect on agents' expected payoffs. The desire to align one's own action with the actions of others brings about coordination. Due to being observed by all individual agents, the public signal is no longer just a source of information about $\theta$, but it also contributes to alignment of actions through aiding coordination. Any increase in the quality of public information facilitates coordination, thereby benefiting every individual agent through a corresponding increase in the expected payoff. With better coordination, agents give less importance to their heterogeneous private signals. This crowding-out effect is amplified through the perceived importance of coordination, which is captured by the EDoC $(\omega \tau)$. This explains why expression (4.20) is strictly decreasing in $\alpha, \omega$, and $\tau$.

Morris and Shin (2018) find that an increase in the exogenous $\beta$ leads to the CB putting more weight on the market-based signal (a higher value of equilibrium 
$\tau)$. The assumption of endogeneity of $\beta$ in the present setting creates an additional consideration for the $\mathrm{CB}$, namely, how the choice of $\tau$ affects the CB's objective through the induced change in equilibrium $\beta^{*}$. Algebraically this is demonstrated by the presence of $\tau$ in the expression for equilibrium $\beta^{*}$ in (4.20). This link between $\beta^{*}$ and $\tau$ implies that a change in exogenous variables $p, \alpha$, or $\omega$ affects agent $i$ 's choice of $\beta$ directly as well as through CB's adjustment in $\tau$ that the CB deems appropriate in response to said change. As a result, the total effect of a change in $p, \alpha$, or $\omega$ on the CB's choice of $\tau$ and the equilibrium $\beta^{*}$ is less straightforward.

A P-NE exists when the equilibrium solution for $\beta$ in (4.20) is strictly positive. Hence, the condition for existence of $\mathrm{P}-\mathrm{NE}$ is $0<p<p_{1}$, where:

$$
p_{1} \equiv \frac{(1-\omega \tau)^{2}}{\alpha^{2}}
$$

which is strictly positive for all parameter values of $\omega, \alpha$, and $\tau \in(0,1)$. The latter fact implies that there always exists a non-empty set of sufficiently low prices $p$ for which investment in private information acquisition is positive, and this is true for all $0<\tau<1$. In P-NE, agent $i$ places the following weight on their private signal:

$$
\left.\kappa^{*}\right|_{\beta=\beta^{*}}=1-\frac{\alpha \sqrt{p}}{1-\omega \tau},
$$

from (4.16) and (4.20). Any change in $\alpha, \omega$, or $\tau$ has a primary and a secondary effect on the value of the $\left.\kappa^{*}\right|_{\beta=\beta^{*}}$ weight. Primary effects are associated with the expression for $\kappa^{*}$ prior to substitution of $\beta=\beta^{*}$, which is given in (4.16). An increase in $\alpha$ has a negative effect on $\kappa^{*}$ as it changes the relative precision of the two observed signals $-x_{i}$ and $y$. Higher relative precision of $y$ leads to less weight being put on $x_{i}$. An increase in $\omega$ or $\tau$ affects the strength of endogenous coordination, which increases the perceived importance of the public signal $y$. Secondary effects work through the endogenous $\beta^{*}$. As discussed above, an increase in $p, \alpha, \omega$, or $\tau$ leads to a decrease in the equilibrium value of $\beta$ which, in turn, leads to a smaller $\kappa$ weight as the relative precision of $x_{i}$ is reduced. On the one hand, lower quality of $x_{i}$ reduces agents $i$ 's ability to predict $\theta$ as well as CB's signal $z$ and the $\theta$-component of $\bar{a}$. On the other hand, lower $\kappa$ reduces the importance of the $\theta$-component of $\bar{a}$ thereby improving agent $i$ 's ability to align its action with $\bar{a}$. A more detailed discussion of how agent $i$ 's ability to forecast $g$ is affected by changes in $\beta, p, \alpha, \omega$, or $\tau$ is presented in Appendix D.1.

Endogenising $\beta$ changes the way $\tau$ enters agent $i$ 's optimal strategy. In the absence of private information acquisition, equilibrium $\kappa$ - given in (4.16) - lies 
in the interval $\left(\frac{\beta(1-\omega)}{\alpha+\beta(1-\omega)}, \frac{\beta}{\alpha+\beta}\right)$, depending on the value of $\tau$ chosen by the CB. Equilibrium $\kappa$ is strictly positive for $0<\tau<1$. In the presence of private information acquisition, agent $i$ 's optimal $\kappa$ - given in (4.23) - lies in the interval $\left(1-\frac{\alpha \sqrt{p}}{1-\omega}, 1-\alpha \sqrt{p}\right)$, for $0<\tau<1$. It is apparent that, for $0<p$, there exist parameter value combinations for which optimal $\kappa$ is non-positive for either some or all values of $\tau$. Individual agents place a strictly positive weight on their private signals when $0<p<p_{1}$. Of course, this is also the necessary and sufficient condition for the existence of a P-NE. In other words, the existence of a P-NE guarantees that the equilibrium $\kappa$-weight is positive, and vice versa.

\subsubsection{Agent $i$ 's expected equilibrium payoff in $\mathrm{P}-\mathrm{NE}$}

In order to take a closer look at agent $i$ 's expected equilibrium payoff it is helpful to decompose the expected payoff into meaningful terms. For this purpose, we use the approach already employed in the previous Chapter. Namely, following Angeletos and Pavan (2007b), we identify a dispersion loss and a volatility loss within the expression for agent $i$ 's expected payoff. The decomposition procedure is presented in more detail in Appendix D.2.

Agent $i$ 's expected payoff is given in (4.18). In a symmetric equilibrium, $\beta_{i}=\beta$ and $\kappa_{i}=\kappa$, thus, expression in (4.18) becomes:

$$
E\left[u_{i}\right]=-\frac{\kappa^{2}}{\beta}-\frac{\left(1-2 \omega \tau+\omega \tau^{2}\right)(1-\kappa)^{2}}{\alpha}-\frac{\omega(1-\tau)^{2}}{\gamma}-p \beta,
$$

where the terms correspond to:

$E\left[u_{i}\right]=-$ Dispersion $-\left(1-2 \omega \tau+\omega \tau^{2}\right) \cdot$ Volatility $-\omega(1-\tau)^{2} \cdot$ Variance of $\phi$-Information cost.

The term in the variance of $\phi$ (or variance of $z$ as a forecast of $\theta$ ) arises because $z$ is a noisy signal about $\theta$. Even if agent $i$ was able to match $\theta$ perfectly, agent $i$ 's ability to match $z$ would be affected by the presence of noise $\phi$. As far as the coefficient on volatility loss is concerned, it should be noted that:

$$
(1-\omega)<\left(1-2 \omega \tau+\omega \tau^{2}\right)<1
$$

which captures two observations about the "endogenous" weight on alignment between the average action and $\theta$ that arises after substituting CB's rule into the payoff. On the one hand, the endogenous weight on volatility $\left(1-2 \omega \tau+\omega \tau^{2}\right)$, as seen in $(4.24)$, is greater than the weight $(1-\omega)$ that agents place on matching $\theta$ prior to considering the rule in (4.2). As noted above, this is due to agent $i$ not being 
able to observe $z$ and, thus, the aim of matching the $z$-component of the CB's rule coinciding with the aim of matching $\theta$. This creates an additional volatility term with a coefficient $\omega(1-\tau)^{2}$. On the other hand, the coefficient on volatility loss is strictly below unity. A coefficient equal to unity corresponds to the case in which agents only care about matching their actions with $\theta$. The latter would be the case if either the aim of matching CB's instrument was absent from the payoff $(\omega=0)$ or the CB's rule did not respond to the average action as a market signal $(\tau=0)$. For $0<\omega<1$ and $0<\tau<1$, agents are able to better match ${ }^{14}$ the part of the CB's rule that responds to the market signal $\bar{a}$ than $\theta$ or $z$, thereby, reducing the volatility loss.

The effects that a change in parameter values has on agent $i$ 's expected payoff can be conveniently split into primary, secondary, and tertiary. Primary effects are direct and can be characterised by looking at (4.24):

$$
E\left[u_{i}\right]=-\frac{\kappa^{2}}{\beta}-\frac{\left(1-2 \omega \tau+\omega \tau^{2}\right)(1-\kappa)^{2}}{\alpha}-\frac{\omega(1-\tau)^{2}}{\gamma}-p \beta .
$$

The first three terms are increasing in the quality of signals. Higher $\alpha$ reduces volatility loss, higher $\beta$ reduces dispersion loss (but increases information cost), and higher $\gamma$ reduces variance of $\phi$. As $\omega$ increases, the explicit aim of matching the state variable becomes less important, while the endogenously induced importance of matching CB's private signal $z$ grows. This reduces volatility loss (as the total effect on the $1-2 \omega \tau+\omega \tau^{2}$ coefficient is negative) and increases the loss associated with the variance of $\phi$. An increase in $\tau$ shifts the weight from matching the uncertain $z$-component of the CB's rule to matching the market signal, which agents are able to better match on average. This reduces both volatility loss and the loss from the variance of $\phi$. An increase in equilibrium $\kappa$ increases dispersion loss and reduces volatility loss, as agents put more weight on idiosyncratic private information.

Secondary effect work through equilibrium $\kappa$. Substituting $\kappa$ from (4.16) into (4.24) yields:

$$
E\left[u_{i}\right]_{\kappa=\kappa^{*}}=-\frac{\beta(1-\omega \tau)^{2}}{[\alpha+\beta(1-\omega \tau)]^{2}}-\frac{\left(1-2 \omega \tau+\omega \tau^{2}\right) \alpha}{[\alpha+\beta(1-\omega \tau)]^{2}}-\frac{\omega(1-\tau)^{2}}{\gamma}-p \beta,
$$

where the third and fourth terms are unaffected by the substitution. Equilibrium $\kappa$ is decreasing in $\omega$ and $\tau$ (due to increased endogenous coordination) and $\alpha$ (due to agent $i$ placing more weight on a more accurate public signal), and is increasing in $\beta$ (due to agent $i$ shifting the response weight to a more accurate private signal).

\footnotetext{
${ }^{14}$ The accuracy of agent $i$ 's forecast of $\theta$ is $\alpha+\beta$, whereas the accuracy of agent $i$ 's forecast of $\bar{a}$ is $\frac{(\alpha+\beta)^{2}}{\alpha \kappa^{2}}$. The latter strictly exceeds the former.
} 
Hence, changes in $\omega, \tau, \alpha$, and $\beta$ have a secondary effect on dispersion and volatility losses through equilibrium $\kappa$.

When both primary and secondary effects are taken into account, the effect of a change in $\beta$ on dispersion loss becomes ambiguous, since a change in $\beta$ creates two effects working in opposite directions. On the one hand, an increase in $\beta$ reduces dispersion, as private information becomes more precise (primary effect). On the other hand, an increase in $\beta$ leads to agents placing more weight on their private information thereby increasing the weight $\kappa^{2}$ on the dispersion component of the expected payoff (secondary effect). When both effects are taken into account, dispersion loss is decreasing in $\beta$ for $\alpha<\beta(1-\omega \tau)$. This condition involves the relative accuracy of public information as well as the degree of coordination. Volatility loss is unambiguously decreasing in $\beta$ through $\kappa$, as agents place less weight on the imperfect public signal.

Tertiary effect works through private information acquisition. Substituting equilibrium $\beta^{*}$ from (4.20) into (4.25) yields:

$$
\begin{aligned}
E\left[u_{i}\right]_{\kappa=\kappa^{*}, \beta=\beta^{*}}= & -\frac{(1-\alpha \sqrt{p}-\omega \tau) \sqrt{p}}{(1-\omega \tau)}-\frac{\left(1-2 \omega \tau+\omega \tau^{2}\right) \alpha p}{(1-\omega \tau)^{2}}- \\
& -\frac{\omega(1-\tau)^{2}}{\gamma}-\left(\sqrt{p}-\frac{\alpha p}{1-\omega \tau}\right)
\end{aligned}
$$

where the third term is unaffected by the substitution. Equilibrium precision of private information in (4.20) is decreasing in per-unit information price $p$, quality of public information $\alpha$ (due to the crowding-out effect of public information), as well as parameters $\omega$ and $\tau$ (due to increased EDoC). Any change in $p, \alpha, \omega$, or $\tau$ affects endogenously determined $\beta^{*}$ thereby also affecting dispersion and volatility losses through the tertiary effect. When all three effects are considered, dispersion loss is strictly decreasing in $\alpha, \omega$, and $\tau$ and decreasing in $p$ for $p_{1} / 4<p<p_{1}{ }^{15}$. Volatility loss is increasing in $\alpha, \tau$, and $p$; it is decreasing in $\omega$ for $1<\omega(2-\tau)$ and increasing in $\omega$ for $\omega(2-\tau)<1$ (which is true for $0<\omega \leq \frac{1}{2}$ regardless of the value of $\tau$ ). Higher values of $\tau$ increase the range of $\omega$-values for which volatility is increasing in $\omega$ (as $\omega(2-\tau)$ falls) by strengthening the secondary and tertiary effects that work through the EDoC.

When primary, secondary, and tertiary effects on all terms in (4.26) are considered, agent $i$ 's expected payoff is strictly increasing in signal quality $\alpha$ and $\gamma$.

$$
{ }^{15} \text { From }(4.22), p_{1}=\frac{(1-\omega \tau)^{2}}{\alpha^{2}} .
$$


Expected payoff is decreasing in $\omega$ when:

$$
0<\gamma<\frac{(1-\tau)^{2}(1-\omega \tau)^{3}}{\alpha \tau\left(2-\tau-\omega \tau^{2}\right) p}
$$

and increasing in $\omega$ otherwise. Expected payoff is decreasing in $p$ when:

$$
0<p<\frac{(1-\omega \tau)^{4}}{\alpha^{2}\left(1-\omega \tau^{2}\right)^{2}}
$$

and increasing in $p$ otherwise:

$$
\frac{(1-\omega \tau)^{4}}{\alpha^{2}\left(1-\omega \tau^{2}\right)^{2}}<p<p_{1}
$$

It should be noted that the above analysis is incomplete without knowing the value of $\tau$ that the CB chooses to commit to. Having obtained a solution for $\tau$ expressed in terms of model parameters and substituting it in (4.26) would introduce an additional - quaternary - effect of changes in $\alpha, \gamma, \omega$, and $p$ on the expected payoff.

Agent $i$ 's expected payoff, as expressed by (4.26), is strictly increasing in $\tau$. An increase in $\tau$ reduces dispersion loss, variance of $\phi$ loss, and information cost, yet increases volatility loss. The latter effect is dominated by the reduction in the other three loss terms. If $\tau$ was chosen to maximise a representative agent's expected payoff, it would be optimal for the CB to set $\tau$ equal to its maximum possible value and commit to $\tau=1$, or $g=\bar{a}$. If that were the case, individual agents would be able to better match the CB's instrument adjustment and would not suffer a variance of $\phi$ loss, as $z$ would no longer be part of the CB's rule. Agent $i$ 's expected payoff would then be:

$$
E\left[u_{i}\right]_{\kappa=\kappa^{*}, \beta=\beta^{*}, \tau=1}=-\frac{(2-\alpha \sqrt{p}-2 \omega) \sqrt{p}}{(1-\omega)} .
$$

However, in following the original Morris-Shin model, we assume that the CB chooses $\tau$ so as to minimise its loss function which is different from agents' payoffs. Thus, we now turn to CB's decision-making.

\subsubsection{Implications for policy design}

The presence of the parameter $\tau$ in the condition for existence of a P-NE, means that the CB needs to take into account how its choice of $\tau$ affects said condition. In 
order to look more closely at these considerations, we first decompose the $0<p<p_{1}$ condition to produce ${ }^{16}$ :

$$
\left\{\begin{array}{l}
\frac{(1-\omega)^{2}}{\alpha^{2}}<p<\frac{1}{\alpha^{2}} \\
0<\tau<\frac{1-\alpha \sqrt{p}}{\omega},
\end{array}\right.
$$

where $\frac{(1-\omega)^{2}}{\alpha^{2}}$ and $\frac{1}{\alpha^{2}}$ are $p_{1}$ from $(4.22)$ evaluated at $\tau=1$ and $\tau=0$, respectively. The significance of inequalities in (4.31) lies in their implications for policy design. Namely, that, under certain circumstances, the presence of private information acquisition can place a restriction on the range of $\tau$-values the CB can choose from. Given that exogenous parameter values satisfy the first part of (4.31), the CB's choice is restricted to the second part of (4.31) if the CB wants to maintain a P-NE. Alternatively, inequalities in (4.31) can be seen not as a restriction, but a guide to how a $\mathrm{CB}$ can use commitment to a policy rule so as to induce a preferred type of equilibrium - P-NE or N-NE. Specifically, if exogenous parameter values satisfy the first part of (4.31), choosing $0<\tau<\frac{1-\alpha \sqrt{p}}{\omega}$ induces a P-NE, whereas choosing $\frac{1-\alpha \sqrt{p}}{\omega} \leq \tau<1$ induces a N-NE. Whether or not inducing a certain type of $\mathrm{NE}$ is a dominant strategy depends on which NE is associated with the lowest expected loss for the CB. Steering the market to a NE with a particular level of investment in private information might be optimal due to factors not accounted for in this model. For instance, if the social welfare function differs from the CB's loss function, the $\mathrm{CB}$ might have the option of committing to a rule that is optimal from the point of view of the former, but not the latter. However, commitment to a rule that does not minimise CB's expected loss raises the question of credibility of such a rule. We will return to this question once we characterise the N-NE in more detail.

We next look at how endogeneity of private information acquisition affects the accuracy of CB's information. For this purpose, we essentially compare two scenarios which differ in the timing of the CB's decision about $\tau$ relative to the timing of the endogenous determination of $\beta^{*}$ : (i) first scenario follows the timeline set out in Section 4.2.1, and (ii) the second scenario assumes that the determination of $\beta^{*}$ takes place prior to determination of $\tau$, as step 0 relative to the timeline in Section 4.2.1. Scenario (i) is the model discussed in this Chapter, whereas scenario (ii) is the original model discussed in Morris and Shin (2018) with the additional assumption that the exogenously given $\beta$ in the original case is equal to $\beta^{*}$ obtained as an

\footnotetext{
${ }^{16}$ For $0<p<p_{1}$ to hold, either inequalities in (4.31) need to be satisfied or $0<p \leq \frac{(1-\omega)^{2}}{\alpha^{2}}$. The latter ensures $0<p<p_{1}$ regardless of the value of $\tau$.
} 
endogenous solution when $0<p<p_{1}$. For scenario (i) we substitute equilibrium value of $\left.\kappa\right|_{\beta=\beta^{*}}$ from (4.23) into (4.15):

$$
\operatorname{Accuracy}_{\kappa=\kappa^{*}, \beta=\beta^{*}}^{\mathrm{CB}}=\frac{(1-\omega \tau)^{2}}{\alpha p}+\gamma \text {. }
$$

It is clear from (4.32) that the more responsive the policy rule is to the observed $\bar{a}$ (the higher $\tau$ is) the less accurate the CB's information becomes.

In order to evaluate the effect of endogeneity of $\beta$, we compare (4.32) to an implied expression for accuracy from the original model discussed in Morris and Shin (2018). When $\beta$ is given exogenously, accuracy is obtained by substituting $\kappa$ from (4.16) into (4.15):

$$
\operatorname{Accuracy}_{\kappa=\kappa^{*}}^{\mathrm{CB}}=\frac{[\alpha+\beta(1-\omega \tau)]^{2}}{\alpha}+\gamma
$$

We proceed by differentiating the expressions for accuracy - (4.32) and (4.33) with respect to $\tau$ and, in order to allow comparison, we evaluate the derivative for scenario (ii) for $\beta=\beta^{*}$ :

$$
\begin{aligned}
& \frac{\partial \operatorname{Accuracy}_{\kappa=\kappa^{*}, \beta=\beta^{*}}^{\mathrm{CB}}}{\partial \tau}=-\frac{2(1-\omega \tau) \omega}{\alpha p} \\
& \left.\frac{\partial \text { Accuracy }_{\kappa=\kappa^{*}}^{\mathrm{CB}}}{\partial \tau}\right|_{\beta=\beta^{*}}=-\frac{2(1-\omega \tau) \omega}{\alpha p}+\frac{2 \alpha \omega}{\sqrt{p}}
\end{aligned}
$$

CB's information accuracy decreases in $\tau$ in both cases (both derivatives are strictly negative for $0<p<p_{1}$ ), however, the absolute value of the derivative is smaller in the exogenous- $\beta$ case ${ }^{17}$. This implies, that information acquisition exacerbates the reflection problem. In the original case (scenario (ii)), an increase in $\tau$ strengthens the endogenous coordination motive, discouraging agents from putting weight on their private signals, as the public signal is a better predictor of the average action and, thus, a better predictor of the instrument adjustment $g$. In the case with private information acquisition (scenario (i)) there is an exacerbating secondary effect that works through the optimal choice of $\beta$ : strengthening of the endogenous coordination motive discourages agents from investing in purchasing private information. Lower quality of private information further reduces $\kappa$ and, consequently, CB's information accuracy. In other words, the reflection problem is less pronounced when the forward guidance rule can be adopted after agents' private-signal precision has been fixed at

\footnotetext{
${ }^{17}$ This result is also reflected in the slopes of the equilibrium $\kappa$ expressions with respect to $\tau$. When the exogenous- $\beta$ case slope of (4.16) with respect to $\tau$ is evaluated at $\beta=\beta^{*}$ from (4.20), it is strictly smaller, in absolute terms, than the slope of (4.23) with respect to $\tau$. Thus, equilibrium $\kappa$ is more reactive to CB's setting of $\tau$ in the endogenous- $\beta$ case.
} 
$\beta_{i}=\beta=\beta^{*}$ than if the rule is already known to agents when choosing optimal $\beta_{i}$. This advantage becomes less pronounced as information costs rise $(p \uparrow)$.

\subsubsection{CB's minimisation problem and numerical examples}

Continuing with the backward induction method, we now look at the minimisation problem facing the $\mathrm{CB}$, given agents' best response and optimal information acquisition. The CB aims to minimise the loss as expressed by the unconditional expectations of the loss function in (4.1) and given the forward guidance rule in (4.2) and the average action in (4.13):

$$
E[L]=\tau^{2}(1-\kappa)^{2} \cdot \frac{1}{\alpha}+(1-\tau)^{2} \cdot \frac{1}{\gamma} .
$$

Given that agent $i$ 's best response is characterised by (4.16), expected loss in (4.36) becomes:

$$
E[L]_{\kappa=\kappa^{*}}=\frac{\alpha \tau^{2}}{[\alpha+\beta(1-\omega \tau)]^{2}}+(1-\tau)^{2} \cdot \frac{1}{\gamma} .
$$

Furthermore, we substitute the equilibrium value of $\beta$ from (4.20) in the above expression and we get:

$$
E[L]_{\beta=\beta^{*}}=\frac{\alpha \tau^{2} p}{(1-\omega \tau)^{2}}+(1-\tau)^{2} \cdot \frac{1}{\gamma}
$$

According to our derivations, solutions for $\tau$ that minimise the expected loss in (4.38), are not tractable. There exists a unique interior solution ${ }^{18}$, and let it be denoted $\tau^{P}$, then agent $i$ 's equilibrium choices are characterised by $\left.\kappa^{P} \equiv \kappa^{*}\right|_{\beta=\beta^{*}, \tau=\tau^{P}}$ and $\left.\beta^{P} \equiv \beta^{*}\right|_{\tau=\tau^{P}}$. Having defined relevant equilibrium values, we are able to state Proposition 4.1.

Proposition 4.1: Given agents make a payoff-maximising decision about private

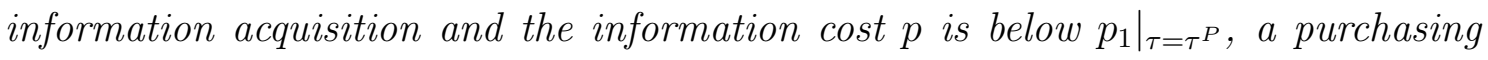
equilibrium exists and it is optimal for the central bank to commit to $\tau=\tau^{P}$.

In a $\tau \kappa$-space, the solution to the model is given by the point of tangency $\left(\tau^{P}, \kappa^{P}\right)$ where the $\kappa^{*}$-function, that represents agent $i$ 's optimal strategy in (4.23), is tangent to the CB's highest possible isoloss curve appertaining to (4.36).

For the purpose of visual representation of the results, we employ parameter values used in Morris and Shin (2018): $\omega=\alpha=\gamma=1$. Upper left panel in Figure 4.1 represents the original no-acquisition case explored by Morris and Shin, which is

\footnotetext{
${ }^{18}$ See Appendix D.3 for a characterisation of the solution to the minimisation problem.
} 

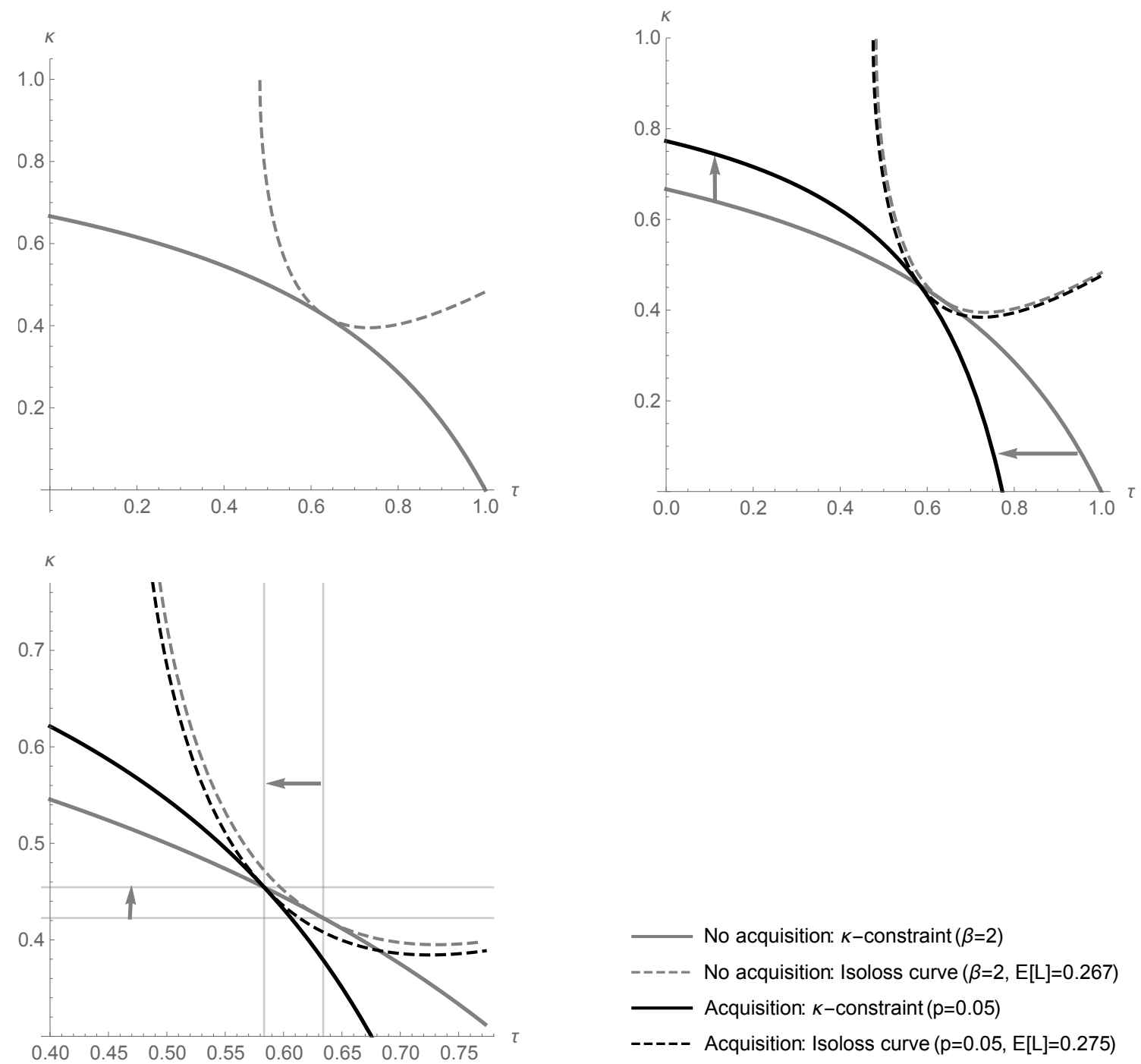

Figure 4.1: M-S versus private information acquisition. The $\kappa$-function and the CB's isoloss for $\omega=\alpha=\gamma=1$

calculated for an exogenously given ${ }^{19} \beta=2$. The solution in the original model is $\tau_{\mathrm{M}-\mathrm{S}}^{*}=0.63$ and $\kappa_{\mathrm{M}-\mathrm{S}}^{*}=0.42$, which corresponds to CB's expected loss being equal to 0.267 (where subscript $M-S$ stands for Morris-Shin case). Upper right panel overlays the present model with private information acquisition on top of the original model results. The present case is calculated for $\omega=\alpha=\gamma=1$ and $p=0.05$, which together ${ }^{20}$ with $\tau_{\text {acq }}^{P}=0.58$ (where subscript acq stands for $\beta$-acquisition case) yields an endogenous $\beta_{\mathrm{acq}}^{P}=2$. Thus, by choosing the information cost to be $p=0.05$,

\footnotetext{
${ }^{19}$ Morris and Shin choose $\omega=1$ as an approximation of the case in which market participants are almost entirely focused on matching the CB's action $g$. Apart from simplicity, their choice of values for $\alpha, \gamma$, and $\beta$ is seemingly completely arbitrary.

${ }^{20}$ It should be noted that $\tau_{\text {acq }}^{P}=0.58$ minimises the CB's expected loss, given $\omega=\alpha=\gamma=1$ and $p=0.05$. The value of $p$ was chosen so that it lies below $p_{1}=0.17$ and so that the loss-minimising $\tau_{\text {acq }}^{P}$ would correspond to $\beta_{\text {acq }}^{P}=2$.
} 
we made the two numerical examples directly comparable at the two equilibrium tangency points, as the quality of private information is numerically the same in both cases. Any point on the $\kappa_{\text {acq }}^{*}$-curve that is not the tangency point $\left(\tau_{\text {acq }}^{P}, \kappa_{\text {acq }}^{P}\right)$ corresponds to $\beta_{\text {acq }} \neq 2$, since endogenous $\beta^{*}$ varies with $\tau$.

First of all, it should be noted that in an information-acquisition scenario, the above numerical example leads to a P-NE at $\left(\tau_{\text {acq }}^{P}, \kappa_{\text {acq }}^{P}\right)$. The upper right figure demonstrates how presence of information acquisition can have a restrictive effect on the CB's decision making. Namely, that in the exogenous- $\beta$ M-S case the CB can commit to any value of $\tau \in(0,1)$ should the CB find it optimal to do so; whereas in the $\beta$-acquisition case the $\mathrm{CB}$ is restricted ${ }^{21}$ to $\tau \in(0,0.77)$ if the $\mathrm{CB}$ wishes to maintain a positive level of investment in private information acquisition $(0.77<\tau$ corresponds to $\beta=0$ ). The lower left figure (which is a close-up of the upper right figure) shows that the $\kappa_{\text {acq }}^{*}$-constraint is steeper than its M-S counterpart and the highest achievable isoloss curve is lower than that in the M-S case. The tangency point is at $\tau_{\text {acq }}^{P}=0.58$ and $\kappa_{\text {acq }}^{P}=0.45$ which corresponds to $E[L]=0.275$. Compared to the M-S case, it is optimal for the CB to commit to a lower $\tau$, which results in individual agents choosing a higher $\kappa$ and the CB suffering a greater expected loss, while the underlying quality of agents' private signals are the same in both cases.

We have already ascertained that the reflection problem is exacerbated in the presence of private information acquisition. By committing to a lower $\tau$, compared to the M-S case, the CB is able to achieve a higher equilibrium $\kappa$ and, hence, boost the accuracy of the market signal. As a result, the informativeness of the market signal is higher in the $\beta$-acquisition case: the accuracy of the average action as a forecast of $\theta$ is equal to 3 in the M-S case and 3.36 in the case with endogenous $\beta$. The benefit of higher information accuracy is, however, insufficient to offset the negative impact of a lower $\tau$ on the expected loss. As apparent from (4.36):

$$
E[L]=\tau^{2}(1-\kappa)^{2} \cdot \frac{1}{\alpha}+(1-\tau)^{2} \cdot \frac{1}{\gamma}
$$

a decrease in $\tau$ means that the CB is relying more on its imperfect signal $z$ (term in $\gamma$ increases in absolute terms) and less on the market signal (term in $\alpha$ decreases in absolute terms). An increase in $\kappa$ means that the individual agents place less weight on the public signal $y$, which, in turn, means that the market signal is less noisy (term in $\alpha$ in (4.36) decreases further in absolute terms). The effect on the $\gamma$-term

\footnotetext{
${ }^{21}$ The restriction is implied by the condition for existence of a P-NE: $0<p<p_{1}$. With $\alpha=\omega=1$, the critical price is $p_{1}=(1-\tau)^{2}$, and exogenously given $p=0.05$ satisfies the condition for existence of a $\mathrm{P}-\mathrm{NE}$ for $0<\tau<0.77$.
} 
dominates in this numerical example.

In the above numerical example $(\omega=\alpha=\gamma=1$ and $p=0.05)$, private information acquisition does not take place for some values of the policy parameter $\tau$. Namely, there is no information acquisition for $\tau$ values that violate the condition $0.05<(1-\tau)^{2}$, which is the $0<p<p_{1}$ condition evaluated at $\omega=\alpha=1$ and $p=0.05$. Next we consider a numerical example in which individual agents choose a positive $\beta^{*}$ for all values of $\tau$. Firstly, we choose a value of $\omega$ that is not equal to unity, as in the previous example. Setting $\omega=1$ precludes the possibility of there being a parameter value combination for which $0<\beta^{*}$ for $0<\tau<1$. The reason for this being that $\omega=1$ leads to the EDoC equal to just $\tau$, and, as apparent from the expression for $\beta^{*}$ in (4.20), the closer $\tau$ gets to unity, the closer the term in $\alpha$ is to $-\infty$. If agents are solely interested in matching their action with the CB's instrument adjustment and the CB commits to putting the full weight on the market signal, the incentive to acquire a heterogenous private signal is eliminated. Restricting $\omega$ to $(0,1)$ ensures that a price $p$ exists for which agents find it worthwhile to purchase a private signal regardless of the policy parameter setting. Thus, we arbitrarily choose $\omega=0.8$ which, together with $\alpha=\gamma=1$ yields $p_{1}=(1-0.8 \tau)^{2}$. In the limit, as $\tau \rightarrow 1, p_{1}=0.04$. To satisfy the purpose of this numerical example we choose $p=0.02$ so that $p<p_{1}$. The graphic representation of this example is presented in Figure 4.2.

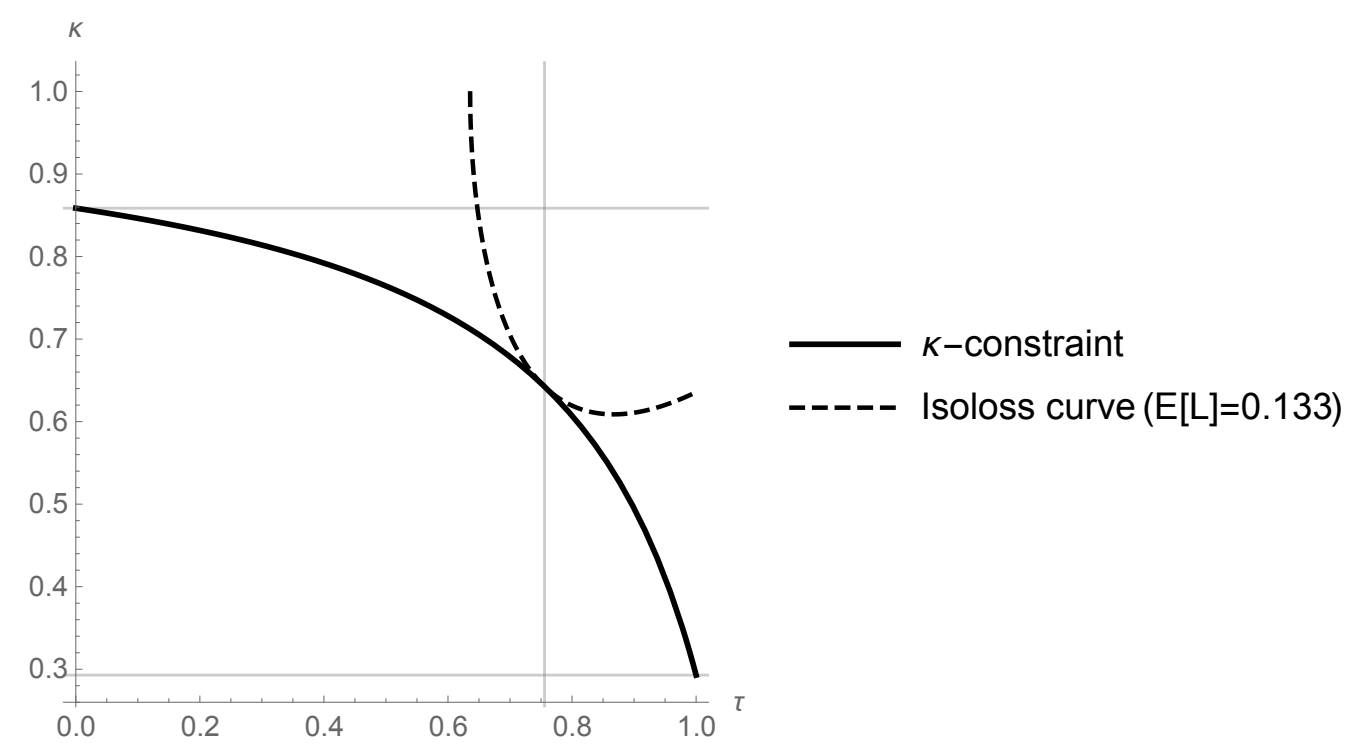

Figure 4.2: Private information acquisition. The $\kappa$-function and the CB's isoloss for $\alpha=\gamma=1, \omega=0.8$ and $p=0.02$.

As is apparent from Figure 4.2, the $\kappa$-weight is strictly positive for all $\tau$ and 
lies in the interval $(0.28,0.86)$. Even as $\tau$ approaches unity, individual agents still find it worthwhile to place a positive weight on their private signals, because it aids alignment of actions with the fundamental. As $\tau$ approaches zero, the scenario approaches the $\tau=0$ special case discussed in Section 4.2.2. In fact, the vertical intercept of the $\kappa$-constraint curve is $\hat{\kappa}_{i}$ from (4.6) following the substitution for $\beta_{i}=\hat{\beta}$ from (4.9):

$$
\left.\hat{\kappa}_{i}\right|_{\beta_{i}=\hat{\beta}}=1-\alpha \sqrt{p}
$$

and evaluated at $\alpha=1$ and $p=0.02$. The intercept moves in the direction of the plot origin $(0,0)$ with any increase in either $\alpha$, or $p$, or both. An increase in either or both parameters reduces the equilibrium $\hat{\beta}$, which, in turn, reduces the weight $\hat{\kappa}$ that agents place on their private signals.

Having characterised information use, information acquisition and CB's policy decision in a P-NE, we next turn to solving the present model for when no private information acquisition is taking place.

\subsubsection{Solving for non-purchasing equilibria (N-NE)}

When the combination of parameter values and policy setting is such that market participants do not find it worthwhile to invest in private information, the public signal $y$ becomes the only source of information about the state variable available to the market. In the absence of private information, individual agents' best response is just equal to the public signal:

$$
a_{i}=y,
$$

which means that the market-based signal reveals $y$ perfectly: $\bar{a}=y$. This corresponds to $\kappa=0$, which, in turn, implies a monetary policy rule that is a weighted average of signals $y$ and $z$. Another implication of $\kappa=0$ is that the reflection problem does not arise in a non-purchasing setting, since the CB's choice of $\tau$ does not affect agent $i$ 's best-response weight. Substituting $\kappa=0$ into CB's expected loss in (4.36) and minimising the resulting expression with respect to the policy parameter yields:

$$
\tau^{N}=\frac{\alpha}{\alpha+\gamma},
$$

where superscript $N$ stands for non-purchasing. Thus, it is optimal for the CB to set $g$ equal to its expectation of $\theta$, conditional on $y$ as well as $z$; and the coefficients placed on $y$ and $z$ are, in fact, Bayesian weights. Commitment to $\tau=\tau^{N}$ corresponds 
to an expected loss given by:

$$
E[L]_{\beta=0, \tau=\tau^{N}}=\frac{1}{\alpha+\gamma}
$$

which is strictly decreasing in both $\alpha$ and $\gamma$ (in absolute terms), as higher quality of information allows the $\mathrm{CB}$ to form a more accurate forecast of $\theta$.

As already discussed above, the $\mathrm{CB}$ is able to induce a N-NE in the market by committing to a policy rule that is not necessarily optimal for the purpose of minimising the CB's expected $\operatorname{loss}^{22}$. However, there exist parameter value combinations for which a N-NE is, in fact, associated with a loss-minimising rule captured by the $\tau$-setting in (4.40). Evaluating $p_{1}$ in (4.22) at $\tau=\tau^{N}$ from (4.40) yields the condition for existence of N-NE with optimal commitment:

$$
p_{2} \leq p
$$

where:

$$
p_{2}=\frac{1}{\alpha^{2}} \cdot\left(1-\frac{\alpha \omega}{\alpha+\gamma}\right)^{2}
$$

The above condition together with expression (4.40) constitute Proposition 4.2.

Proposition 4.2: Given agents make a payoff-maximising decision about private information acquisition and the information cost $p$ is greater or equal to $p_{2}$, a nonpurchasing equilibrium exists and it is optimal for the central bank to commit to $\tau=\tau^{N}$. The reflection problem does not arise.

The critical price value in (4.42) is strictly positive for all values of $\omega, \alpha$, and $\gamma$. This means that there always exists a non-empty set of sufficiently low prices $p$ for which a N-NE with optimal commitment does not exist. In other words, there exists no combination of values of $\omega, \alpha$, and $\gamma$ such that a N-NE with optimal commitment exists for all $0<p$.

The critical value $p_{2}$ is strictly decreasing in $\omega$. Suppose, for a moment, that $p=p_{2}$ and individual agents find it just optimal to not invest in private information acquisition $(\beta=\kappa=0)$. A marginal decrease in $\omega$ corresponds to each agent being marginally more concerned with aligning its action with the fundamental than with the policy instrument. Endogenously, that leads to a weaker equilibrium degree of

\footnotetext{
${ }^{22}$ From (4.31), if $\frac{(1-\omega)^{2}}{\alpha^{2}}<p<\frac{1}{\alpha^{2}}$ holds, the CB can induce a N-NE by setting $\frac{1-\alpha \sqrt{p}}{\omega}<$ $\tau<1$.
} 
coordination. Accordingly, relative benefit of observing a private signal increases, the range of prices for which a N-NE exists becomes narrower, and now the actual information price $p$ is below the new critical value $p_{2}^{\prime}$. With $p<p_{2}^{\prime}$ individual agents find it just worthwhile to invest in a positive value of $\beta$.

Since we are unable to arrive at a tractable solution for $\tau^{P}$ and $\kappa^{P}$ in the P-NE case, we refrain from commenting on how the value of $p_{2}$ relates to the value of $p_{1}$ evaluated at $\tau=\tau^{P}$. Comparing the two critical price values would allow us to characterise the cases in which both types of $\mathrm{NE}$ exist ${ }^{23}$ and, if so, whether one type of NE is dominant due to being associated with lower expected loss for the CB. However, having arrived at a tractable solution for $\tau^{N}$, we are able to take a closer look at agent $i$ 's expected equilibrium payoff.

\subsubsection{Agent $i$ 's expected equilibrium payoff in $\mathrm{N}-\mathrm{NE}$}

In discussing agent $i$ 's expected payoff in the absence of private information acquisition, we follow the same approach as in Section 4.2.3.3. Setting $\beta_{i}=\beta=0$ in (4.25) yields:

$$
E\left[u_{i}\right]_{\beta=0}=-\frac{\left(1-2 \omega \tau+\omega \tau^{2}\right)}{\alpha}-\frac{\omega(1-\tau)^{2}}{\gamma} .
$$

Since agents are not purchasing idiosyncratic information, there is no dispersion loss or information cost. The remaining two terms are volatility loss and variance of $\phi$ loss. There is no secondary or tertiary effect because the best response weight $\kappa$ and private information quality are equal to zero and do not vary. Only the primary effects remain, which have already been discussed in relation to (4.24) above. It should be noted that expression in (4.43) is strictly increasing in $\tau$. Thus, similarly to the P-NE case, if $\tau$ were chosen by the $\mathrm{CB}$ so as to maximise agents' individual expected payoffs, it would be optimal to set $\tau=1$. However, based on the assumptions of our model ${ }^{24}$, the $\mathrm{CB}$ chooses to commit to $\tau=\tau^{N}$, where $\tau^{N}<1$ minimises its loss and does not maximise agents' payoffs.

Since we are able to arrive at an optimal value for the CB's policy parameter in a N-NE, we can further comment on the effect that a change in $\alpha$ or $\gamma$ has on the components of agent $i$ 's expected payoff. Substituting $\tau=\tau^{N}$ from (4.40) into (4.43) yields:

$$
E\left[u_{i}\right]_{\beta=0, \tau=\tau^{N}}=-\frac{1}{\alpha}+\frac{(\alpha+2 \gamma) \omega}{(\alpha+\gamma)^{2}}-\frac{\gamma \omega}{(\alpha+\gamma)^{2}}
$$

\footnotetext{
${ }^{23}$ One of the numerical examples uses a parameter value combination for which both types of NE exist and yield the same level of CB's expected loss. See Figure 4.5.

${ }^{24}$ Following Morris and Shin (2018), we assume that the CB's expected loss differs from the representative agent's expected payoff.
} 
where the first and the second terms correspond to volatility loss and the third term is the variance of $\phi$ loss.

As far as volatility loss is concerned, $\operatorname{when}^{25} p_{2} \leq p$ and $\tau=\tau^{N}$, an increase in $\alpha$ reduces volatility loss through two channels. On the one hand, since $\bar{a}=y$, an increase in the quality of the public signal directly translates into an increase in the accuracy of the average action as a predictor of the state variable, which allows for better alignment of $\bar{a}$ and $\theta$. On the other hand, any increase in the accuracy of $\bar{a}$ as a market signal relative to $z$ leads to the CB putting less weight on its private information and more weight (higher $\tau$ ) on the average action. Taking into account optimal commitment to $\tau=\tau^{N}$ reinforces the primary effect that $\alpha$ has on volatility. For individual agents, an increase in $\tau$ means that aligning their actions with the unobservable $z$ becomes less important. Both effects work in the same direction, reducing volatility loss.

An increase in $\gamma$ is an increase in the quality of $z$ relative to the market signal, therefore, the CB commits to a rule that relies more on $z$ and less on $\bar{a}$. Having to matching instrument adjustment that follows a rule with a higher weight on $z$, agents suffer an increase in volatility loss term. An increase in $\omega$ creates two effects that work in the opposite direction. The explicit aim of matching $\theta$ becomes less important, which reduces the coefficient on volatility; while the implicit aim of matching $z$ gains relative importance, which increases the volatility loss term. The former effect dominates, thus, the volatility loss term is strictly decreasing in $\omega$.

We now consider how the remaining term in agent $i$ expected payoff is affected by parameters. The variance of $\phi$ loss terms is strictly decreasing in $\alpha$, since an increase in the quality of the public signal is equivalent to an increase in the quality of the market signal observed by the CB. The latter commits to a higher weight on the market signal and a lower weight on $z$, thereby, reducing the loss agents suffer from being unable to forecast $\phi$. On the one hand, an increase in $\gamma$ has the same indirect effect that works through $\tau^{N}$ but has the opposite sign. On the other hand, there is also the primary effect of reducing the variance of $\phi$. The former offsets the latter and, as a result, the variance loss term is strictly increasing $\phi$. Any increase in $\omega$ increases the volatility loss, as agents' endogenous aim of matching $z$ becomes relatively more important.

Once we put the two loss terms together (and take into account the minus sign),

${ }^{25}$ From $(4.42), p_{2}=\frac{1}{\alpha^{2}} \cdot\left(1-\frac{\alpha \omega}{\alpha+\gamma}\right)^{2}$. 
we are able to comment on the total effect of changes in $\alpha, \gamma$, and $\omega$ on agent $i$ 's expected payoff. An increase in the quality of public information, as represented by $\alpha$, has positive effect on the expected payoff, as both volatility and variance (of $\phi)$ are reduced. The opposite is true for the quality of CB's private information $\gamma$ - expected payoff is decreasing in $\gamma$, as both volatility and variance are increased. An increase in $\omega$ reduces volatility loss and increases variance, and the former effect dominates. Thus, agent $i$ 's expected payoff is strictly decreasing in $\omega$.

Having characterised agent $i$ 's expected payoff and CB's expected loss in N-NE, we turn to a series of numerical examples that demonstrate how P-NE and N-NE relate to each other.

\subsubsection{Numerical examples of N-NE}

What follows is a discussion of several numerical examples of P-NE and N-NE that help illustrate a number of policy considerations. Figure 4.3 is a modified version of the top right panel in Figure 4.1. Using just the curves for the $\beta$-acquisition case calculated for $\omega=\alpha=\gamma=1$ and $p=0.05$, we extend the graph by adding the section of the $\kappa$-constraint that corresponds to absence of private information acquisition: $\kappa=0$ for $\tau \in[0.77,1)$. We already know that in this numerical example the highest achievable isoloss curve is tangential to the $\kappa$-constraint at $\tau^{*}=0.58$, which is a P-NE with $E\left[L^{\mathrm{CB}}\right]=0.275$. Figure 4.3 demonstrates that by adjusting its policy parameter to $\tau=0.9$, for example, the CB is able to induce a N-NE, in which individual agents no longer find it optimal to purchase private signals. At $\tau=0.9$, the $\kappa$-constraint is intersected by an isloss curve that lies below the P-NE isoloss curve identified above. This is the case for any N-NE-inducing value of $\tau$ in this example, making commitment to such policy rules not credible.

In contrast to Figure 4.3, Figure 4.4 presents a numerical example of a parameter value combination for which the highest attainable isoloss curve corresponds to a N-NE. When $\omega=\alpha=\gamma=1$ and $p=0.5$, the isoloss is tangential to the horizontal section of the $\kappa$-constraint at $\tau=0.5$. Although the $\mathrm{CB}$ can induce a P-NE by setting $\tau \in(0,0.29)$, that would result in a higher expected loss (lower isoloss curve). Figure 4.5 presents a numerical example of a special case in which the highest possible isloss curve is tangential to the $\kappa$-constraint at two points. When $\omega=\alpha=\gamma=1$ and $p=0.315$, the isoloss is tangential to the concave section of the $\kappa$-constraint at $\tau=0.4$ and tangential to the horizontal section at $\tau=0.5$. The former value of the policy parameter is the CB's optimal response in the presence of private information acquisition, and the latter is the optimal policy setting in the absence of information acquisition. Interestingly, both $\mathrm{NE}$ are characterised by the 


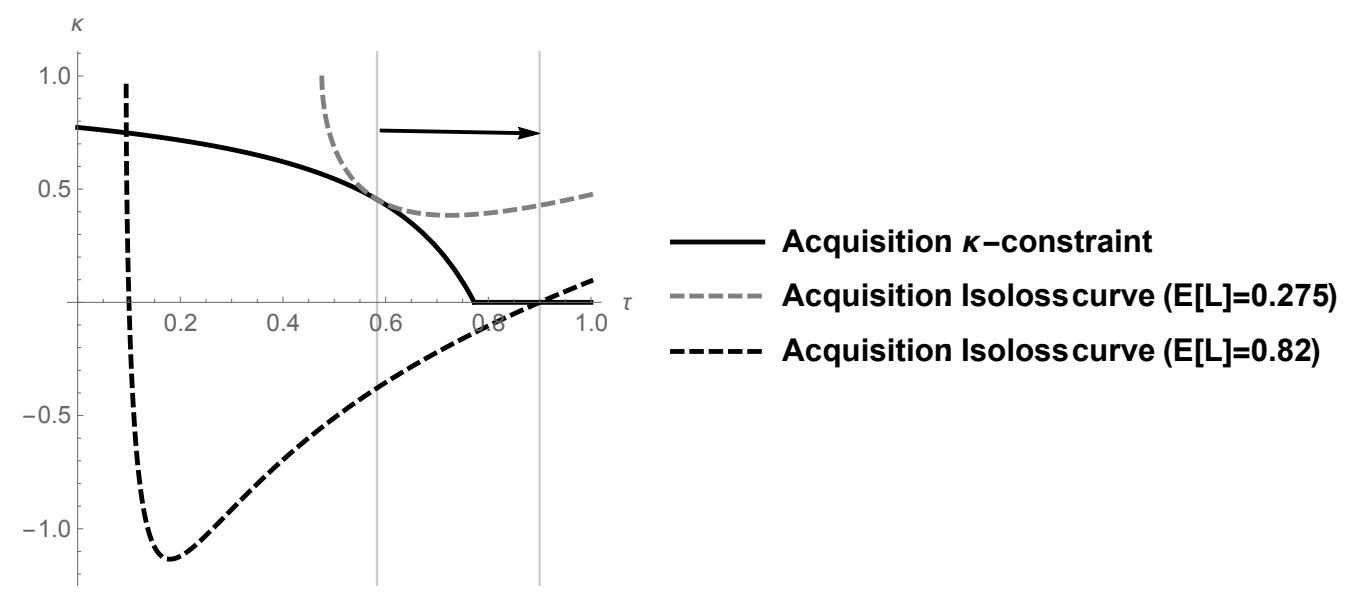

Figure 4.3: Private information acquisition at $\tau=0.58$ and $\tau=0.9$. The $\kappa$-function and the CB's isoloss for $\omega=\alpha=\gamma=1$

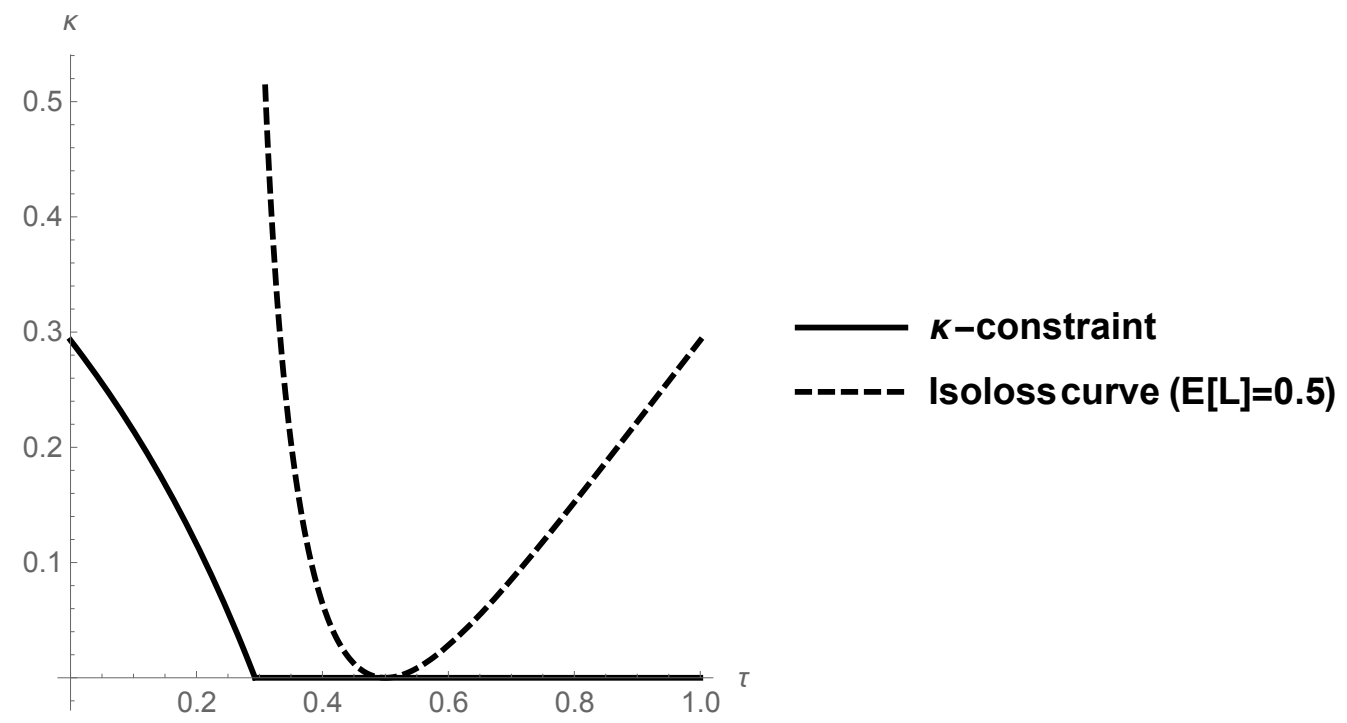

Figure 4.4: Private information acquisition. The $\kappa$-function and the CB's isoloss for $\omega=\alpha=\gamma=1$ and $p=0.5$

same level of CB's expected loss, which leaves the CB indifferent between the two strategies. This numerical example $(\omega=\alpha=\gamma=1$ and $p=0.315)$ proves that the set of parameter combinations for which both NE types exist is not empty, which, in terms of critical price values, corresponds to $p_{2} \leq p_{1}$. Decreasing the value of $p$ below $p=0.315$ leads to a P-NE becoming the superior (and eventually the sole ${ }^{26}$ ) outcome, whereas increasing the value of $p$ above $p=0.315$ leads to a N-NE becoming the superior outcome. Figure 4.6 demonstrates that a reduction in information

\footnotetext{
${ }^{26} \mathrm{~A}$ P-NE becomes the sole outcome when the $\kappa$-constraint shifts so far to the right, that the point where it becomes horizontal lies to the right of the tangency point between an isoloss curve and the $\tau$-axis (the point that corresponds to the N-NE). This is the case for $0<p<p_{2}$ or $0<p<\frac{1}{2}$ for $\omega=\alpha=\gamma=1$.
} 


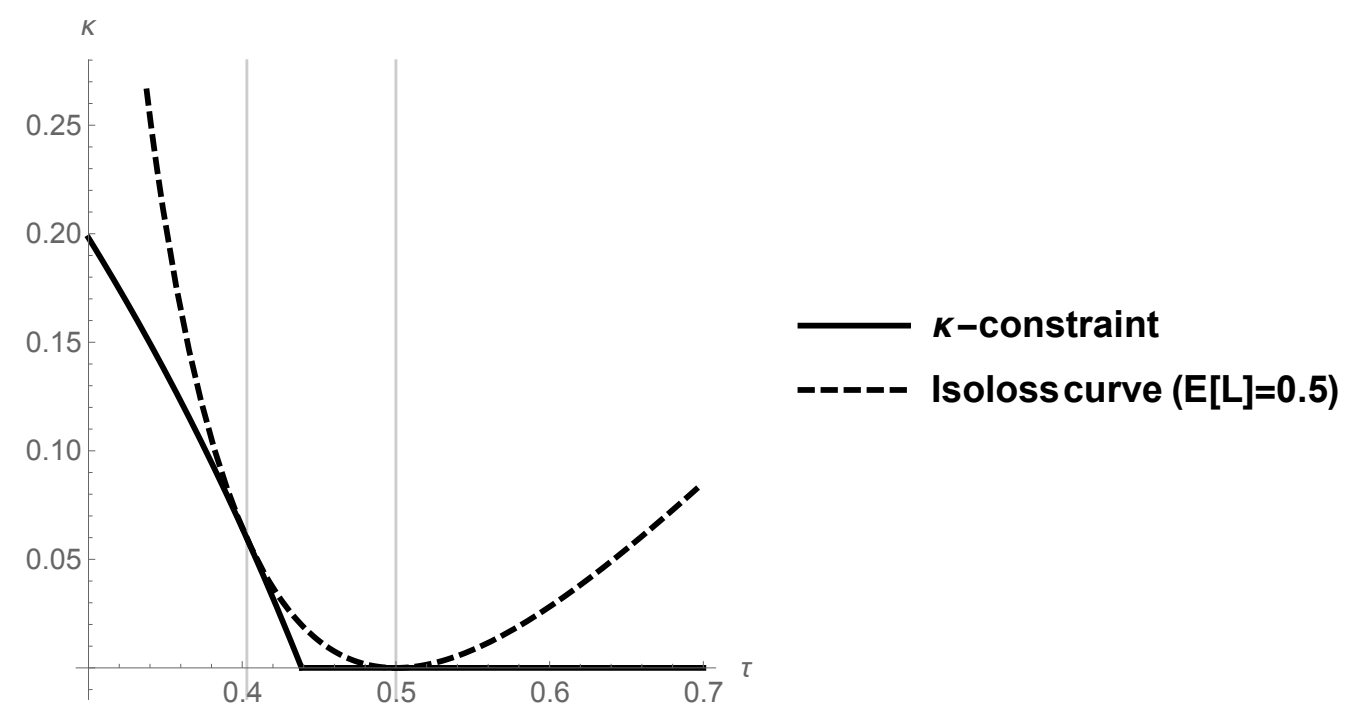

Figure 4.5: Private information acquisition. The $\kappa$-function and the CB's isoloss for $\omega=\alpha=\gamma=1$ and $p=0.315$

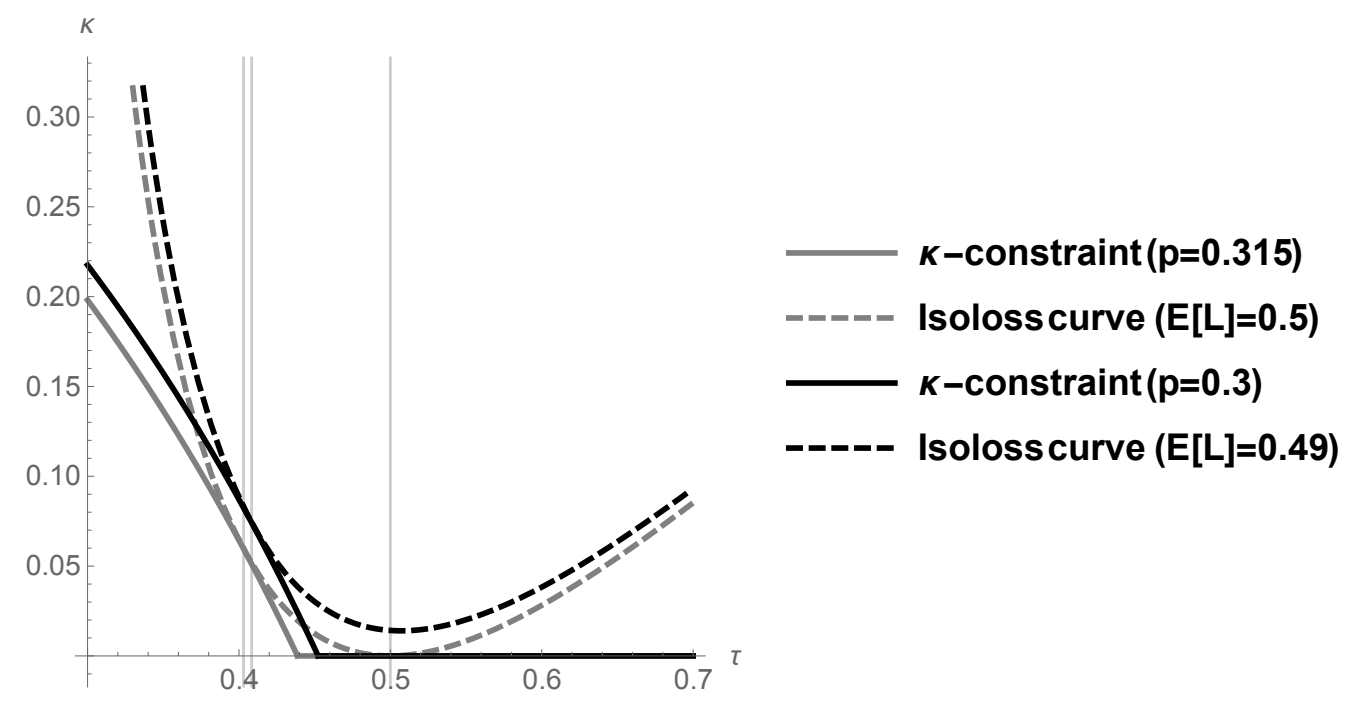

Figure 4.6: Private information acquisition. The $\kappa$-function and the CB's isoloss for $\omega=\alpha=\gamma=1$ and $p \in\{0.3,0.315\}$

cost from $p=0.315$ to $p=0.3$ leads to a rightward shift in the $\kappa$-constraint ${ }^{27}$; the isoloss curve that is tangential to the horizontal portion of the $\kappa$-constraint is now below the isoloss that is tangential to the non-zero part of the constraint (that corresponds to $p=0.3$ ). Thus, the $\mathrm{P}-\mathrm{NE}$ is superior in this case. Figure 4.7 shows the effect of a rise in $p$ from 0.315 to 0.35 . The $\kappa$-constraint shifts left, and the isoloss that is tangential to the non-zero part of it is below the isoloss that is tangential to the horizontal part. Thus, the N-NE is superior in this case.

\footnotetext{
${ }^{27}$ The shift is not parallel, as the change in $p$ affects the slope of the portion of the $\kappa$-constraint that corresponds to positive $\beta$ : the slope becomes less steep as $p$ falls.
} 


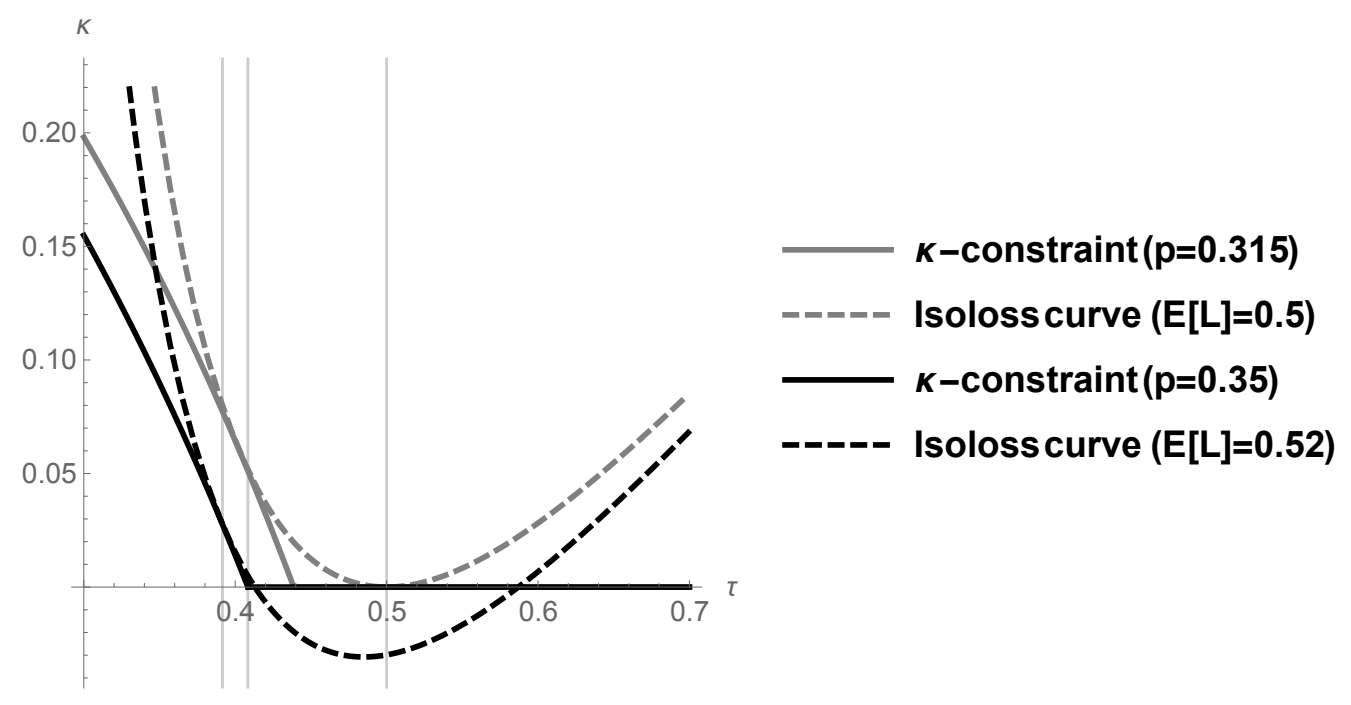

Figure 4.7: Private information acquisition. The $\kappa$-function and the CB's isoloss for $\omega=\alpha=\gamma=1$ and $p \in\{0.315,0.35\}$

This concludes our discussion of the signal value of the market aggregate and monetary policy in the context of private information acquisition. We proceed to the second part of this Chapter in which we explore a scenario with public information acquisition.

\subsection{Public information acquisition}

So far in this Chapter, we focused on private information acquisition. We extended the original Morris and Shin (2018) model by allowing individual agents to choose the quality of their idiosyncratic private signals. When agents chose for their private signals to have positive informational value, they incurred an information cost represented by a linear term. In what follows we explore a different variation of the Morris-Shin model. Namely, we look at the acquisition of public information.

\subsubsection{Model variation}

The model assumptions laid out in Section 4.2.1 remain largely the same, thus, here we only outline the differences in assumptions. Firstly, there is no longer any private information acquisition. Individual agents observe their private signals free of cost, and the quality of these signals is exogenously fixed at $\beta$.

Instead of agents simultaneously and privately deciding on the value of $\beta$, they are now simultaneously and privately deciding whether or not to purchase the pub- 
lic signal $y \cdot{ }^{28}$ Although the nature of signal $y$ changes as soon as we introduce an information cost, we continue to refer to it as the public signal for the purpose of consistency. In fact, Morris and Shin clarify that signal $y$ is referred to as 'public' because "the actual realisation of $y$ is common knowledge to all agents" (Morris and Shin, 2002, p. 1525). Thus, the term 'public' refers as much to information commonality as it does to its accessibility by agents. Under the costly information assumption, signal $y$ is no longer freely available, so it does not automatically become common knowledge to all agents, but instead it is common knowledge to all agents that choose to purchase $y$. In a symmetric purchasing equilibrium (P-NE), these two sets of agents coincide making the distinction immaterial. However, being able to allow for all but one agent to purchase $y$ (i.e. allowing for the two sets to differ by one agent) is paramount when deriving the necessary and sufficient conditions for the existence of P-NE.

The quality of the public signal is exogenously given and fixed at $\alpha$. In order to observe $y$, individual agents can choose to pay a fixed price $p_{y}$. In the previously discussed private information acquisition scenario, $p$ was the price per unit of signal precision, whereas here we assume $p_{y}$ to be a one-off flat fee for access to a signal of given quality ${ }^{29}$. In the earlier Sections, information acquisition was characterised by a point on the continuum $0 \leq \beta$, where $0<\beta=\beta^{*}$ corresponded to a particular $\mathrm{P}-\mathrm{NE}$ from the set of $\mathrm{P}-\mathrm{NE}$ and $\beta=0$ corresponded to the N-NE. A given value of parameter $p$, if it lay below the critical value $p_{1}$, lead to an equilibrium choice of $\beta^{*}$ and $\tau^{P}$ that defined a particular P-NE; and, if the given value of $p$ was equal to or lay above the critical value $p_{2}$, it lead to the single N-NE outcome defined by $\beta=0$ and $\tau=\tau^{N}{ }^{30}$ In contrast to that, the flat-fee assumption used here leads to a discrete characterisation of information acquisition $-y$ is either purchased or not. The dichotomous choice corresponds to a single P-NE and a single N-NE. Thus, a given value of $p_{y}$ produces either the $\mathrm{P}-\mathrm{NE}$ or the $\mathrm{N}-\mathrm{NE}^{31}$.

In order to characterise the conditions for existence of NE of either type (purchasing or non-purchasing), we look at whether an individual agent would choose

\footnotetext{
${ }^{28}$ This affects Step 2 in the sequence of events outline in Section 4.2.1, leaving the remaining steps unchanged.

${ }^{29}$ While the treatment of private information acquisition in this Chapter is analogous to 'Linear Cost' Sections in the previous Chapter, the analysis of public information acquisition here parallels the 'Fixed Cost' Sections in the previous Chapter.

${ }^{30}$ It should be noted that, since the numerical example that corresponds to Figure 4.5 proves that there exists a set of value combination for $\alpha, \gamma, \omega$, and $p$ such that the CB is indifferent between inducing a P-NE and a N-NE, the following can be true: if $p_{2}<p_{1}$ and the given value of $p$ satisfies $p_{2} \leq p<p_{1}$, it leads to either a particular P-NE or the N-NE.

${ }^{31}$ As will be demonstrated in later Sections, for some values of $p_{y}$, both NE exist, however, there exists no value of $p_{y}$ for which no NE exists at all.
} 
to deviate in its information acquisition decision from the rest of the population. Namely, we determine the necessary and sufficient conditions for agent $i$ to find it optimal to purchase $y$, given the knowledge that all other agents also choose to purchase $y$. The conditions that, if met, ensure that a representative agent has no incentive to deviate from a purchasing population are also the necessary and sufficient conditions for the existence of a P-NE. We repeat the analysis for N-NE by deriving the conditions under which agent $i$ does not deviate from a non-purchasing population.

In formal terms we take the payoff function from (4.3) and modify it in the following way:

$$
u_{i}=-\omega\left(a_{i}-g\right)^{2}-(1-\omega)\left(a_{i}-\theta\right)^{2}-\nu_{i} p_{y},
$$

where $\nu_{i} \in\{0,1\}$ and $p_{y}$ is the flat fee for observing $y$. Agent $i$ is facing a discrete choice of either purchasing the public signal $\left(\nu_{i}=1\right)$ or not purchasing the public signal $\left(\nu_{i}=0\right)$. We proceed to derive expected payoffs for (i) when agent $i$ deviates from the rest of the population i.e. $\nu_{i} \neq \nu_{j}=\nu_{k}$ for $i \neq j$ and $\forall j, k$; and (ii) when agent $i$ does not deviate from the rest of the population i.e. $\nu_{i}=\nu_{j}$ and $\forall i, j$. By comparing the payoffs we work out a critical price value at which agent $i$ is indifferent between deviating and not deviating. First, in Section 4.3.2, we perform the comparison for when agent $i$ is considering deviating from a purchasing population $\left(\nu_{j}=1, i \neq j\right)$ and obtain a value for $p_{\max }$, such that a P-NE exists for $0<p_{y}<p_{\max }$. Then, in Section 4.3.3, we repeat the comparison for when agent $i$ is considering deviating from a non-purchasing population $\left(\nu_{j}=0, i \neq j\right)$ and obtain a value for $p_{\min }$, such that a N-NE exists for $p_{\min }<p_{y}$.

As far as monetary policy is concerned, we maintain the Morris and Shin (2018) assumption that the $\mathrm{CB}$ cannot access the public signal nor make it part of its instrument adjustment rule. All the other assumptions about the CB also continue to be maintained in this model variation. We look at policy considerations in the context of public information acquisition in Section 4.3.4.

\subsubsection{Solving for purchasing equilibria ( $\mathrm{P}-\mathrm{NE})$}

We begin by assuming that all agents are purchasing public information $\left(\nu_{j}=\right.$ $\left.\nu_{k}=1, \forall j, k\right)$, except for agent $i$ who is making a choice whether to deviate or not $\left(\nu_{i} \in\{0,1\}\right)$.

In a symmetric equilibrium, agent $i$ is not deviating and agent $i$ 's payoff is given 
by:

$$
u_{i}^{P}=-\omega\left[a_{i}^{P}-\tau \bar{a}^{P}-(1-\tau) z\right]^{2}-(1-\omega)\left(a_{i}^{P}-\theta\right)^{2}-p_{y},
$$

where superscript $P$ stands for purchasing, and the payoff is obtained by setting $\nu_{i}=1$ in (4.45) and substituting the CB's rule from (4.2). Agent $i$ 's best response to observing signals $x_{i}$ and $y$ as well as the average action in this case are identical to those already derived in Section 4.2.3.1. For consistency of notation in the discussion of this model variation, we add the superscript $P$ to agent $i$ 's best response in (4.5):

$$
a_{i}^{P}=\kappa^{P} x_{i}+\left(1-\kappa^{P}\right) y,
$$

average action in (4.13):

$$
\bar{a}^{P}=\kappa^{P} \theta+\left(1-\kappa^{P}\right) y,
$$

and the best-response coefficient ${ }^{32}$ in (4.16):

$$
\kappa^{P}=\frac{\beta(1-\omega \tau)}{\alpha+\beta(1-\omega \tau)}
$$

As already mentioned in Section 4.2.3.1, the fact that the best-response coefficient in (4.49) is strictly decreasing in the policy parameter $\tau$ is indicative of the reflection problem. In fact, the relationship between $\kappa^{P}$ and $\tau$ in (4.49) is identical to the result discussed in Morris and Shin (2018). Substituting (4.47)-(4.48) into (4.46), taking expectations of the resulting expression, and then substituting (4.49) yields the P-NE expected equilibrium payoff:

$$
E\left[u_{i}^{P}\right]=-\frac{\beta(1-\omega \tau)^{2}}{[\alpha+\beta(1-\omega \tau)]^{2}}-\frac{\left(1-2 \omega \tau+\omega \tau^{2}\right) \alpha}{[\alpha+\beta(1-\omega \tau)]^{2}}-\frac{\omega(1-\tau)^{2}}{\gamma}-p_{y},
$$

The expected payoff in (4.50) is analogous to (4.25), with the exception of the cost term that here is a fixed fee equal to $p_{y}$, rather than a linear term in $\beta$.

If agent $i$ decides to deviate from a purchasing population, the respective payoff is given by:

$$
u_{i}^{P d}=-\omega\left[a_{i}^{P d}-\tau \bar{a}^{P}-(1-\tau) z\right]^{2}-(1-\omega)\left(a_{i}^{P d}-\theta\right)^{2},
$$

where superscript $P d$ stands for deviating from a purchasing population. Without having access to public information, individual agent uses just the private signal when forming expectations, and, hence, $E_{i}[\theta]=E_{i}[z]=E_{i}[y]=E_{i}[\bar{a}]=E_{i}[g]=x_{i}$.

\footnotetext{
${ }^{32}$ As mentioned earlier, precision $\beta$ here is an exogenous parameter, whereas it is assumed to be a decision variable in (4.16).
} 
Thus, agent $i$ 's payoff-maximising action is:

$$
a_{i}^{P d}=x_{i}
$$

where $\kappa^{P d} \equiv 1$. The average action is unaffected by agents $i$ 's deviating response and remains equal to $\bar{a}^{P}$. Substituting (4.48) and (4.52) into (4.51), taking expectations, and then substituting (4.49) yields the expected payoff of a deviating agent:

$$
E\left[u_{i}^{P d}\right]=-\frac{1}{\beta}-\frac{\alpha \omega \tau^{2}}{[\alpha+\beta(1-\omega \tau)]^{2}}-\frac{\omega(1-\tau)^{2}}{\gamma},
$$

which differs from $E\left[u_{i}^{P}\right]$ in (4.50).

We have already identified the components of agent $i$ 's expected payoff and discussed a number of considerations for agent $i$ ' decision-making in Section 4.2.3.3, and we draw parallels with already established results in what follows. Agent $i$ 's decision whether or not to purchase public information has no effect on expected volatility loss or variance of $\phi$ loss $^{33}$. The former captures the average misalignment of agents actions and the state variable, whereas the latter reflects limited accuracy of CB's private information; and an isolated individual agent has no influence over either. However, expected dispersion loss is agent-specific, as it captures the variation between agent $i$ 's action and the average action. Thus, by deviating in the information acquisition choice and information use from the population, agent $i$ incurs a greater dispersion loss. An increase in misalignment between agent $i$ 's own action and the average action happens for two reasons. Firstly, without observing $y$ agent $i$ can no longer perfectly forecast the common term in other agents' actions, namely, $\left(1-\kappa^{P}\right) y$. Secondly, having access to only one signal, agent $i$ forms a less accurate forecast of $\theta$ relative to the rest of the population, which impedes agent $i$ 's ability to match the $\kappa^{P} \theta$ term of the average action. For the same reason, agent $i$ is also less able to align its action with the $z$-component of CB's rule, which translates into additional loss.

An agent that chooses to deviate from a purchasing population expects to experience a negative and a positive effect on its payoff. On the one hand, not observing $y$ reduces agent $i$ 's ability to match the CB's instrument and the fundamental, which reduces the expected payoff. On the other hand, by not purchasing public information, agent $i$ avoids incurring any information costs. Thus, an individual agent has no incentive to deviate from a purchasing population when the benefit of acquiring $y$ outweighs the cost of doing so. Formally, agent $i$ 's optimal strategy with regard

\footnotetext{
${ }^{33}$ See expression (4.24) in Section 4.2.3.3 or expressions (D.27) and (D.28) in Appendix D.2.
} 
to purchasing $y$ is as follows:

$$
\begin{cases}\text { if } p_{y}<p_{\max }, & \nu_{i}=1 \\ \text { if } p_{y}=p_{\max }, & \nu_{i} \in\{0,1\} \\ \text { if } p_{\max }<p_{y}, & \nu_{i}=0,\end{cases}
$$

where $p_{\max }$ is the critical value for which $E_{i}\left[u_{i}^{P}\right]=E_{i}\left[u_{i}^{P d}\right]$. Since a P-NE occurs when all agents find it worthwhile to purchase $y$, a P-NE exists for:

$$
0<p_{y}<p_{\max }
$$

where:

$$
p_{\max } \equiv \frac{\alpha(\alpha+\beta)}{\beta[\alpha+\beta(1-\omega \tau)]^{2}} .
$$

The identified value of $p_{\max }$ is strictly positive. In other words, for any combination of values of $\alpha, \beta, \omega$, and $\tau$ there exists a sufficiently low $p_{y}$ for which a P-NE exists. $p_{\max }$ is strictly decreasing in $\beta$ and strictly increasing in $\omega$ and $\tau .{ }^{34} p_{\max }$ is decreasing in $\alpha$ when the following set of conditions is satisfied:

$$
\left\{\begin{array}{l}
\frac{1}{2}<\omega<1 \\
\frac{1}{2 \omega}<\tau<1 \\
0<\beta<\frac{\alpha(1-2 \omega \tau)}{1-\omega \tau}
\end{array}\right.
$$

and increasing in $\alpha$ otherwise ${ }^{35}$.

The critical value $p_{\max }$ is affected by changes in $\alpha, \beta, \omega$, and $\tau$ directly (primary effects) as well as through $\kappa^{P}$ (secondary effects). Changes in $\alpha, \beta, \omega$, and $\tau$ simultaneously affect $E_{i}\left[u_{i}^{P}\right]$ and $E_{i}\left[u_{i}^{P d}\right]$. If the net effect is such that the difference between the two expected payoff, in absolute terms, increases, then $p_{\max }$ also increases, and vice versa.

As far as primary effects are concerned, an increase in $\beta$ reduces dispersion loss in both cases - agent $i$ not deviating $(P)$ and deviating $(P d)$ from a purchasing population - by reducing the variance of idiosyncratic noise in private information.

\footnotetext{
${ }^{34}$ In the absence of any state-contingent information about the realisation of the noise $\phi$ in $z$, agent $i$ is unable to offset the $(1-\tau) \phi$ component of the CB's rule. Hence, agent $i$ 's decision whether or not to purchase public information has no effect on the loss associated with the variance of $\phi$, and the identified value for $p_{\max }$ is independent of $\gamma$.

${ }^{35}$ It should be noted that $0<\omega \leq \frac{1}{2}$ is a sufficient condition for $p_{\max }$ to be increasing in $\alpha$.
} 
An increase in $\alpha$ reduces $P d$-dispersion loss by facilitating alignment between a deviating agent's action and the $y$-component of other agents' actions. An increase in either $\omega$ or $\tau$ increases the weight on alignment between a deviating agent's action and the $y$-component of other agents' actions in the $P d$-payoff, thereby increasing the $P d$-dispersion loss.

As already discussed in relation to expression (4.23), the best-response weight of an agent that purchases both signals $\left(\kappa^{P}\right)$ is strictly increasing in $\beta$ and strictly decreasing in $\alpha$ (due to a change in the relative precision of $x_{i}$ and $y$ ), as well as strictly decreasing in $\omega$ and $\tau$ (due to an increase in endogenous coordination). Secondary effects of changes in $\alpha, \beta, \omega$, and $\tau$ on the two expected payoffs work through $\kappa^{P}$ in the following way. On the one hand, an increase in $\kappa^{P}$ increases $P$-dispersion, as more weight is placed on the misalignment of the idiosyncratic term in $a_{i}^{P}$ and the $\theta$ component of $\bar{a}^{P}$. On the other hand, an increase in $\kappa^{P}$ decreases $P d$-dispersion, as the deviating agent is better able to match the average action, in which less weight is placed on $y$ (which the deviating agent does not observe).

Since the critical value $p_{\max }$ in (4.54) contains $\tau$, the full characterisation of the condition for existence of P-NE ultimately depends on the CB's choice of its policy parameter. However, before we discuss monetary policy considerations, we first turn to deriving the condition for existence of N-NE.

\subsubsection{Solving for non-purchasing equilibria (N-NE)}

We now assume that no agent is purchasing public information $\left(\nu_{j}=\nu_{k}=0, \forall j, k\right)$, except for agent $i$ who is making a choice whether to deviate or not $\left(\nu_{i} \in\{0,1\}\right)$.

In a symmetric equilibrium, agent $i$ is not deviating in its information acquisition strategy and agent $i$ 's payoff is given by:

$$
u_{i}^{N}=-\omega\left[a_{i}^{N}-\tau \bar{a}^{N}-(1-\tau) z\right]^{2}-(1-\omega)\left(a_{i}^{N}-\theta\right)^{2},
$$

where superscript $N$ stands for non-purchasing. As in the $P d$-case, agent $i$ finds it optimal to set the action equal to the only signal agent $i$ observes:

$$
a_{i}^{N}=x_{i}
$$

where $\kappa^{N} \equiv 1$. The fact that $\kappa^{N}$ is independent of the policy parameter $\tau$ signifies 
the absence of the reflection problem in a N-NE. The average action is then:

$$
\bar{a}^{N}=\theta
$$

It is apparent from (4.58) that, in a N-NE, the average action (that acts as a market signal to the $\mathrm{CB}$ ) becomes a perfectly accurate predictor of the state variable. Substituting (4.57) and (4.58) into (4.56) and taking expectations of the resulting expression yields:

$$
E\left[u_{i}^{N}\right]=-\frac{1}{\beta}-\frac{\omega(1-\tau)^{2}}{\gamma}
$$

Since the average action is perfectly aligned with the state variable, there is no volatility loss in (4.59). Agent $i$ 's expected payoff consist of the dispersion loss and variance of $\phi$ loss terms. The former is just the variance of the forecast error of $x_{i}$ as a signal of $\theta$.

If agent $i$ deviates from a non-purchasing population, then agent $i$ payoff is given by:

$$
u_{i}^{N d}=-\omega\left[a_{i}^{N d}-\tau \bar{a}^{N}-(1-\tau) z\right]^{2}-(1-\omega)\left(a_{i}^{N d}-\theta\right)^{2}-p_{y} .
$$

The payoff in (4.60) is maximised by setting the action equal to:

$$
a_{i}^{N d}=\omega\left\{\tau E_{i}\left[\bar{a}^{N}\right]+(1-\tau) E_{i}[z]\right\}+(1-\omega) E_{i}[\theta]
$$

where $E_{i}\left[\bar{a}^{N}\right]=E_{i}[\theta]$ due to (4.58), and $E_{i}[z]=E_{i}[\theta]$ due to $z$ being unobservable to agent $i$. Thus, agent $i$ 's best response is just the conditional expectation of the state variable:

$$
a_{i}^{N d}=E_{i}[\theta]
$$

Representing (4.62) as a linear combination of observed signals:

$$
a_{i}^{N d}=\kappa^{N d} x_{i}+\left(1-\kappa^{N d}\right) y
$$

implies:

$$
\kappa^{N d}=\frac{\alpha}{\alpha+\beta}
$$

where $\kappa^{N d}$ and $1-\kappa^{N d}$ are Bayesian weights. Substituting (4.58) and (4.63) into (4.60), taking expectations of the resulting expression, and substituting (4.64) yields a deviating agent's expected payoff:

$$
E\left[u_{i}^{N d}\right]=-\frac{1}{\alpha+\beta}-\frac{\omega(1-\tau)^{2}}{\gamma}-p_{y} .
$$

Comparing $E\left[u_{i}^{N}\right]$ in (4.59) and $E\left[u_{i}^{N d}\right]$ in (4.65) reveals that a deviating agent suf- 
fers a smaller dispersion loss (first term in both expressions). Having access to two signals about $\theta$, a deviating agent is able to form a more accurate forecast of the average action (which is equal to $\theta$ ). Thus, a deviating agent suffers a smaller dispersion loss at the expense of having to incur an information fee. The variance of $\phi$ loss term is unaffected, as agent $i$ 's decision whether or not to purchase public information has no effect on agent $i$ 's inability to offset the noise $\phi$ in the $z$-component of the CB's rule.

Agent $i$ has no incentive to deviate from a non-purchasing population when the benefit of acquiring $y$ no longer outweighs the cost of doing so. Formally, agent $i$ 's optimal strategy with regard to purchasing $y$ is as follows:

$$
\begin{cases}\text { if } p_{y}<p_{\text {min }}, & \nu_{i}=1 \\ \text { if } p_{y}=p_{\text {min }}, & \nu_{i} \in\{0,1\} \\ \text { if } p_{\text {min }}<p_{y}, & \nu_{i}=0,\end{cases}
$$

where $p_{\min }$ is the critical value for which $E_{i}\left[u_{i}^{N}\right]=E_{i}\left[u_{i}^{N d}\right]$. Since we are interested in a symmetric NE in which no agent finds it worthwhile to purchase $y$, a N-NE exists for:

$$
p_{\min }<p_{y}
$$

where:

$$
p_{\min } \equiv \frac{\alpha}{(\alpha+\beta) \beta} .
$$

The fact that the derived solution for $p_{\min }$ is strictly positive implies that, for any combination of values of $\alpha, \beta, \omega$, and $\tau$, there always exists a subset of sufficiently low values of $p_{y}$ for which a N-NE does not exist. In other words, provided that information cost is sufficiently low, agent $i$ invariably finds it worthwhile to deviate from a non-purchasing population, acquire $y$, and, thereby, improve the accuracy of its forecast about $\theta$.

The decision about whether or not to acquire $y$ - decision to deviate $(N d)$ or not to deviate $(N)$ from a non-purchasing population, respectively - affects agent $i$ 's expected payoff only through the forecast error of $E_{i}[\theta]$ and information cost. Neither of the two terms depend on the payoff parameter $\omega$ nor the CB's choice of $\tau$, thus, the critical value $p_{\min }$ is also independent of $\omega$ and $\tau$. Suppose that $p_{y}=p_{\min }$ and agent $i$ is indifferent between purchasing and not purchasing $y$. A marginal increase in $\alpha$ would have no effect on $E_{i}\left[u_{i}^{N}\right]$, but would increase $E_{i}\left[u_{i}^{N d}\right]$, as any increase in the quality of $y$ also improves the accuracy of $E_{i}\left[\theta \mid x_{i}, y\right]$. Hence, agent $i$ would be willing to pay a higher price for deviating from a non-purchasing population, which 
implies a positive effect on $p_{\min }$. Since this is true regardless of the initial value of $\alpha$, the positive effect is monotonic, i.e. $p_{\min }$ is strictly increasing in $\alpha$. Meanwhile a marginal increase in $\beta$ would increase both $E_{i}\left[u_{i}^{N}\right]$ and $E_{i}\left[u_{i}^{N d}\right]$, as any increase in the quality of $x_{i}$ improves the accuracy of both $E_{i}\left[\theta \mid x_{i}\right]$ and $E_{i}\left[\theta \mid x_{i}, y\right]$. However, the marginal benefit from an increase in $\beta$ is stronger in the $N$-case ${ }^{36}$. Thus, a previously indifferent agent $i$ would no longer find it worthwhile to purchase $y$ leading to $p_{\min }$ to be strictly decreasing in $\beta$.

Comparing the two critical values reveals that:

$$
0<p_{\min }<p_{\max }
$$

for all parameter values and $0<\tau<1$. In the limit, as $\tau \rightarrow 0$, the two critical values coincide, as the rule collapses to just $z$ and optimal actions become equal to the corresponding expectations of the state variable: $a_{i}^{P}=a_{i}^{N d}=E_{i}\left[\theta \mid x_{i}, y\right]$ and $a_{i}^{P d}=$ $a_{i}^{N}=E_{i}\left[\theta \mid x_{i}\right]$. As Figure 4.8 illustrates, for $0<\tau<1$, there always exists a range of $p_{y}$ for which both types of NE exist. The size of the $\left(p_{\min }, p_{\max }\right)$ range depends on the setting of $\tau$. Namely, $p_{\min }$ remains fixed, whereas $p_{\max }$ is monotonically increasing in $\tau$, thereby widening the $\left(p_{\min }, p_{\max }\right)$ range when $\tau$ increases. In other words, by committing to a particular value of $\tau$, the $\mathrm{CB}$ is able to influence the value of $p_{\max }$. We expand on this idea and discuss other policy considerations in the following Section.

\subsubsection{Implications for policy design}

We maintain the assumption that the CB aims to minimise its loss function defined in (4.1). We divide our analysis of the optimal setting of $\tau$ based on how actual value of $p_{y}$ compares to critical values $p_{\min }$ and $\left.p_{\max }\right|_{\tau=1}$. We begin with the more

\footnotetext{
${ }^{36}$ The marginal benefit of an increase $\beta$ in the $N$-case is $\frac{\partial E_{i}\left[u_{i}^{N}\right]}{\partial \beta}=\frac{1}{\beta^{2}}$ and in the $N d$-case is $\frac{\partial E_{i}\left[u_{i}^{N d}\right]}{\partial \beta}=\frac{1}{(\alpha+\beta)^{2}}$.
}

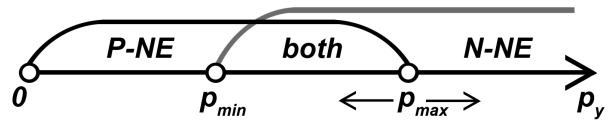

Figure 4.8: Public information acquisition. Existence of P-NE and N-NE 
straightforward cases in which only one type of $\mathrm{NE}$ exists $^{37}$ : only the P-NE exists for $0<p_{y} \leq p_{\min }$ and only the N-NE exists for $\left.p_{\max }\right|_{\tau=1} \leq p_{y}$. The discussion of these cases is presented in Section 4.3.4.1. We then turn to the subset of cases for which both NE types potentially exist: $p_{\min }<p_{y}<\left.p_{\max }\right|_{\tau=1}$. In Section 4.3.4.2, we identify a solution for $\tau$ under the working assumption that inducing the N-NE is desirable. We do so in order to identify a lower bound on the possible subset of values within which the optimal $\tau$ must lie. Finally, in Section 4.3.4.3, we investigate the validity of the initial assumption that a N-NE is desirable, and, in doing so, we refine our conclusions about the optimal setting of $\tau$ made in the previous Section.

\subsubsection{Cases in which only one type of NE exists}

The expression for CB's expected loss in a P-NE is analogous to that in the private information acquisition scenario and given by (4.37):

$$
E\left[L^{P}\right]=\frac{\alpha \tau^{2}}{[\alpha+\beta(1-\omega \tau)]^{2}}+(1-\tau)^{2} \cdot \frac{1}{\gamma}
$$

We already know that the CB's problem of minimising the P-NE expected loss in (4.37) has a unique interior solution $\tau=\tau_{y}^{P}$, such that $0<\tau_{y}^{P}<1 .{ }^{38,39}$ Since only the P-NE exists for $0<p_{y} \leq p_{\text {min }}$, over this range of actual price values it is optimal for the $\mathrm{CB}$ to commit to a rule characterised by $\tau=\tau_{y}^{P}$.

As far as the N-NE is concerned, setting $\kappa=\kappa^{N}=1$ in (4.36) yields:

$$
E\left[L^{N}\right]=\frac{(1-\tau)^{2}}{\gamma}
$$

The CB's expected loss in (4.68) is minimised for $\tau=\tau_{y}^{N}=1$. By committing to a rule that specifies the instrument adjustment to be equal to the market signal, the $\mathrm{CB}$ is able to take full advantage of the perfect market signal and perfectly align its instrument with the realised value of the fundamental: $g=\bar{a}^{N}=\theta$. Consequently, by ignoring its noisy private information, the $\mathrm{CB}$ is able to achieve zero expected loss:

$$
E\left[L^{N}\right]_{\tau=\tau_{y}^{N}}=0
$$

To identify the subset of price values for which only the N-NE exists, we need to

\footnotetext{
${ }^{37}$ For analytical convenience, we assume that only the P-NE exists at $p_{y}=p_{\min }$, even though agent $i$ is indifferent between deviating and not deviating from a non-purchasing population. Similarly, we assume that only the N-NE exists at $p_{y}=p_{\max }$.

${ }^{38}$ As noted in Section 4.2.3.5, the exact solution for $\tau_{y}^{P}$ is not tractable. However, the argument for the existence of a unique minimum presented in Appendix D.3 applies here as well.

${ }^{39}$ It should be noted that the implied value for $\tau_{y}^{P}$ here differs from $\tau^{P}$ in Section 4.2.3.5, since here $\tau_{y}^{P}$ is derived under the assumption of an exogenously fixed $\beta$.
} 
derive the upper price limit for the existence of the P-NE. Since $p_{\max }$ in (4.54) is strictly increasing in $\tau$, the upper limit on $p_{\max }$ corresponds to $\tau=1$ :

$$
\left.p_{\max }\right|_{\tau=1}=\frac{\alpha(\alpha+\beta)}{\beta[\alpha+\beta(1-\omega)]^{2}}
$$

Since the N-NE is the sole NE type that arises for $\left.p_{\max }\right|_{\tau=1} \leq p_{y}$, over this range of price values it is optimal for the CB to commit to $\tau=\tau_{y}^{N}=1$. Together with our conclusion about the $0<p_{y}<p_{\min }$ subset of prices, this result is illustrated by Figure 4.9 and constitutes Proposition 4.3.

Proposition 4.3: Given agents make a payoff-maximising decision about public information acquisition and the information cost is fixed at $p_{y}$, it is optimal for the central bank to commit to:

(i) $\tau=\tau_{y}^{P}$ for $0<p_{y} \leq p_{\text {min }}$, implying only the purchasing Nash equilibrium then exists, and;

(ii) $\tau=\tau_{y}^{N}=1$ for $\left.p_{\max }\right|_{\tau=1} \leq p_{y}$, implying only the non-purchasing Nash equilibrium then exists.

In the latter case, the reflection problem is eliminated, and the central bank is able to achieve the first-best outcome in terms of its own objective.

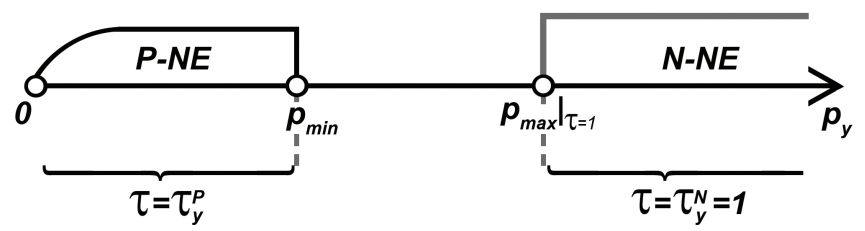

Figure 4.9: Public information acquisition. Optimal policy design for cases in which only the P-NE or the N-NE exists

Instrument adjustment of the form $g=\bar{a}^{N}$ in the N-NE implies that individual agents no longer need to match the noisy $z$ signal with their actions, as it is absent from the CB's rule. Thus, the variance of $\phi$ loss is eliminated from agent $i$ 's expected payoff in (4.59), and only the loss relating to the forecast error of $E_{i}\left[\theta \mid x_{i}\right]$ remains:

$$
E\left[u_{i}^{N}\right]_{\tau=\tau_{y}^{N}}=-\frac{1}{\beta}
$$

It should be noted that commitment to $\tau=\tau_{y}^{N}$ is both loss-minimising for the CB as well as payoff-maximising for individual agents. 


\subsubsection{Inducing the N-NE when both NE types exist}

We have characterised optimal policy design and NE outcomes for two price intervals: $0<p_{y} \leq p_{\min }$ and $\left.p_{\max }\right|_{\tau=1} \leq p_{y}$. Now we turn to determining CB's optimal strategy for the subset of cases for which it is possible for either type of NE to exist and that correspond to $p_{y} \in\left(p_{\min },\left.p_{\max }\right|_{\tau=1}\right)$. In this Section we focus on commitment strategies that induce the N-NE outcome and in the following Section we assess if inducing the N-NE is, in fact, optimal.

As already mentioned above, the $\mathrm{CB}$ is able to manipulate the value of $p_{\max }$ through its choice of $\tau$. Thus, for a given $p_{y}$, the CB is able to choose $\tau^{\prime}$ such that $\left.p_{\text {max }}\right|_{\tau=\tau^{\prime}} \leq p_{y}$ thereby effectively inducing the N-NE. Figure 4.10 demonstrates how the CB can induce the N-NE at $p_{y}$ by committing to $\tau^{\prime}$ such that $\left.p_{\max }\right|_{\tau=\tau^{\prime}}$ falls below $p_{y}$.

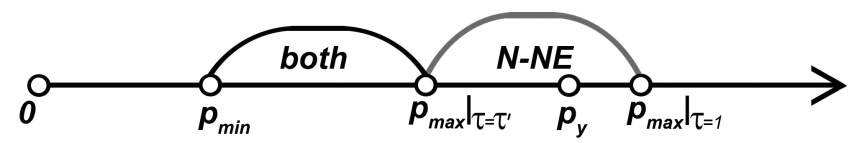

Figure 4.10: Public information acquisition. Inducing N-NE at given $p_{y}$ by setting $\tau=\tau^{\prime}$

Suppose for a moment that the CB aims to prevent the P-NE outcome from arising for any $p_{y} \in\left(p_{\min },\left.p_{\max }\right|_{\tau=1}\right)$. As mentioned before, the two critical price values $-p_{\min }$ and $p_{\max }-$ coincide for $\tau=0$. Thus, by committing to $\tau=0$ over the price range $p_{\min }<p_{y}<\left.p_{\max }\right|_{\tau=1}$, the CB can ensure that the N-NE is the sole NE type to arise. However, commitment to a rule of the form $g=z$ implies that the CB cannot take advantage of the perfect market signal, since the weight on $\bar{a}^{N}$ is zero. Hence, setting $\tau=0$ for $p_{y} \in\left(p_{\min },\left.p_{\max }\right|_{\tau=1}\right)$ is suboptimal.

Next we consider what is the optimal commitment strategy over the price range $p_{\min }<p_{y}<\left.p_{\max }\right|_{\tau=1}$, given the assumption that the CB aims to induce the N-NE. In this case the $\mathrm{CB}$ faces a constrained optimisation problem of the form:

$$
\min E\left[L^{N}\right]=\frac{(1-\tau)^{2}}{\gamma} \quad \text { s.t. } \quad p_{\max } \leq p_{y}
$$

where $E\left[L^{N}\right]$ is taken from (4.68). In order to prevent the possibility of the P-NE existing, the $\mathrm{CB}$ needs to choose a $\tau$ that corresponds to a value of $p_{\text {max }}$ that lies below the actual price $p_{y}$. At the same time, the chosen $\tau$ needs to achieve the 
lowest possible expected loss. The latter is strictly decreasing in $\tau$ for $0<\tau<1$, since any increase in $\tau$ means less weight is placed on the noisy signal $z$ and more weight is placed on the perfect market signal. Meanwhile, $p_{\max }-$ as given by (4.54) - is strictly increasing in $\tau$. Considering the direction of influence of $\tau$ on $E\left[L^{N}\right]$ and $p_{\max }$, it immediately follows that the constraint must be binding. Thus, under the assumption that the N-NE needs to be induced, the optimal value for $\tau$ is the solution to $p_{y}=p_{\max }:{ }^{40}$

$$
\tau_{y}^{*} \equiv \frac{1}{\beta \omega}\left[\alpha+\beta-\sqrt{\frac{\alpha(\alpha+\beta)}{\beta p_{y}}}\right] .
$$

Namely, the strategy of setting $\tau=\tau_{y}^{*}$ involves commitment to a value of $\tau$ below unity such that individual agents find it just not worthwhile to purchase $y$ at a given $p_{y}$. Commitment to a $\tau$ that lies above $\tau_{y}^{*}$, violates the constraint, thereby, not precluding the existence of the P-NE; while setting $\tau$ below $\tau_{y}^{*}$ leads to an expected outcome that can always be improved by committing to a higher $\tau$. Hence, $\tau=\tau_{y}^{*}$ is the optimal solution, assuming the need to induce the N-NE. It should be noted that, at $p_{y}=p_{\min }$, the solution value appropriately collapses to $\tau_{y}^{*}=0$, which leads to $p_{\min }=\left.p_{\max }\right|_{\tau=\tau_{y}^{*}}$.

By setting $\tau=\tau_{y}^{*}$ the $\mathrm{CB}$ is able to prevent the $\mathrm{P}-\mathrm{NE}$ from arising at a given $p_{y}$ by achieving $p_{y}=p_{\max }$. As a result, the $\mathrm{CB}$ is able to induce the N-NE while still being able to make use (albeit limited, since $\tau<1$ ) of the perfect market signal. The identified strategy constitutes Proposition 4.4.

Proposition 4.4: Given that the central bank aims to minimise its loss subject to inducing a non-purchasing Nash equilibrium, and the information cost $p_{y}$ lies within the interval $\left(p_{\min },\left.p_{\max }\right|_{\tau=1}\right)$, commitment to $\tau=\tau_{y}^{*}$ is the optimal strategy.

If the $\mathrm{CB}$ commits to $\tau=\tau_{y}^{*}$, the associated expected loss is derived by substituting (4.73) into (4.68):

$$
E\left[L^{N}\right]_{\tau=\tau_{y}^{*}}=\frac{1}{\gamma} \cdot\left\{1-\frac{1}{\beta \omega}\left[\alpha+\beta-\sqrt{\frac{\alpha(\alpha+\beta)}{\beta p_{y}}}\right]\right\}^{2} .
$$

It should be noted, that the closer the actual $p_{y}$ lies to $p_{\min }$ (and the further it lies from $\left.p_{\max }\right|_{\tau=1}$ ), the lower the associated optimal value $\tau_{y}^{*}$ is. In other words, in order to induce the N-NE at lower prices, the CB has to reduce the responsiveness

\footnotetext{
${ }^{40}$ Solving the equation yields a second root that is strictly greater than unity and, thus, not relevant.
} 
of its instrument to the perfect market signal, which leads to a greater expected loss.

There is a consideration worth noting in relation to the strategy of setting $\tau=\tau_{y}^{*}$ over the price range $p_{\min }<p_{y}<\left.p_{\max }\right|_{\tau=1}$. Commitment to $\tau=\tau_{y}^{*}$ with the aim of preventing the P-NE from arising is vulnerable to time-inconsistency. Namely, once individual agents decide to not acquire $y$ and take their actions based on commitment to $\tau=\tau_{y}^{*}$, the market signal reveals $\theta$ perfectly. This creates a strong incentive for the $\mathrm{CB}$ to renege on its commitment and ignore its private information in order to fully take advantage of the perfect signal about $\theta$. The incentive to set $\tau=1$ ex post makes the $\tau=\tau_{y}^{*}$ precommitment strategy time-inconsistent, thereby undermining the credibility of such commitment ${ }^{41}$.

As noted above, the optimality of the $\tau=\tau_{y}^{*}$ strategy is predicated on the assumption that it is optimal to induce the N-NE at prices for which both NE types exist if $\tau_{y}^{*}<\tau$. In the following Section, we consider whether the true optimum over the price range $p_{\min }<p_{y}<\left.p_{\max }\right|_{\tau=1}$ coincides with or exceeds $\tau_{y}^{*}$ identified in this Section.

\subsubsection{Global optimality when both NE types exist}

In this Section we discuss several points relevant to the optimal setting of the policy parameter $\tau$ when information cost $p_{y}$ lies in the interval $\left(p_{\min },\left.p_{\max }\right|_{\tau=1}\right)$. In the previous Section we discussed a commitment strategy $\left(\tau=\tau_{y}^{*}\right)$ that induces the $\mathrm{N}-\mathrm{NE}$ (and prevents the existence of P-NE). The question arises whether the CB would find it optimal to induce the N-NE for some price values and why.

To answer the latter question, it is important to note that the CB strictly prefers the N-NE outcome over the P-NE outcome for any given positive $\tau$, which is evident from comparing (4.37) and (4.68). This is not surprising, since the N-NE produces a market signal $\bar{a}^{N}$ that perfectly reveals the state variable, whereas in the P-NE the market signal $\bar{a}^{P}$ is a linear combination of $\theta$ and a noisy public signal. As a result, for a given $\tau$, the N-NE outcome is strictly superior in terms of CB's expected loss. In fact, from (4.37) and (4.68):

$$
E\left[L^{P}\right]=E\left[L^{N}\right]+\frac{\alpha \tau^{2}}{[\alpha+\beta(1-\omega \tau)]^{2}}
$$

On the one hand, this means that inducing the N-NE has the potential to be the globally optimal strategy. On the other hand, since commitment to $\tau=\tau_{y}^{*}<1$ does

\footnotetext{
${ }^{41}$ Same applies to the strategy of setting $\tau=0$ over the price range $p_{\min } \leq p_{y}<\left.p_{\max }\right|_{\tau=1}$.
} 
not achieve the first-best outcome of zero loss in the N-NE, there is potential for a globally optimal solution that exceeds $\tau_{y}^{*}$. Commitment to a value of $\tau$ that exceeds $\tau_{y}^{*}$ unambiguously improves the N-NE outcome, yet it also no longer precludes the $\mathrm{P}-\mathrm{NE}$ from arising, in which case the question is whether the P-NE outcome for the chosen $\tau$ is equal to or greater than the N-NE outcome for $\tau=\tau_{y}^{*}<1$. In what follows we explore both possibilities for a globally optimal strategy in more detail.

Firstly, we highlight a few properties of the CB's expected loss expressions in (4.37) and (4.68). Prior to substitution of $\tau$, the CB's two expected loss expressions $E\left[L^{P}\right]$ and $E\left[L^{N}\right]$ are independent of $p_{y}$. The latter determines the existence of the P-NE under commitment to a specific value of $\tau$. Figure 4.11 shows a plot of $E\left[L^{P}\right]$

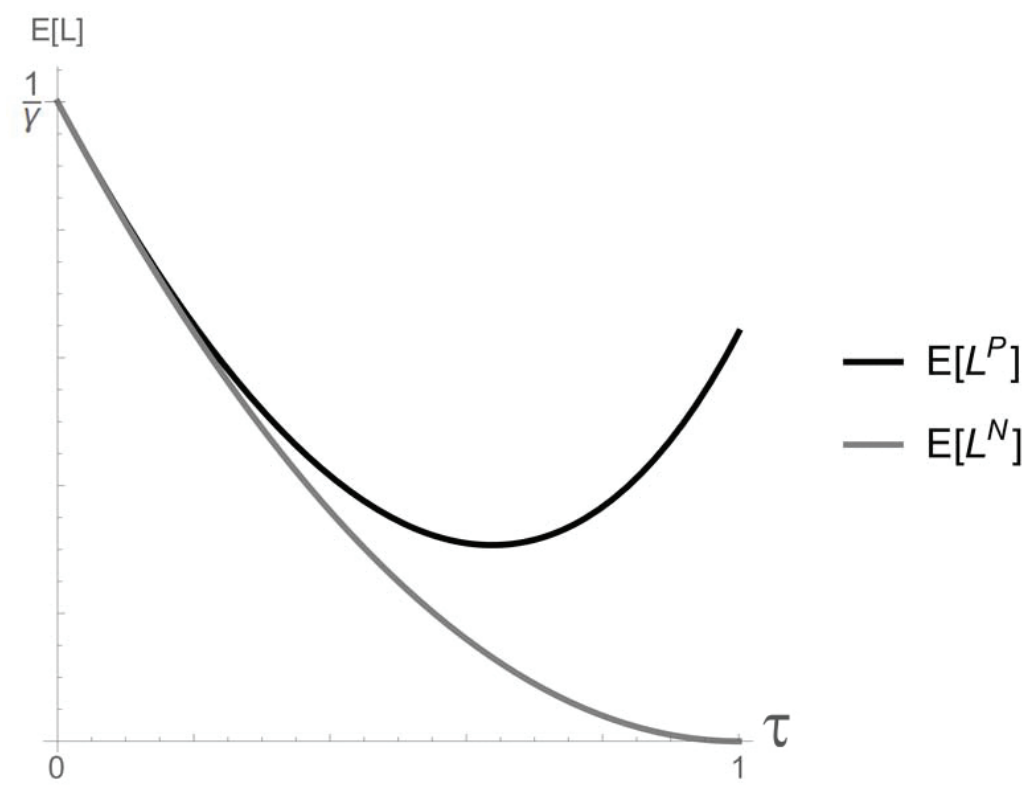

Figure 4.11: Public information acquisition. CB's expected loss in the P-NE and the N-NE as a function of $\tau$

and $E\left[L^{N}\right]$ as a function of $\tau$. In the limit, both expected losses are equal to the variance of the CB's private information $\frac{1}{\gamma}$, as $\tau$ approaches zero (as the rule approaches $g=z$ ). The N-NE expected loss is convex in $\tau$ and achieves its minimum (zero) at $\tau=\tau^{N}=1$. The P-NE expected loss is also convex in $\tau$, but achieves its minimum at $\tau=\tau^{P}$, where $\tau^{P}$ is a proper fraction.

Now we consider a specific scenario of commitment to a value of $\tau$ that exceeds $\tau_{y}^{*}$. Namely, we compare the option of inducing the N-NE as the sole NE type against allowing the existence of both NE types while achieving the first-best outcome in the N-NE. Suppose that, given $p_{y}$, the CB can either choose $\tau=\tau_{y}^{*}$ or $\tau=\tau_{y}^{N}=1$. Under the first option, only the N-NE exists and the CB's expected loss is given by 
(4.74):

$$
E\left[L^{N}\right]_{\tau=\tau_{y}^{*}}=\frac{1}{\gamma} \cdot\left\{1-\frac{1}{\beta \omega}\left[\alpha+\beta-\sqrt{\frac{\alpha(\alpha+\beta)}{\beta p_{y}}}\right]\right\}^{2} .
$$

Under the second option, if the N-NE arises, the CB's expected loss is zero, as show in (4.69):

$$
E\left[L^{N}\right]_{\tau=\tau_{y}^{N}}=0
$$

If, however, the P-NE arises, then the CB's expected loss is obtained by substituting $\tau=\tau_{y}^{N}=1$ into (4.37):

$$
E\left[L^{P}\right]_{\tau=\tau_{y}^{N}}=\frac{\alpha}{[\alpha+\beta(1-\omega)]^{2}}
$$

Since we have already established that the expected-loss minimising setting for $\tau$ in the $\mathrm{P}-\mathrm{NE}$ is a proper fraction $\left(0<\tau_{y}^{P}<1\right)$, by setting $\tau=\tau_{y}^{N}=1 \mathrm{CB}$ precludes the achievement of the first-best outcome in the P-NE.

\begin{tabular}{|l|c|c|}
\hline & P-NE & N-NE \\
\hline$\tau=\tau_{y}^{*}$ & - & $E\left[L^{N}\right]_{\tau=\tau_{y}^{*}}$ \\
$\tau=\tau_{y}^{N}=1$ & $E\left[L^{P}\right]_{\tau=\tau_{y}^{N}}$ & 0 \\
\hline
\end{tabular}

Table 4.1: Public information acquisition. Expected loss outcomes for $\tau=\tau_{y}^{*}$ and $\tau=\tau_{y}^{N}=1$.

Table 4.1 conveniently presents CB's expected outcomes, depending on the value of $\tau$ chosen and the NE type. It is clear that $\tau=\tau_{y}^{N}=1$ is the dominant strategy for $p_{\min }<p_{y}<\left.p_{\max }\right|_{\tau=1}$, if $E\left[L^{P}\right]_{\tau=\tau_{y}^{N}} \leq E\left[L^{N}\right]_{\tau=\tau_{y}^{*}}$. The latter condition is true for $0<\gamma<\gamma_{1}$, where:

$$
\begin{aligned}
\gamma_{1} & \equiv \frac{[\alpha+\beta(1-\omega)]^{2}\left(1-\tau_{y}^{*}\right)^{2}}{\alpha}= \\
& =\frac{[\alpha+\beta(1-\omega)]^{2}}{\beta^{3} \omega^{2}} \cdot\left\{\frac{\alpha+\beta}{p_{y}}-2 \beta[\alpha+\beta(1-\omega)] \sqrt{\frac{\alpha+\beta}{\alpha \beta p_{y}}}+\frac{\beta[\alpha+\beta(1-\omega)]^{2}}{\alpha}\right\}
\end{aligned}
$$

In other words, when the quality of CB's private information is sufficiently low, the P-NE outcome under $\tau=\tau_{y}^{N}=1$ is preferable to inducing the N-NE by setting $\tau=\tau_{y}^{*}$. This is because the loss term associated with the variance of $\phi$ in the N-NE outweighs the loss term associated with the noisiness of the market signal in the P-NE. As $p_{y}$ approaches $\left.p_{\max }\right|_{\tau=1}$, the N-NE-inducing $\tau_{y}^{*}$ value approaches unity, the variance of $\phi$ loss term approaches zero, and the critical value $\gamma_{1}$, appropriately, approaches to zero. Figure 4.12 demonstrates the two strategies. The top panel de- 

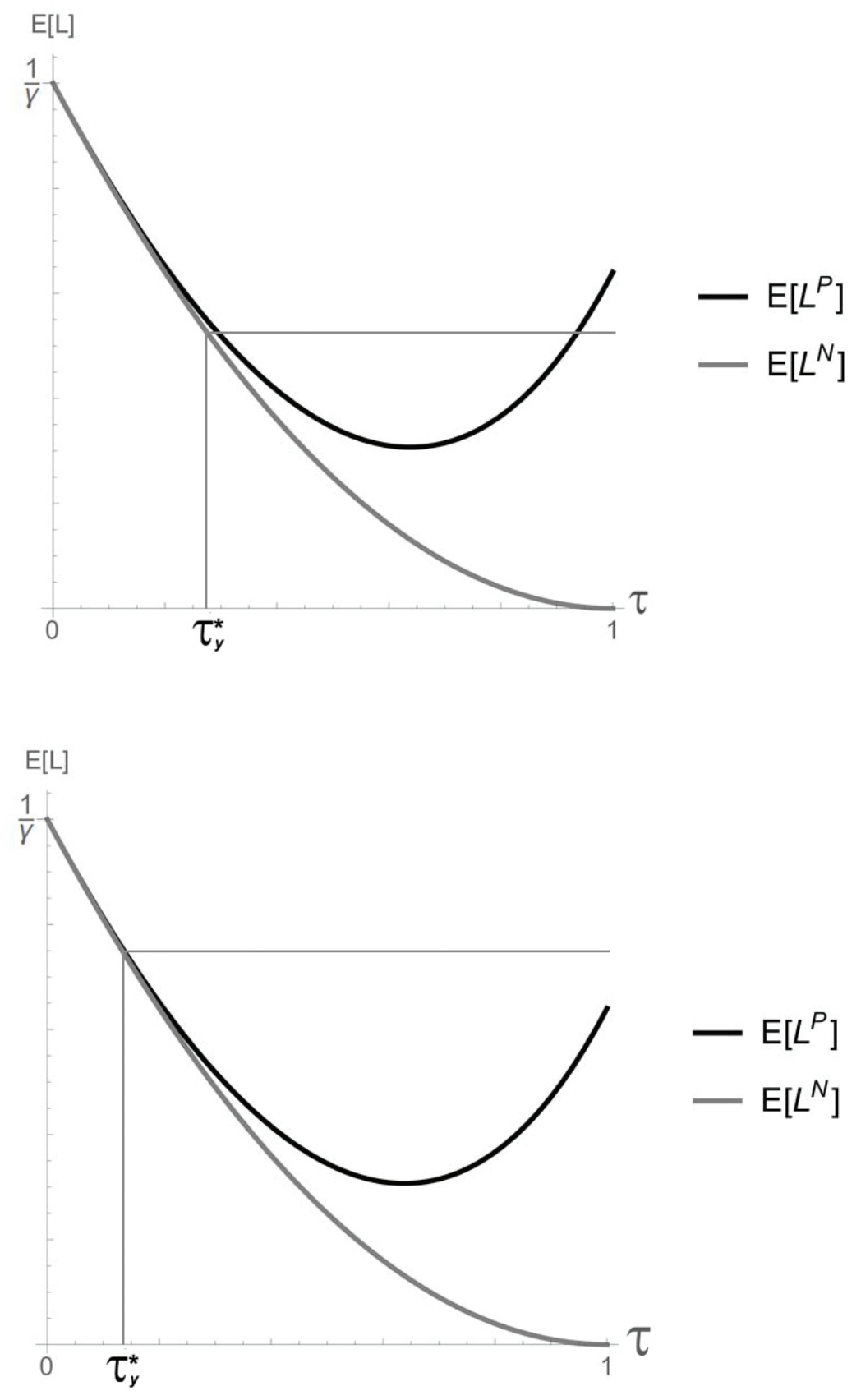

Figure 4.12: Public information acquisition. CB's expected loss in the P-NE and the N-NE and optimality of commitment to $\tau=1$

picts the case of $E\left[L^{N}\right]_{\tau=\tau_{y}^{*}}<E\left[L^{P}\right]_{\tau=\tau_{y}^{N}}$ when $\tau=\tau_{y}^{*}$ is the preferable strategy, and the bottom panel illustrates the case of $E\left[L^{P}\right]_{\tau=\tau_{y}^{N}}<E\left[L^{N}\right]_{\tau=\tau_{y}^{*}}$ when $\tau=\tau_{y}^{N}=1$ is the dominant strategy.

Next, we extend the above result and consider the conditions under which it might be optimal for the CB to commit to a value of $\tau$ that lies above $\tau=\tau_{y}^{*}$, yet below unity. In order to proceed, we evaluate the first derivative of $E\left[L^{P}\right]$ in (4.37) 

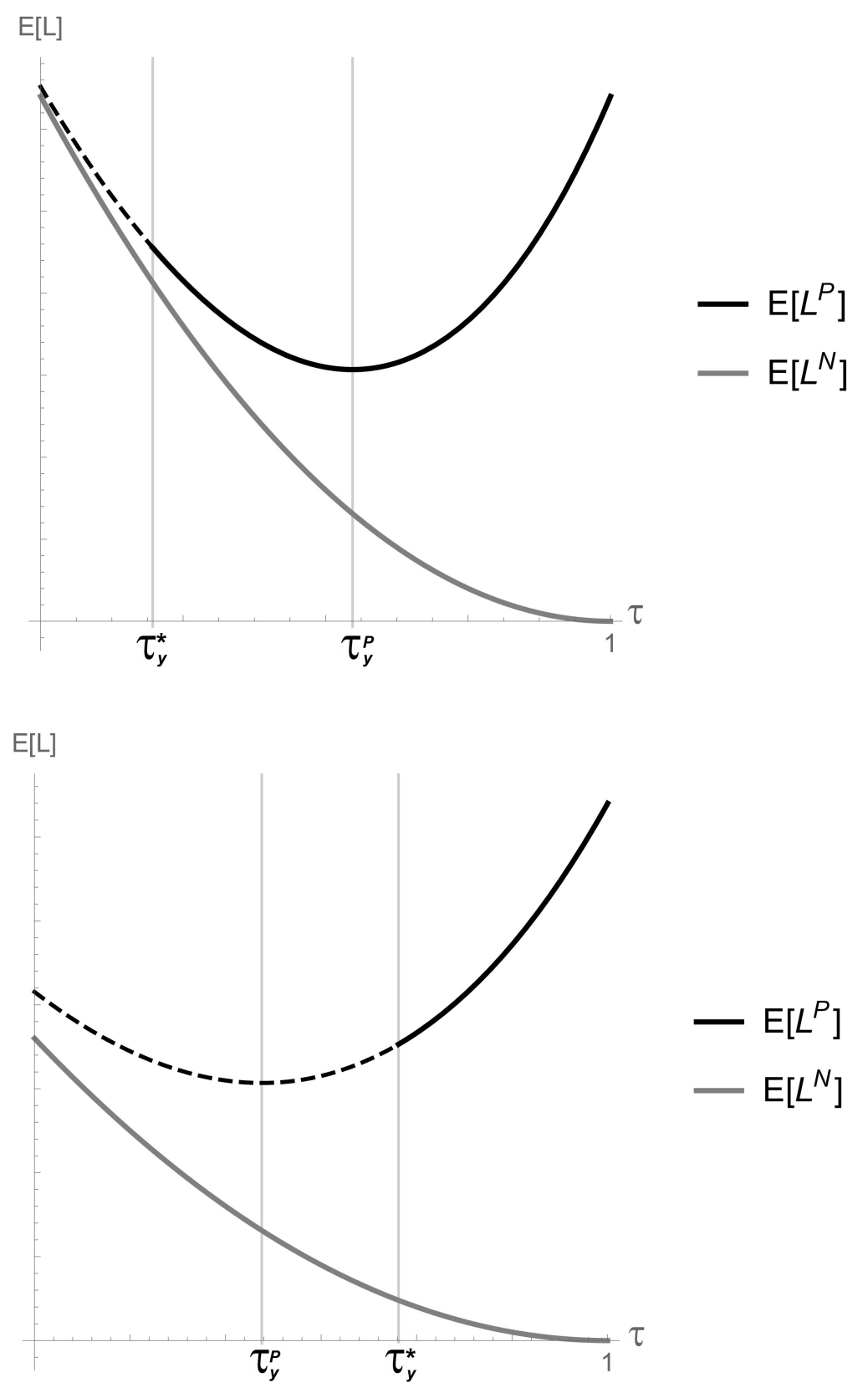

Figure 4.13: Public information acquisition. CB's expected loss in the P-NE and the N-NE and commitment strategies

with respect to $\tau$ at $\tau=\tau_{y}^{*}$ and look at what determines its sign. The sign of the derivative is negative when one of the following sets of conditions is satisfied:

$$
\left\{\begin{array}{l}
p_{\min }<p_{y}<\frac{\alpha}{\beta[\alpha+\beta(1-\omega)]} \\
0<\gamma<\Omega_{1}+\Omega_{2}
\end{array}\right.
$$


or

$$
\left\{\begin{array}{l}
\frac{\alpha}{\beta[\alpha+\beta(1-\omega)]} \leq p_{y}<\left.p_{\max }\right|_{\tau=1} \\
0<\gamma<\Omega_{1}-\Omega_{2}
\end{array}\right.
$$

where:

$$
\begin{aligned}
& \Omega_{1}=\frac{\alpha \omega}{\left[\beta^{2} p-\alpha(1-\beta p)\right] p} \\
& \Omega_{2}=\sqrt{\frac{\alpha(\alpha+\beta)\left[\alpha-\alpha \beta p-\beta^{2}(1-\omega) p\right]^{2}}{\beta\left[\beta^{2} p-\alpha(1-\beta p)\right]^{2} p}} .
\end{aligned}
$$

Alternatively, the sign of the derivative is positive when one of the following sets of conditions is satisfied:

$$
\left\{\begin{array}{l}
p_{\min }<p_{y}<\frac{\alpha}{\beta[\alpha+\beta(1-\omega)]} \\
\Omega_{1}+\Omega_{2}<\gamma
\end{array}\right.
$$

or

$$
\left\{\begin{array}{l}
\frac{\alpha}{\beta[\alpha+\beta(1-\omega)]} \leq p_{y}<\left.p_{\max }\right|_{\tau=1} \\
\Omega_{1}-\Omega_{2}<\gamma .
\end{array}\right.
$$

The straightforward implication of the sign of the derivative being negative at $\tau=\tau_{y}^{*}$ is that, for given $p_{y}, \tau_{y}^{*}$ lies below $\tau_{y}^{P}$. Similarly, a positive sign implies $\tau_{y}^{P}<\tau_{y}^{*}$. Figure 4.13 illustrates the two cases: (i) in the top panel, $E\left[L^{P}\right]$ is decreasing in $\tau$ at $\tau=\tau_{y}^{*}$, and (ii) in the bottom panel, $E\left[L^{P}\right]$ is increasing in $\tau$ at $\tau=\tau_{y}^{*}$.

The top panel in Figure 4.13 shows that, if $E\left[L^{P}\right]$ is decreasing in $\tau$ at $\tau=\tau_{y}^{*}$, increasing $\tau$ beyond $\tau_{y}^{*}$ results in the following. Firstly, both NE types exist instead of just N-NE. Secondly, both outcomes are improved initially. Thirdly, the P-NE outcome improves up to $\tau=\tau_{y}^{P}$, at which point the expected loss is minimised, and deteriorates beyond $\tau=\tau_{y}^{P}$. Finally, the N-NE outcome improves continuously as $\tau$ is increased. Employing the argument already used in the above scenario in which the $\mathrm{CB}$ is restricted to choosing between commitment to $\tau=\tau_{y}^{*}$ and $\tau=\tau_{y}^{N}=1$, it is possible to make further inference about commitment to $\tau=\tau_{y}^{P}$. Namely, by comparing $E\left[L^{N}\right]_{\tau=\tau_{y}^{*}}$ and $E\left[L^{P}\right]_{\tau=\tau_{y}^{P}}$ we can identify a set of conditions under which the strategy of committing to $\tau=\tau_{y}^{*}$ is strictly dominated by the strategy of committing to $\tau=\tau_{y}^{P}$. However, we are prevented from performing this exercise by the intractability of the $\tau_{y}^{P}$ value. We proceed to the bottom panel in Figure 4.13 
that illustrates the case when $\tau_{y}^{P}<\tau_{y}^{*}$. Increasing $\tau$ above $\tau_{y}^{*}$ improves the N-NE outcome, yet has a detrimental effect on the P-NE outcome, which precludes further analysis.

As demonstrated above, one obvious complication of increasing $\tau$ beyond $\tau_{y}^{*}$ relates to the fact that the $\mathrm{N}-\mathrm{NE}$ ceases to be the only potential type of NE. Our model does not produce a prediction on which NE type is more or less likely to occur, thus, preventing us from identifying a strictly dominant strategy with a globally optimal commitment solution. Hence, in order to proceed with the analysis of optimal policy design beyond the observations already made we would require to make further assumptions about the model.

\subsection{Concluding remarks}

In the present Chapter we examine two variations of the Morris and Shin (2018) model. In the first part of the Chapter, we introduce private information acquisition using the linear-cost approach from Colombo and Femminis (2008). In the second part of the Chapter, we consider public information acquisition with a flat-fee cost structure. In both scenarios we look at the interaction between monetary policy and individual agents' strategies for information use and acquisition. We then discuss implications that monetary policy design has for signal value of the market aggregate, in particular, the reflection problem.

The original Morris-Shin model as well as the variations analysed in this Chapter feature individual payoff functions that do not include an explicit coordination term, yet the motive to coordinate actions arises endogenously. We begin our discussion of the private information acquisition scenario by constructing a benchmark with no strategic complementarities in actions. This exercise allows us to highlight the way in which coordination affects individual actions and information acquisition strategies in the main model in this part of the Chapter. We define a measure of accuracy of CB's information about the state variable, which is implicit to the Morris and Shin (2018) discussion, and that helps us characterise the reflection problem. We perform a separate analysis of the purchasing equilibria, in which agents choose to purchase private information of above-zero quality, and the non-purchasing equilibria, in which agents do not find it optimal to acquire private signals.

We find that the reflection problem, indeed, arises when agents choose to purchase heterogeneous private information. However, direct comparison of our results and the original conclusions by Morris and Shin is precluded by the endogeneity of 
the quality of private information, which is an exogenous parameter in the Morris and Shin model, as well as the presence of an information cost parameter that is absent from the original model. Our analysis of the purchasing equilibria is further impeded by the intractability of the solution to the CB's optimisation problem. Nevertheless, we are able to ascertain that the CB finds it optimal to commit to a rule characterised by a policy parameter value that lies strictly above zero and below unity. We identify the conditions for the existence of purchasing equilibria with optimal commitment, taking the policy parameter value as given. We find that, for certain parameter values, the CB has the ability to induce a preferred type of equilibrium - purchasing or non-purchasing - by adjusting its policy rule accordingly. We conclude our discussion of purchasing equilibria by presenting a numerical example that is directly comparable with the example used in Morris and Shin (2018). Numerical parameter values are selected so that, at the point of equilibrium, the quality of private information is the same in both cases. The example shows that, in the presence of information acquisition, the $\mathrm{CB}$ commits to putting less weight on the market signal, which leads to individual agents putting more weight on their private signals and the CB suffering a greater expected loss.

In contrast to the purchasing equilibria, we are able to fully characterise the non-purchasing equilibrium solution. In the absence of heterogeneous private information acquisition, the reflection problem does not arise due to the signal value of the market aggregate being independent of the policy parameter. We find that it is optimal for the CB to commit to a rule that weighs the market signal and the CB's private signal proportionally to the signals' quality. Optimal commitment strategy minimises the CB's expected loss, yet does not maximise agents' individual expected payoffs. Although we are unable to directly compare outcomes in the purchasing and non-purchasing equilibria due to the intractability of the policy parameter solution in the purchasing case, we successfully identify a numerical example that proves that the set of parameter value combinations for which both equilibrium types exist is not empty.

In the second part of the Chapter we revert to the original Morris-Shin assumption of exogenous quality of agents' private information and introduce a flat fee for acquisition of public information. This scenario is of interest because public information acquisition is undesirable from the point of view of the CB, yet has a perceived benefit for individual agents. We characterise the purchasing and nonpurchasing equilibria separately and identify the conditions for the existence of each equilibrium type. We find that there always exists a set of information price values for which both types of equilibrium exist. Within this set of price values, the CB is 
able to induce the non-purchasing equilibrium as the sole equilibrium type through commitment to a policy rule that is designed with that aim in mind.

When only purchasing equilibrium exists, the optimal commitment rule is characterised by an intractable solution, however, that solution coincides with that implied in the Morris and Shin (2018) discussion. When only the non-purchasing equilibrium exists, it is optimal for the $\mathrm{CB}$ to ignore its private information and take full advantage of the perfect market signal. In this case, optimal commitment is both loss-minimising for the $\mathrm{CB}$ and payoff-maximising for individual agents. The $\mathrm{CB}$ is able to achieve the first-best outcome. In the final Section, we present additional inferences about optimal commitment strategies for the cases in which both equilibrium types exist. We find that our model specification prevents us from identifying a globally optimal solution. Potential additional assumptions can, for instance, allow for the assessment of each equilibrium type for focal-point properties and result in identifiable equilibrium ranking. Extending the analysis in this direction would allow further characterisation of optimal policy design.

Another promising direction in case of both model variations is to extend the analysis to include an efficient benchmark scenario. As mentioned in Section 4.2.1, constructing an efficient benchmark would enable the derivation of a collectively efficient degree of coordination. Comparing the latter with the equilibrium degree of coordination would provide additional insights into the inefficiencies associated with the use and acquisition of information as well as the reflection problem. However, the construction of an efficient benchmark requires additional assumptions, including the identification of a social welfare function, which may or may not coincide with the CB's objective function. 


\section{Conclusion}

There exists an established consensus in the central-banking community that central bank communication is an important element of monetary policy. Desirability of transparency and its impact on policy effectiveness has been the subject of a considerable body of theoretical work. The principal aim of this thesis is to contribute to the ongoing discussion of the inherent connection between central bank transparency, private sector knowledge, and effectiveness of policy.

In our analysis of optimal policy design, we focus on a game-theoretic approach that closely follows the seminal work by Morris and Shin $(2002,2005)$, while also borrowing key elements from James and Lawler (2011), Angeletos and Pavan (2007a), Hahn (2012), Colombo and Femminis (2008), and others. We construct and solve a number of models that are variations of what we refer to as the Morris and Shin framework. The distinguishing features of the scenarios that we analyse are defined in terms of the key assumptions that we make about the information structure of the economy. Other modelling assumptions that we vary across model variations include the form of payoff and objective functions, as well as monetary policy regimes.

Chapter 1 provides context and motivation for the modelling approach we employ in subsequent Chapters. We give an overview of practical considerations in relation to central bank communication as well as establishing the main points of contact with the theoretical literature on transparency and the Morris and Shin framework, in particular. We discuss the Morris and Shin model and some of its most prominent variations in considerable detail in order to lay the groundwork for our investigation in Chapters 2, 3, and 4. The review of this particular strand of literature demonstrates the key strengths of the Morris and Shin framework - its tractability and versatility. Chapter 1 also includes brief discussions of a number of topics that serve as building blocks for constructing the different scenarios that we use in our analysis of optimal policy design.

There are four prominent results from the social value of information literature that act as unifying themes and are of particular relevance to our investigation. 
Firstly, Morris and Shin (2002) find that, in a beauty-contest scenario, increasing central bank transparency can be detrimental to welfare, in particular if the underlying quality of public announcements is low. Secondly, James and Lawler (2011) report that full opacity in combination with an optimally designed rule achieves the first-best welfare outcome. Chapters 2 and 3 demonstrate the parallels between the two aforementioned results and our own findings. Thirdly, Colombo and Femminis (2008) and Colombo et al. (2014) establish that substitutability between the quality of public and private information arises in a variety of scenarios with costly private information acquisition. We confirm this relationship for the model variations presented in Chapters 3 and 4. Finally, Angeletos and Pavan (2007a) characterise informational inefficiencies that arise from the misalignment between equilibrium and socially optimal degrees of coordination. This idea is equally useful in our discussions of scenarios in all three Chapters.

In Chapter 2 we extend the seminal Morris and Shin (2002) model to include information stickiness in the private sector, central bank transparency, and direct policy intervention. We follow Hahn $(2010,2012)$ in assuming that a given proportion of population does not act upon new information, while the remainder of the population takes all available information into account; and, thus, distinguish between passive and active agents, respectively. The way instrument adjustment enters into this model variation, is a modelling element borrowed from James and Lawler (2011). In considering monetary policy design we compare two policy regimes - commitment and discretion. We also construct a benchmark scenario, which is characterised by efficient use of information by all economic agents, and use it to evaluate policy effectiveness. We find that in our model (i) commitment performs at least as well as discretionary policy, and (ii) optimally designed policy fails to eliminate informational inefficiencies that stem from information stickiness.

In Chapter 3 we extend the Morris and Shin (2002) model to include costly private information acquisition, central bank transparency, and direct policy intervention. As in Chapter 2, we follow James and Lawler (2011) in our assumptions about instrument adjustment. We distinguish between fixed and linear information cost scenarios. In the latter case, we implement the information cost structure from Colombo and Femminis (2008). Our findings are as follows: (i) in an economy with a fixed information cost, the combination of full opacity and an optimally designed policy rule achieves efficiency; (ii) whereas, in an economy with a linear-cost structure, optimal policy either eliminates or at least mitigates informational inefficiencies. 
In Chapter 4 we extend the Morris and Shin (2018) market signal model to include costly information acquisition. We separately consider private and public information acquisition. In the former case, we use a linear cost structure, whereas, in the latter case, we implement the fixed-fee assumption. In this Chapter, instead of evaluating policy performance in terms of social welfare and efficiency, we focus on the reflection problem. Namely, we assume that private sector actions have information value for the central bank and discuss how this assumption affects the bank's optimal commitment strategies. We identify the conditions for the existence of different types of equilibria and characterise the role of monetary policy in shaping said conditions.

This thesis succeeds in motivating the idea that the nature of the interaction between central bank communication and monetary policy is sensitive to the assumptions made about the information structure of the economy. There is undeniably considerable scope for further theoretical investigations into monetary policy effectiveness within the intersection between the fields of monetary and information economics. 


\section{Appendix A}

\section{A.1 Summary table of extensions and variations of the Morris and Shin framework}

\begin{tabular}{|c|c|c|}
\hline Source & Extension/Variation & Key Result \\
\hline $\begin{array}{l}\text { Morris and } \\
\text { Shin (2002) }\end{array}$ & Original model & $\begin{array}{l}\text { Increased disclosure can be } \\
\text { detrimental. }\end{array}$ \\
\hline $\begin{array}{l}\text { Morris and } \\
\text { Shin (2002) }\end{array}$ & $\begin{array}{l}\text { Social planner chooses opti- } \\
\text { mal quality of public infor- } \\
\text { mation; increasing the qual- } \\
\text { ity of public information is } \\
\text { costly. }\end{array}$ & $\begin{array}{l}\text { Zero disclosure is a common } \\
\text { solution. }\end{array}$ \\
\hline $\begin{array}{l}\text { Morris and } \\
\text { Shin }(2002)\end{array}$ & $\begin{array}{l}\text { Social planner chooses op- } \\
\text { timal quality of public in- } \\
\text { formation; quality of pub- } \\
\text { lic information has an upper } \\
\text { limit. }\end{array}$ & $\begin{array}{l}\text { "Bang-bang" solution: ei- } \\
\text { ther zero disclosure or max- } \\
\text { imum feasible quality of } \\
\text { public information. }\end{array}$ \\
\hline $\begin{array}{l}\text { Morris and } \\
\text { Shin (2002) }\end{array}$ & $\begin{array}{l}\text { Alternative payoff function: } \\
\text { actions of individual agents } \\
\text { are strategic substitutes. }\end{array}$ & $\begin{array}{l}\text { Agents overreact to private } \\
\text { information. }\end{array}$ \\
\hline $\begin{array}{l}\text { Morris and } \\
\text { Shin }(2002)\end{array}$ & $\begin{array}{l}\text { Alternative welfare func- } \\
\text { tion: deviation of the aver- } \\
\text { age action from fundamen- } \\
\text { tals. }\end{array}$ & $\begin{array}{l}\text { Overreaction to public in- } \\
\text { formation causes greater } \\
\text { welfare loss. }\end{array}$ \\
\hline $\begin{array}{l}\text { Morris and } \\
\text { Shin (2002), } \\
\text { Appendix }\end{array}$ & $\begin{array}{l}2 \text { agents in the private sec- } \\
\text { tor. Fundamentals variable } \\
\text { has two states. }\end{array}$ & $\begin{array}{l}\text { Better public information } \\
\text { can be detrimental. }\end{array}$ \\
\hline
\end{tabular}




\begin{tabular}{|c|c|c|}
\hline Source & Extension/Variation & Key Result \\
\hline $\begin{array}{l}\text { Morris and } \\
\text { Shin (2002), } \\
\text { Appendix }\end{array}$ & $\begin{array}{l}\text { More general payoff func- } \\
\text { tion. }\end{array}$ & $\begin{array}{l}\text { Better public information } \\
\text { can be detrimental. }\end{array}$ \\
\hline $\begin{array}{l}\text { Morris and } \\
\text { Shin (2002), } \\
\text { Appendix }\end{array}$ & $\begin{array}{l}\text { Alternative payoff function } \\
\text { with an externality contin- } \\
\text { gent on the average action. }\end{array}$ & $\begin{array}{l}\text { Better public information } \\
\text { can be detrimental even in } \\
\text { absence of private informa- } \\
\text { tion. }\end{array}$ \\
\hline $\begin{array}{l}\text { Morris and } \\
\text { Shin (2002), } \\
\text { Appendix }\end{array}$ & $\begin{array}{l}2 \text { agents in the private sec- } \\
\text { tor. The noise terms in } \\
\text { the private information of } \\
\text { agents are correlated. Bet- } \\
\text { ter public information can } \\
\text { be detrimental. }\end{array}$ & \\
\hline $\begin{array}{l}\text { Hellwig } \\
(2005)\end{array}$ & $\begin{array}{l}\text { Microfounded model of mo- } \\
\text { nopolistic competition. }\end{array}$ & $\begin{array}{l}\text { Greater transparency is } \\
\text { beneficial. Higher quality of } \\
\text { agents' private information } \\
\text { can be detrimental. }\end{array}$ \\
\hline $\begin{array}{l}\text { Morris and } \\
\text { Shin (2005) }\end{array}$ & $\begin{array}{l}\text { Dynamic setting. Public in- } \\
\text { formation is endogenous. }\end{array}$ & $\begin{array}{l}\text { Under transparency the } \\
\text { central bank is less in- } \\
\text { formed. }\end{array}$ \\
\hline $\begin{array}{l}\text { Morris et al. } \\
(2006)\end{array}$ & $\begin{array}{l}2 \text { agents in the private sec- } \\
\text { tor. The noise terms in } \\
\text { the private information of } \\
\text { agents and the central bank } \\
\text { are correlated. }\end{array}$ & $\begin{array}{l}\text { Disclosing information with } \\
\text { a correlated noise can be } \\
\text { detrimental. }\end{array}$ \\
\hline $\begin{array}{l}\text { Angeletos } \\
\text { and Pavan } \\
(2007 \mathrm{~b})\end{array}$ & $\begin{array}{l}\text { More general model: pay- } \\
\text { off function with an exter- } \\
\text { nality contingent on the av- } \\
\text { erage action. }\end{array}$ & $\begin{array}{l}\text { If equilibrium and socially } \\
\text { efficient degrees of coordina- } \\
\text { tion are not equal overreac- } \\
\text { tion to public/private infor- } \\
\text { mation is detrimental. }\end{array}$ \\
\hline $\begin{array}{l}\text { Angeletos } \\
\text { and Pavan } \\
(2007 \mathrm{~b})\end{array}$ & $\begin{array}{l}\text { More general model: payoff } \\
\text { function with an external- } \\
\text { ity contingent on the aver- } \\
\text { age action. Pigouvian taxes } \\
\text { contingent on the average } \\
\text { action. }\end{array}$ & $\begin{array}{l}\text { Optimal tax rate eliminates } \\
\text { inefficiency. }\end{array}$ \\
\hline
\end{tabular}




\begin{tabular}{|c|c|c|}
\hline Source & Extension/Variation & Key Result \\
\hline $\begin{array}{l}\text { Cornand and } \\
\text { Heinemann } \\
(2008)\end{array}$ & $\begin{array}{l}\text { Public information is dis- } \\
\text { closed to a proportion of the } \\
\text { private sector. Degree of } \\
\text { publicity chosen by the cen- } \\
\text { tral bank. }\end{array}$ & $\begin{array}{l}\text { Partial disclosure can be op- } \\
\text { timal. }\end{array}$ \\
\hline $\begin{array}{l}\text { Cornand and } \\
\text { Heinemann } \\
(2008)\end{array}$ & $\begin{array}{l}\text { Degree of publicity chosen } \\
\text { by the central bank. Public } \\
\text { information is interpreted } \\
\text { with individual noise. }\end{array}$ & $\begin{array}{l}\text { Lower degree of publicity } \\
\text { if interpretation is accurate. } \\
\text { Partial disclosure can be op- } \\
\text { timal. }\end{array}$ \\
\hline $\begin{array}{l}\text { James and } \\
\text { Lawler }(2011)\end{array}$ & $\begin{array}{l}\text { Central bank influences fun- } \\
\text { damentals via rule and } \\
\text { chooses optimal quality of } \\
\text { public information. }\end{array}$ & $\begin{array}{l}\text { Optimal rule and zero dis- } \\
\text { closure achieve efficiency. }\end{array}$ \\
\hline $\begin{array}{l}\text { James and } \\
\text { Lawler }(2011)\end{array}$ & $\begin{array}{l}\text { Alternative payoff function: } \\
\text { equilibrium degree of coor- } \\
\text { dination is smaller than so- } \\
\text { cially efficient. }\end{array}$ & $\begin{array}{l}\text { Increased disclosure is ben- } \\
\text { eficial. Increased quality of } \\
\text { agents' private information } \\
\text { can be detrimental. Opti- } \\
\text { mal rule achieves efficiency. }\end{array}$ \\
\hline $\begin{array}{l}\text { James and } \\
\text { Lawler } \\
(2012 b)\end{array}$ & $\begin{array}{l}\text { Alternative payoff function: } \\
\text { equilibrium degree of coor- } \\
\text { dination is smaller than so- } \\
\text { cially efficient. Public infor- } \\
\text { mation is disclosed to a pro- } \\
\text { portion of the private sec- } \\
\text { tor. Degree of publicity cho- } \\
\text { sen by the central bank. }\end{array}$ & Full disclosure is optimal. \\
\hline $\begin{array}{l}\text { James and } \\
\text { Lawler } \\
(2012 b)\end{array}$ & $\begin{array}{l}\text { Alternative payoff function: } \\
\text { equilibrium degree of coor- } \\
\text { dination is smaller than so- } \\
\text { cially efficient. Degree of } \\
\text { publicity chosen by the cen- } \\
\text { tral bank. Central bank } \\
\text { influences fundamentals via } \\
\text { rule. }\end{array}$ & $\begin{array}{l}\text { Optimal rule and zero dis- } \\
\text { closure achieve efficiency. }\end{array}$ \\
\hline
\end{tabular}




\begin{tabular}{|c|c|c|}
\hline Source & Extension/Variation & Key Result \\
\hline $\begin{array}{l}\text { James and } \\
\text { Lawler } \\
(2012 b)\end{array}$ & $\begin{array}{l}\text { Alternative payoff function: } \\
\text { equilibrium degree of coor- } \\
\text { dination is smaller than so- } \\
\text { cially efficient. Degree of } \\
\text { publicity chosen by the cen- } \\
\text { tral bank. Central bank } \\
\text { influences fundamentals via } \\
\text { rule. Instrument-setting re- } \\
\text { vealed at the offset. }\end{array}$ & $\begin{array}{l}\text { Zero and partial disclosure } \\
\text { is superior to full disclosure. } \\
\text { Numerical simulations show } \\
\text { that zero disclosure is supe- } \\
\text { rior to partial disclosure. }\end{array}$ \\
\hline $\begin{array}{l}\text { James and } \\
\text { Lawler } \\
(2012 \mathrm{a})\end{array}$ & $\begin{array}{l}\text { Heterogeneous quality of } \\
\text { private information in the } \\
\text { private sector (two types of } \\
\text { agents). Degree of publicity } \\
\text { chosen by the central bank. }\end{array}$ & $\begin{array}{l}\text { Better public information } \\
\text { can be detrimental. Full } \\
\text { disclosure is more likely to } \\
\text { be optimal, as compared to } \\
\text { the one agent type case. }\end{array}$ \\
\hline $\begin{array}{ll}\text { James and } \\
\text { Lawler } \\
(2012 \mathrm{a})\end{array}$ & $\begin{array}{l}\text { Heterogeneous quality of } \\
\text { private information in the } \\
\text { private sector (two types of } \\
\text { agents). Degree of publicity } \\
\text { chosen by the central bank. } \\
\text { Pigouvian taxes contingent } \\
\text { on the average action. }\end{array}$ & $\begin{array}{l}\text { Optimal tax rate eliminates } \\
\text { inefficiency. Optimal tax } \\
\text { scheme design unaffected by } \\
\text { the existence of two agent } \\
\text { types. }\end{array}$ \\
\hline $\begin{array}{l}\text { James and } \\
\text { Lawler } \\
(2012 \mathrm{a})\end{array}$ & $\begin{array}{l}\text { Heterogeneous quality of } \\
\text { private information in the } \\
\text { private sector (two types of } \\
\text { agents). Degree of publicity } \\
\text { chosen by the central bank. } \\
\text { Central bank influences fun- } \\
\text { damentals via rule. }\end{array}$ & $\begin{array}{l}\text { Zero, partial or full disclo- } \\
\text { sure are optimal depend- } \\
\text { ing on the parameter val- } \\
\text { ues. Optimal rule and opti- } \\
\text { mal degree of publicity can- } \\
\text { not achieve efficiency. }\end{array}$ \\
\hline
\end{tabular}

Table A.1: Summary of extensions and variations of the Morris and Shin framework 


\section{Appendix B}

\section{B.1 Hybrid payoff function: derivation of degrees of coordination}

Angeletos and Pavan (2007a) define the equilibrium degree of coordination as the 'slope of the best response with respect to aggregate activity' in equilibrium. Thus, to find the equilibrium degree of coordination for the hybrid payoff function from (2.8):

$$
u_{i}=-[1-(1-\lambda) r]\left[a_{i}-(1-\lambda r)(\theta+g)-\lambda r \bar{a}\right]^{2}-(1-\lambda) r\left(L_{i}-\bar{L}\right) .
$$

we first need to find the equilibrium action of an individual active agent. For this purpose we differentiate the payoff function with respect to $a_{i}$ and solve the first derivative for $a_{i}$ :

$$
a_{i}=\left[1-r+(1-\lambda) \lambda r^{2}\right](\theta+g)+r[1-(1-\lambda) \lambda r] \bar{a} .
$$

It is apparent from the coefficient on the average action $\bar{a}$ in equation (B.1) that the equilibrium degree of coordination is given by:

$$
E D o C=[1-(1-\lambda) \lambda r] r
$$

which is equal to $r$ for $\lambda \in\{0,1\}$. This is consistent with the characteristics of the M-S and J-L payoff functions (James and Lawler, 2011).

Next we look at the socially efficient benchmark case, where the CB is able to force individual agents to use their information in a collectively efficient way. In this case the 'slope of the best response with respect to aggregate activity in this fictitious game' is the collectively efficient degree of coordination (Angeletos and Pavan, 2007a). We begin by deriving the expression for social welfare by aggregating 
and normalising equation (2.8):

$$
W=\frac{1}{[1-(1-\lambda) r]} \int_{0}^{1} u_{i} d i
$$

Given that $\bar{L}$ is defined as:

$$
\bar{L}=\int_{0}^{1} L_{i} d i
$$

expression (B.3) becomes:

$$
W=-\int_{0}^{1}\left[a_{i}-(1-\lambda r)(\theta+g)-\lambda r \bar{a}\right]^{2} d i
$$

Before taking expectations of (B.4) we perform a series of substitutions, using simplified assumptions from Section 2.2. Firstly, we assume that individual action is a linear function of two signals (see (2.25)):

$$
a_{i}=\kappa_{1} x_{i}+\kappa_{2} z
$$

where the signals are defined as follows (see (2.22) and (2.29)):

$$
\begin{aligned}
x_{i} & =\theta+\varepsilon_{i} \\
z & =\theta+\phi .
\end{aligned}
$$

Secondly, assuming that $\varepsilon_{i}$ has a zero mean, the average action is given by (see $(2.4))$ :

$$
\bar{a}=\int_{0}^{1} a_{j} d j=\kappa_{1} \theta+\kappa_{2} z .
$$

Thirdly, since the CB is assumed to be able to set $\kappa_{1}, \kappa_{2}$ and $g$ simultaneously, it can be shown that the precise value of the instrument adjustment $g$ is irrelevant for this derivation exercise. Thus, we set $g=0$ and obtain the following welfare expression:

$$
W=-\int_{0}^{1}\left\{\kappa_{1}\left(\theta+\varepsilon_{i}\right)+\kappa_{2}(\theta+\phi)-(1-\lambda r) \theta-\lambda r\left[\kappa_{1} \theta+\kappa_{2}(\theta+\phi)\right]\right\}^{2} d i
$$

We proceed by taking unconditional expectations of (B.6), using the distributions of $\varepsilon_{i}, \theta$ and $\phi$ as assumed in Section 2.2. The resulting expression for expected welfare is given by:

$$
E[W]=-\left\{\left(1-\kappa_{1}-\kappa_{2}\right)^{2}(1-\lambda r)^{2} \sigma_{\theta}^{2}+\kappa_{1}^{2} \sigma_{\varepsilon}^{2}+(1-\lambda r)^{2} \kappa_{2} \sigma_{\phi}^{2}\right\} .
$$


The expected loss in (B.7) is minimised for:

$$
\begin{aligned}
& \kappa_{1}=\frac{(1-\lambda r)^{2} \sigma_{\theta}^{2} \sigma_{\phi}^{2}}{\sigma_{\varepsilon}^{2}\left(\sigma_{\theta}^{2}+\sigma_{\phi}^{2}\right)+(1-\lambda r)^{2} \sigma_{\theta}^{2} \sigma_{\phi}^{2}} \\
& \kappa_{2}=\frac{\sigma_{\varepsilon}^{2} \sigma_{\theta}^{2}}{\sigma_{\varepsilon}^{2}\left(\sigma_{\theta}^{2}+\sigma_{\phi}^{2}\right)+(1-\lambda r)^{2} \sigma_{\theta}^{2} \sigma_{\phi}^{2}} .
\end{aligned}
$$

In the limit as $\sigma_{\theta}^{2}$ approaches infinity, (B.8a) and (B.8b) become:

$$
\begin{aligned}
& \kappa_{1}=\frac{(1-\lambda r)^{2} \sigma_{\phi}^{2}}{\sigma_{\varepsilon}^{2}+(1-\lambda r)^{2} \sigma_{\phi}^{2}} \\
& \kappa_{2}=\frac{\sigma_{\varepsilon}^{2}}{\sigma_{\varepsilon}^{2}+(1-\lambda r)^{2} \sigma_{\phi}^{2}} .
\end{aligned}
$$

Thus, socially efficient action is given by:

$$
\tilde{a}_{i}=\frac{(1-\lambda r)^{2} \sigma_{\phi}^{2} x_{i}+\sigma_{\varepsilon}^{2} z}{\sigma_{\varepsilon}^{2}+(1-\lambda r)^{2} \sigma_{\phi}^{2}} .
$$

We conjecture that socially efficient action can also be expressed as a linear function of agent $i$ 's expectation of the state variable $\theta$ and the average action $\bar{a}$ :

$$
\tilde{a}_{i}=\omega_{1} E_{i}[\theta]+\omega_{2} E_{i}[\bar{a}]
$$

where $E_{i}[\cdot]$ is the expectations conditional on signals $x_{i}$ and $z$. In this representation $\omega_{2}$ is effectively the collectively efficient degree of coordination that we are deriving. We assume that the expected value of $\theta$ is (see Appendix B.3):

$$
E_{i}[\theta]=\frac{\sigma_{\phi}^{2} x_{i}+\sigma_{\varepsilon}^{2} z}{\sigma_{\varepsilon}^{2}+\sigma_{\phi}^{2}},
$$

which is a weighted average of the two observed signals. Using (B.5), we write the expected value of $\bar{a}$ as:

$$
E_{i}[\bar{a}]=\kappa_{1} E_{i}[\theta]+\kappa_{2} z .
$$

Finally we substitute (B.12) and (B.13) into (B.11) and compare the coefficients on $x_{i}$ and $z$ in the resulting expression with those in (B.10). We then solve the system of equation for $\omega_{2}$ and obtain:

$$
C E D o C=\lambda r(2-\lambda r)
$$

which is equal to zero for $\lambda=0$ and $(2-r) r$ for $\lambda=1$. This is consistent with the characteristics of the M-S and J-L payoff functions, respectively. 
Of interest is the case where the equilibrium and the collectively efficient degrees of coordination are equal. Setting expressions (B.2) and (B.14) equal to each other yields a quadratic equation with two roots:

$$
\begin{aligned}
\lambda_{1} & =\frac{1}{2} \\
\lambda_{2} & =\frac{1}{r},
\end{aligned}
$$

where $r \in(0,1)$. The first root $\lambda_{1}$ lies in between the two extremes - zero and unity - and corresponds to the following value of equilibrium and collectively efficient degrees of coordination:

$$
\left.E D o C\right|_{\lambda=\lambda_{1}}=\left.C E D o C\right|_{\lambda=\lambda_{1}}=\frac{1}{4}(4-r) r
$$

With $\lambda=\lambda_{1}$, the payoff function in (2.8) becomes:

$$
\left.u_{i}\right|_{\lambda=\lambda_{1}}=-\frac{2-r}{8}\left[2 a_{i}-(2-r)(\theta+g)-r \bar{a}\right]^{2}-\frac{r}{2}\left(L_{i}-\bar{L}\right) .
$$

The second root $\lambda_{2}$ lies in the interval $[1,+\infty]$ and corresponds to equilibrium and collectively efficient degrees of coordination that are equal to unity. With $\lambda=\lambda_{2}$, the payoff function (2.8) becomes:

$$
\left.u_{i}\right|_{\lambda=\lambda_{2}}=-(2-r)\left(a_{i}-\bar{a}\right)^{2}+(1-r)\left(L_{i}-\bar{L}\right) .
$$

An equilibrium degree of coordination equal to unity implies that the agent only cares about coordination, which is consistent with the absence of the state variable $\theta$ and the policy instrument $g$ in the payoff function. Aggregating the payoff function in (B.19) results in a social welfare function that, similarly, does not contain $\theta$ nor $g$, which is consistent with a collectively efficient degree of coordination that is equal to unity.

\section{B.2 Derivation of the ex ante expected equilib- rium welfare}

We begin by substituting active agent $i$ 's optimal action from (2.25)

$$
a_{i}^{A}=\kappa_{0}+\kappa_{1} x_{i}+\kappa_{2} y
$$


average action from (2.27):

$$
\bar{a}=(1-Q)\left(\kappa_{0}+\kappa_{1} \theta+\kappa_{2} y\right),
$$

and the optimal policy expression from (2.31):

$$
g=\rho_{0}+\rho_{1} z+\rho_{2} y
$$

into the welfare equation from (2.28):

$$
W=-Q[(1-\lambda r)(\theta+g)+\lambda r \bar{a}]^{2}-\int_{Q}^{1}\left[a_{i}^{A}-(1-\lambda r)(\theta+g)-\lambda r \bar{a}\right]^{2} d i .
$$

We proceed by inserting the definitions of signals:

$$
\begin{aligned}
x_{i} & =\theta+\varepsilon_{i} \\
y & =\theta+\phi+\eta \\
z & =\theta+\phi,
\end{aligned}
$$

into the expanded welfare equation and take unconditional expectations of the resulting expression. The equilibrium expected welfare is given by:

$$
\begin{aligned}
E[W]= & -Q\left\{\left[\rho_{0}+\lambda r\left((1-Q) \kappa_{0}-\rho_{0}\right)\right]^{2}+\left[1+\rho_{1}+\rho_{2}-\lambda r\left(1-(1-Q) \kappa_{1}-\right.\right.\right. \\
& \left.\left.-(1-Q) \kappa_{2}\right)+\rho_{1}+\rho_{2}\right]^{2} \sigma_{\theta}^{2}+\left[\rho_{1}+\rho_{2}+\lambda r\left((1-Q) \kappa_{2}-\rho_{1}-\rho_{2}\right)\right]^{2} \sigma_{\phi}^{2}+ \\
& \left.+\left[\rho_{2}+\lambda r\left((1-Q) \kappa_{2}-\rho_{2}\right)\right]^{2} \sigma_{\eta}^{2}\right\}-(1-Q)\left\{\left[\kappa_{0}-(1-Q) \lambda r \kappa_{0}-\right.\right. \\
& \left.-(1-\lambda r) \rho_{0}\right]^{2}+\left[\kappa_{1}+\kappa_{2}-\lambda r(1-Q) \kappa_{1}-\lambda r(1-Q) \kappa_{2}-(1-\lambda r) \cdot\right. \\
& \left.\cdot\left(1+\rho_{1}+\rho_{2}\right)\right]^{2} \sigma_{\theta}^{2}+\kappa_{1}^{2} \sigma_{\varepsilon}^{2}+\left[\kappa_{2}-\lambda r(1-Q) \kappa_{2}-(1-\lambda r)\left(\rho_{1}+\rho_{2}\right)\right]^{2} \sigma_{\phi}^{2}+ \\
& \left.+\left[\kappa_{2}-\lambda r(1-Q) \kappa_{2}-(1-\lambda r) \rho_{2}\right]^{2} \sigma_{\eta}^{2}\right\} .
\end{aligned}
$$

Since $\sigma_{\theta}^{2}$ is infinite, in order to ensure that equilibrium expected welfare always has a finite value the combined coefficient on $\sigma_{\theta}^{2}$ has to amount to zero. This requirement implies the following constraints on equilibrium parameters:

$$
\begin{aligned}
& \kappa_{1}+\kappa_{2}=0 \\
& \rho_{1}+\rho_{2}=-1 .
\end{aligned}
$$


Unless the conditions in (B.21)-(B.22) are satisfied, the society's expected loss is undefined.

\section{B.3 Derivation of the expected values of $\theta$ and $z$}

Active agents form an expectation of the state variable $\theta$ conditional on the observed signals $x_{i}$ and $y$. Thus, we begin by assuming that agent $i$ 's expectation of $\theta$ can be expressed as a linear combination of the two signals:

$$
E_{i}[\theta]=a x_{i}+b y
$$

where $a$ is used here for convenience of notation only, is not part of the model discussed in the main sections and must not be confused with agent $i$ 's action denoted by $a_{i}^{k}$, where $k=A, P$. Next we need to find $a$ and $b$ so as to minimise the mean squared error (MSE) of $E_{i}[\theta]$ as an estimator of $\theta$ :

$$
\max M S E\left[E_{i}[\theta]\right] \quad \text { w.r.t. } a \text { and } b,
$$

where the mean squared error is defined as:

$$
M S E\left[E_{i}[\theta]\right]=E_{i}\left[\theta-E_{i}[\theta]\right]^{2}
$$

We begin by substituting (B.23) and the definitions of the two signals from (2.22) and (2.30):

$$
\begin{aligned}
x_{i} & =\theta+\varepsilon_{i} \\
y & =\theta+\phi+\eta,
\end{aligned}
$$

into (B.25):

$$
M S E=E_{i}\left[\theta-a x_{i}-b y\right]^{2}=E_{i}\left[(1-a-b) \theta-a \varepsilon_{i}-b(\phi+\eta)\right]^{2} .
$$

We expand the expression in (B.26), take expectations and exclude summands that are equal to zero due to independence of noises:

$M S E=(1-a-b)^{2} E_{i}\left[\theta^{2}\right]+a^{2} E_{i}\left[\varepsilon_{1}^{2}\right]+b^{2} E_{i}\left[\phi^{2}+\eta^{2}\right]=(1-a-b)^{2} \sigma_{\theta}^{2}+a^{2} \sigma_{\varepsilon}^{2}+b\left(\sigma_{\phi}^{2}+\sigma_{\eta}^{2}\right)$.

Considering that $\sigma_{\theta}^{2}$ is infinite, we need to impose a restriction on $a$ and $b$ in order to ensure that the MSE takes on a finite value. Thus, we make an additional assumption that $a+b=1$. Having found the expression for MSE in terms of noise 
variances, we now derive the first-order conditions for the optimisation problem in (B.24):

$$
\begin{aligned}
& \frac{\partial M S E}{\partial a}=-2(1-a-b) \sigma_{\theta}^{2}+a \sigma_{\varepsilon}^{2}=0 \\
& \frac{\partial M S E}{\partial b}=-2(1-a-b) \sigma_{\theta}^{2}+b\left(\sigma_{\phi}^{2}+\sigma_{\eta}^{2}\right)=0 .
\end{aligned}
$$

Combining the two first-order conditions yields:

$$
a \sigma_{\varepsilon}^{2}=b\left(\sigma_{\phi}^{2}+\sigma_{\eta}^{2}\right)
$$

Using the constraint on $a$ and $b$ that we introduced earlier, we derive the following expressions for the the two coefficients:

$$
\begin{aligned}
a & =\frac{\sigma_{\phi}^{2}+\sigma_{\eta}^{2}}{\sigma_{\varepsilon}^{2}+\sigma_{\phi}^{2}+\sigma_{\eta}^{2}} \\
b & =\frac{\sigma_{\varepsilon}^{2}}{\sigma_{\varepsilon}^{2}+\sigma_{\phi}^{2}+\sigma_{\eta}^{2}} .
\end{aligned}
$$

By substituting (B.31a) and (B.31b) into equation (B.23) we arrive at the desired expression for agent $i$ 's conditional expectation of the state variable $\theta$ from (2.32):

$$
E_{i}[\theta]=\frac{\left(\sigma_{\phi}^{2}+\sigma_{\eta}^{2}\right) x_{i}+\sigma_{\varepsilon}^{2} y}{\sigma_{\varepsilon}^{2}+\sigma_{\phi}^{2}+\sigma_{\eta}^{2}},
$$

where $i \in(Q, 1]$ and the right-hand side is a weighted average of the two observed signals.

Similarly, in order to find the expected value of the CB's private signal $z$, conditional on the observed signals, we need to solve the following problem:

$$
\max \quad M S E\left[E_{i}[z]\right] \quad \text { w.r.t. } \quad c \text { and } d,
$$

where $i \in(Q, 1]$. For this purpose we need the definition of $z$ from (2.29):

$$
z=\theta+\phi
$$

and:

$$
\begin{aligned}
E_{i}[z] & =c x_{i}++d y \\
M S E\left[E_{i}[z]\right] & =E_{i}\left[z-E_{i}[z]\right]^{2} .
\end{aligned}
$$


Following the same steps as taken above in the derivation of $E_{i}[\theta]$ yields the expression from (2.33):

$$
E_{i}[z]=\frac{\sigma_{\eta}^{2} x_{i}+\left(\sigma_{\varepsilon}^{2}+\sigma_{\phi}^{2}\right) y}{\sigma_{\varepsilon}^{2}+\sigma_{\phi}^{2}+\sigma_{\eta}^{2}},
$$

where $i \in(Q, 1]$.

\section{B.4 Commitment to a rule: optimal transparency for $0<\lambda<1$}

Here we present the conditions for optimality of each transparency regime in the setting with information stickiness $(0<Q<1)$ and commitment to a policy rule for intermediate cases of the hybrid payoff function $0<\lambda<1$.

As mentioned above, partial transparency is optimal, when the optimum (one of the two solutions to the first-order condition with respect to $\sigma_{\eta}^{2}$ ) is positive, which is true when one of the following six sets of conditions are satisfied:

$$
\begin{aligned}
& \left\{\begin{array}{l}
0<r<\frac{1}{2} \\
0 \leq \lambda<\frac{1}{2} \\
0<Q<Q_{B 1} \\
0<\sigma_{\phi}^{2}<\sigma_{\phi B 2}^{2},
\end{array}\right. \\
& \left\{\begin{array}{l}
0<r<\frac{1}{2} \\
\frac{1}{2}<\lambda \leq 1 \\
0<Q<Q_{B 1} \\
0<\sigma_{\phi}^{2}<\sigma_{\phi B 2}^{2}
\end{array}\right. \\
& \left\{\begin{array}{l}
\frac{1}{2} \leq r<1 \\
0 \leq \lambda<\frac{1}{2} \\
0<Q<Q_{B 1} \\
0<\sigma_{\phi}^{2}<\sigma_{\phi B 2}^{2},
\end{array}\right. \\
& \left\{\begin{array}{l}
\frac{1}{2} \leq r<1 \\
\frac{1}{2}<\lambda<\frac{1+r}{3 r} \\
0<Q<Q_{B 1} \\
0<\sigma_{\phi}^{2}<\sigma_{\phi B 2}^{2},
\end{array}\right.
\end{aligned}
$$


where:

$$
\begin{aligned}
Q_{B 1} & \equiv \frac{(1-2 \lambda r)^{2}[1-(1-\lambda) r] r^{2}}{1+(1-3 \lambda) r+(1-\lambda)^{2} r^{2}-(1-\lambda)(1-2 \lambda)^{2} r^{3}} \\
Q_{B 2} & \equiv\left(Q_{1}\right)=\frac{(1-\lambda r)^{2}[1-(1-\lambda) r]}{\left[(2-\lambda) \lambda r-(1-\lambda) \lambda^{2} r^{2}-2(1-\lambda)\right] r} \\
\sigma_{\phi B 1}^{2} & \equiv \frac{(3 \lambda r-1-r) \sigma_{\varepsilon}^{2}}{\left.1-[1-2 Q(1-\lambda)+\lambda] r+(2-\lambda) \lambda(1-Q) r^{2}-(1-\lambda) \lambda^{2}(1-Q) r^{3}\right]} \\
\sigma_{\phi B 2}^{2} & \equiv(1-Q)(1-\lambda r)[1-(1-\lambda) r] r . \\
& \cdot \sqrt{\frac{(1-2 \lambda)^{2} \sigma_{\phi B 1}^{4}}{(1+r-3 \lambda r)^{2}\{1-(1-\lambda)(1-Q)[2-(1-\lambda) r] r\} Q}}+\sigma_{\phi B 1}^{2},
\end{aligned}
$$

where the argument under the square root sign in $\sigma_{\phi B 2}^{2}$ is not defined for $Q=Q_{B 2}$. Zero transparency is optimal when:

$$
\left\{\begin{array}{l}
\frac{1}{2} \leq r<1 \\
\frac{1+r}{3 r}<\lambda \leq 1 \\
0<Q_{B 2} \leq Q,
\end{array}\right.
$$

which is equivalent to conditions (2.86a)-(2.86c), that ensure optimality of zero transparency under discretionary monetary policy. Finally, full transparency is optimal for all remaining combinations of parameters, which are described by the following twelve sets of conditions:

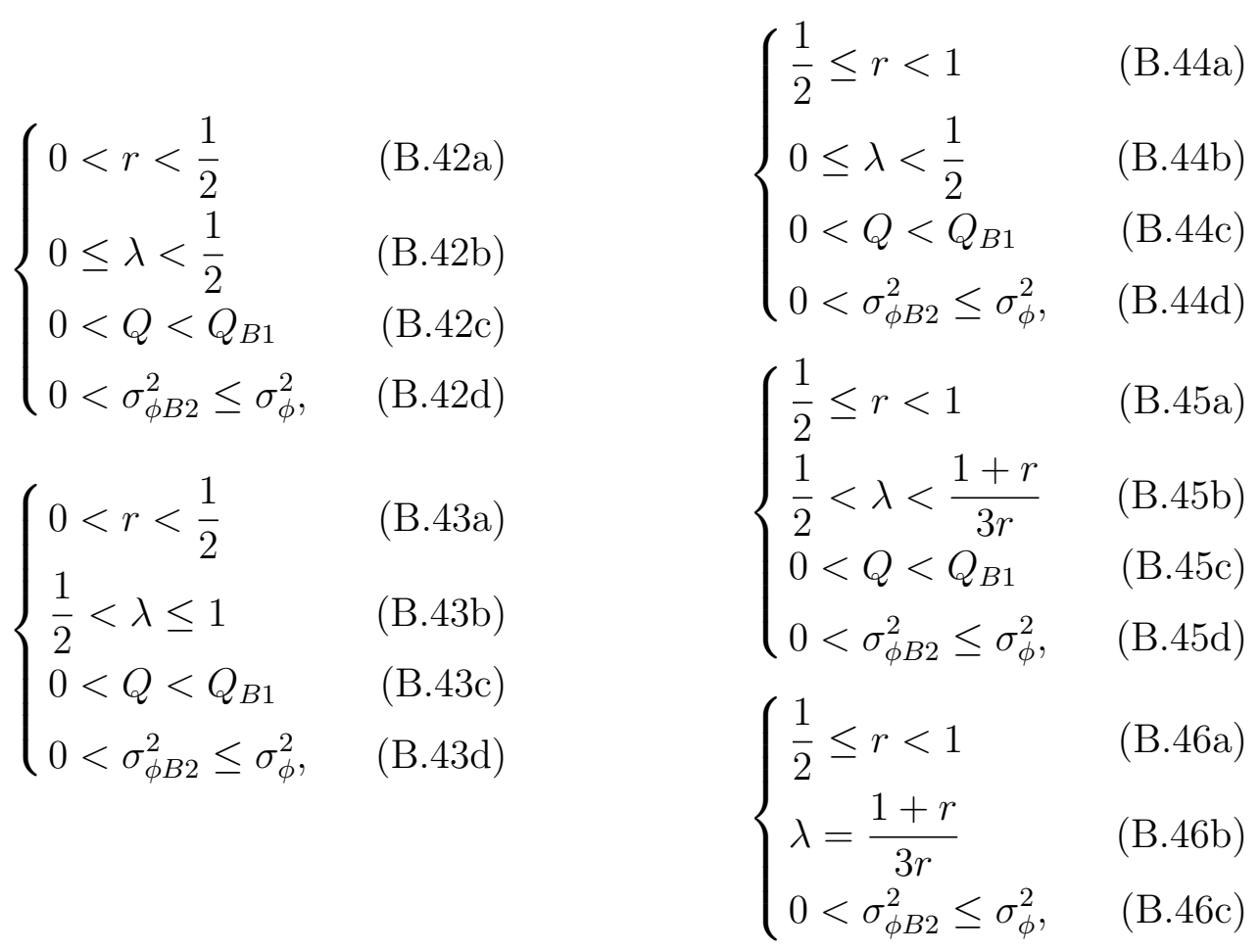




$$
\begin{aligned}
& \left\{\begin{array}{l}
\frac{1}{2} \leq r<1 \\
\frac{1+r}{3 r}<\lambda \leq 1 \\
0<Q<Q_{B 2} \\
0<\sigma_{\phi B 2}^{2} \leq \sigma_{\phi}^{2},
\end{array}\right. \\
& \left\{\begin{array}{l}
0<r<\frac{1}{2} \\
\frac{1}{2}<\lambda \leq 1 \\
0<Q_{B 1} \leq Q<1,
\end{array}\right. \\
& \left\{\begin{array}{l}
\frac{1}{2} \leq r<1 \\
0 \leq \lambda<\frac{1}{2} \\
0<Q_{B 1} \leq Q<1,
\end{array}\right. \\
& \left\{\begin{array}{l}
0<r<\frac{1}{2} \\
0 \leq \lambda<\frac{1}{2} \\
0<Q_{B 1} \leq Q<1,
\end{array}\right. \\
& \left\{\begin{array}{l}
0<r<\frac{1}{2} \\
\lambda=\frac{1}{2},
\end{array}\right. \\
& \left\{\begin{array}{l}
\frac{1}{2} \leq r<1 \\
\lambda=\frac{1}{2},
\end{array}\right. \\
& \left\{\begin{array}{l}
\frac{1}{2} \leq r<1 \\
\frac{1}{2}<\lambda<\frac{1+r}{3 r} \\
0<Q_{B 1} \leq Q<1,
\end{array}\right.
\end{aligned}
$$

\section{B.5 Commitment to a rule: examples of optimal transparency (J-L case)}

In the case of the J-L function and commitment to a monetary policy rule on part of the $\mathrm{CB}$, optimal disclosure policy is defined by a set of conditions listed in general form in Table 2.1. The conditions are formulated in terms of critical values for the coordination parameter $r$.

Table B.1 presents a numerical example for the case, where policymaker's information is more accurate than the active agents' private information $\left(\sigma_{\phi}^{2}<\sigma_{\varepsilon}^{2}\right)$.

Table B.1: Conditions for optimal disclosure policies under commitment in the J-L case. Numerical example: $\sigma_{\varepsilon}^{2}=4, \sigma_{\phi}^{2}=1, Q=\frac{1}{4}$

\begin{tabular}{|c|c|c|c|}
\hline & Full: $\sigma_{\eta}^{2}=0$ & Partial: $\sigma_{\eta}^{2}=\left(\sigma_{\eta}^{*}\right)^{2}$ & Zero: $\sigma_{\eta}^{2} \rightarrow \infty$ \\
Condition & $0<r \leq \frac{1}{3}$ & $\frac{1}{3}<r<\frac{2}{3}$ & $\frac{2}{3} \leq r<1$ \\
\hline
\end{tabular}

Figure B.1 shows the expected welfare outcome as a function of the coordination parameter under three disclosure regimes: (i) full transparency, (ii) partial trans- 
parency and (iii) zero transparency, or full opacity ${ }^{1}$. The first panel shows that the shapes of the three expected welfare curves are different depending on the disclosure regime. The vertical lines correspond to the critical values $r_{1}=\frac{1}{2}$ and $r_{2}=\frac{4}{5}$, from left to right. Partial transparency dominates full transparency for all parameter combinations except for the critical value $r_{1}=\frac{1}{2}$, in which case the welfare outcomes are the same. However, it should be noted that the welfare outcome under partial transparency is not defined outside the interval $r \in\left[\frac{1}{2}, \frac{4}{5}\right)$ due to the optimal variance of the additional noise $\sigma_{\eta}^{2}$ being negative. Thus, the full curve is plotted merely for clarity of the graph. The relationship between the outcomes under full and zero transparency is ambiguous, with zero transparency being superior for $r \in\left(\frac{2}{3}, 1\right)$. The second panel superposes the expected welfare curve that corresponds to optimal disclosure policy on top of the first panel, showing all three regimes are

\footnotetext{
${ }^{1}$ See equations (2.59)-(2.61), respectively.
}

Figure B.1: J-L case and commitment. Expected welfare under transparency regimes: $\sigma_{\varepsilon}^{2}=1, \sigma_{\phi}^{2}=4, Q=\frac{1}{16}$
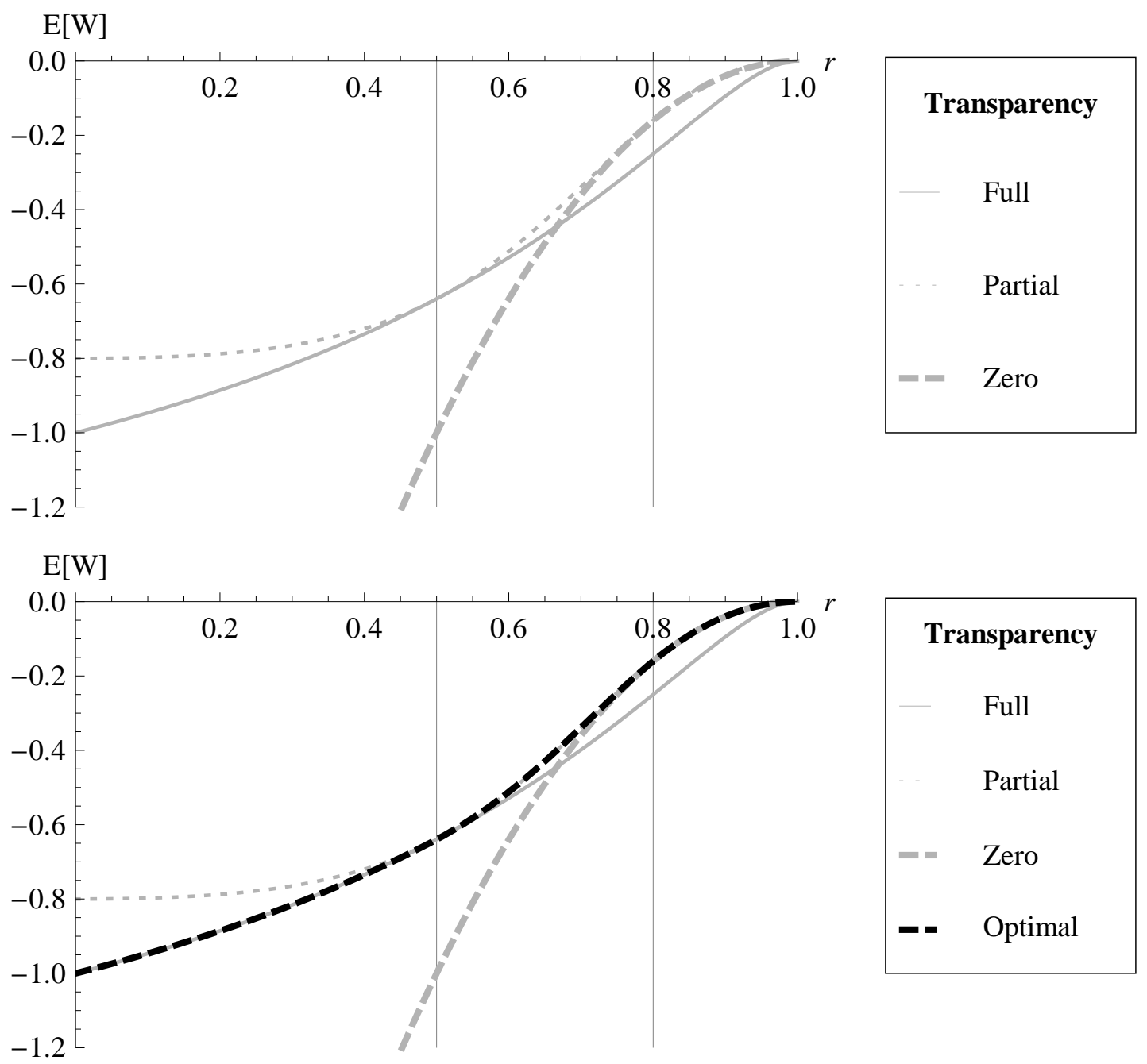
optimal depending on the value of $r$ relative to the values of other parameters.

\section{B.6 Discretion: shape of the expected equilib- rium welfare function (hybrid case)}

Expected equilibrium welfare in $(2.80)$ is continuous in $\sigma_{\eta}^{2}$ and reaches a minimum value at the stationary point $\sigma_{\eta 1}^{2}$, given in $(2.81)$ and restricted to $0 \leq \sigma_{\eta 1}^{2}$. The solution to the first-order condition $\sigma_{\eta 1}^{2}$ is positive, when:

$$
\left\{\begin{array}{l}
\frac{2}{3}<\lambda<1 \\
\frac{1}{3 \lambda-1}<r<1 \\
0<Q<Q_{C 1} \\
0<\sigma_{\phi C 1}^{2}<\sigma_{\phi}^{2} .
\end{array}\right.
$$

where:

$$
\begin{aligned}
Q_{C 1} & \equiv \frac{[1-(1-\lambda) r]^{2}(1-\lambda r)}{\left[4 \lambda-(1-\lambda)^{2} \lambda r^{2}+\left(1-\lambda^{2}\right) r-3\right] r} \\
\sigma_{\phi C 1}^{2} & \equiv \frac{(3 \lambda r-1-r) \sigma_{\varepsilon}^{2}}{1-[2-\lambda-(3-4 \lambda) Q] r+\left(1-\lambda^{2}\right)(1-Q) r^{2}-(1-\lambda)^{2}(1-Q) \lambda r^{3}} .
\end{aligned}
$$

Under conditions (B.54a)-(B.54b), $Q_{2}<Q_{C 1}, \sigma_{\phi C 1}^{2}<\sigma_{\phi 2}^{2}$ for $0<Q<Q_{2}$ and $\sigma_{\phi 2}^{2}<\sigma_{\phi C 1}^{2}$ for $Q_{2}<Q<Q_{C 1}$, where:

$$
\begin{aligned}
Q_{2} & \equiv \frac{[1-(1-\lambda) r](1-\lambda r)^{2}}{2+[2-(1+r(1-\lambda)) \lambda] r\} \lambda r-2 r} \\
\sigma_{\phi 2}^{2} & \equiv \frac{(3 \lambda r-1-r) \sigma_{\varepsilon}^{2}}{1-[1-2 Q(1-\lambda)+\lambda] r+(2-\lambda) \lambda(1-Q) r^{2}-(1-\lambda) \lambda^{2}(1-Q) r^{3}}
\end{aligned}
$$

When conditions (B.54a)-(B.54d) are not met, the stationary point $\sigma_{\eta 1}^{2}$ is not defined due to it being a noise variance and non-negative by definition. When $\sigma_{\eta 1}^{2}$ is negative, expected equilibrium welfare is monotonic in the variance of the additional noise over the range $0 \leq \sigma_{\eta}^{2}$.

For a given combination of values of $\lambda, Q, r, \sigma_{\varepsilon}^{2}$ and $\sigma_{\phi}^{2}$, the expected equilibrium welfare function takes a unique shape in respect to $0 \leq \sigma_{\eta}^{2}$. All possible shapes can be broadly classified into following four groups:

- monotonically decreasing in $\sigma_{\eta}^{2}$;

- achieving a minimum at $\sigma_{\eta 1}^{2}$ with intercept at $\sigma_{\eta}^{2}=0$ above the horizontal 
asymptote;

- achieving a minimum at $\sigma_{\eta 1}^{2}$ with intercept at $\sigma_{\eta}^{2}=0$ below the horizontal asymptote; and

- monotonically increasing in $\sigma_{\eta}^{2}$.

In the former two cases maximum welfare is achieved at full transparency $\left(\sigma_{\eta}^{2}=0\right)$, whereas in the latter two cases full opacity is optimal $\left(\sigma_{\eta}^{2} \rightarrow \infty\right)$. The optimal choice of disclosure regimes is described by conditions (2.85a)-(2.90d). Figure B.2 presents graphs of numerical examples corresponding to the four cases.

Expected equilibrium welfare is monotonically decreasing in $\sigma_{\eta}^{2}$, when:

$$
0 \leq \lambda \leq \frac{2}{3}
$$

or:

$$
\left\{\begin{array}{l}
\frac{2}{3}<\lambda \leq 1 \\
0<r \leq \frac{1}{3 \lambda-1}
\end{array}\right.
$$

The minimum at $\sigma_{\eta 1}^{2}$ is defined and the intercept at $\sigma_{\eta}^{2}=0$ lies above the horizontal asymptote for:

$$
\left\{\begin{array}{l}
\frac{2}{3}<\lambda \leq 1 \\
\frac{1}{3 \lambda-1}<r<1 \\
0<Q<Q_{2} \\
0<\sigma_{\phi 2}^{2}<\sigma_{\phi}^{2}
\end{array}\right.
$$

The minimum at $\sigma_{\eta 1}^{2}$ is defined and the intercept at $\sigma_{\eta}^{2}=0$ lies below the horizontal asymptote if one of the two following sets of conditions is met:

$$
\left\{\begin{array} { l l } 
{ \frac { 2 } { 3 } < \lambda \leq 1 } & { \text { (B.58a) } } \\
{ \frac { 1 } { 3 \lambda - 1 } < r < 1 } & { ( \mathrm { B } . 5 8 \mathrm { b } ) } \\
{ 0 < Q < Q _ { 2 } } & { ( \mathrm { B } . 5 8 \mathrm { c } ) } \\
{ 0 < \sigma _ { \phi C 1 } ^ { 2 } < \sigma _ { \phi } ^ { 2 } < \sigma _ { \phi 2 } ^ { 2 } . } & { ( \mathrm { B } . 5 8 \mathrm { d } ) }
\end{array} \quad \left\{\begin{array}{l}
\frac{2}{3}<\lambda \leq 1 \\
\frac{1}{3 \lambda-1}<r<1 \\
0<Q_{2}<Q<Q_{C 1} \\
0<\sigma_{\phi C 1}^{2}<\sigma_{\phi}^{2} .
\end{array}\right.\right.
$$




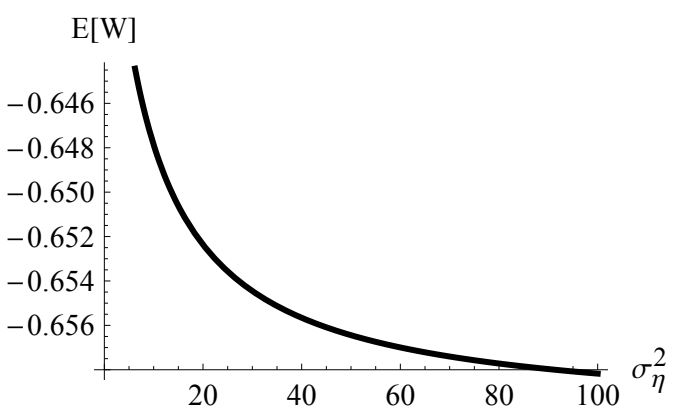

a) $\lambda=\frac{3}{4}, r=\frac{1}{4}, Q=\frac{2}{3}, \sigma_{\varepsilon}^{2}=4$ and $\sigma_{\phi}^{2}=1$

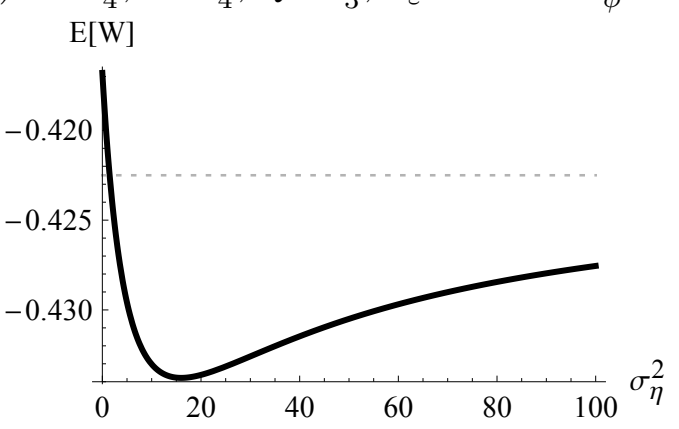

b) $\lambda=\frac{3}{4}, r=\frac{9}{10}, Q=\frac{2}{10}, \sigma_{\varepsilon}^{2}=1$ and $\sigma_{\phi}^{2}=4$

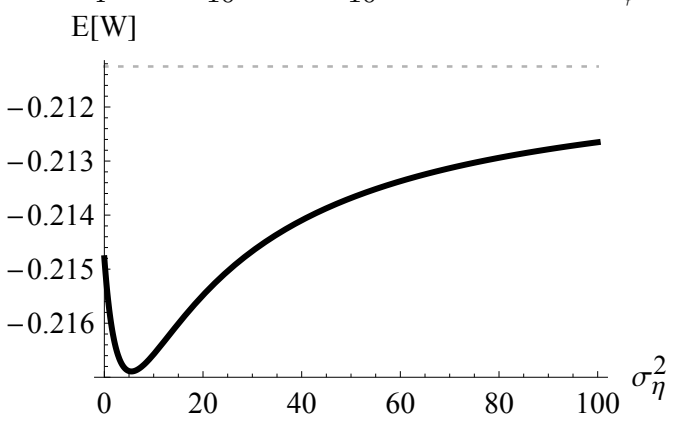

c) $\lambda=\frac{3}{4}, r=\frac{9}{10}, Q=\frac{2}{10}, \sigma_{\varepsilon}^{2}=1$ and $\sigma_{\phi}^{2}=2$

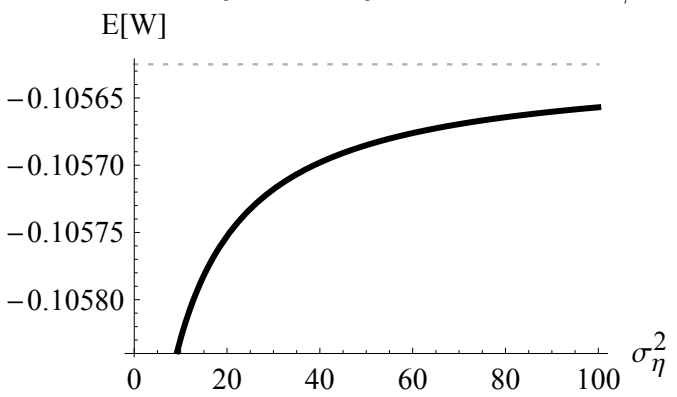

d) $\lambda=\frac{3}{4}, r=\frac{9}{10}, Q=\frac{3}{4}, \sigma_{\varepsilon}^{2}=4$ and $\sigma_{\phi}^{2}=1$

Figure B.2: Hybrid case and discretion. Shapes of the expected welfare function

Expected equilibrium welfare is monotonically increasing in $\sigma_{\eta}^{2}$ if one of the following three sets of conditions is satisfied: 


$$
\begin{aligned}
& \left\{\begin{array}{l}
\frac{2}{3}<\lambda \leq 1 \\
\frac{1}{3 \lambda-1}<r<1 \\
0<Q<Q_{2} \\
0<\sigma_{\phi}^{2}<\sigma_{\phi C 1}^{2} .
\end{array}\right. \\
& \left\{\begin{array}{l}
\frac{2}{3}<\lambda \leq 1 \\
\frac{1}{3 \lambda-1}<r<1 \\
0<Q_{C 1}<Q .
\end{array}\right. \\
& \left\{\begin{array}{l}
\frac{2}{3}<\lambda \leq 1 \\
\frac{1}{3 \lambda-1}<r<1 \\
0<Q_{2}<Q<Q_{C 1} \\
0<\sigma_{\phi}^{2}<\sigma_{\phi C 1}^{2} .
\end{array}\right.
\end{aligned}
$$

\section{B.7 Discretion: examples of optimal transparency (J-L case)}

In the case of the J-L payoff function and discretionary monetary policy, in equilibrium optimal disclosure policy is defined by a condition presented in general form in Table 2.2. For convenience, the condition is formulated in terms of a critical value for the coordination parameter $r$.

Table B.2 presents a numerical example for the case, where policymaker's information is more accurate than the active agents' private information $\left(\sigma_{\phi}^{2}<\sigma_{\varepsilon}^{2}\right)$.

Table B.2: Conditions for optimal disclosure policies under discretion in the J-L case. Numerical example: $\sigma_{\varepsilon}^{2}=4, \sigma_{\phi}^{2}=1, Q=\frac{1}{20}$

\begin{tabular}{|c|c|c|}
\hline & Full: $\sigma_{\eta}^{2}=0$ & Zero: $\sigma_{\eta}^{2} \rightarrow \infty$ \\
Condition & $0<r \leq \frac{10}{19} \approx 0.53$ & $\frac{10}{19} \leq r<1$ \\
\hline
\end{tabular}

Figure B.3 shows the expected welfare outcome as a function of the coordination 
Figure B.3: J-L case and discretion. Expected welfare under transparency regimes: $\sigma_{\varepsilon}^{2}=1, \sigma_{\phi}^{2}=4, Q=\frac{1}{16}$
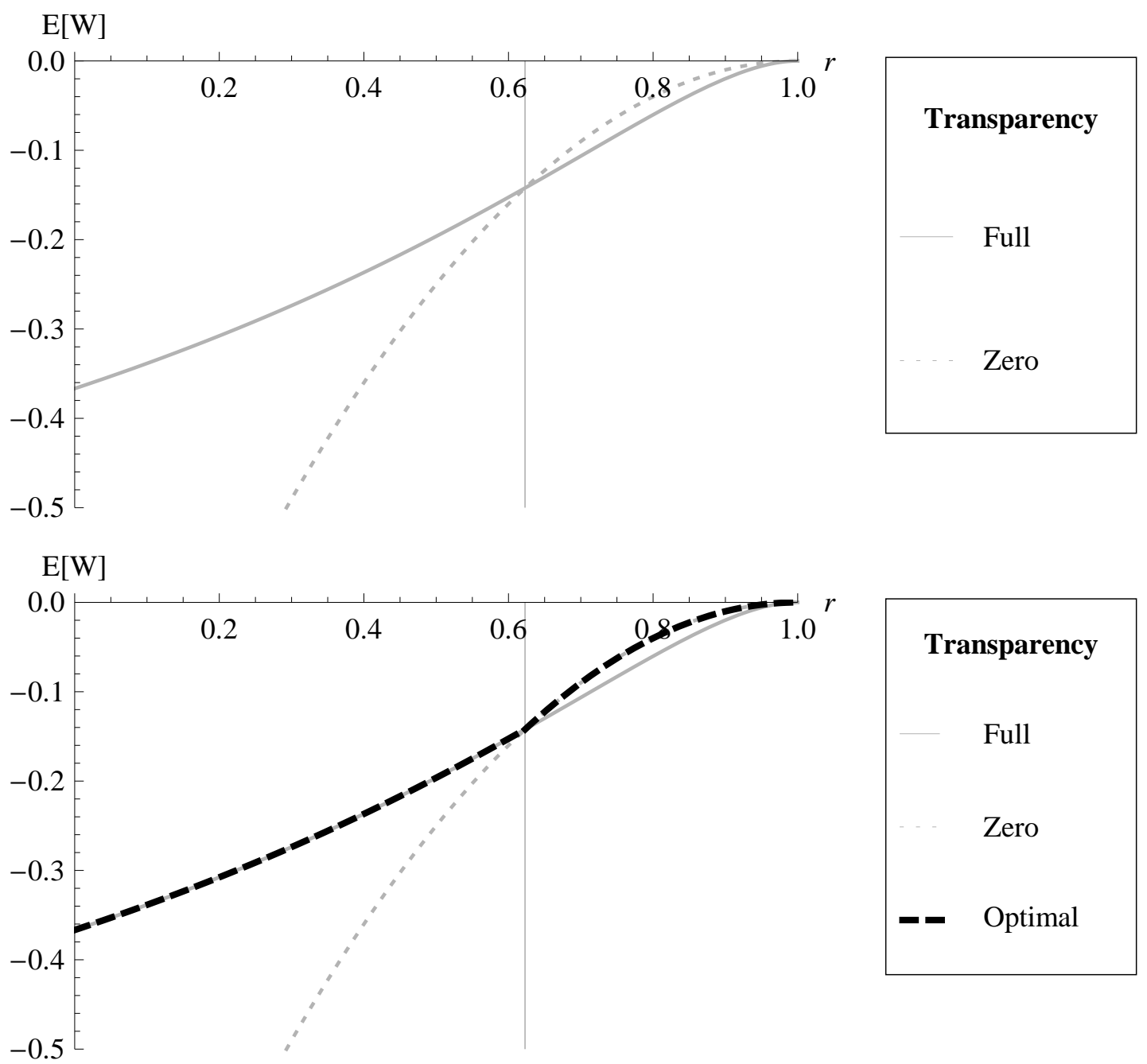

parameter under two disclosure regimes: (i) full transparency and (ii) zero transparency, or full opacity ${ }^{2}$. The parameter values used in the construction of the graphs correspond to the case where active agents have access to private information that is more accurate than the policymaker's own information $\left(\sigma_{\phi}^{2}<\sigma_{\varepsilon}^{2}\right)$. The first panel shows that the shapes of the two expected welfare curves are different depending on the disclosure regime. The vertical line corresponds to the critical value $r_{3} \approx 0.62$. The relationship between the outcomes under full and zero transparency is ambiguous, with zero transparency being superior in the region to the right of the vertical line. The second panel superposes the expected welfare curve that corresponds to optimal disclosure policy on top of the first panel. Thus, Figure B.3 demonstrates that the two regimes are optimal depending on the value of $r$ relative to the values of other parameters.

\footnotetext{
${ }^{2}$ See equations (2.95) and (2.96), respectively
} 


\section{B.8 Example of policy performance under com- mitment and discretion (M-S case)}

Here we give a numerical example of a market, where the CB's private information is more accurate than that of individual market participants $\left(\sigma_{\phi}^{2}<\sigma_{\varepsilon}^{2}\right)$. Figure B.4 presents an example of welfare outcomes under commitment and discretionary monetary policy to a rule for the M-S case $^{3}$. Given specific values of $\sigma_{\varepsilon}^{2}, \sigma_{\phi}^{2}$ and

Figure B.4: Policy performance in the M-S case: $\sigma_{\varepsilon}^{2}=4, \sigma_{\phi}^{2}=1, r=\frac{7}{10}$

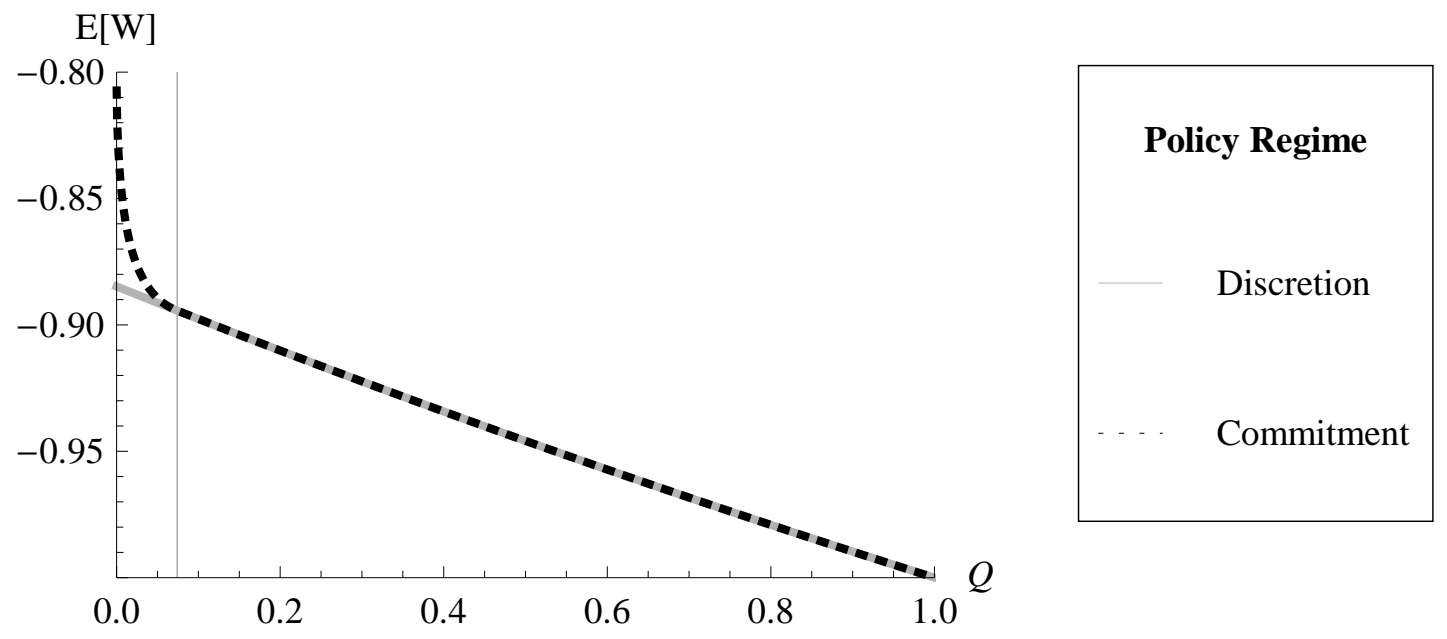

$r$ as well as discretionary policy, full transparency is optimal for $0<Q<1$ and the welfare outcome is represented by a straight downward-sloping line. However, under commitment partial disclosure is optimal for $0<Q<Q_{D 1}$, where $Q_{D 1}$ is the highest value of $Q$ that satisfies (2.53a)-(2.53b) ${ }^{4}$; and full disclosure is optimal for $Q_{D 1} \leq Q<1$. Thus, the curve representing commitment to a policy rule is a combination of a convex curve over the partial disclosure range and a straight line over the full disclosure range. The respective expected welfare outcomes in general form are given by equations (2.92) and (2.54)-(2.55). The vertical grid line on the graph corresponds to the critical value $Q_{D 1} \approx 0.074$. Figure B.4 demonstrates that commitment outperforms discretion over the interval $0<Q<Q_{D 1}$ and that the choice of the policy regime is arbitrary for $Q_{D 1} \leq Q<1$.

\footnotetext{
${ }^{3}$ It should be noted that, in contrast to the graphs above, here we plot the expected welfare outcome with the proportion of passive agents $Q$ instead of $r$ along the horizontal axis.

${ }^{4}$ The precise expression for $Q_{D 1}$ is omitted here due to its complexity in general form.
} 


\section{B.9 Example of policy performance under com- mitment and discretion ( J-L case)}

In this example we use a market, where market participants are better informed than the $\mathrm{CB}\left(\sigma_{\varepsilon}^{2}<\sigma_{\phi}^{2}\right)$. We demonstrate how the choice of a policy regime outlined in Table 2.4 affects the expected social welfare outcome. To do that we combine the graphs previously used in Appendix B.7 and Appendix B.5. The three vertical grid lines in Figure B.5 from left to right correspond to $r_{1} \approx 0.26, r_{3} \approx 0.69$ and $r_{2} \approx 0.93$, respectively. The welfare under commitment is a combination of three curves corresponding to three disclosure regimes: full transparency for $0<r \leq r_{1}$, partial transparency for $r_{1}<r<r_{2}$ and zero transparency for $r_{2} \leq r<1$. The curve representing the outcome under discretion is a combination of two curves corresponding to full transparency for $0<r \leq r_{3}$ and zero transparency for $r_{3} \leq$ $r<1$. It is apparent from the graph that commitment outperforms discretion over the range $r_{1}<r<r_{2}$.

Figure B.5: Policy performance in the J-L case: $\sigma_{\varepsilon}^{2}=1, \sigma_{\phi}^{2}=4, Q=\frac{1}{50}$

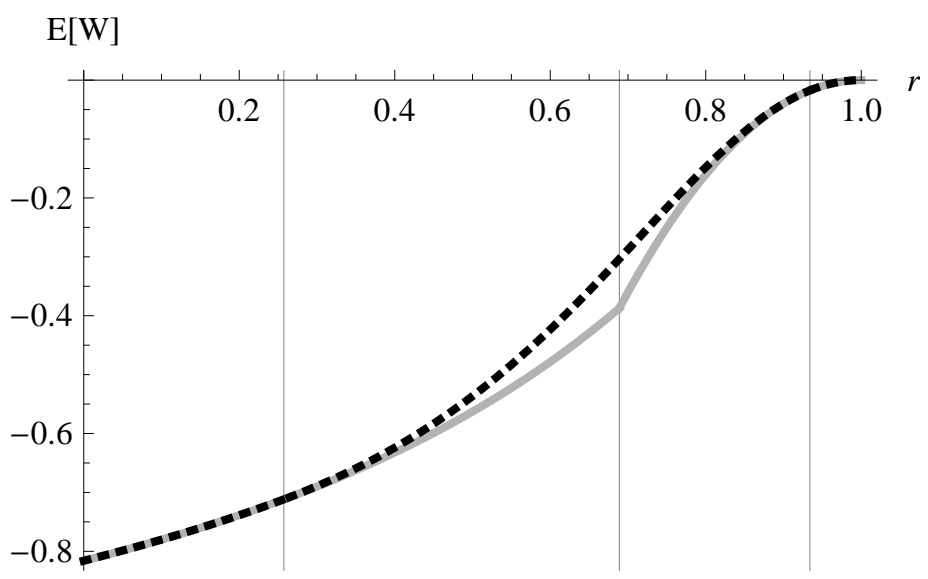

Policy Regime

Discretion

Commitment 


\section{Appendix C}

\section{C.1 Model: re-formulated payoff functions}

The original Morris and Shin (2002) payoff function is given by:

$$
u_{i}^{\mathrm{MS}}=-\left(1-r^{\mathrm{MS}}\right)\left(a_{i}-\theta\right)^{2}-r^{\mathrm{MS}}\left(L_{i}-\bar{L}\right)
$$

where $a_{i}$ is agent $i$ 's action, $\theta$ is the state variable, $0<r^{\mathrm{MS}}<1$ is the coordination coefficient, $L_{i}=\int_{0}^{1}\left(a_{j}-a_{i}\right)^{2} d j$, and $\bar{L}=\int_{0}^{1} L_{i} d i$. Note that $r^{\mathrm{MS}}$ is a proper fraction below unity, thus, the payoff is a weighted average of two components. The first component is a quadratic loss in the distance between agent $i$ 's action and the shock. The second component is the 'beauty contest' term, which is a loss from imperfect coordination between agent $i$ 's action and other agents' actions. The weight on the latter $r^{\mathrm{MS}}$ is the coordination parameter that measures the strength of the coordination motive, or, in other words, the relative importance of coordination, as perceived by individual agents.

Aggregating the payoff in (C.1) across the entire population yields the expression for social welfare:

$$
W^{\mathrm{MS}}=-\left(1-r^{\mathrm{MS}}\right) \int_{0}^{1}\left(a_{i}-\theta\right)^{2} d i .
$$

By dividing the above expression through by $\left(1-r^{\mathrm{MS}}\right)$, we are able to obtain a normalised expression:

$$
W^{\mathrm{MS}}=-\int_{0}^{1}\left(a_{i}-\theta\right)^{2} d i
$$

that is independent of the coordination parameter $r^{\mathrm{MS}}$. This is crucial, as the original M-S payoff function was constructed in a way so as to give perceived benefit to coordination at the individual, but not the aggregate level. Hence, the absence of $r^{\mathrm{MS}}$ in the social welfare expression is a central characteristic of the Morris-Shin model. 
Introducing a cost component into the payoff function (C.1), leads to a technical pitfall, when aggregating across the population level. In particular, a payoff, given by:

$$
u_{i}^{\mathrm{MS}}=-\left(1-r^{\mathrm{MS}}\right)\left(a_{i}-\theta\right)^{2}-r^{\mathrm{MS}}\left(L_{i}-\bar{L}\right)-C_{i},
$$

where $C_{i}$ is the individual cost component, when aggregated, becomes:

$$
W^{\mathrm{MS}}=-\left(1-r^{\mathrm{MS}}\right) \int_{0}^{1}\left(a_{i}-\theta\right)^{2} d i-\int_{0}^{1} C_{i} d i
$$

The above expression cannot be normalised so as to get rid of the coordination parameter, meaning that coordination is no longer neutral at the aggregate level. This contradicts the core characteristic of the original Morris-Shin model, and, thereby, creates unwanted consequences for the cost of information relative to the cost of imperfect alignment of agents' actions with the fundamental.

Our approach to solving the above issue is to re-formulate the payoff function in the way presented in (3.1):

$$
u_{i}^{\mathrm{MS}}=-\left(a_{i}-\theta\right)^{2}-r\left(L_{i}-\bar{L}\right)-C_{i},
$$

where the coordination parameter $r$ is any positive number $(0<r)$. Instead of being a weight in a weighted average function, here $r$ expresses the relative perceived importance of coordination at an individual level through the way it compares to the implied coefficient of unity on the $-\left(a_{i}-\theta\right)^{2}$ term. Aggregating the above payoff yields:

$$
W^{\mathrm{MS}}=-\int_{0}^{1}\left(a_{i}-\theta\right)^{2} d i-\int_{0}^{1} C_{i} d i
$$

which no longer contains $r$. Thus, the coordination motive is neutral with respect to social welfare, but not the payoff function.

One potential issue with the expression in (3.1) is as follows. By varying the value of $r$, we change the importance of coordination relative not only to the loss from imperfect alignment of agent $i$ 's actions and $\theta$, but also relative to the information cost $C_{i}$, which technically also has a coefficient of unity. This creates the potential for perceived discounting of information cost from agent $i$ 's perspective, which, in turn, can lead to the existence of an added hidden mechanism in agent $i$ 's decision-making. In fact, we suspect this is the case in the linear information cost setup. Namely, as discussed in Section 3.5.2.5, optimally designed policy is unable to replicate efficient outcomes. This explanation is further supported by the fact that policy is able to replicate efficient outcomes in the linear cost case with the 
James-Lawler payoff function (see Section 3.6.2.5), the latter not suffering from the aforementioned vulnerability.

The original James and Lawler (2011) payoff function is given by:

$$
u_{i}^{\mathrm{JL}}=-\left(a_{i}-\left(1-r^{\mathrm{JL}}\right) \theta-r^{\mathrm{JL}} \bar{a}\right)^{2},
$$

where $0<r^{\mathrm{JL}}<1$ is the coordination coefficient, and $\bar{a}=\int_{0}^{1} a_{i} d i$. Expression (C.7) was constructed to be comparable with the original M-S payoff function in that agent $i$ 's best response in both cases is exactly the same. The distinction lies in the fact that, when the J-L payoff is aggregated, the coordination motive is not neutral:

$$
W^{\mathrm{JL}}=-\int_{0}^{1}\left(a_{i}-\left(1-r^{\mathrm{JL}}\right) \theta-r^{\mathrm{JL}} \bar{a}\right)^{2} .
$$

In fact, the true social benefit from coordination is higher than what is perceived by individual agents.

Adding a cost component to the payoff in (C.7) does not invalidate the key characteristic of the James-Lawler payoff function, the way it did in the Morris-Shin case. However, as mentioned above, (C.1) and (C.7) are comparable in that they imply identical expressions for agent $i$ 's best response. Re-formulation of the MorrisShin payoff leads to a change in the best response expression, thus, the James-Lawler payoff requires re-formulation in order to maintain the comparability. As a result, we re-formulate the original James-Lawler payoff function in the way presented in $(3.2)$ :

$$
u_{i}^{\mathrm{JL}}=-\left[a_{i}-\theta+r\left(a_{i}-\bar{a}\right)\right]^{2}-C_{i},
$$

where the coordination parameter $r$ is any positive number $(0<r)$. The added benefit of the re-formulation is consistency in notation with respect to the coordination parameter $r$.

\section{C.2 Model: deriving the degrees of coordination}

In order to derive the equilibrium and collectively efficient degrees of coordination, or EDoC and CEDoC, respectively, for re-formulated M-S and J-L payoff functions, we use the definitions provided in Angeletos and Pavan (2007a). EDoC is the slope of agent $i$ 's best response with respect to aggregate action in equilibrium, while CEDoC is the slope of agent $i$ 's best response with respect to aggregate action in an efficient benchmark scenario. 
We begin with the M-S payoff, from (3.1):

$$
u_{i}^{\mathrm{MS}}=-\left(a_{i}-\theta\right)^{2}-r\left(L_{i}-\bar{L}\right)-C_{i} .
$$

To find agent $i$ 's best response, we maximise the payoff function with respect to agent's $i$ action $a_{i}$.

$$
a_{i}=\frac{1}{1+r} \theta+\frac{r}{1+r} \bar{a}
$$

The coefficient on the average action $\bar{a}$ is then the equilibrium degree of coordination:

$$
E D o C=\frac{r}{1+r}
$$

which is monotonically increasing in the coordination parameter $r$. Since, by its design, the J-L payoff function yields the same best-response expression, the EDoC in (C.10) is the same for both payoff functions.

Next, we construct an efficient benchmark solution for the M-S payoff function. The efficient benchmark cases are discussed in more detail in the main text of this Chapter, thus, here we only present a brief derivation procedure, drawing on various assumptions in Section 3.2. In order to identify agent $i$ 's efficient response, we first need to obtain the social welfare expression by aggregating the payoff across the population:

$$
W^{\mathrm{MS}}=-\int_{0}^{1} u_{i}^{\mathrm{MS}} d i=-\int_{0}^{1}\left(a_{i}-\theta\right)^{2} d i-\int_{0}^{1} C_{i} d i .
$$

We assume full CBT and that individual action is a linear function of two signals:

$$
a_{i}=\kappa_{1} x_{i}+\kappa_{2} z
$$

where the signals are defined as follows:

$$
\begin{gathered}
x_{i}=\theta+\varepsilon_{i} \\
z=\theta+\phi .
\end{gathered}
$$

We substitute the above expressions into social welfare:

$$
W=-\int_{0}^{1}\left[\kappa_{1}\left(\theta+\varepsilon_{i}\right)+\kappa_{2}(\theta+\phi)-\theta\right]^{2} d i-\int_{0}^{1} C_{i} d i
$$

In the efficient scenario, the central bank is assumed to be able to set $\kappa_{1}$ and $\kappa_{2}$ or, in other words, directly induce a collectively efficient response by individual agents. To find the efficient values for $\kappa_{1}$ and $\kappa_{2}$, we take unconditional expectations of the 
welfare expression and maximise the result with respect to the weights:

$$
\max E[W]=-\frac{\kappa_{1}^{2}}{\beta}-\frac{\kappa_{2}^{2}}{\gamma}-\left(1-\kappa_{1}-\kappa_{2}\right)^{2} \sigma_{\theta}^{2}-\int_{0}^{1} C_{i} d i \text { w.r.t. } \kappa_{1}, \kappa_{2},
$$

where we restrict $\kappa_{1}+\kappa_{2}=1$. We find agent $i$ 's collectively efficient response to be:

$$
\tilde{a}_{i}=\frac{\beta}{\beta+\gamma} \cdot x_{i}+\frac{\gamma}{\beta+\gamma} \cdot z
$$

where $\tilde{\kappa}_{1}=\frac{\beta}{\beta+\gamma}$ and $\tilde{\kappa}_{2}=\frac{\gamma}{\beta+\gamma}$. We conjecture that socially efficient action can also be expressed as a linear function of agent $i$ 's expectation of the state variable $\theta$ and the average action $\bar{a}$ :

$$
\tilde{a}_{i}=\omega_{1} E_{i}[\theta]+\omega_{2} E_{i}[\tilde{\bar{a}}],
$$

where $E_{i}[\cdot]$ is the expectations conditional on signals $x_{i}$ and $z$ and $\widetilde{\bar{a}}$ is the socially optimal average action. In (C.16) $\omega_{2}$ is effectively the CEDoC that we are ultimately looking for. Assuming that $\varepsilon_{j}$ has a zero mean, average action and agent $i$ 's expectation thereof are given by:

$$
\begin{aligned}
& \tilde{\bar{a}}=\int_{0}^{1} \tilde{a}_{j} d j=\int_{0}^{1} \tilde{\kappa}_{1} x_{j}+\tilde{\kappa}_{2} z d j=\tilde{\kappa_{1}} \theta+\tilde{\kappa_{2}} z \\
& E_{i}[\tilde{\bar{a}}]=\tilde{\kappa_{1}} E_{i}[\theta]+\tilde{\kappa_{2}} z .
\end{aligned}
$$

We assume that the expected value of $\theta$ is:

$$
E_{i}[\theta]=\frac{\beta x_{i}+\gamma z}{\beta+\gamma}
$$

Finally, we construct a system of equations from the two expressions for socially optimal action in (C.15) and (C.16) with appropriate substitutions:

$$
\begin{aligned}
& \tilde{a}_{i}=\frac{\beta}{\beta+\gamma} \cdot x_{i}+\frac{\gamma}{\beta+\gamma} \cdot z \\
& \tilde{a}_{i}=\omega_{1} \cdot \frac{\beta x_{i}+\gamma z}{\beta+\gamma}+\omega_{2}\left(\tilde{\kappa_{1}} \cdot \frac{\beta x_{i}+\gamma z}{\beta+\gamma}+\tilde{\kappa_{2}} z\right) .
\end{aligned}
$$

By comparing the coefficients on $x_{i}$ and $z$, we obtain an expression for $\omega_{2}$ :

$$
C E D o C^{\mathrm{MS}}=0
$$

A CEDoC equal to zero is consistent with the characteristics of the M-S payoff function, in that $C E D o C^{\mathrm{MS}}<E D o C$ for all $0<r$. The vertical distance between $C E D o C^{\mathrm{MS}}$ and $E D o C$ is monotonically increasing in $r$ for $0<r$. For a visual representation, see Figure C.1. 


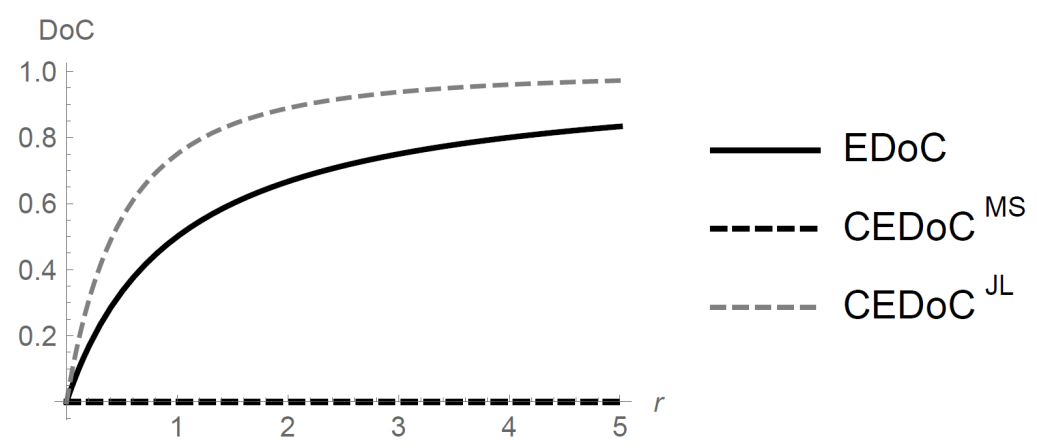

Figure C.1: Model - Degrees of Coordination (M-S and J-L)

To find the CEDoC for the J-L case, we need to repeat the efficient benchmark scenario derivations using the J-L payoff function. Social welfare is given by:

$$
\begin{aligned}
W^{\mathrm{JL}}= & -\int_{0}^{1} u_{i}^{\mathrm{JL}} d i=-\int_{0}^{1}\left[a_{i}-\theta+r\left(a_{i}-\bar{a}\right)\right]^{2} d i-\int_{0}^{1} C_{i} d i \\
W= & -\int_{0}^{1}\left\{\kappa_{1}\left(\theta+\varepsilon_{i}\right)+\kappa_{2}(\theta+\phi)-\theta+r\left[\kappa_{1}\left(\theta+\varepsilon_{i}\right)+\kappa_{2}(\theta+\phi)-\kappa_{1} \theta-\right.\right. \\
& \left.\left.-\kappa_{2}(\theta+\phi)\right]\right\}^{2} d i-\int_{0}^{1} C_{i} d i .
\end{aligned}
$$

Taking unconditional expectations of the above yields:

$$
E[W]=-\frac{(1+r)^{2} \kappa_{1}^{2}}{\beta}-\frac{\kappa_{2}^{2}}{\gamma}-\left(1-\kappa_{1}-\kappa_{2}\right)^{2} \sigma_{\theta}^{2}-\int_{0}^{1} C_{i} d i
$$

Maximising expected social welfare with respect to $\kappa_{1}$ and $\kappa_{2}$ and subject to $\kappa_{1}+\kappa_{2}=$ 1, we obtain the collectively efficient response:

$$
\tilde{a}_{i}=\frac{\beta}{\beta+(1+r)^{2} \gamma} \cdot x_{i}+\frac{\gamma}{\beta+(1+r)^{2} \gamma} \cdot z
$$

where $\tilde{\kappa}_{1}=\frac{\beta}{\beta+(1+r)^{2} \gamma}$ and $\tilde{\kappa}_{2}=\frac{\gamma}{\beta+(1+r)^{2} \gamma}$. Comparing it with the alternative formulation from (C.16) and solving for $\omega_{2}$ yields:

$$
C E D o C^{\mathrm{JL}}=\frac{(2+r) r}{(1+r)^{2}}
$$

In line with the characteristics of the original J-L payoff function, $E D o C<C E D o C^{\mathrm{JL}}$ for all $0<r$. In the J-L case, the CEDoC is strictly positive and monotonically increasing in $r$. As Figure C.1 demonstrates, the vertical distance between $E D o C$ and $C E D o C^{\mathrm{JL}}$ is increasing in $r$ for $0<r<1$ and decreasing in $r$ for $1<r$, reaching 
it's maximum at $r=1$.

\section{C.3 Model: volatility and dispersion of actions}

Angeletos and Pavan (2007a) introduce the terms volatility and dispersion when discussing different components of social welfare and agents' payoffs.

Volatility is defined as the common variation in actions due to noise in public information. Algebraically, volatility is defined as variance of the difference between average action and action profile of the population under perfect information. In the coordination games presented in this Chapter, under full transparency, agents choose their action to be equal to the state variable $\theta$. Thus, volatility can be expressed the following way:

$$
\text { Volatility }=\operatorname{Var}[\bar{a}-\theta]
$$

Given that in a P-NE average action is $\bar{a}^{P}=\kappa_{1}^{P} \theta+\kappa_{2}^{P} y$, where $\kappa_{1}^{P}+\kappa_{2}^{P}=1$ and $y=\theta+\phi+\eta$ :

$$
\operatorname{Var}\left[\bar{a}^{P}-\theta\right]=\operatorname{Var}\left[\kappa_{2}^{P}(\phi+\eta)\right]=E\left[\left(\kappa_{2}^{P}\right)^{2}(\phi+\eta)^{2}\right]-\left(E\left[\kappa_{2}^{P}(\phi+\eta)\right]\right)^{2}
$$

where the second term is equal to zero. Taking expectation then yields:

$$
\operatorname{Volatility}^{P}=\frac{\left(\kappa_{2}^{P}\right)^{2}}{\alpha}
$$

Given that in a N-NE the average action is just $\bar{a}^{N}=y$, volatility is:

$$
\operatorname{Volatility}^{N}=\frac{1}{\alpha}
$$

In contrast to volatility, dispersion is defined as idiosyncratic variation in actions due to noise in private information. Algebraically, this translates to the variance of difference between agent $i$ 's action and the average action across population:

$$
\text { Dispersion }=\operatorname{Var}\left[a_{i}-\bar{a}\right]
$$

Given that in a P-NE agent $i$ 's action is given by $a_{i}^{P}=\kappa_{1}^{P} x_{i}+\kappa_{2}^{P} y$, simplifying the 
above expression yields:

$$
\operatorname{Var}\left[a_{i}^{P}-\bar{a}^{P}\right]=\operatorname{Var}\left[\kappa_{1}^{P} \varepsilon_{i}\right]=E\left[\left(\kappa_{1}^{P}\right)^{2} \varepsilon_{i}^{2}\right]-\left(E\left[\kappa_{1}^{P} \varepsilon_{i}\right]\right)^{2},
$$

where the second terms is equal to zero. Taking expectations of the above:

$$
\operatorname{Dispersion}^{P}=\frac{\left(\kappa_{1}^{P}\right)^{2}}{\beta} \text {. }
$$

Considering that there is no private information in N-NE and $\kappa_{1}^{N}$ is essentially equal to zero, there is no dispersion in N-NE:

$$
\operatorname{Dispersion}^{N}=0
$$

Direct policy intervention aims to reduce volatility by using CB's private information to bring the economy closer to a perfect information scenario. By inserting instrument adjustment $g$ directly into (C.26), we can demonstrate the effect that policy has on volatility:

$$
\text { Volatility }=\operatorname{Var}[\bar{a}-\theta-g]
$$

Given that in a P-NE average action is $\bar{a}^{P}=\kappa_{1}^{P} \theta+\kappa_{2}^{P} y$, where $\kappa_{1}^{P}+\kappa_{2}^{P}=1+\rho$, $y=\theta+\phi+\eta$, as well as $g=\rho z$, and $z=\theta+\phi$ :

$\operatorname{Var}\left[\bar{a}^{P}-\theta-g\right]=\operatorname{Var}\left[\kappa_{2}^{P}(\phi+\eta)-\rho \phi\right]=E\left[\left(\kappa_{2}^{P}(\phi+\eta)-\rho \phi\right)^{2}\right]-\left(E\left[\kappa_{2}^{P}(\phi+\eta)-\rho \phi\right]\right)^{2}$

where the second term is equal to zero. Taking expectation then yields:

$$
\text { Volatility }^{P}=\frac{\left(\kappa_{2}^{P}\right)^{2}}{\alpha}-\frac{\left(2 \kappa_{2}^{P}-\rho\right) \rho}{\gamma}
$$

Thus, compared with the no-policy case in (C.27), volatility is reduced by the value of the second term in (C.33). Given that in a N-NE the average action is $\bar{a}^{N}=(1+\rho) y$, volatility is:

$$
\text { Volatility }^{N}=\frac{(1+\rho)^{2}}{\alpha}-\frac{(2+\rho) \rho}{\gamma} .
$$

It should be noted that instrument adjustment also has an indirect effect on dispersion and volatility in P-NE, which works through the best-response weights $\kappa_{1}^{P}$ and $\kappa_{2}^{P}$.

Using the above definitions, expected welfare can be conveniently split into three components - loss from volatility, loss from dispersion of actions, and the cost of 
private information acquisition. The M-S welfare expression from (3.38) can be re-expressed by adding and subtracting the average action:

$$
\begin{aligned}
W^{\mathrm{MS}} & =\int_{0}^{1}-\left(a_{i}-\bar{a}+\bar{a}-\theta-g\right)^{2}-C_{i} d i= \\
& =\int_{0}^{1}-\left(a_{i}-\bar{a}\right)^{2}-2\left(a_{i}-\bar{a}\right)(\bar{a}-\theta-g)-(\bar{a}-\theta-g)^{2}-C_{i} d i= \\
& =-\int_{0}^{1}\left(a_{i}-\bar{a}\right)^{2} d i-2(\bar{a}-\theta-g) \int_{0}^{1}\left(a_{i}-\bar{a}\right) d i-\int_{0}^{1}(\bar{a}-\theta-g)^{2} d i-\int_{0}^{1} C_{i} d i
\end{aligned}
$$

where:

$$
-2(\bar{a}-\theta-g) \int_{0}^{1}\left(a_{i}-\bar{a}\right) d i=-2(\bar{a}-\theta-g)(\bar{a}-\bar{a})=0 .
$$

Thus:

$$
W^{\mathrm{MS}}=-\int_{0}^{1}\left(a_{i}-\bar{a}\right)^{2} d i-\int_{0}^{1}(\bar{a}-\theta-g)^{2} d i-\int_{0}^{1} C_{i} d i .
$$

For a P-NE, substituting for $a_{i}^{P}, \bar{a}^{P}, g, x_{i}, y$, and $z$ and taking expectations of the resulting expression yields:

$$
E\left[W^{\mathrm{MS}-\mathrm{P}}\right]=-\frac{\left(\kappa_{1}^{P}\right)^{2}}{\beta}-\left[\frac{\left(\kappa_{2}^{P}\right)^{2}}{\alpha}-\frac{\left(2 \kappa_{2}-\rho\right) \rho}{\gamma}\right]-\int_{0}^{1} C_{i} d i
$$

where the three components (second component being the middle two terms in the square brackets) correspond to:

$$
E\left[W^{\mathrm{MS}-\mathrm{P}}\right]=- \text { Dispersion }^{P}-\left[\text { Volatility }^{P}\right]-\text { Information Cost. }
$$

Adding and subtracting $\bar{a}$ allows to express the J-L welfare function as:

$$
W^{\mathrm{JL}}=-(1+r)^{2} \int_{0}^{1}\left(a_{i}-\bar{a}\right)^{2} d i-\int_{0}^{1}(\bar{a}-\theta-g)^{2} d i-\int_{0}^{1} C_{i} d i .
$$

Using P-NE expressions for $a_{i}^{P}, \bar{a}^{P}, g, x_{i}, y$, and $z$ and taking expectations yields:

$$
E\left[W^{\mathrm{JL}-\mathrm{P}}\right]=-\frac{(1+r)^{2}\left(\kappa_{1}^{P}\right)^{2}}{\beta}-\left[\frac{\left(\kappa_{2}^{P}\right)^{2}}{\alpha}-\frac{\left(2 \kappa_{2}-\rho\right) \rho}{\gamma}\right]-\int_{0}^{1} C_{i} d i
$$

Thus, the J-L expected welfare in a P-NE corresponds to:

$$
E\left[W^{\mathrm{JL}-\mathrm{P}}\right]=-(1+r)^{2} \cdot \text { Dispersion }^{P}-\left[\text { Volatility }^{P}\right]-\text { Information Cost. }^{-}
$$

The fact that coordination is socially beneficial in the J-L setup leads to the disper- 
sion loss being amplified by $(1+r)^{2}$, which is the only difference compared to the $\mathrm{M}-\mathrm{S}$ expected welfare in $\mathrm{P}-\mathrm{NE}$.

The N-NE expected welfare outcome in M-S and J-L cases is identical:

$$
E\left[W^{N}\right]=-\frac{(1+\rho)^{2}}{\alpha}-\frac{(2+\rho) \rho}{\gamma}=- \text { Volatility }^{N},
$$

where the volatility expression is taken from (C.34).

\section{C.4 Fixed cost: M-S payoff function, no policy - efficiency of equilibria}

In order to comment on the efficiency of equilibria, we compare the different equilibrium cases with the efficient benchmark. We discuss efficiency with respect to three aspects: (i) information acquisition, (ii) information use, and (iii) expected welfare outcome.

In order to see if information acquisition is efficient, we compare the efficient critical price, from (3.78):

$$
\tilde{p}=\frac{\beta}{\gamma(\beta+\gamma)},
$$

with the appropriate values of $p_{\max }^{P}$ and $p_{\min }^{N}$. The intersection of $0<p \leq \tilde{p}$ and $0<p \leq p_{\max }^{P}$ corresponds to agents purchasing private information when it is efficient to do so. Similarly, the intersection of $\tilde{p} \leq p$ and $p_{m i n}^{N} \leq p$ corresponds to agents foregoing information acquisition when it is efficient not to purchase $x_{i}$.

To comment on information use, we look at the weights that agents place on the two signals in their best response. The efficient weights are as follows, from (3.75):

$$
\begin{aligned}
& \tilde{\kappa}_{1}^{P}=\frac{\beta}{\beta+\gamma} \\
& \tilde{\kappa}_{2}^{P}=1-\tilde{\kappa}_{1}^{P} \\
& \tilde{\kappa}_{2}^{N}=1 .
\end{aligned}
$$

Comparing the above expressions to the equilibrium weights demonstrates the effect that coordination has on the use of information. 
Finally, the expected welfare benchmark is, from (3.76) and (3.77):

$$
\begin{aligned}
& E\left[\tilde{W}^{P}\right]=-\frac{1}{\beta+\gamma}-p \\
& E\left[\tilde{W}^{N}\right]=-\frac{1}{\gamma}
\end{aligned}
$$

for a purchasing and a non-purchasing population, respectively.

Cases A and D: $(0<r \leq 1) \vee\left[(1<r) \wedge\left(\gamma_{1}<\gamma\right)\right]$, where $\gamma_{1}=\frac{(r-1) \beta}{1+r}$

In equilibrium, the $\mathrm{CB}$ commits to full $\mathrm{CBT}$ for all values of $p$. A range of prices exists $\left(p_{\min }^{N}<p<p_{\max }^{P}\right.$ ) for which both types of NE may exist. The relevant expressions are as follows, from (3.32), (3.60), (3.62), (3.69), and (3.46):

$$
\begin{aligned}
& E\left[W^{P}\right]_{\alpha=\gamma}=-\frac{(1+r)^{2} \gamma+\beta}{[(1+r) \gamma+\beta]^{2}}-p \\
& E\left[W^{N}\right]_{\alpha=\gamma}=-\frac{1}{\gamma} \\
& p_{\text {min }}^{N}=\frac{\beta}{(1+r) \gamma(\gamma+\beta)} \\
& p_{\text {max }}^{P}=\frac{(1+r) \beta(\gamma+\beta)}{\gamma[(1+r) \gamma+\beta]^{2}} \\
& \kappa_{1}^{P}=\frac{\beta}{(1+r) \gamma+\beta} \\
& \kappa_{2}^{P}=1-\kappa_{1}^{P} \\
& \kappa_{2}^{N}=1 .
\end{aligned}
$$

The assessment of efficiency yields results that are best presented introducing a further condition on parameter values. If $\gamma<\frac{\beta \sqrt{1+r}}{1+r}$ (and other conditions for Cases $\mathrm{A}$ and $\mathrm{D})$, the following aspects are efficient:

\begin{tabular}{clll} 
Condition & IA & IU & EWO \\
\hline & & & \\
$0<p<p_{\text {min }}^{N}$ & Yes (P-NE) & No (P-NE) & No (P-NE) \\
$p_{\text {min }}^{N} \leq p<\tilde{p}$ & Yes (if P-NE), No (if N-NE) & No (if P-NE) & No (if P-NE) \\
$\tilde{p}<p \leq p_{\max }^{P}$ & No (if P-NE), Yes (if N-NE) & Yes (if N-NE) & Yes (if N-NE) \\
$p_{\text {max }}^{P}<p$ & Yes (N-NE) & Yes (N-NE) & Yes (N-NE)
\end{tabular}


where $I A$ stands for information acquisition, IU for information use, and EWO for expected welfare outcome. If $\frac{\beta \sqrt{1+r}}{1+r}<\gamma$ (and other conditions for Cases A and $\mathrm{D})$, the following aspects are efficient:

\begin{tabular}{clll} 
Condition & IA & IU & EWO \\
\hline & & & \\
$0<p<p_{\text {min }}^{N}$ & Yes $(\mathrm{P}-\mathrm{NE})$ & No $(\mathrm{P}-\mathrm{NE})$ & No $(\mathrm{P}-\mathrm{NE})$ \\
$p_{\min }^{N} \leq p<p_{\max }^{P}$ & Yes (if P-NE), No (if N-NE) & No (if P-NE) & No (if P-NE) \\
$p_{\max }^{P} \leq p<\tilde{p}$ & No (N-NE) & - & - \\
$\tilde{p}<p$ & Yes $(\mathrm{N}-\mathrm{NE})$ & Yes $(\mathrm{N}-\mathrm{NE})$ & Yes $(\mathrm{N}-\mathrm{NE})$
\end{tabular}

Case B: $(1<r) \wedge\left(0<\gamma<\gamma_{1}\right)$, where $\gamma_{1}=\frac{(r-1) \beta}{1+r}$

For higher values of $r$ (i.e. $r_{1}<r$ ), in equilibrium the $\mathrm{CB}$ commits to zero CBT for $0<p<p_{\text {dom }}$ and full CBT otherwise. For lower value of the coordination parameter $\left(0<r<r_{1}\right)^{1}$, both types of equilibria exist for $p_{\text {dom }} \leq p<p_{\max }^{P}$. The relevant expressions are as follows, from (3.32), (3.60), (3.61), (3.62), and (3.46):

$$
\begin{aligned}
& E\left[W^{P}\right]_{\alpha=\gamma}=-\frac{(1+r)^{2} \gamma+\beta}{[(1+r) \gamma+\beta]^{2}}-p \\
& E\left[W^{P}\right]_{\alpha=0}=-\frac{1}{\beta}-p \\
& E\left[W^{N}\right]_{\alpha=\gamma}=-\frac{1}{\gamma} \\
& p_{\text {dom }}=\frac{1}{\gamma-\frac{1}{\beta}} \\
& p_{\text {max }}^{P}=\frac{(1+r) \beta(\gamma+\beta)}{\gamma[(1+r) \gamma+\beta]^{2}} \\
& \left.\kappa_{1}^{P}\right|_{\alpha=0}=1 \\
& \left.\kappa_{2}^{P}\right|_{\alpha=0}=0 \\
& \kappa_{2}^{N}=1 .
\end{aligned}
$$

${ }^{1}$ From $(3.70), r_{1} \equiv \frac{1}{2(\beta-\gamma) \gamma^{2}}\left[\beta^{3}-\beta^{2} \gamma+2 \gamma^{3}+\beta \sqrt{\beta(\beta+\gamma)\left(\beta^{2}-3 \beta \gamma+4 \gamma^{2}\right)}\right]$. 
If $r_{1}<r$ (and other conditions for Case B), the following aspects are efficient:

\begin{tabular}{clll} 
Condition & IA & IU & EWO \\
\hline & & & \\
$0<p<p_{\text {dom }}$ & Yes $(\mathrm{P}-\mathrm{NE})$ & No $(\mathrm{P}-\mathrm{NE})$ & No $(\mathrm{P}-\mathrm{NE})$ \\
$p_{\text {dom }} \leq p<\tilde{p}$ & No $(\mathrm{N}-\mathrm{NE})$ & - & - \\
$\tilde{p}<p$ & Yes $(\mathrm{N}-\mathrm{NE})$ & Yes $(\mathrm{N}-\mathrm{NE})$ & Yes $(\mathrm{N}-\mathrm{NE})$
\end{tabular}

If $\left(\gamma<\frac{\beta \sqrt{1+r}}{1+r}\right) \wedge\left(\frac{\beta+\gamma}{\beta-\gamma}<r \leq r_{1}\right)$ (and other conditions for Case B), the following aspects are efficient:

\begin{tabular}{clll} 
Condition & IA & IU & EWO \\
\hline & & & \\
$0<p<p_{\text {dom }}$ & Yes (P-NE) & No (P-NE) & No (P-NE) \\
$p_{\text {dom }} \leq p<\tilde{p}$ & Yes (if P-NE), No (if N-NE) & No (if P-NE) & No (if P-NE) \\
$\tilde{p}<p \leq p_{\max }^{P}$ & No (if P-NE), Yes (if N-NE) & Yes (if N-NE) & Yes (if N-NE) \\
$p_{\max }^{P}<p$ & Yes (N-NE) & Yes (N-NE) & Yes (N-NE)
\end{tabular}

If $\left(\frac{\beta \sqrt{1+r}}{1+r}<\gamma\right) \wedge\left(\frac{\beta+\gamma}{\beta-\gamma}<r \leq r_{1}\right)$ (and other conditions for Case B), the following aspects are efficient:

\begin{tabular}{clll} 
Condition & IA & IU & EWO \\
\hline & & & \\
$0<p<p_{\text {dom }}$ & Yes $(\mathrm{P}-\mathrm{NE})$ & No $(\mathrm{P}-\mathrm{NE})$ & No $(\mathrm{P}-\mathrm{NE})$ \\
$p_{\text {dom }} \leq p<p_{\max }^{P}$ & Yes $($ if $\mathrm{P}-\mathrm{NE})$, No (if N-NE) & No (if P-NE) & No (if P-NE) \\
$p_{\max }^{P} \leq p<\tilde{p}$ & No $(\mathrm{N}-\mathrm{NE})$ & - & - \\
$\tilde{p}<p$ & Yes $(\mathrm{N}-\mathrm{NE})$ & Yes $(\mathrm{N}-\mathrm{NE})$ & Yes $(\mathrm{N}-\mathrm{NE})$
\end{tabular}

Case C: $(1<r) \wedge\left(\gamma=\gamma_{1}\right)$, where $\gamma_{1}=\frac{(r-1) \beta}{1+r}$

As in Case B, in equilibrium the CB commits to zero CBT for $0<p<p_{\text {dom }}$ and full CBT for $p_{\max }^{P}<p$. Both types of equilibria exist for $p_{\text {dom }} \leq p<p_{\max }^{P}$. The 
relevant expressions are as follows, from (3.71), (3.73), and (3.74):

$$
\begin{aligned}
& E\left[W^{P}\right]_{\alpha=0, \gamma=\gamma_{1}}=E\left[W^{P}\right]_{\alpha=\gamma=\gamma_{1}}=-\frac{1}{\beta}-p \\
& E\left[W^{N}\right]_{\alpha=\gamma=\gamma_{1}}=-\frac{1+r}{(r-1) \beta} \\
& \left.p_{\text {dom }}\right|_{\gamma=\gamma_{1}}=\frac{2}{(r-1) \beta} \\
& \left.p_{\text {max }}^{P}\right|_{\alpha=\gamma=\gamma_{1}}=\frac{2(1+r)}{(r-1) r \beta} \\
& \left.\kappa_{1}^{P}\right|_{\alpha=0}=1 \\
& \left.\kappa_{2}^{P}\right|_{\alpha=0}=0 \\
& \kappa_{2}^{N}=1 .
\end{aligned}
$$

If $1<r<3$ (and other conditions for Case C), the following aspects are efficient:

\begin{tabular}{clll} 
Condition & IA & IU & EWO \\
\hline & & & \\
$0<p<p_{\text {dom }}$ & Yes (P-NE) & No (P-NE) & No (P-NE) \\
$p_{\text {dom }} \leq p<\tilde{p}$ & Yes (if P-NE), No (if N-NE) & No (if P-NE) & No (if P-NE) \\
$\tilde{p}<p \leq p_{\text {max }}^{P}$ & No (if P-NE), Yes (if N-NE) & Yes (if N-NE) & Yes (if N-NE) \\
$p_{\max }^{P}<p$ & Yes (N-NE) & Yes (N-NE) & Yes (N-NE)
\end{tabular}

If $3<r$ (and other conditions for Case C), the following aspects are efficient:

\begin{tabular}{clll} 
Condition & IA & IU & EWO \\
\hline & & & \\
$0<p<p_{\text {dom }}$ & Yes $(\mathrm{P}-\mathrm{NE})$ & No $(\mathrm{P}-\mathrm{NE})$ & No $(\mathrm{P}-\mathrm{NE})$ \\
$p_{\text {dom }} \leq p<p_{\max }^{P}$ & Yes (if P-NE), No (if N-NE) & No (if P-NE) & No (if P-NE) \\
$p_{\max }^{P} \leq p<\tilde{p}$ & No $(\mathrm{N}-\mathrm{NE})$ & - & - \\
$\tilde{p}<p$ & Yes $(\mathrm{N}-\mathrm{NE})$ & Yes $(\mathrm{N}-\mathrm{NE})$ & Yes $(\mathrm{N}-\mathrm{NE})$
\end{tabular}

\section{C.5 Linear cost: information accuracy and com- monality}

Angeletos and Pavan (2007a) introduce the terms accuracy and commonality to conveniently characterise available information.

Accuracy of available information is the precision of agents' forecasts about $\theta$ 
(i.e. the inverse of the variance of forecast errors about $\theta$ ). A purchasing agent $i$ 's forecast error is given by:

$$
\mathrm{FE}_{i}=\theta-E_{i}\left[\theta \mid x_{i}, y\right]
$$

where $E_{i}\left[\theta \mid x_{i}, y\right]=\frac{\alpha y+\beta_{i} x_{i}}{\alpha+\beta_{i}}$. The variance of the forecast error is then:

$$
\operatorname{Var}\left[\mathrm{FE}_{i}\right]=E\left[\left(\mathrm{FE}_{i}\right)^{2}\right]-\left(E\left[\mathrm{FE}_{i}\right]\right)^{2}
$$

Substituting for $x_{i}$ and $y$ and taking expectations yields:

$$
\operatorname{Var}\left[\mathrm{FE}_{i}\right]=\frac{1}{\alpha+\beta_{i}}
$$

It is more intuitive to discuss accuracy in terms of precision rather than variance, thus, we invert the above result:

$$
\operatorname{Accuracy}_{i}^{P}=\alpha+\beta_{i} \text {. }
$$

The above is accuracy of information available to a purchasing agent $i$. In a symmetric P-NE accuracy is the same for all agents:

$$
\operatorname{Accuracy}^{P}=\alpha+\beta \text {. }
$$

Following the same procedure it can be easily shown that accuracy of information in a N-NE is simply $\alpha$ :

$$
\operatorname{Accuracy}^{N}=\alpha
$$

Commonality of available information is the correlation of forecast errors across agents. Correlation between forecast errors of agent $i\left(\mathrm{FE}_{i}\right)$ and agent $j\left(\mathrm{FE}_{j}\right)$ is equal to the covariance between the two forecast errors divided by the product of standard deviations (square root of the non-inverted accuracies). Since the mean of both forecast errors is the same $\left(E\left[\mathrm{FE}_{i}\right]=E\left[\mathrm{FE}_{j}\right]=0\right)$, covariance is equal to the expectation of the product of the forecast errors.

$$
\begin{aligned}
\operatorname{corr}\left(\mathrm{FE}_{i}, \mathrm{FE}_{j}\right) & =\frac{\operatorname{cov}\left(\mathrm{FE}_{i}, \mathrm{FE}_{j}\right)}{\sqrt{\operatorname{Accuracy}_{i}^{-1}} \sqrt{\text { Accuracy }_{j}^{-1}}}= \\
& =\frac{E\left[\left(\mathrm{FE}_{i}-E\left[\mathrm{FE}_{i}\right]\right)\left(\mathrm{FE}_{j}-E\left[\mathrm{FE}_{j}\right]\right)\right]}{\sqrt{\text { Accuracy }_{i}^{-1}} \sqrt{\text { Accuracy }_{j}^{-1}}}= \\
& =\frac{E\left[\mathrm{FE}_{i} \cdot \mathrm{FE}_{j}\right]}{\sqrt{\text { Accuracy }_{i}^{-1}} \sqrt{\text { Accuracy }_{j}^{-1}}},
\end{aligned}
$$

where symmetry dictates that accuracy of agent $j$ 's information is the same for all 
$j \neq i$ (in other words $\beta_{j}=\beta$ ). The resulting expression for commonality is not agent-specific and in a $\mathrm{P}-\mathrm{NE}$ is equal to:

$$
\text { Commonality }^{P}=\frac{\alpha}{\alpha+\beta},
$$

where the value of commonality lies between 0 and 1 . In a $\mathrm{N}-\mathrm{NE}$, there is perfect correlation of forecast errors and commonality is equal to unity:

$$
\text { Commonality }^{N}=1 .
$$




\section{Appendix D}

\section{D.1 Agent $i$ 's forecast of instrument adjustment $g$ and its accuracy}

In order to comment on the effect that a change in $p, \alpha, \omega, \tau$, and $\beta$ have on agent $i$ 's ability to forecast the CB's instrument adjustment $g$, we need to derive (i) agent $i$ 's conditional expectation of $g\left(E_{i}\left[g \mid x_{i}, y\right]\right)$, as well as (ii) the accuracy of agent $i$ 's forecast of $g$.

Agent $i$ forms an expectation of the CB's instrument adjustment $g$ conditional on the observed signals $x_{i}$ and $y$. Thus, we begin by assuming that agent $i$ 's expectation of $g$ can be expressed as a linear combination of the two signals:

$$
E_{i}[g]=a x_{i}+b y
$$

where $a$ and $b$ are used here for convenience of notation only and must not be confused with any variables defined in the main sections of this Chapter. Next we need to find $a$ and $b$ so as to minimise the mean squared error (MSE) of $E_{i}[g]$ as an estimator of $g$ :

$$
\min \operatorname{MSE}\left[E_{i}[g]\right] \quad \text { w.r.t. } a \text { and } b \text {, }
$$

where the mean squared error is defined as:

$$
\operatorname{MSE}\left[E_{i}[g]\right]=E\left[g-E_{i}[g]\right]^{2} .
$$

We begin by substituting (D.1), the CB's rule from (4.2), average action from (4.13), 
and the definitions of the three signals:

$$
\begin{aligned}
g=\tau \bar{a}+(1-\tau) z & =\tau[\kappa \theta+(1-\kappa) y]+(1-\tau) z \\
x_{i} & =\theta+\varepsilon_{i} \\
y & =\theta+\eta \\
z & =\theta+\phi,
\end{aligned}
$$

into (D.3):

$$
\begin{aligned}
& \mathrm{MSE}=E\left[\tau \kappa \theta+\tau(1-\kappa) y+(1-\tau) z-a x_{i}-b y\right]^{2}= \\
& =E\left[(1-a-b) \theta+(1-\tau) \phi-a \varepsilon_{i}-(b-\tau+\tau \kappa) \eta\right]^{2} .
\end{aligned}
$$

We expand the expression in (D.5), take expectations and exclude summands that are equal to zero due to independence of noises:

$$
\operatorname{MSE}=(1-a-b)^{2} \sigma_{\theta}^{2}+\frac{a^{2}}{\beta}+\frac{(b-\tau+\tau \kappa)^{2}}{\alpha}+\frac{(1-\tau)^{2}}{\gamma}
$$

Considering that $\sigma_{\theta}^{2}$ is not defined (due to $\theta$ being drawn from an improper prior), we need to impose a restriction on $a$ and $b$ in order to ensure that the MSE is not undefined. Thus, we make an additional assumption that $a+b=1$. With this assumption in mind, we find the values of coefficients $a$ and $b$ that minimise the MSE in (D.6):

$$
\begin{aligned}
& a=\frac{(1-\tau+\tau \kappa) \beta}{\alpha+\beta} \\
& b=\frac{\alpha+(1-\kappa) \tau \beta}{\alpha+\beta} .
\end{aligned}
$$

By substituting (D.7a) and (D.7b) into equation (D.1) we arrive at the desired expression for agent $i$ 's conditional expectation of instrument adjustment $g$ :

$$
E_{i}[g]=\frac{(1-\tau+\tau \kappa) \beta x_{i}+[\alpha+(1-\kappa) \tau \beta] y}{\alpha+\beta} .
$$

Having arrived at $E_{i}[g]$, we are in the position to derive the expression for accuracy of $E_{i}[g]$. In order to do that, we first find the variance of agent $i$ 's forecast errors:

$$
\operatorname{Var}\left[g-E_{i}[g]\right]=E\left[\left(g-E_{i}[g]\right)^{2}\right]-\left(E\left[g-E_{i}[g]\right]\right)^{2}
$$

where the second term on the right-hand side is equal to zero. Substituting for $g$ 
and $E_{i}[g]$ and taking expectation of the resulting expression yields:

$$
\operatorname{Var}\left[g-E_{i}[g]\right]=\frac{(1-\tau+\tau \kappa)^{2}}{\alpha+\beta}+\frac{(1-\tau)^{2}}{\gamma}
$$

The inverse of the variance of forecast errors in (D.9) is the accuracy of agent $i$ 's forecast of $g$, which is given by:

$$
\operatorname{Accuracy}_{i}^{g}=\frac{(\alpha+\beta) \gamma}{(1-\tau)^{2}(\alpha+\beta)+(1-\tau+\tau \kappa)^{2} \gamma} \text {. }
$$

By substituting $\kappa=\kappa^{*}$ from (4.16) into (D.10), we get the accuracy of agent $i$ 's forecast of $g$ under the assumption of an exogenous $\beta$ :

$$
\left.\operatorname{Accuracy}_{i}^{g}\right|_{\kappa=\kappa^{*}}=\frac{(\alpha+\beta) \gamma[\alpha+\beta(1-\omega \tau)]^{2}}{(\alpha+\beta)(1-\tau)^{2}[\alpha+\beta(1-\omega \tau)]^{2}+[\alpha(1-\tau)+\beta(1-\omega \tau)]^{2} \gamma} .
$$

Expression (D.11) is strictly increasing in $\alpha, \omega$, and $\tau$, whereas the effect of a change in $\beta$ is ambiguous. On the one hand, higher $\beta$ allows the agent to better forecast the $\tau \kappa \theta$ and $(1-\tau) z$ components of the instrument adjustment; on the other hand, higher $\beta$ leads to a higher $\kappa$, which, in turn, reduces the importance of the observable component $\tau(1-\kappa) y$. Differentiating (D.11) with respect to $\beta$ and solving the firstorder condition yields four stationary points, three of which are negative for all parameter values. The remaining stationary point:

$$
\beta_{A 1}=\frac{\alpha[(3+\sqrt{9-8 \omega}) \tau-2]}{2(1-\omega \tau)}
$$

is positive for $\frac{2}{3+\sqrt{9-8 \omega}}<\tau$ and is, in fact, a minimum. Thus, the accuracy of agent $i$ 's forecast of $g$ is decreasing in $\beta$ for:

$$
\left\{\begin{array}{l}
\frac{2}{3+\sqrt{9-8 \omega}}<\tau \\
0<\beta<\beta_{A 1},
\end{array}\right.
$$

and increasing in $\beta$, otherwise. The term $\frac{2}{3+\sqrt{9-8 \omega}}$ lies in the interval $\left(\frac{1}{3}, \frac{1}{2}\right)$, depending on the value of $\omega$. This means that, for low values of the policy parameter $\left(0<\tau<\frac{1}{3}\right)$, the accuracy of agent $i$ 's forecast of $g$ is increasing in $\beta$ for all parameter values.

By substituting $\beta=\beta^{*}$ from (4.20) into (D.11), we get the accuracy of agent $i$ 's 
forecast of $g$ under the assumption of an endogenous $\beta$ :

$$
\begin{aligned}
& \left.\operatorname{Accuracy}_{i}^{g}\right|_{\kappa=\kappa^{*}, \beta=\beta^{*}}=\gamma(1-\omega \tau)[1-(1+\alpha \sqrt{p}) \omega \tau] \times \\
& \times \frac{1}{\alpha^{2} \gamma p \sqrt{p} \tau^{2}-2 \alpha \gamma p \tau(1-\omega \tau)+(1-\tau)^{2}(1-\omega \tau)^{2}-(1-\omega \tau)\left\{\left[\gamma+\alpha(1-\tau)^{2}\right] \omega \tau-\gamma\right\} \sqrt{p}} .
\end{aligned}
$$

For $0<p<p_{1}$ (where $p_{1}=\frac{(1-\omega \tau)^{2}}{\alpha^{2}}$ from (4.22)), expression (D.14) is strictly increasing in $\alpha$ and $\tau$, but the effect of a change in $\omega$ or $p$ is ambiguous. The relationship between (D.14) and $p$ in a way mirrors that between (D.11) and $\beta$. Differentiating (D.14) and solving the first-order condition yields three stationary points, two of which are outside the permissible range $0<p<p_{1}$ for all parameter values. The remaining stationary point:

$$
p_{A 1}=\frac{(9-4 \omega-3 \sqrt{9-8 \omega})(1-\omega \tau)^{2}}{8 \alpha^{2} \omega^{2} \tau^{2}}
$$

is within the permissible range for $\frac{2}{3+\sqrt{9-8 \omega}}<\tau$ and is, in fact, a minimum. Thus, the accuracy of agent $i$ 's forecast of $g$ is increasing in $p$ for:

$$
\left\{\begin{array}{l}
\frac{2}{3+\sqrt{9-8 \omega}}<\tau \\
p_{A 1}<p
\end{array}\right.
$$

and decreasing in $p$, otherwise. As noted above, the term $\frac{2}{3+\sqrt{9-8 \omega}}$ lies in the interval $\left(\frac{1}{3}, \frac{1}{2}\right)$, depending on the value of $\omega$. This means that, for low values of the policy parameter $\left(0<\tau<\frac{1}{3}\right)$, the accuracy of agent $i$ 's forecast of $g$ is decreasing in $p$ for all parameter values. The sign of the first derivative of (D.14) with respect to $\omega$ depends on parameter value combinations. The derivative is negative when any one of the following sets of conditions is met:

$$
\begin{aligned}
& \left\{\begin{array}{l}
0<\tau \leq \frac{1}{3} \\
0<p<p_{1},
\end{array}\right. \\
& \left\{\begin{array}{l}
\frac{1}{3}<\tau<\frac{3-\sqrt{9-8 \omega}}{4 \omega} \\
0<p<p_{1},
\end{array}\right.
\end{aligned}
$$




$$
\left\{\begin{array}{l}
\frac{1}{3}<\tau<\frac{1}{2} \\
0<\omega \leq \frac{3 \tau-1}{2 \tau^{2}} \\
0<p<\frac{(1-2 \tau)^{2}(1-\omega \tau)^{2}}{\alpha^{2} \tau^{2}(1-2 \omega \tau)^{2}}
\end{array}\right.
$$

and positive when any one of the following sets of conditions is met:

$$
\begin{aligned}
& \left\{\begin{array}{l}
\frac{1}{2}<\tau<1 \\
\omega=\frac{1-\tau}{2 \tau^{2}} \\
0<p<\frac{(1-3 \tau)^{2}}{4 \alpha^{2} \tau^{2}}
\end{array}\right. \\
& \left\{\begin{array}{l}
\frac{1}{2}<\tau<1 \\
\omega \neq \frac{1-\tau}{2 \tau^{2}} \\
0<p<p_{1}
\end{array}\right. \\
& \left\{\begin{array}{l}
\frac{1}{3}<\tau \leq \frac{1}{2} \\
0<\omega \leq \frac{3 \tau-1}{2 \tau^{2}} \\
\frac{(1-2 \tau)^{2}(1-\omega \tau)^{2}}{\alpha^{2} \tau^{2}(1-2 \omega \tau)^{2}}<p<p_{1} .
\end{array}\right.
\end{aligned}
$$

When $\beta$ becomes endogenous, the effect of an increase in $\omega$ on the accuracy of the forecast of $g$ goes from being strictly positive to ambiguous. This is due to $\omega$ having a secondary effect on the forecast through the endogenous $\beta$, which is decreasing in $\omega$.

\section{D.2 Agent $i$ 's expected payoff as dispersion and volatility}

In our discussion of agent $i$ 's expected payoff we use the approach introduced in the previous Chapter. Namely, following Angeletos and Pavan (2007b), we identify a dispersion loss and a volatility loss within the expression for agent $i$ 's expected payoff.

Decomposition of agent $i$ 's expected payoff performed in the present Chapter slightly differs from the analysis carried out by Angeletos and Pavan (2007b). Firstly, here we decompose agent $i$ 's expected payoff, whereas Angeletos and Pavan apply the dispersion/volatility analysis to an expected welfare function (where welfare is 
defined as the aggregate of individual payoffs). Secondly, the definition of volatility loss is broadened in the present scenario, compared to that used by Angeletos and Pavan. The reason for this is the payoff function that we borrow directly from Morris and Shin (2018), from (4.3):

$$
u_{i}=-\omega\left(a_{i}-g\right)^{2}-(1-\omega)\left(a_{i}-\theta\right)^{2}-p \beta_{i} .
$$

Here the payoff function includes two independent sources of loss - that from misalignment of agent $i$ 's action with $\theta$ and that from misalignment of action with instrument $g$. The payoff includes no explicit coordination term. This is in stark contrast with the payoff function used by Angeletos and Pavan:

$$
u_{i}=-\left(a_{i}-\theta\right)^{2}-r L_{i}
$$

Angeletos-Pavan payoff function does not include a policy term, but it does include an explicit coordination term $-r L_{i}$ that represents the loss from misalignment of agent $i$ 's action with those of others.

Angeletos and Pavan explain that imperfect information about $\theta$ induces two types of loss that would not be incurred in a perfect information scenario. Dispersion is defined as the variation between agent $i$ 's action and the average action:

$$
\text { Dispersion }=\operatorname{Var}\left[a_{i}-\bar{a}\right]
$$

whereas volatility is defined as the variation between the equilibrium average action and the average action under perfect information:

$$
\text { Volatility }=\operatorname{Var}[\bar{a}-\theta]
$$

The former is associated with the idiosyncratic noise in private information, whereas the latter is related to the common noise in the public signal.

In the Angeletos-Pavan scenario, under perfect information, agents choose $a_{i}=$ $\bar{a}=\theta$ thereby eliminating dispersion and volatility (as well as ensuring perfect coordination), which, in turn, reduces expected payoff to zero. Identical reasoning could be applied to the present scenario if $\omega=0$ and agent $i$ 's sole aim was to match the fundamental. However, for $0<\omega<1$, agent $i$ also aims to match the instrument $g$, thus, the Angeletos-Pavan approach needs to be expanded to include the CB's objective. Namely, CB's only source of loss is the misalignment of $\theta$ and $g$, and commitment to an instrument rule leads to agent $i$ 's payoff becoming indirectly 
contingent on this misalignment. Under imperfect information, the decomposition of agent $i$ 's expected payoff partially reflects the misalignment of fundamental and the instrument. As will be demonstrated algebraically below, the term $-\omega\left(a_{i}-\right.$ $g)^{2}$ indirectly contributes to dispersion and volatility loss terms. Under perfect information, the CB's instrument adjustment would be equal to the state variable $(g=\theta)$, agent $i$ 's aim of matching the instrument would coincide with matching $\theta$, and, thus, agent $i$ 's payoff would collapse to:

$$
u_{i}=-\left(a_{i}-\theta\right)^{2}-p \beta_{i}
$$

Individual agents would have no need to purchase private information $\left(p \beta_{i}=0\right)$ and would find it optimal to choose $a_{i}=\bar{a}=\theta$. The perfect information outcome is clearly analogous to that of Angeletos and Pavan.

Having substituted CB's rule from (4.2) into agent $i$ 's payoff in (4.3), we get:

$$
u_{i}=-\omega\left[a_{i}-\tau \bar{a}-(1-\tau) z\right]^{2}-(1-\omega)\left(a_{i}-\theta\right)^{2}-p \beta_{i} .
$$

The term in the square brackets demonstrates how agent $i$ 's payoff depends on the alignment of agent $i$ 's action with the average action and CB's private information through the CB's rule. In order to facilitate the splitting of the payoff into convenient terms, we add and subtract $\bar{a}$ from the expressions in the first and the second squared terms:

$$
\begin{aligned}
u_{i}= & -\omega\left[a_{i}-\bar{a}+\bar{a}-\tau \bar{a}-(1-\tau) z\right]^{2}- \\
& -(1-\omega)\left(a_{i}-\bar{a}+\bar{a}-\theta\right)^{2}-p \beta_{i} .
\end{aligned}
$$

In this model, agent $i$ does not observe $z$, thus, agent $i$ 's expectation of $z$ and $\theta$ coincide $\left(E_{i}[z]=E_{i}[\theta]\right)$. In other words, the aim of matching $z$ can be interpreted as equivalent to aligning the action with the state variable. We can isolate a further component of volatility loss by expressing signal $z$ as $\theta+\phi$ :

$$
\begin{aligned}
u_{i}= & -\omega\left[\left(a_{i}-\bar{a}\right)+(1-\tau)(\bar{a}-\theta)-(1-\tau) \phi\right]^{2}- \\
& -(1-\omega)\left[\left(a_{i}-\bar{a}\right)+(\bar{a}-\theta)\right]^{2}-p \beta_{i} .
\end{aligned}
$$

Expanding the squared terms and rearranging the resulting expression yields:

$$
\begin{aligned}
u_{i}= & -\left(a_{i}-\bar{a}\right)^{2}-\left(1-2 \omega \tau+\omega \tau^{2}\right)(\bar{a}-\theta)^{2}+(1-\tau)^{2}(2 \bar{a}-2 \theta-\phi) \omega \phi+ \\
& +2\left(a_{i}-\bar{a}\right)[\theta-\omega \tau \theta+(1-\tau) \omega \phi-(1-\omega \tau) \bar{a}]-p \beta_{i} .
\end{aligned}
$$


We substitute for $a_{i}$ and $\bar{a}$ from (4.5) and (4.13), respectively, then we substitute definitions of $x_{i}$ and $y^{1}$, and take unconditional expectations of the resulting expression:

$$
\begin{aligned}
E\left[u_{i}\right]= & -\left[\frac{\left(\kappa-\kappa_{i}\right)^{2}}{\alpha}+\frac{\kappa_{i}^{2}}{\beta_{i}}\right]-\left[\frac{\left(1-2 \omega \tau+\omega \tau^{2}\right)(1-\kappa)^{2}}{\alpha}\right]- \\
& -\left[\frac{\omega(1-\tau)^{2}}{\gamma}+\frac{2(1-\kappa)\left(\kappa-\kappa_{i}\right)(1-\omega \tau)}{\alpha}\right]-p \beta_{i} .
\end{aligned}
$$

In a symmetric equilibrium, $\beta_{i}=\beta$ and $\kappa_{i}=\kappa$, thus, the above expression becomes:

$$
E\left[u_{i}\right]=-\frac{\kappa^{2}}{\beta}-\frac{\left(1-2 \omega \tau+\omega \tau^{2}\right)(1-\kappa)^{2}}{\alpha}-\frac{\omega(1-\tau)^{2}}{\gamma}-p \beta,
$$

where the terms correspond to:

$E\left[u_{i}\right]=-$ Dispersion $-\left(1-2 \omega \tau+\omega \tau^{2}\right) \cdot$ Volatility $-\omega(1-\tau)^{2} \cdot$ Variance of $\phi$-Information cost.

The above expressions for expected payoff are relevant to P-NE. As far as N-NE are concerned, setting $\beta_{i}=\beta=0$ in (D.28) yields:

$$
E\left[u_{i}\right]_{\beta=0}=-\frac{\left(1-2 \omega \tau+\omega \tau^{2}\right)}{\alpha}-\frac{\omega(1-\tau)^{2}}{\gamma}
$$

where the terms correspond to:

$$
E\left[u_{i}\right]_{\beta=0}=-\left(1-2 \omega \tau+\omega \tau^{2}\right) \cdot \text { Volatility }-\omega(1-\tau)^{2} \cdot \text { Variance of } \phi .
$$

\section{D.3 Characterising the solution to the CB's min- imisation problem}

Under the assumption of costly private information, the CB aims to minimise the expected loss function given by (4.38):

$$
E[L]_{\beta=\beta^{*}}=\frac{\alpha \tau^{2} p}{(1-\omega \tau)^{2}}+(1-\tau)^{2} \cdot \frac{1}{\gamma}
$$

\footnotetext{
${ }^{1}$ The assumed form of the linear solution for $a_{i}$ implies that the weights in (4.5) and (4.13) add up to unity, which eliminates $\theta$ from the payoff expression. This ensures that the expected payoff is defined, as the variance of $\theta$ is not defined.
} 
The first-order condition for minimising expected loss in (4.38) with respect to $\tau$ is quartic in $\tau$ :

$$
\frac{\partial E[L]_{\beta=\beta^{*}}}{\partial \tau}=\frac{2 \alpha \tau p}{(1-\omega \tau)^{3}}-\frac{2(1-\tau)}{\gamma}=0 .
$$

Solving the first-order condition yields four intractable roots. Numerical examples suggest that two roots are complex and one root is above unity, leaving only one admissible root. In what follows we show, in general terms, that there exists a unique root that lies between zero and unity.

Firstly, CB's expected loss is continuous in $\tau$ if $\omega \tau<1$. Secondly, the first derivative of $E[L]_{\beta=\beta^{*}}$ with respect to $\tau$ is negative when evaluated at $\tau=0$ and positive when evaluated at $\tau=1$. Thus, as shown in Figure D.1, expected loss is falling in the vicinity of $\tau=0$ and rising in the vicinity of $\tau=1$.

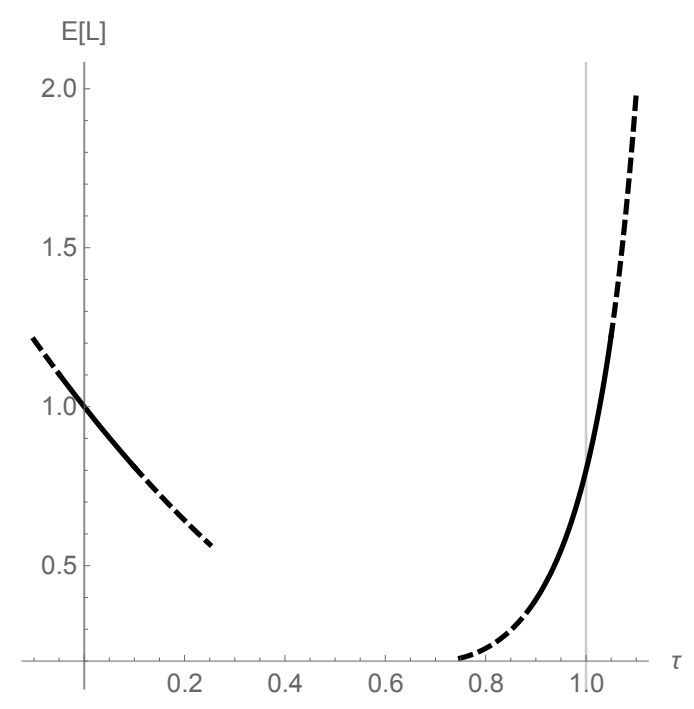

Figure D.1: Private information acquisition. The CB's expected loss in the vicinity of $\tau=0$ and $\tau=1$.

This means that the derivative changes its sign from negative to positive at least once over the interval $\tau \in(0,1)$, meaning that there exists at least one interior minimum. In order to show that the interior minimum is unique, we look at the conditions under which the sign of the first derivative of $E[L]_{\beta=\beta^{*}}$ with respect to $\tau$ is negative or positive. The sign of the derivative is negative for $0<\gamma<\gamma_{\text {crit }}$ and positive for $\gamma_{\text {crit }}<\gamma$, where:

$$
\gamma_{\text {crit }} \equiv \frac{(1-\tau)(1-\omega \tau)^{3}}{\alpha \tau p}
$$

Critical value for $\gamma$ in (D.31) is monotonically decreasing in $\tau$. In the limit, as $\tau$ approaches zero, $\gamma_{\text {crit }}$ tends to infinity; and in the limit, as $\tau$ approaches unity, $\gamma_{\text {crit }}$ 
is equal to zero. Thus, as demonstrated in Figure D.2, actual $\gamma$ is equal to $\gamma_{\text {crit }}$ for a unique value of $\tau$. At this value of $\tau$, the first derivative of $E[L]_{\beta=\beta^{*}}$ with respect to $\tau$ changes its sign from negative to positive, hence, this value is the unique solution $\tau=\tau^{P}$.

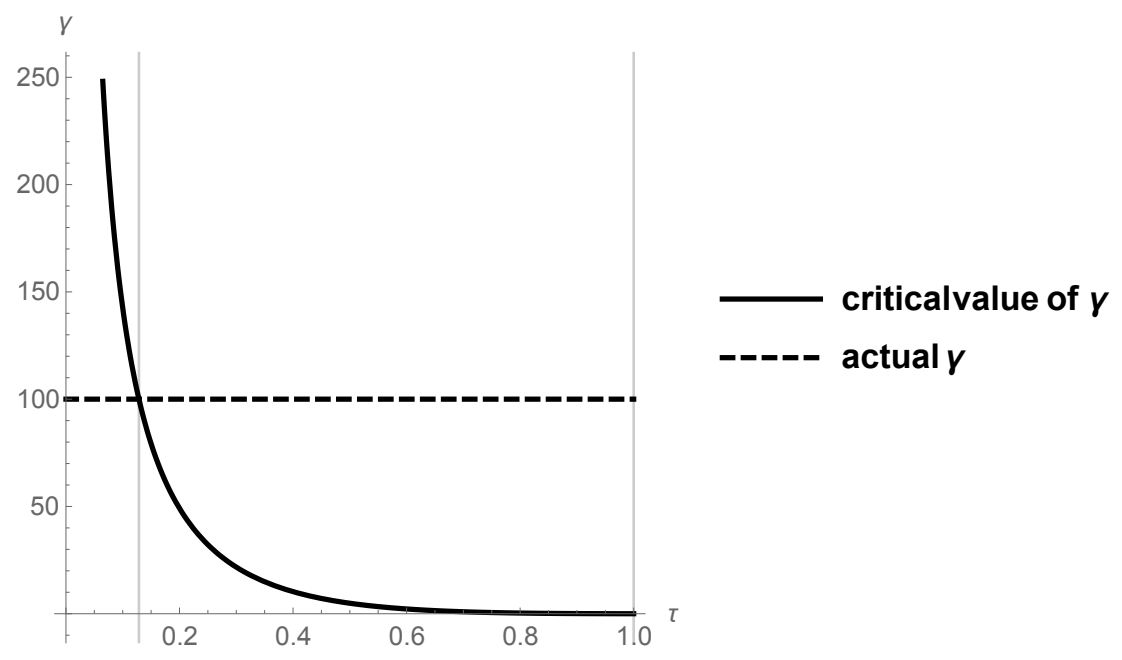

Figure D.2: Private information acquisition. Critical and actual values of $\gamma$ as a function of $\tau$.

The above argument for the existence of a unique interior minimum equally applies to the problem of minimising CB's expected loss, when the latter is expressed as in (4.37):

$$
E[L]_{\kappa=\kappa^{*}}=\frac{\alpha \tau^{2}}{[\alpha+\beta(1-\omega \tau)]^{2}}+(1-\tau)^{2} \cdot \frac{1}{\gamma},
$$

where $\beta$ is assumed to be fixed. This is of relevance in Section 4.3.4.1, in which we discuss public information acquisition. 


\section{Bibliography}

Adam, K. (2007). Optimal monetary policy with imperfect common knowledge. Journal of Monetary Economics, 54(2):267-301.

Amador, M. and Weill, P.-O. (2010). Learning from prices: Public communication and welfare. Journal of Political Economy, 118(5):866-907.

Angeletos, G.-M. and Pavan, A. (2007a). Efficient use of information and social value of information. Econometrica, 75(4):1103-1142.

Angeletos, G.-M. and Pavan, A. (2007b). Socially optimal coordination: Characterization and policy implications. Journal of the European Economic Association, 5(2-3):585-593.

Arato, H., Hori, T., and Nakamura, T. (2014). Endogenous information acquisition and the partial announcement policy. Discussion Paper 0892, ISER.

Azariadis, C. (1981). Self-fulfilling prophecies. Journal of Economic Theory, 25(3):380-396.

Barro, R. J. and Gordon, D. B. (1983). A positive theory of monetary policy in a natural rate model. Journal of Political Economy, 91(4):589 - 610.

Batini, N. and Nelson, E. (2001). The lag from monetary policy actions to inflation: Friedman revisited. International Finance, 4(3):381-400.

Begg, D. K. H. and Imperato, I. (2001). The rationality of information gathering: Monopoly. The Manchester School, 69(3):237-252.

Bernanke, B. S. and Woodford, M. (1997). Inflation forecasts and monetary policy. Journal of Money, Credit and Banking, 29(4):653-684.

Bond, P. and Goldstein, I. (2015). Government intervention and information aggregation by prices. The Journal of Finance, 70(6):2777-2812.

Bulow, J. I., Geanakoplos, J. D., and Klemperer, P. D. (1985). Multimarket oligopoly: Strategic substitutes and complements. The Journal of Political Economy, 93(3):488-511. 
Campbell, J., Evans, C., Fisher, J., and Justiniano, A. (2012). Macroeconomic effects of fomc forward guidance. Brookings Papers on Economic Activity, 43.

Carlsson, H. and van Damme, E. (1993). Global games and equilibrium selection. Econometrica: Journal of the Econometric Society, 61(5):989-1018.

Cass, D. and Shell, K. (1983). Do sunspots matter? The Journal of Political Economy, 92(2):193-227.

Colombo, L. and Femminis, G. (2008). The social value of public information with costly information acquisition. Economics Letters, 100(2):196-199.

Colombo, L., Femminis, G., and Pavan, A. (2014). Information acquisition and welfare. The Review of Economic Studies, 81:1438-1483.

Cooper, R. and John, A. (1988). Coordinating coordination failures in keynesian models. The Quarterly Journal of Economics, 103(3):441-463.

Cornand, C. and Heinemann, F. (2008). Optimal degree of public information dissemination*. The Economic Journal, 118(528):718-742.

Cukierman, A. (2009). The limits of transparency. Economic Notes, 38(1-2):1-37.

Cukierman, A. and Meltzer, A. H. (1986). The credibility of monetary announcements. In Neumann, M. J. M., editor, Monetary Policy and Uncertainty, pages 39-68. Baden-Baden: Nomos Verlagsgesellschaft.

D'Agostino, A. and Whelan, K. (2008). Federal Reserve information during the great moderation. Journal of the European Economic Association, 6(2-3):609-620.

Faust, J. and Svensson, L. E. (2001). Transparency and credibility: monetary policy with unobservable goals. International Economic Review, 42(2):369-397.

Fry, M., Julius, D., Mahadeva, L., Roger, S., and Sterne, G. (2000). Key issues in the choice of monetary policy framework. In Mahadeva, L. and Sterne, G., editors, Monetary Policy Frameworks in a Global Context, pages 1-216. London: Routledge, London.

Fuhrer, J. C. (1994). Goals, guidelines, and constraints facing monetary policymakers: an overview. New England Economic Review, pages 3-16.

Gabaix, X. and Laibson, D. (2002). The 6d bias and the equity-premium puzzle. In NBER Macroeconomics Annual 2001, Volume 16, pages 257-330. MIT Press. 
Gärtner, M. and Griesbach, B. (2012). Rating agencies, self-fulfilling prophecy and multiple equilibria?: An empirical model of the European sovereign debt crisis 2009-2011. School of Economics and Political Science, Department of Economics, University of St. Gallen.

Geraats, P. M. (2002). Central bank transparency*. The Economic Journal, 112(483):F532-F565.

Goodfriend, M. (1986). Monetary mystique: Secrecy and central banking. Journal of Monetary Economics, 17(1):63-92.

Hahn, V. (2010). Transparency in monetary policy, signaling, and heterogeneous information. Working paper, Center of Economic Research at ETH Zurich.

Hahn, V. (2012). Should central banks remain silent about their private information on cost-push shocks? Oxford Economic Papers, 64(4):593-615.

Harsanyi, J. and Selten, R. (1988). A General Theory of Equilibrium Selection in Games. Cambridge, MA: Massachusetts Institute of Technology Press.

Hayek, F. A. (1945). The use of knowledge in society. The American economic review, 35(4):519-530.

Hellwig, C. (2005). Heterogeneous information and the welfare effects of public information disclosures. unpublished, UCLA.

Hellwig, C. and Veldkamp, L. (2009). Knowing what others know: Coordination motives in information acquisition. The Review of Economic Studies, 76(1):223251.

Issing, O. (2005). Communication, transparency, accountability: monetary policy in the twenty-first century. Federal Reserve Bank of St. Louis Review, 87(2):65-83.

James, J. G. and Lawler, P. (2011). Optimal policy intervention and the social value of public information. The American Economic Review, 101(4):1561-1574.

James, J. G. and Lawler, P. (2012a). Heterogeneous information quality; strategic complementarities and optimal policy design. Journal of Economic Behavior \&6 Organization, 83(3):342-352.

James, J. G. and Lawler, P. (2012b). Strategic complementarity, stabilization policy, and the optimal degree of publicity. Journal of Money, Credit and Banking, 44(4):551-572. 
Keynes, J. M. (1936). General theory of employment, interest and money. London: Macmillan.

Knight, F. H. (1921). Risk, uncertainty and profit. New York: Hart, Schaffner and Marx.

Kydland, F. E. and Prescott, E. C. (1977). Rules rather than discretion: The inconsistency of optimal plans. The Journal of Political Economy, 85(3):473-491.

Lubik, T., Matthes, C., and Mertens, E. (2020). Indeterminacy and imperfect information.

Mankiw, N. G. (1985). Small menu costs and large business cycles: A macroeconomic model of monopoly. The Quarterly Journal of Economics, 100(2):pp. 529-537.

Mankiw, N. G. and Reis, R. (2002). Sticky information versus sticky prices: a proposal to replace the New Keynesian Phillips curve. The Quarterly Journal of Economics, 117(4):1295-1328.

Mankiw, N. G. and Reis, R. (2003). Sticky information: A model of monetary nonneutrality and structural slumps. In Aghion, P., Frydman, R., Stiglitz, J., and Woodford, M., editors, Knowledge, Information, and Expectations in Modern Macroeconomics: In Honor of Edmund S. Phelps, pages 64-86. Princeton University Press.

Mankiw, N. G. and Reis, R. (2006). Pervasive stickiness (expanded version). Technical report, National Bureau of Economic Research.

McCallum, B. T. (1993). Discretion versus policy rules in practice: two critical points: A comment. In Carnegie-Rochester Conference Series on Public Policy, volume 39, pages 215-220. Elsevier.

McCallum, B. T. (1997). Issues in the design of monetary policy rules. Technical report, National Bureau of Economic Research.

McCallum, B. T. (2005). Michael Woodford's interest and prices - a review article. Carnegie Mellon University.

Merton, R. K. (1948). The social psychology of housing. In Dennis, W., editor, Current Trends in Social Psychology, pages 163-217. Pittsburgh: University of Pittsburgh Press.

Mishkin, F. S. (2004). Can central bank transparency go too far? Technical report, National Bureau of Economic Research. 
Morris, S. (2008). Global games. In Durlauf, S. N. and Blume, L. E., editors, The New Palgrave Dictionary of Economics, volume 3, pages 676-681. Palgrave Macmillan, 2nd edition.

Morris, S. and Shin, H. S. (1998). Unique equilibrium in a model of self-fulfilling currency attacks. American Economic Review, pages 587-597.

Morris, S. and Shin, H. S. (2000). Rethinking multiple equilibria in macroeconomic modeling. NBER macroeconomics Annual, 15:139-161.

Morris, S. and Shin, H. S. (2002). Social value of public information. The American Economic Review, 92(5):1521-1534.

Morris, S. and Shin, H. S. (2005). Central bank transparency and the signal value of prices. Brookings Papers on Economic Activity, 2005(2):1-66.

Morris, S. and Shin, H. S. (2008). Coordinating expectations in monetary policy. In Touffut, J. P., editor, Central Banks as Economic Institutions, pages 88-104. Cheltenham: Edward Elgar Publishing.

Morris, S. and Shin, H. S. (2018). Central bank forward guidance and the signal value of market prices. In AEA Papers and Proceedings, volume 108, pages 572-77.

Morris, S., Shin, H. S., and Tong, H. (2006). Social value of public information: Morris and shin (2002) is actually pro-transparency, not con: Reply. The American Economic Review, 96(1):453-455.

Myatt, D. P. and Wallace, C. (2012). Endogenous information acquisition in coordination games. The Review of Economic Studies, 79(1):340-374.

Reis, R. (2004). Inattentive consumers. Technical report, National Bureau of Economic Research.

Reis, R. (2006a). Inattentive consumers. Journal of monetary Economics, 53(8):1761-1800.

Reis, R. (2006b). Inattentive producers. The Review of Economic Studies, 73(3):793821.

Rogoff, K. (1985). The optimal degree of commitment to an intermediate monetary target. The Quarterly Journal of Economics, 100(4):1169-1189.

Romer, C. D. and Romer, D. H. (2000). Federal reserve information and the behavior of interest rates. The American Economic Review, 90(3):429-457. 
Sims, C. A. (1998). Stickiness. In Carnegie-Rochester Conference Series on Public Policy, volume 49, pages 317-356. Elsevier.

Svensson, L. E. (1999). Inflation targeting as a monetary policy rule. Journal of monetary economics, 43(3):607-654.

Svensson, L. E. O. (1997). Inflation forecast targeting: Implementing and monitoring inflation targets. European Economic Review, 41(6):1111-1146.

Svensson, L. E. O. (2006). Social value of public information: Comment: Morris and shin (2002) is actually pro-transparency, not con. The American Economic Review, 96(1):448-452.

Taylor, J. B. (1993). Discretion versus policy rules in practice. In Carnegie-Rochester conference series on public policy, volume 39, pages 195-214. Elsevier.

Valukas, A. R. (2010). Lehman brothers holdings inc. chapter 11 proceedings examiner's report. Technical report, US Bankruptcy Court Southern District of New York, Jenner and Block LLP.

Von Hippel, E. (1994). "Sticky information" and the locus of problem solving: implications for innovation. Management science, 40(4):429-439.

Von Stackelberg, H. (2010). Market structure and equilibrium. Springer Science \& Business Media.

Walsh, C. E. (1995). Optimal contracts for central bankers. American Economic Review, 85(1):150-167.

Walsh, C. E. (2010). Monetary theory and policy. MIT press.

Woodford, M. (1994). Nonstandard indicators for monetary policy: can their usefulness be judged from forecasting regressions? In Monetary Policy, pages 95-115. The University of Chicago Press.

Woodford, M. (1999). Commentary: How should monetary policy be conducted in an era of price stability? In New Challenges for Monetary Policy: A Symposium Sponsored by the Federal Reserve Bank of Kansas City, pages 277-316. Federal Reserve Bank of Kansas City.

Woodford, M. (2002). Inflation stabilization and welfare. The BE Journal of Macroeconomics, 2(1).

Zbaracki, M., Ritson, M., Levy, D., Dutta, S., and Bergen, M. (2000). The managerial and customer dimensions of the cost of price adjustment: Direct evidence from industrial markets. Unpublished manuscript. 